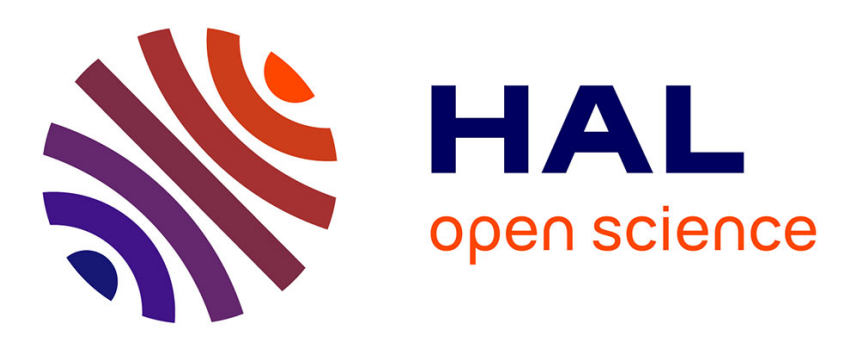

\title{
The Stability and Reactivity of Activated Acryloylcarbamates as Reagents for the Synthesis of N-1 Substituted Thymine and Uracil - An NMR and DFT Study
}

Dominik Rejman, Radek Pohl, Lubomír Rulíšek

\section{To cite this version:}

Dominik Rejman, Radek Pohl, Lubomír Rulíšek. The Stability and Reactivity of Activated Acryloylcarbamates as Reagents for the Synthesis of N-1 Substituted Thymine and Uracil - An NMR and DFT Study. Journal of Physical Organic Chemistry, 2010, 24 (5), pp.423. 10.1002/poc.1775 . hal-00599797

\section{HAL Id: hal-00599797 \\ https://hal.science/hal-00599797}

Submitted on 11 Jun 2011

HAL is a multi-disciplinary open access archive for the deposit and dissemination of scientific research documents, whether they are published or not. The documents may come from teaching and research institutions in France or abroad, or from public or private research centers.
L'archive ouverte pluridisciplinaire HAL, est destinée au dépôt et à la diffusion de documents scientifiques de niveau recherche, publiés ou non, émanant des établissements d'enseignement et de recherche français ou étrangers, des laboratoires publics ou privés. 


\section{Journal of Physical Organic Chemistry}

WILEY

\section{The Stability and Reactivity of Activated Acryloylcarbamates as Reagents for the Synthesis of N-1 Substituted Thymine and Uracil - An NMR and DFT Study}

\begin{tabular}{|c|c|}
\hline Journal: & Journal of Physical Organic Chemistry \\
\hline Manuscript ID: & POC-10-0018.R1 \\
\hline Wiley - Manuscript type: & Research Article \\
\hline $\begin{array}{r}\text { Date Submitted by the } \\
\text { Author: }\end{array}$ & 14-Apr-2010 \\
\hline Complete List of Authors: & $\begin{array}{l}\text { Rejman, Dominik; Institute of Organic Chemistry and Biochemistry } \\
\text { CAS v.v.i., Medicinal Chemistry II } \\
\text { Pohl, Radek; Institute of Organic Chemistry and Biochemistry CAS } \\
\text { v.v.i., Dept. of NMR } \\
\text { Rulíš́ek, Lubomír; Institute of Organic Chemistry and Biochemistry } \\
\text { CAS v.v.i., Computational Chemistry }\end{array}$ \\
\hline Keywords: & uracil, thymine, mechanism, NMR, DFT \\
\hline
\end{tabular}

\section{s ScholarONE" \\ Manuscript Central}


Figure 1. The planar geometries of E-7b investigated by density functional theory calculations. 


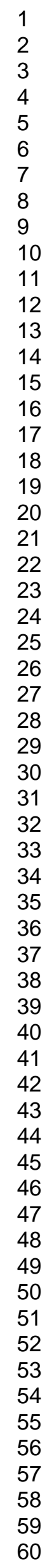

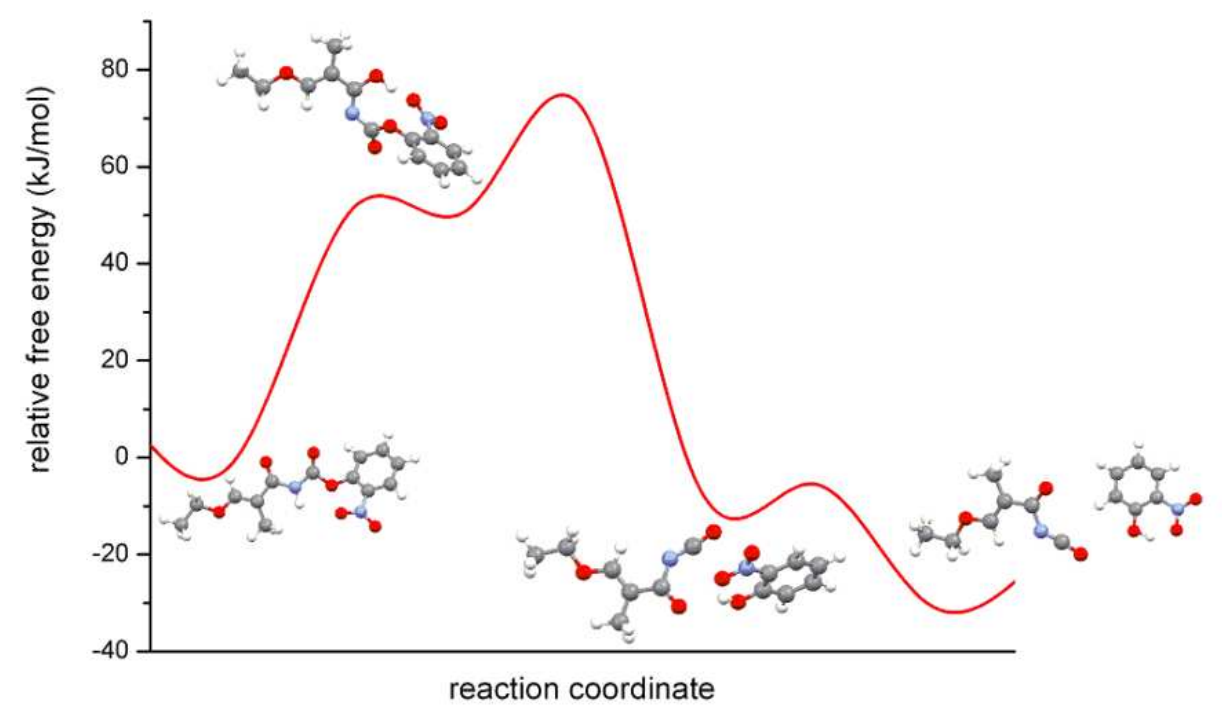

Figure 2. The reaction coordinate for the degradation of $\mathrm{E}-7 \mathrm{~b}$. $80 \times 51 \mathrm{~mm}$ (300 x 300 DPI) 

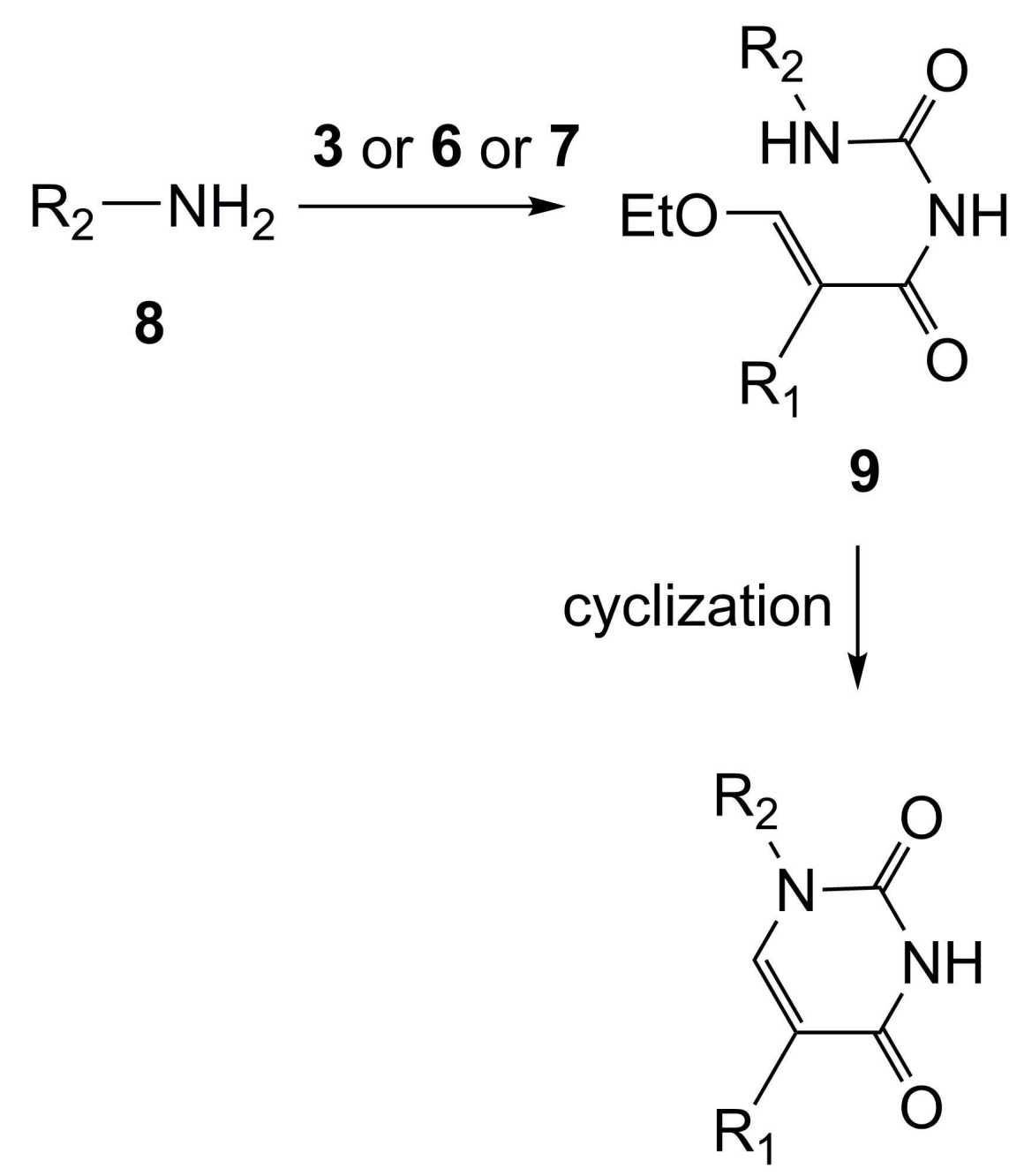

$$
\begin{aligned}
& \mathrm{R}_{1}=\mathrm{H}, \mathrm{CH}_{3} \\
& \mathrm{R}_{2}=\text { alkyl, cycloalkyl, aryl, hetaryl }
\end{aligned}
$$
Scheme 2. The reaction of reagents 3,6 and 7 with amines and subsequent cyclization providing 1 -
N-substituted pyrimidines.
$71 \times 98 \mathrm{~mm}(600 \times 600 \mathrm{DPI})$ 

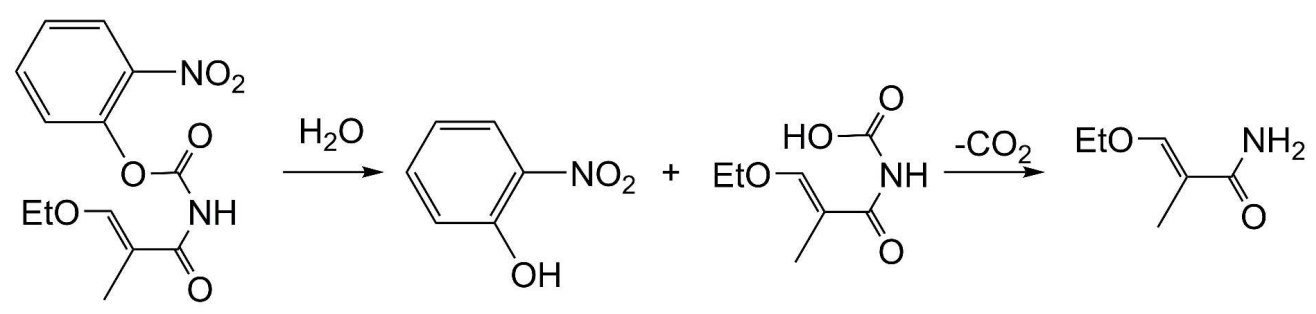

$7 b$

Scheme 3. The decomposition of 7b via hydrolysis and decarboxylation. $159 \times 43 \mathrm{~mm}(600 \times 600 \mathrm{DPI})$ 
1

2

3

4

5

6

7

8

9

10

11

12

13

14

15

16

17

18

19

20

21

22

23

24

25

26

27

28

29

30

31

32

33

34

35

36

37

38

39

40

41

42

43

44

45

46

47

48

49

50

51

52

53

54

55

56

57

58

59

60

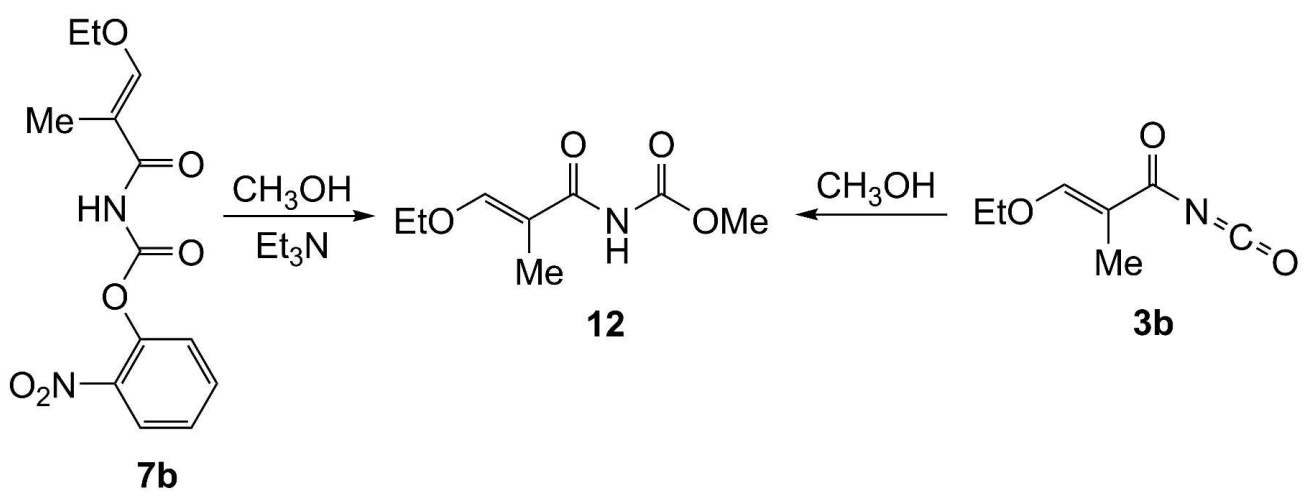

Scheme 4 . The chemical proof of $3 \mathrm{~b}$ structure. $153 \times 58 \mathrm{~mm}(600 \times 600 \mathrm{DPI})$ 


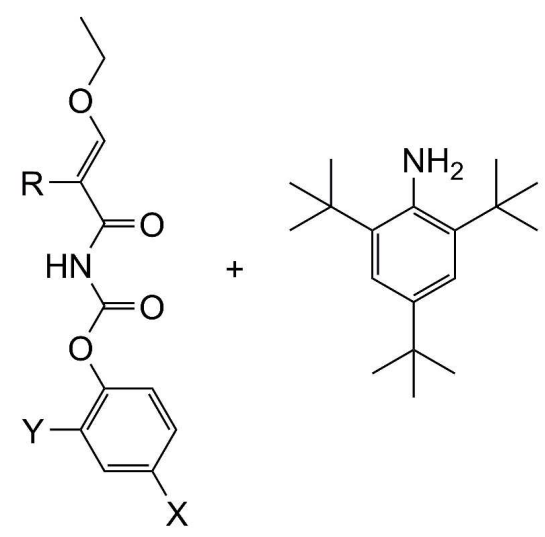

7a: $\mathrm{R}=\mathrm{H}, \mathrm{X}=\mathrm{H}, \mathrm{Y}=\mathrm{NO}_{2}$

7b: $\mathrm{R}=\mathrm{CH}_{3}, \mathrm{X}=\mathrm{H}, \mathrm{Y}=\mathrm{NO}_{2}$

7c: $\mathrm{R}=\mathrm{H}, \mathrm{X}=\mathrm{NO}_{2}, \mathrm{Y}=\mathrm{H}$

7d: $\mathrm{R}=\mathrm{CH}_{3}, \mathrm{X}=\mathrm{NO}_{2}, \mathrm{Y}=\mathrm{H}$

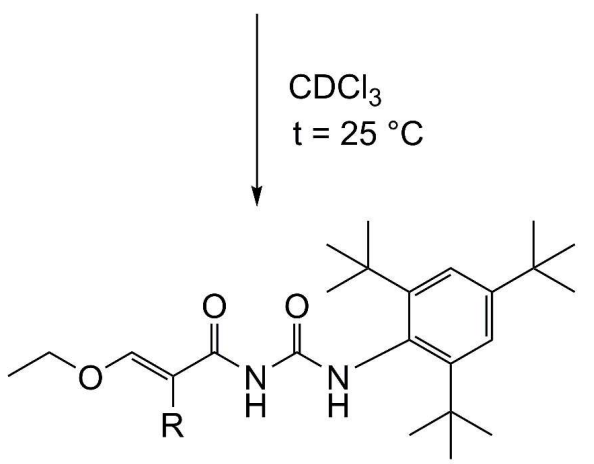

11a: $\mathrm{R}=\mathrm{H}$

11b: $\mathrm{R}=\mathrm{CH}_{3}$

$+$<smiles>[X]c1ccc(O)c([Y])c1</smiles>

Scheme 6 . The reaction of 7a-d with 2,4,6-tri-tert-butylaniline.

$72 \times 196 \mathrm{~mm}(600 \times 600 \mathrm{DPI})$ 
The mechanism of the decomposition of acryloylcarbamates yielding highly reactive isocyanates was proposed based on NMR measurements and quantum chemical calculations. Their reactivity with 2,4,6-tri-tert-butylaniline showed their usefulness in synthesis of $N-1$ substituted thymines and uracils.

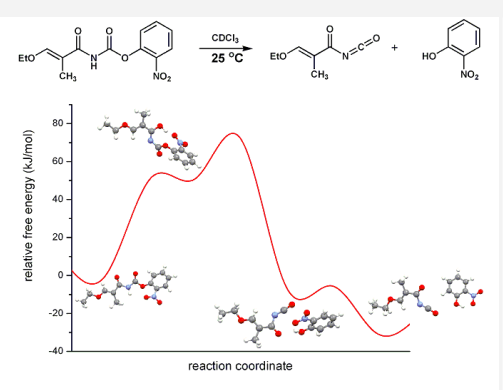

The Stability and Reactivity of Activated

Acryloylcarbamates as Reagents for the

Synthesis of $N-1$ Substituted Thymine and Uracil - A NMR and DFT Study

Radek Pohl, ${ }^{*}$ Lubomír Rulíšek, ${ }^{*}$ and Dominik Rejman* 
<smiles>[R]/C(=C\OCC)C(=O)OCC</smiles>

1: $\mathrm{R}=\mathrm{H}, \mathrm{CH}_{3}$

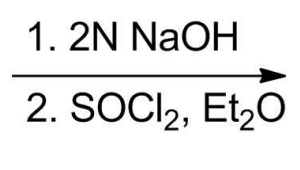<smiles>[R]/C(=C\OCC)C(=O)Cl</smiles>

2: $\mathrm{R}=\mathrm{H}, \mathrm{CH}_{3}$<smiles>[R]C=COC[CH2+]C(C)N(CC)CCOC=C([R7])C(=O)N=C=O</smiles>

4a: $\mathrm{R}=\mathrm{H}$

5

3a: $\mathrm{R}=\mathrm{H}$

4b: $\mathrm{R}=\mathrm{CH}_{3}$

3b: $\mathrm{R}=\mathrm{CH}_{3}$

6a: $\mathrm{R}=\mathrm{H}$

6b: $\mathrm{R}=\mathrm{CH}_{3}$

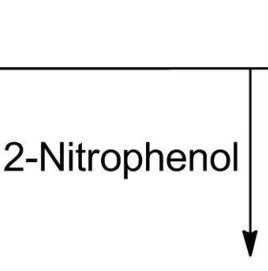<smiles>[R]/C=C(/OCC)C(=O)NC(=O)Oc1ccccc1[N+](=O)[O-]</smiles>

7a: $\mathrm{R}=\mathrm{H}$

7b: $\mathrm{R}=\mathrm{CH}_{3}$
4-Nitrophenol<smiles>[R]/C(=C\OCC)C(=O)NC(=O)Oc1ccc([N+](=O)[O-])cc1</smiles>

7c: $\mathrm{R}=\mathrm{H}$

7d: $\mathrm{R}=\mathrm{CH}_{3}$ 


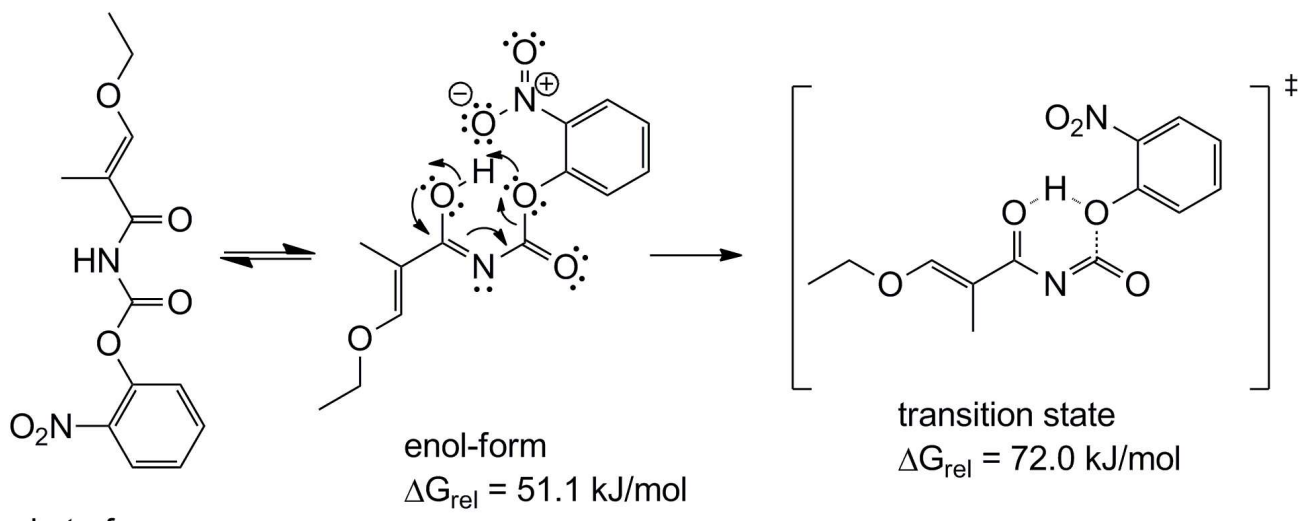

keto-form $\Delta \mathrm{G}_{\mathrm{rel}}=0 \mathrm{~kJ} / \mathrm{mol}$

products $\Sigma \Delta \mathrm{G}_{\text {rel }}=-30.2 \mathrm{~kJ} / \mathrm{mol}$

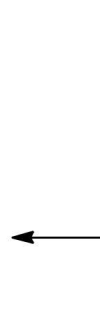<smiles>CCOC=C(C)C(=O)N=C=O</smiles>

product complex $\Delta \mathrm{G}_{\mathrm{rel}}=-5.6 \mathrm{~kJ} / \mathrm{mol}$

$163 \times 135 \mathrm{~mm}(300 \times 300 \mathrm{DPI})$ 


\title{
The Stability and Reactivity of Activated Acryloylcarbamates as Reagents \\ for the Synthesis of $N-1$ Substituted Thymine and Uracil - An NMR and DFT Study
}

\author{
Radek Pohl, ${ }^{*[a]}$ Lubomír Rulíšek, ${ }^{*[a]}$ and Dominik Rejman*a] \\ [a] Institute of Organic Chemistry and Biochemistry AS CR, v.v.i., Flemingovo nám. 2, 16610 Prague 6, Czech Republic \\ Fax: +420 220183123 \\ E-mail: pohl@uochb.cas.cz; rulisek@uochb.cas.cz; rejman@uochb.cas.cz
}

Keywords: uracil / thymine / nucleosidation / mechanism / NMR / DFT

\begin{abstract}
:
The mechanism of the decomposition of acryloylcarbamates $\mathbf{7 a - b}$ yielding highly reactive isocyanates 3a-b was proposed based on NMR measurements and quantum chemical calculations. A good agreement between the experimental kinetic data and DFT calculations allowed us to demonstrate that the stability of 7a-d depends on the presence of methyl in the acryloyl moiety and the position of nitro group in the nitrophenolic part of the molecule. Furthermore, the reactivity of 7a-d with weakly nucleophilic and sterically hindered 2,4,6-tritert-butylaniline was explored by ${ }^{1} \mathrm{H}$ NMR demonstrating the usefulness of reagents $7 \mathbf{a}-\mathbf{d}$ offering access to a variety of $1-N$-substituted uracils and thymines with potentially interesting biological properties.
\end{abstract}

\section{Introduction}

Many biologically active compounds belong to the class of nucleosides and nucleotides analogues, which play an important role in anticancer therapy ${ }^{1-2}$ or act as antiviral compounds. ${ }^{3}$ Specific examples also involve carbocyclic nucleosides ${ }^{4-5}$ (Carbovir®) and acyclic phosphonate nucleotides ${ }^{6}$ (Viread, Cidofovir, Tenofovir, Hepsera), which represent biologically important compounds in which the nucleobase is not connected via a glycosidic bond.

From a synthetic point of view, a crucial step in their preparation is the attachment of the nucleobase - the nucleosidation reaction. The most common synthetic routes to purine 
nucleoside analogues are represented by direct alkylation of the appropriate nucleobases (adenine, 6-chloropurine or 2-amino-6-chloropurine) with the halo, tosyloxy or mesyloxy derivatives in dipolar aprotic solvents (DMF, DMSO) using cesium carbonate as a base. ${ }^{7-9}$ Alternatively, Mitsunobu reaction of 6-chloropurine or 2-amino-6-chloropurine with appropriate hydroxyderivative can be used. ${ }^{8,10-11}$ Recently, $N^{6}$-bis-Boc-protected adenine was successfully used as a substrate for the Mitsunobu reaction during the synthesis of neplanocin A. ${ }^{12}$ However, all of these methods for the introduction of pyrimidine nucleobases (uracil, thymine, and cytosine) suffer from both low regioselectivity and low yield of the 1-Nsubstituted product. The direct alkylation of nucleobases is usually accompanied by a competing elimination reaction, which further decreases the yield of the desired product. ${ }^{8-9}$ Some improvement (both in regioselectivity and overall yield) can be obtained through the use of 2,4-dimethoxypyrimidine or 2,4-dimethoxy-6-methylpyrimidine for the alkylation instead of uracil or thymine, respectively. ${ }^{13-15}$ As an alternative approach to the pyrimidine nucleoside analogues (e.g. carbocyclic nucleosides), a synthesis of 1-N-substituted nucleobases starting from a primary amine and isocyanates $\mathbf{3 a - b}$ or carbamates $\mathbf{6 a - b}$ were described fifty years ago by Shaw and Warrener. ${ }^{16}$ The key isocyanates 3a-b, accessible through two independent routes ${ }^{17-18}$ (Scheme 1), were used as intermediates for the synthesis of carbamates 6a-b described by Hřebabecký et al. ${ }^{19}$

Scheme 1. The reagents for the preparation of $1-N$-substituted pyrimidine nucleobases.

Isocyanates 3a-b are an unusually moisture-sensitive species and also very reactive compounds, capable of forming carbamates with free hydroxy groups. ${ }^{20}$ Therefore, the reaction is usually carried out either with the appropriately protected substrate or without protection at low temperature $\left(<-20^{\circ} \mathrm{C}\right)$. This property seems to be the main factor responsible for the varying yield of the reported uracil and thymine derivatives (usually $\sim 20$ $80 \%$, in most cases $<60 \%$ ) found in the literature. ${ }^{21-32}$ The formation of uracil and thymine rings is a two-stage reaction consisting of the aminolysis of carbamates $\mathbf{6 a - b}$ or the addition of amine to isocyanates $\mathbf{3 a - b}$ followed by a cyclization of the acryloylureas $\mathbf{9}$ formed (Scheme 2). ${ }^{33}$

Scheme 2. The reaction of reagents $\mathbf{3 , 6}$ and $\mathbf{7}$ with amines and subsequent cyclization providing $1-N$-substituted pyrimidines. 
To date, there has not been any general, high-yield method available for the attachment of uracil and thymine moieties to sterically hindered secondary, tertiary, or even aromatic carbon atoms.

It was the extreme reactivity and moisture sensitivity of isocyanates $\mathbf{3}$ on the one hand and the low yields of $\mathbf{9}$ obtained using carbamates $\mathbf{6}$ on the other that prompted us to develop more advantageous reagents 7 , which proved to be reactive enough with a variety of amines. Moreover, they can be stored for longer time (several months in refridgerator), which enabled us to use them in modular syntheses of uracils and thymines $10^{34}$ The synthesis of the reagents $7 \mathbf{a}-\mathbf{d}$ followed the procedure described for $\mathbf{6},{ }^{19}$ in whose last step of the 'one-pot' synthesis, a 2-nitrophenol solution in dioxane was added instead of ethanol.

The reagents $\mathbf{7 a}-\mathbf{d}$ were tested in reactions with a wide variety of amines (including those bonded to secondary, tertiary, and aromatic systems), giving consistently high yields of corresponding 1,3-disubstituted urea derivatives. The desired uracil and thymine derivatives were obtained by the cyclization of the urea intermediates using Dowex 50 in $\mathrm{H}^{+}$form, providing again very high yields. ${ }^{34}$

In this work, we wish to address an unprecedented decomposition of the reagent $\mathbf{7 b}$ in $\mathrm{CDCl}_{3}$ solution observed in the ${ }^{1} \mathrm{H}$ NMR measurements, to analyze and quantitatively characterize the reaction mechanism of the decomposition reaction. To this end, we conducted a series of NMR experiments to yield reliable kinetic data and a series of DFT calculations to provide us with the mechanistic view of the studied reaction. In order to understand the reaction mechanism in deatail, we have compared the experimental data with other reagents $7 \mathbf{a}, 7 \mathbf{c}$ and 7d. We have also investigated reaction of 7a-d with low nucleophilic and sterically hindered 2,4,6-tri-tert-butylaniline to ensure that decomposition of $\mathbf{7 a - b}$ does not limit their synthetic usage in preparation of $N-1$ substituted thymines and uracils. These findings represent a useful and successful conjunction of theoretical and experimental efforts extending our understanding of the organic reactions pertinent to this class of compounds.

\section{Results and Discussion}

\section{Kinetics of decomposition studied by NMR}

Reagents 7a-d were prepared according Scheme 1 and fully characterized by ${ }^{1} \mathrm{H}$ and ${ }^{13} \mathrm{C}$ NMR, elemental analysis, IR and MS. In the course of the NMR characterization of $\mathbf{7 b}$, we found that the reagent decomposes, yielding a complex mixture. The ${ }^{1} \mathrm{H}$ NMR spectrum of $\mathbf{7 b}$ 
Scheme 3. The decomposition of $\mathbf{7 b}$ via hydrolysis and decarboxylation.

To rule out the effect of water, which might be present in regular $\mathrm{CDCl}_{3}$, the ${ }^{1} \mathrm{H}$ NMR spectra were recorded in dried $\mathrm{CDCl}_{3}$ and a sealed NMR tube (see Experimental). The experiment has again shown the decomposition of compound $\mathbf{7 b}$, and the final products of decomposition were identified as acryloylisocyanate $\mathbf{3 b}$ and 2-nitrophenol. Reagent $\mathbf{7 b}$ was characterised by NMR only when a high concentration of sample and a short measurement time were applied.

To ascertain that acryloylisocyanate $\mathbf{3 b}$ is the decomposition product, we allowed the decomposed mixture to react with dry methanol, yielding methyl ester 12, i.e. the same product as that formed through the reaction of $\mathbf{7 b}$ with methanol in the presence of $\mathrm{Et}_{3} \mathrm{~N}$ (Scheme 4). In addition, isocyanate $\mathbf{3 b}$ was identified by IR measured in dry $\mathrm{CHCl}_{3}$ (the characteristic band being at $2244 \mathrm{~cm}^{-1}$ for asymmetric stretching vibrations of the -NCO group) and by ${ }^{13} \mathrm{C}$ NMR (with the characteristic chemical shift of -NCO carbon at 130.47 ppm).

Scheme 4. The chemical proof of $\mathbf{3 b}$ structure.

The rate of decomposition was estimated based on the ${ }^{1} \mathrm{H}$ NMR kinetic measurement in dry $\mathrm{CDCl}_{3}$ and sealed NMR tube at $25{ }^{\circ} \mathrm{C}$. The concentrations of starting compounds and decomposition product(s) were obtained by integration of several corresponding signals with similar relaxation times. The rate constant for the irreversible first order kinetic model was obtained from plots of the logarithm of the starting compound concentrations versus time, while rate constant for the reversible first order kinetic model was obtained from plots of the logarithm of the starting compound concentrations minus equilibrium concentration versus time and from equilibrium constant. Experimental data for decomposition of $\mathbf{7 b}$ fitted irreversible reaction with first order kinetics (Table 1 and supporting material) with half life $\left(t_{1 / 2}\right)$ of about 18 minutes. The kinetics measurements were also performed for the other 
reagents, $\mathbf{7 a}, \mathbf{7 c}$ and $\mathbf{7 d}$, and the results have been summarized in Table 1. The decomposition of 7a was reversible reaction with first order kinetics (Table 1 and supporting material), while compounds $\mathbf{7 c}$ and $\mathbf{7 d}$ were stable and did not decompose at all.

As arises from Table 1, the kinetic measurements demonstrate a surprisingly strong dependence on the reagents and their structure. Reagents $\mathbf{7 c}$ and $\mathbf{7 d}$ (possessing a nitro group in the para position) do not undergo any degradation. On the other hand, the derivatives with the ortho-nitro group in nitrophenol moiety decompose readily, with the decomposition of the 7b-bearing methyl group in the acryloyl part of the molecule being approximately fifty times faster than the decomposition of the 7a-lacking methyl substituent. The different stability of the reaction products reflected in the molecular structure was further explored by DFT calculations.

\section{Reaction mechanism of decomposition. Theoretical calculations.}

We first optimised all sixteen planar geometries for each of $\mathbf{7 a - d}$, viz. all the possible arrangements of four double or partial double bonds in the system (64 molecular geometries in total) at the DFT(PBE)/def2-SVP level with the single-point energy calculated at the DFT(B3LYP)/def2-TZVP level. The solvation effects and corrections to the ZPE energy, enthalpy and entropy (using the ideal gas approximation) were included as well (for details, see Experimental). The results of this extensive conformational search are summarised in Tables S1-S5 (see Supporting Info). As can be seen in Tables S1-S4, the calculations predict (in all cases) the most stable isomers to possess trans-configuration on the $-\mathrm{C}=\mathrm{C}$ - double bond. This fact has been corroborated experimentally by a missing NOE interaction between the Me group and the proton on the double bond for 7a--b or by a missing NOE between the protons of the double bond for 7c-d. At the same time we observe NOE between protons of $\mathrm{CH}_{2}$ group from $\mathrm{EtO}$ and $\mathrm{H}$ or $\mathrm{CH}_{3}$ on double bond in alpha position to $\mathrm{CO}$.

With regard to the proposed reaction mechanism, we have also investigated the possible isomers with the proton on either of the oxygens adjacent to the central - $\mathrm{NH}$ - group (these calculations were carried out only for the most stable isomers). The results are also listed in Tables S1-S4 (including various terms in overall Gibbs energies), and all twenty-four structures of $\mathbf{7 b}$ (assuming an $E$ configuration on the $-\mathrm{C}=\mathrm{C}$ - double bond) have been schematically depicted in Fig. 1. 
Figure 1. The planar geometries of $E-\mathbf{7 b}$ investigated by density functional theory calculations.

The calculations have shown that the $2 \mathrm{NH}-\mathrm{ZZZ}$ conformer is the most stable structure for the keto-tautomer of $\mathbf{7 b}$ whereas $3 \mathrm{OH}-\mathrm{ZZE}$ is the lowest energy enol-form of the same compound. It can be noted that $3 \mathrm{OH}-\mathrm{ZZE}$ geometry is nicely preorganised to form a six-membered transition state with bifurcated hydrogen bonding.

However, a rather surprising finding is the relatively small difference in energies between all the studied isomers. Considering the planar models depicted in Fig. 1, some of the structures would have been expected to have substantial intramolecular crowding. Even for such structures (e.g. 2NH-ZEE, 2NH-EEE) the energy or free energy differences amount to only $\sim 15-25 \mathrm{~kJ} \mathrm{~mol}^{-1}$. This demonstrates a relatively high level of flexibility in the studied molecules (the systems avoid crowding by adopting a helical form). Thus, we had to consider several reaction pathways for the elimination reaction. The lowest saddle points and their energies are summarised in Table 2 for all studied compounds (7a-7d) and for the proposed reaction mechanism of degradation shown in Scheme 5. It can be mentioned that all saddle points are characterized by a single imaginary frequency corresponding to the reactive coordinate and are therefore a true transition states for the studied reactions. By small changes of the geometry in the direction of reactant and product we ascertained that it connects the desired reactant and products structures (IRC-like calculation). A more complete listing of the calculated values of activation barriers can be found in Tables S1-S4.

Scheme 5 . The degradation of $\mathbf{7 b}-\mathbf{b}$ proposed mechanism.

Furthermore, the reaction coordinate with the structures of all the key points on the potential energy surface is depicted in Figure 2.

Figure 2. The reaction coordinate for the degradation of $E-\mathbf{7 b}$. 
As mentioned above, the transition state is characterised by the six-membered ring arrangement and is structurally close to the enol-form with activation barriers of 72-88 $\mathrm{kJ} \mathrm{mol}^{-1}$ (Table 2). We have also investigated an alternative pathway starting from the more stable keto-form and involving the direct hydrogen transfer from the nitrogen atom to the oxygen atom of the nitrophenol ring. The activation barrier involving a four-membered transition state was considerably higher ( 160-180 kJ.mol ${ }^{-1}$, see Tables S1, S3, S4) for the 2NH-EEZ isomer (which has the most favourable arrangement of the atoms and highest thermodynamic stability of the reactants). This clearly favours the pathways involving the sixmembered transition states described above.

As arises from Table 2, the presence of the ortho-nitro group plays an important role in the bifurcated hydrogen bond and lowers the free enthalpy of degradation $\Delta G_{\text {calc. On the other }}$ hand, the methyl group on the $-\mathrm{C}=\mathrm{C}$ - double bond lowers the free energy of activation, $\Delta G_{\text {calc }}^{\ddagger}$, likely due to the effect of hyperconjugation. The computational results are in good agreement with the experimentally calculated $\Delta G_{\text {exp }}^{\ddagger}$ computed from the rate constants using Eyring equation. Since the accurate ab initio determination of the absolute rate constant is beyond the scope of this work (for a thorough discussion on the subject, we refer the reader to Ref. 35), we mostly want to highlight the excellent agreement in the relative difference between the two reactions $(\mathbf{7 a}, \mathbf{7 b})$ for which the comparison is available. $\Delta \Delta G_{\exp }^{\ddagger}=9.7$ $\mathrm{kJ} . \mathrm{mol}^{-1}$, whereas $\Delta \Delta G_{\text {calc }}^{\ddagger}=11.9 \mathrm{~kJ} \cdot \mathrm{mol}^{-1}$.

\section{NMR characterisation of the reactivity of $7 \mathrm{a}-\mathrm{d}$ towards amines}

In our previous work, ${ }^{34}$ we showed that reagents $7 \mathbf{a}, \mathbf{b}$ react with a variety of amines, including acyclic, cyclic, and aromatic, and also amino acids. All of the reactions were completed within one hour of stirring at room temperature. Nevertheless, the reaction kinetics were not addressed in detail. Herein, the reactivity of 7a-d with low nucleophilic and sterically hindered 2,4,6-tri-tert-butylaniline is presented (Scheme 6).

Scheme 6. The reaction of 7a-d with 2,4,6-tri-tert-butylaniline.

The kinetic measurements were performed in dry $\mathrm{CDCl}_{3}$ at $25{ }^{\circ} \mathrm{C}$ and monitored by ${ }^{1} \mathrm{H} \mathrm{NMR}$. The concentrations of starting compounds and products were obtained by integration of 


\section{Conclusions}

It has been shown that the studied reagents $7 \mathbf{a}-\mathbf{d}$ are highly reactive even with lownucleophilic and sterically hindered amines and can be successfully applied for a modular synthesis of various $1-N$-substituted uracils and thymines, which is difficult to achieve with other synthetic methods. The stability of reagents $\mathbf{7 a - d}$ in dry $\mathrm{CDCl}_{3}$ was investigated by experimental and theoretical methods. It has been proved that the decomposition of the studied molecules is strongly affected by the presence of the Me-group on the $-\mathrm{C}=\mathrm{C}$ - double bond and the position of the nitro group on the phenol ring. While the former is assumed to lower the activation barrier of the reaction probably due to hyperconjugation, the latter plays a role in hydrogen bonding and influences the thermodynamics of the reaction. The experimental NMR observations are in good agreement with the results predicted by the DFT calculations, which allowed us to postulate a plausible reaction mechanism, presumably extending our understanding of the reactivity and stability of this class of compounds. It should be noted here that all the reagents are stable enough in the solid state to be stored for a long time. Moreover, the decomposition of the 2-nitrophenyl derivatives $\mathbf{7 a}$ and $\mathbf{7 b}$ yields an 
isocyanate product that could react in the same manner as the parent reagents. These compounds could be seen as an 'instant form' of the previously described isocyanates 3a-b.

\section{Experimental Section}

NMR Experiments: NMR spectra were acquired on a Bruker Avance 500 spectrometer (500.0 MHz for ${ }^{1} \mathrm{H}$ and $125.7 \mathrm{MHz}$ for ${ }^{13} \mathrm{C}$ ) in dry $\mathrm{CDCl}_{3} . \mathrm{CDCl}_{3}$ was dried prior to use by anhydrous $\mathrm{K}_{2} \mathrm{CO}_{3}$ and distilled. The measurements were performed in an oven-dried NMR tube, which was sealed after sample preparation. The concentration of the samples was 38 $\mathrm{mM}$ and $40 \mathrm{mM}$ for the degradation kinetics and kinetics of reaction with amines, respectively. All of the kinetic measurements were performed at $25^{\circ} \mathrm{C}$. Temperature calibration was done using the standard method with an $\mathrm{MeOH}$ sample. ${ }^{1} \mathrm{H}$ and ${ }^{13} \mathrm{C}$ resonances were assigned based on PFG H,C-HSQC and H,C-HMBC experiments (for numbering see Supportin Info). ${ }^{1} \mathrm{H}$ and ${ }^{13} \mathrm{C}$ NMR of compounds 7a-d have been already reported. ${ }^{34}$

Computational Details: All the density functional theory (DFT) calculations reported in the study were carried out using the Turbomole 6.0 program. ${ }^{36}$ The Perdew-Burke-Ernzerhof $(\mathrm{PBE})^{37}$ and hybrid three-parameter Becke's ${ }^{38}$ (B3LYP) functionals were used throughout. The calculations were expedited by expanding the Coulomb integrals in an auxiliary basis set, the resolution-of-identity (RI-J) approximation. ${ }^{39-40}$ All the geometry optimisations were conducted using the def2-SVP basis set, ${ }^{41}$ whereas the single-point energies were recomputed in the def2-TZVP (triple-zeta valence with two polarisation functions on each atom). ${ }^{42}$

To account for the solvation effects, the conductor-like screening model (COSMO) method ${ }^{41-}$ ${ }^{42}$ was used with the dielectric constant corresponding to chloroform $\left(\varepsilon_{\mathrm{r}}=4.9\right)$. In order to account for dispersion, we used the DFT + D method (i.e. the DFT method with the empirical dispersion terms) available in Turbomole $6.0 .^{43}$ The Gibbs free energy was then calculated as the sum of these contributions:

$G=E_{\mathrm{el}}+G_{\mathrm{solv}}+E_{\mathrm{ZPE}}-R T \ln \left(q_{\mathrm{trans}} q_{\mathrm{rot}} q_{\mathrm{vib}}\right)$

where $E_{\mathrm{el}}$ is the in vacuo energy of the system (at the B3LYP/def2-TZVP level and the geometry optimised at the RI-PBE/def2-SVP level), $G_{\text {solv }}$ is the solvation free energy (at the $\mathrm{RI}-\mathrm{PBE} / \mathrm{def} 2-\mathrm{SVP}$ level$), E_{\mathrm{ZPE}}$ is the zero-point energy, and $-R T \ln \left(q_{\text {trans }} q_{\mathrm{rot}} q_{\mathrm{vib}}\right)$ accounts for the entropic terms and the thermal correction to the enthalpy obtained from a frequency 
calculation using the same method and software as for the geometry optimisation at the RIPBE/def2-SV(P) level, $298 \mathrm{~K}$, and 1 atm using the ideal-gas approximation. ${ }^{44}$

\section{(E)-3-Ethoxy-2-methylacryloyl isocyanate $(3 b)$}

${ }^{1} \mathrm{H}$ NMR $\left(\mathrm{CDCl}_{3}, 500.0 \mathrm{MHz}\right): 1.36 \mathrm{t}, 3 \mathrm{H}, J\left(\mathrm{CH}_{3}, \mathrm{CH}_{2}\right)=7.1\left(\mathrm{CH}_{3} \mathrm{CH}_{2} \mathrm{O}\right) ; 1.82 \mathrm{~d}, 3 \mathrm{H}$, $J\left(\mathrm{CH}_{3}, \mathrm{CH}\right)=1.2\left(\mathrm{CH}_{3}\right) ; 4.13 \mathrm{q}, 2 \mathrm{H}, J\left(\mathrm{CH}_{2}, \mathrm{CH}_{3}\right)=7.1\left(\mathrm{OCH}_{2} \mathrm{CH}_{3}\right) ; 7.53 \mathrm{q}, 1 \mathrm{H}, J\left(\mathrm{CH}, \mathrm{CH}_{3}\right)$ $=1.2(\mathrm{CH}=) \cdot{ }^{13} \mathrm{C}$ NMR $\left(\mathrm{CDCl}_{3}, 125.7 \mathrm{MHz}\right): 8.79\left(\mathrm{CH}_{3}\right) ; 15.34\left(\mathrm{CH}_{3} \mathrm{CH}_{2} \mathrm{O}\right) ; 70.98$ $\left(\mathrm{OCH}_{2} \mathrm{CH}_{3}\right) ; 110.19(\mathrm{C}=) ; 130.47(\mathrm{NCO}) ; 162.82(\mathrm{CH}=) ; 165.52(\mathrm{CO})$.

\section{1-(2,4,6-Tri-tert-butylphenyl)-3-((E)-3-ethoxyacryloyl)urea (11a)}

${ }^{1} \mathrm{H}$ NMR $\left(\mathrm{CDCl}_{3}, 500.0 \mathrm{MHz}\right): 1.30 \mathrm{~s}, 9 \mathrm{H}\left(\left(\mathrm{CH}_{3}\right)_{3} \mathrm{C}^{-} 4^{\prime}\right) ; 1.32 \mathrm{t}, 3 \mathrm{H}, J\left(\mathrm{CH}_{3}, \mathrm{CH}_{2}\right)=7.1$ $\left(\mathrm{CH}_{3} \mathrm{CH}_{2} \mathrm{O}\right) ; 1.40 \mathrm{~s}, 18 \mathrm{H}\left(\left(\mathrm{CH}_{3}\right)_{3} \mathrm{C}^{-2} 2^{\prime}, 6^{\prime}\right) ; 3.90 \mathrm{q}, 2 \mathrm{H}, J\left(\mathrm{CH}_{2}, \mathrm{CH}_{3}\right)=7.1\left(\mathrm{OCH}_{2} \mathrm{CH}_{3}\right) ; 5.26$ $\mathrm{d}, 1 \mathrm{H}, J(5,6)=12.2(\mathrm{H}-5) ; 7.40 \mathrm{~s}, 2 \mathrm{H},\left(\mathrm{H}^{\prime} 3^{\prime}, 5^{\prime}\right) ; 7.72 \mathrm{~d}, 1 \mathrm{H}, J(6,5)=12.2(\mathrm{H}-6) ; 8.54 \mathrm{bs}, 1$ $\mathrm{H}(\mathrm{H}-3) ; 10.29 \mathrm{bs}, 1 \mathrm{H}(\mathrm{H}-1) .{ }^{13} \mathrm{C}$ NMR $\left(\mathrm{CDCl}_{3}, 125.7 \mathrm{MHz}\right): 14.48\left(\mathrm{CH}_{3} \mathrm{CH}_{2} \mathrm{O}\right) ; 31.38$ $\left(\left(\mathbf{C H}_{3}\right)_{3} \mathrm{C}^{-} 4^{\prime}\right) ; 31.86\left(\left(\mathrm{CH}_{3}\right)_{3} \mathrm{C}-2^{\prime}, 6^{\prime}\right) ; 34.97 \quad\left(\left(\mathrm{CH}_{3}\right)_{3} \mathbf{C}^{\prime}-4^{\prime}\right) ; 36.19 \quad\left(\left(\mathrm{CH}_{3}\right)_{3} \mathbf{C}^{\prime} 2^{\prime}, 6^{\prime}\right) ; 67.87$ $\left(\mathrm{OCH}_{2} \mathrm{CH}_{3}\right) ; 97.48$ (C-5); $122.93\left(\mathrm{C}-3^{\prime}, 5^{\prime}\right) ; 129.26$ (C-1'); 148.01 (C-2’,6’); 149.55 (C-4'); 154.77 (C-2); 163.84 (C-6); 168.26 (C-4).

HR-MS for $\mathrm{C}_{24} \mathrm{H}_{39} \mathrm{~N}_{2} \mathrm{O}_{3}(\mathrm{M}+\mathrm{H})^{+}$calcd 403.2955, found 403.2955.

\section{1-(2,4,6-Tri-tert-butylphenyl)-3-((E)-3-ethoxy-2-methylacryloyl)urea (11b)}

${ }^{1} \mathrm{H}$ NMR $\left(\mathrm{CDCl}_{3}, 500.0 \mathrm{MHz}\right): 1.31 \mathrm{~s}, 9 \mathrm{H}\left(\left(\mathrm{CH}_{3}\right)_{3} \mathrm{C}_{-4} 4^{\prime}\right) ; 1.33 \mathrm{t}, 3 \mathrm{H}, J\left(\mathrm{CH}_{3}, \mathrm{CH}_{2}\right)=7.1$ $\left(\mathrm{CH}_{3} \mathrm{CH}_{2} \mathrm{O}\right) ; 1.42 \mathrm{~s}, 18 \mathrm{H}\left(\left(\mathrm{CH}_{3}\right)_{3} \mathrm{C}-2^{\prime}, 6^{\prime}\right) ; 1.84 \mathrm{~d}, 3 \mathrm{H}, J\left(\mathrm{CH}_{3}, 6\right)=1.2\left(5-\mathrm{CH}_{3}\right) ; 4.04 \mathrm{q}, 2 \mathrm{H}$, $J\left(\mathrm{CH}_{2}, \mathrm{CH}_{3}\right)=7.1\left(\mathrm{OCH}_{2} \mathrm{CH}_{3}\right) ; 7.43 \mathrm{~s}, 2 \mathrm{H},\left(\mathrm{H}^{\prime} 3^{\prime}, 5^{\prime}\right) ; 7.58 \mathrm{q}, 1 \mathrm{H}, J\left(6, \mathrm{CH}_{3}\right)=1.2(\mathrm{H}-6) ; 7.95$ bs, $1 \mathrm{H}(\mathrm{H}-3) ; 10.45 \mathrm{bs}, 1 \mathrm{H}(\mathrm{H}-1) .{ }^{13} \mathrm{C} \mathrm{NMR}\left(\mathrm{CDCl}_{3}, 125.7 \mathrm{MHz}\right): 8.83\left(5-\mathrm{CH}_{3}\right) ; 15.44$ $\left.\left(\mathrm{CH}_{3} \mathrm{CH}_{2} \mathrm{O}\right) ; 31.37\left(\left(\mathrm{CH}_{3}\right)_{3} \mathrm{C}^{\prime} 4^{\prime}\right) ; 31.87\left(\left(\mathrm{CH}_{3}\right)_{3} \mathrm{C}-2^{\prime}\right)^{\prime}\right) ; 34.98\left(\left(\mathrm{CH}_{3}\right)_{3} \mathrm{C}^{\prime}-4^{\prime}\right) ; 36.20\left(\left(\mathrm{CH}_{3}\right)_{3} \mathrm{C}-\right.$ 2',6'); $70.54\left(\mathrm{OCH}_{2} \mathrm{CH}_{3}\right) ; 105.68$ (C-5); $123.04\left(\mathrm{C}-3^{\prime}, 5^{\prime}\right) ; 129.30$ (C-1'); 147.97 (C-2',6' $)$; 149.53 (C-4'); 154.33 (C-2); 158.71 (C-6); 169.64 (C-4).

HR-MS for $\mathrm{C}_{25} \mathrm{H}_{41} \mathrm{~N}_{2} \mathrm{O}_{3}(\mathrm{M}+\mathrm{H})^{+}$calcd 417.3112, found 417.3111.

\section{Methyl (E)-3-ethoxy-2-methylacryloylcarbamate (12)}

${ }^{1} \mathrm{H}$ NMR $\left(\mathrm{CDCl}_{3}, 500.0 \mathrm{MHz}\right): 1.33 \mathrm{t}, 3 \mathrm{H}, J\left(\mathrm{CH}_{3}, \mathrm{CH}_{2}\right)=7.1\left(\mathrm{CH}_{3} \mathrm{CH}_{2} \mathrm{O}\right) ; 1.83 \mathrm{~d}, 3 \mathrm{H}$, $J\left(\mathrm{CH}_{3}, 6\right)=1.2\left(5-\mathrm{CH}_{3}\right) ; 3.79 \mathrm{~s}, 3 \mathrm{H}\left(\mathrm{CH}_{3} \mathrm{O}\right) ; 4.08 \mathrm{q}, 2 \mathrm{H}, J\left(\mathrm{CH}_{2}, \mathrm{CH}_{3}\right)=7.1\left(\mathrm{OCH}_{2} \mathrm{CH}_{3}\right) ; 7.42$

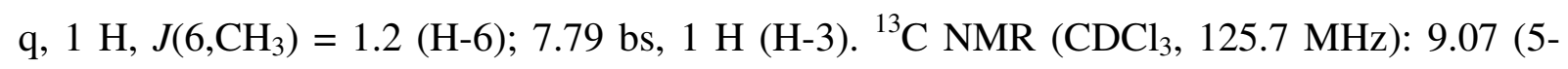


$\left.\mathrm{CH}_{3}\right) ; 15.26\left(\mathrm{CH}_{3} \mathrm{CH}_{2} \mathrm{O}\right) ; 52.72\left(\mathrm{CH}_{3} \mathrm{O}\right) ; 70.12\left(\mathrm{OCH}_{2} \mathrm{CH}_{3}\right) ; 107.09$ (C-5); 151.93 (C-2); 157.59 (C-6); 165.97 (C-4).

HR-MS for $\mathrm{C}_{8} \mathrm{H}_{13} \mathrm{NO}_{4} \mathrm{Na}(\mathrm{M}+\mathrm{H}+\mathrm{Na})^{+}$calcd 210.0742, found 210.0741 .

Supporting Information (see footnote on the first page of this article): Kinetics measurements and computational data including .

\section{Acknowledgments}

We gratefully acknowledge the financial support by the Ministry of Education, Youth, and Sports of the Czech Republic (Research projects Z40550506, 2B06065, and LC512) and the Ministry of Health (Grant NR/9138 - 3).

[1] Galmarini C. M., Mackey J. R.,Dumontet C. The Lancet Oncology 2002, 3( 7), 415 424.

[2] Miura S, Izuta S. Curr. Drug. Targets. 2004, 5(2), 191-195.

[3] Simons C., Wu Q., Htar T.T. Curr. Top. Med. Chem. 2005, (13), 1191-1203.

[4] Schneller, S.W. Curr. Top. Med. Chem. 2002, 2(10), 1087-1092.

[5] Jeong L.S., Lee J.A. Antivir. Chem. Chemother. 2004, 15(5), 235-250.

[6] Holý A. Antiviral Res. 2006, 71(2-3), 248-253.

[7] Rejman D., Masojídková M., De Clercq E., Rosenberg I.: Nucleosides \& Nucleotides 2001, 20 (8) 1497-1522.

[8] Kočalka, P.; Pohl, R.; Rejman, D.; Rosenberg, I. Tetrahedron 2006, 62, 5763-5774

[9] Rejman D., Kočalka P., Buděšínský M., Pohl R., Rosenberg I.: Tetrahedron 2007, 63, $5,1243-1253$.

[10] Yamada K., Sakata S., Yoshimura Y. J. Org. Chem. 1998, 63, 6891-6899.

[11] Lu W., Sengupta S., Petersen J. L., Akhmedov N. G., Shi X. J. Org. Chem. 2007, 72, 5012-5015.

[12] Michel B. Y., Strazewski P. Tetrahedron 2007, 63, 9836-9841.

[13] Abrams, H. M.; Ho, L.; Chu, S. H. Journal of Heterocyclic Chemistry 1981, 18, 947 951.

[14] Maruyama, Tokumi; Kozai, Shigetada; Uchida, Mayuko Nucleosides \& Nucleotides 1999, 18(4-5), $661-672$.

[15] Takenaka, Keiko; Muraoka, Masako; Tsuji, Tadakazu Journal of Heterocyclic Chemistry 1997, 34(2), 669 - 673. 
[16] Shaw G., Warrener R. N. J. Chem. Soc.1958, 153-156. Shaw G., Warrener R. N. J. Chem. Soc. 1958, 157-161.

[17] Shealy Y. F., O’Dell C. A.: J. Heterocyclic Chem. 1976, 13, 1015-1020.

[18] Wyatt P. G., Anslow A. S., Coomber B. A., Cousins R. P. C., Evans D. N., Gilbert V. S., Humber D. C., Paternoster I. L., Sollis S. L., Tapolczay D. J., Weingarten G. G.: Nucleosides Nucleotides 1995, 14, 2039-2049.

[19] Hřebabecký H., Masojídková M., Holý A.: Collect. Czech. Chem. Commun. 2005, 70, 519-538.

[20] Csuk R., von Scholz Y.: Tetrahedron 1995, 51, 7193-7206.

[21] Borthwick A. D., Evans D. N., Kirk B. E., Biggadike K., Exal A. M., Youds P., Roberts S. M., Knight D. J., Coates A. V.: J. Med. Chem. 1990, 33, 179-186.

[22] Bodenteich M., Marquez V. E., Barchi J. J., Hallows W. H., Goldstein B. M., Driscoll J. S.: J. Org. Chem. 1993, 58, 6009-6015.

[23] Hosono F., Nishiyama S., Yamamura S.: Tetrahedron, 1994, 50, 47, 13335-13346.

[24] Raganathan S., George K. S.: Tetrahedron 1997, 53, 9, 3347-3362.

[25] Miyabe H., Kanehira S., Kume K., Kandori H., Naito T.: Tetrahedron, 1998, 54, 5883-5892.

[26] Moon H. R., Kim H. O., Chun M. W., Jeong L. S.: J. Org. Chem.. 1999, 64, 47334741.

[27] Wang P., Gullen B., Newton M. G., Cheng Y. C., Shinazi R. F., Chu C.K.: J. Med. Chem. 1999, 42, 3390-3399.

[28] Bera S., Mickle T., Nair V.: Nucleosides Nucleotides 1999, 18 (11\&12), 2379-2395.

[29] Kim H. S., Ravi R. G., Marquez V. E., Maddileti S., Wihlborg A. K., Erlinge D., Malmsjö M., Boyer J. L., Harden T. K., Jacobson K. A.: J. Med. Chem. 2002, 45, 208-218.

[30] Nieto M. I., Caamaño O., Fernández F., Gómez M., Balzarini J., DeClercq E.: Nucleosides Nucleotides 2002, 21 (3), 243-255.

[31] Kim S. A., Lee H. M., Ryu J. S., Kim H. S.: Synlett 2007, 7, 1055-1058.

[32] Migliore M. D., Zonta N., McGuigan C., Henson G., Andrei G., Snoeck R., Balzarini J.: J. Med. Chem. 2007, 50, 6485-6492.

[33] Shealy Y. F., O’Dell C. A.: J. Heterocyclic Chem. 1976, 13, 1041-1047.

[34] Rejman D., Kovačková S., Pohl R., Dračínský M., Fiedler P., Rosenberg I. Tetrahedron, 2009, 65, 41, 8513-8523.

[35] Harvey, J. N. Faraday Discuss. 2009, 145, 487-505. 
[36] R. Ahlrichs, M. Bär, M. Häser, H. Horn, C. Kölmel, Chem. Phys. Lett. 1989 162, 165169.

[37] J. P. Perdew, K. Burke, M. Ernzerhof, Phys. Rev. Lett., 1996, 77, 3865-3868.

[38] (a) A. D. Becke, Phys. Rev. A 1988, 38, 3098-3100. (b) C. T. Lee, W. T. Yang, R. G. Parr, Phys. Rev. B 1988, 37, 785-789. (c) A. D. Becke, J. Chem. Phys. 1993, 98, 5648-5652. (d) P. J. Stephens, F. J. Devlin, C. F. Chabalowski, M. J. Frisch, J. Phys. Chem. 1994, 98, 11623-11627.

[39] K. Eichkorn, O. Treutler, H. Öhm, M. Häser, R. Ahlrichs, Chem. Phys. Lett. 1995, 240, 283-290.

[40] K. Eichkorn, F. Weigen, O. Treutler, R. Ahlrichs, Theor. Chim. Acta 1997, 97, 119124.

[41] (a) A. Schäfer, H. Horn, R. Ahlrichs, J. Chem. Phys. 1992, 97, 2571-2577. (b) F. Weigend, R. Ahlrichs, Phys. Chem. Chem. Phys. 2006, 7, 3297-3305.

[42] A. Klamt, G. Schuurmann, J. Chem. Soc.-Perkin Trans. 2 1993, 799-805.

[42] A. Schäfer, A. Klamt, D. Sattel, J. C. W. Lohrenz, F. Eckert, Phys. Chem. Chem. Phys. 2000, 2, 2187-2193.

[43] Grimme, S. J Comput Chem 2004, 25, 1463-1473.

[44] Jensen, F. Introduction to Computational Chemistry; John Wiley \& Sons: New York, 1999. 
Table 1 . The kinetics of $\mathbf{7 a - d}$ degradation monitored by ${ }^{1} \mathrm{H}$ NMR.

[a] $k_{+}=1.83 k_{-}$

\begin{tabular}{lllll}
\hline Degradation & Reaction & Kinetics & $k\left(\mathrm{hr}^{-1}\right)$ & $t_{1 / 2}(\mathrm{hr})$ \\
\hline $\mathbf{7 a}$ & reversible & first-order & $3.00 \times 10^{-2[a]}$ & 23.1 \\
$\mathbf{7 b}$ & irreversible & first-order & 2.32 & 0.30 \\
$\mathbf{7 c}$ & no degradation observed & & \\
$\mathbf{7 d}$ & no degradation observed & & \\
\hline
\end{tabular}


Table 2. The theoretical calculations of reaction free energies and activation barriers carried out using the B3LYP/def2-TZVP//RI-PBE/def2-SVP method. The solvation effects were included through COSMO calculations and the energies corrected for zero-point energies, thermal corrections to enthalpy and entropic terms (using ideal gas approximation) to obtain free energy estimates. The experimental data are shown in the right part of the table $\left(\Delta G_{\text {exp }}^{\ddagger}\right.$ were calculated using Eyring equation: $\left.\Delta G^{\ddagger}=R T[23.76-\ln (k / T)]\right)$.

\begin{tabular}{lllll}
\hline Degradation & $\begin{array}{l}\Delta G_{\mathrm{calc}} \\
\left(\mathrm{kJ} \cdot \mathrm{mol}^{-1}\right)\end{array}$ & $\begin{array}{l}\Delta G_{\mathrm{calc}}^{\ddagger} \\
\left(\mathrm{kJ}_{\mathrm{mol}}{ }^{-1}\right)\end{array}$ & $\begin{array}{l}k_{\exp } \\
\left(\mathrm{s}^{-1}\right)\end{array}$ & $\begin{array}{l}\Delta G_{\text {exp }}^{\ddagger} \\
\left(\mathrm{kJ} \cdot \mathrm{mol}^{-1}\right)\end{array}$ \\
\hline $\mathbf{7 a}$ & -24.6 & 84.1 & $8.33 \times 10^{-6}$ & 102.0 \\
$\mathbf{7 b}$ & -30.2 & 72.0 & $6.44 \times 10^{-4}$ & 91.2 \\
$\mathbf{7 c}$ & -3.9 & 88.4 & - & - \\
$\mathbf{7 d}$ & -15.1 & 79.1 & - & - \\
\hline
\end{tabular}


Table 3. The kinetics of the reaction of the reagents 7a-d with 2,4,6-tri-tert-butylaniline in dry $\mathrm{CDCl}_{3}$ at $25^{\circ} \mathrm{C}$.

\begin{tabular}{lll}
\hline Reagent & $k\left(\mathrm{hr}^{-1}\right)$ & $t_{1 / 2}(\mathrm{hr})$ \\
\hline $\mathbf{7 a}$ & $2.7 \times 10^{-2}$ & 25.7 \\
$7 \mathbf{b}$ & 1.2 & 0.6 \\
$\mathbf{7 c}$ & $7.4 \times 10^{-2}$ & 9.4 \\
$\mathbf{7 d}$ & $2.7 \times 10^{-1}$ & 2.6 \\
\hline
\end{tabular}




\section{Supporting Information}

The numbering for NMR assignment:

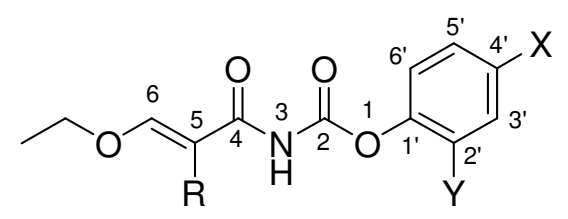

7a: $\mathrm{R}=\mathrm{H}, \mathrm{X}=\mathrm{H}, \mathrm{Y}=\mathrm{NO}_{2}$

7b: $R=\mathrm{CH}_{3}, X=\mathrm{H}, \mathrm{Y}=\mathrm{NO}_{2}$

7c: $\mathrm{R}=\mathrm{H}, \mathrm{X}=\mathrm{NO}_{2}, \mathrm{Y}=\mathrm{H}$

7d: $\mathrm{R}=\mathrm{CH}_{3}, \mathrm{X}=\mathrm{NO}_{2}, \mathrm{Y}=\mathrm{H}$

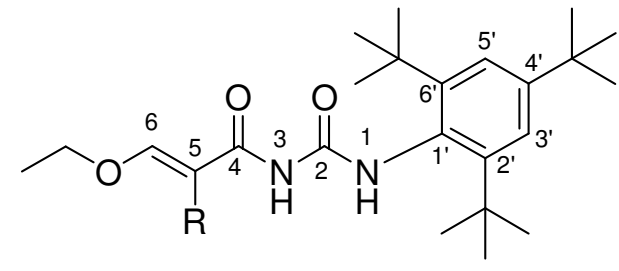

11a: $\mathrm{R}=\mathrm{H}$

11b: $\mathrm{R}=\mathrm{CH}_{3}$

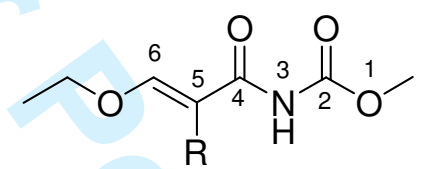

12 

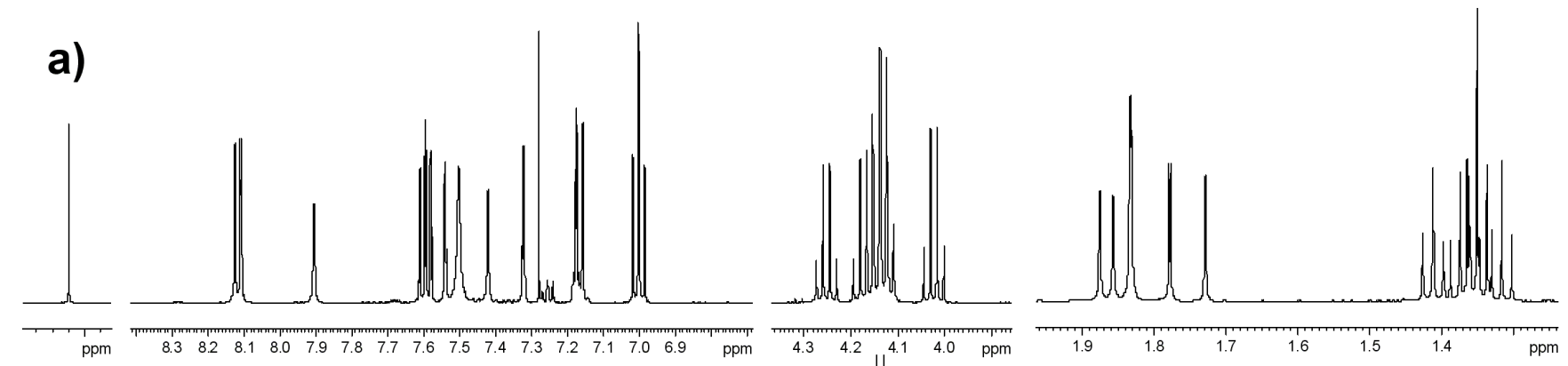

b)
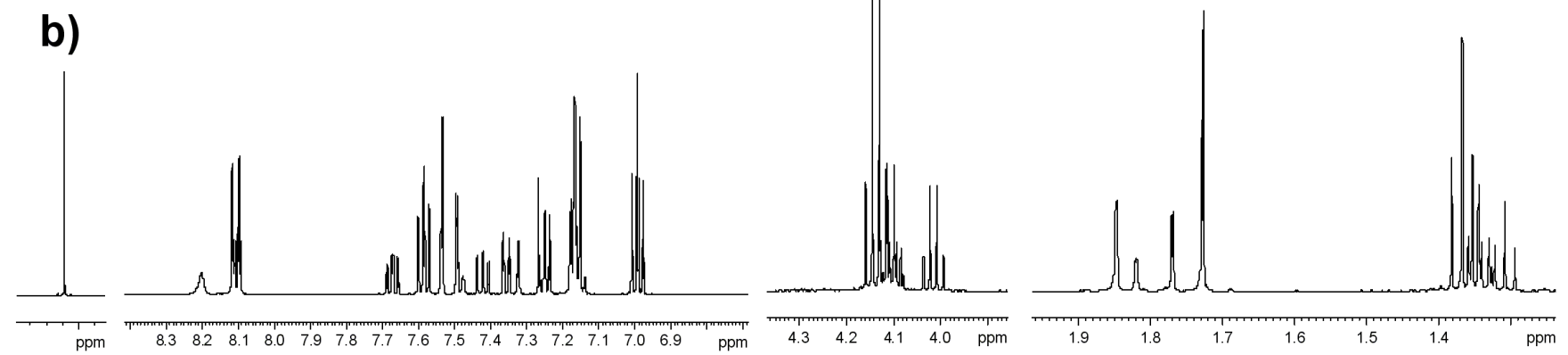

c)
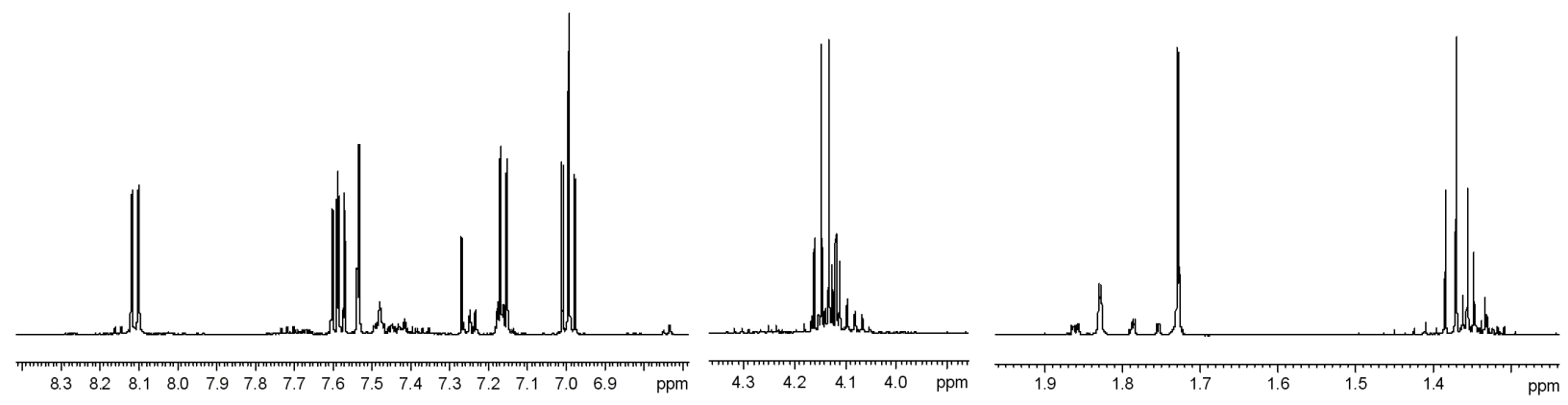

Fig.1. a) The ${ }^{1} \mathrm{H}$ NMR of $\mathbf{7 b}$ recorded in commercial grade $\mathrm{CDCl}_{3}$. b) The ${ }^{1} \mathrm{H} \mathrm{NMR}$ of $\mathbf{7 b}$ recorded in dry $\mathrm{CDCl}_{3}$ right after dissolution $\left(t_{\mathrm{r}}=5 \mathrm{~min}\right)$. c) The same mixture as b) after 2 hours. 
<smiles>CCO/C=C(\C)C(=O)NC(=O)Oc1ccccc1[N+](=O)[O-]</smiles>
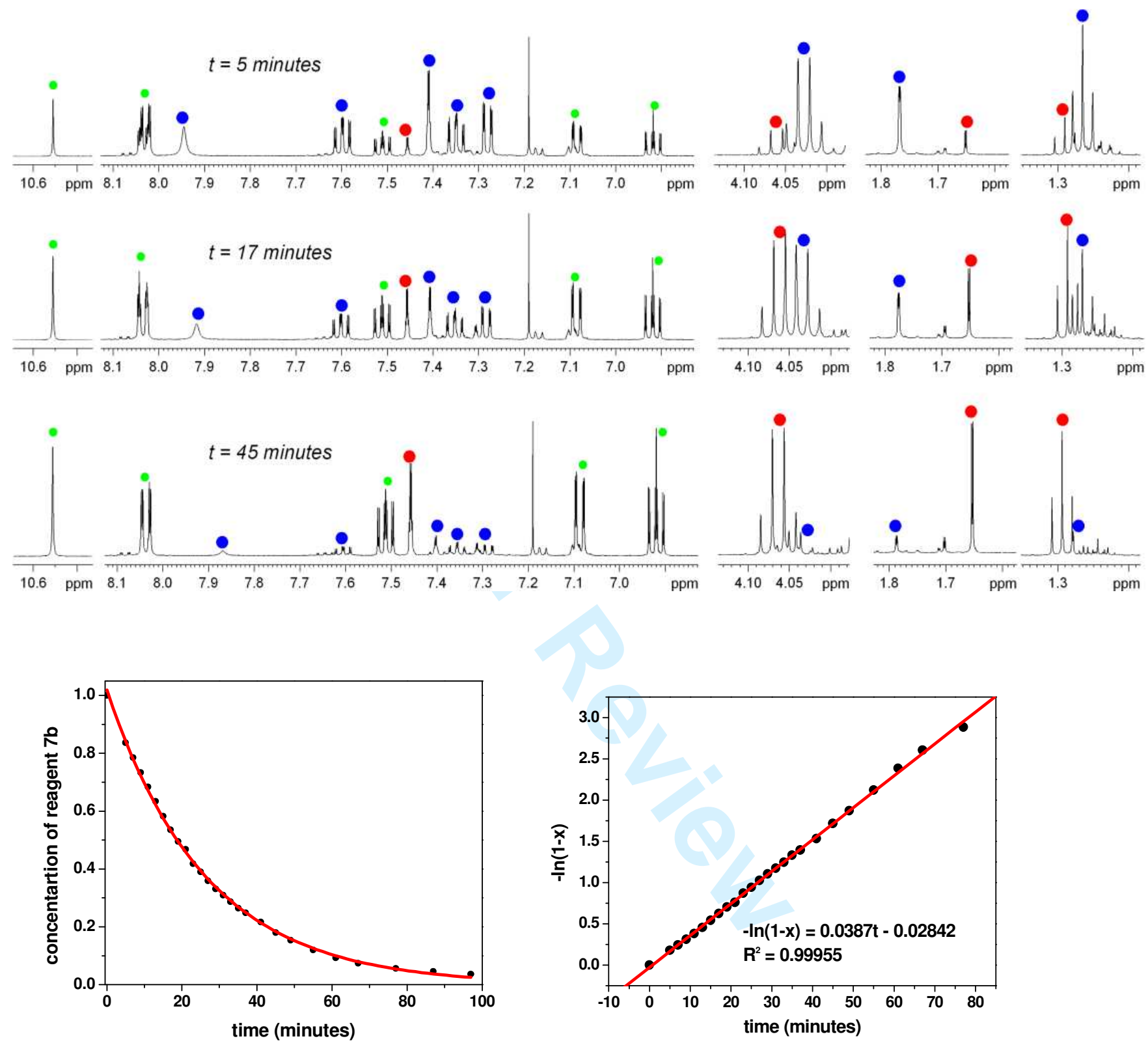

Fig. 2. The kinetics of the decomposition of $\mathbf{7 b}$ in dry $\mathrm{CDCl}_{3}$ in a sealed NMR tube at $25{ }^{\circ} \mathrm{C}$. 
<smiles>CCOC=CC(=O)NC(=O)Oc1ccccc1[N+](=O)[O-]</smiles>

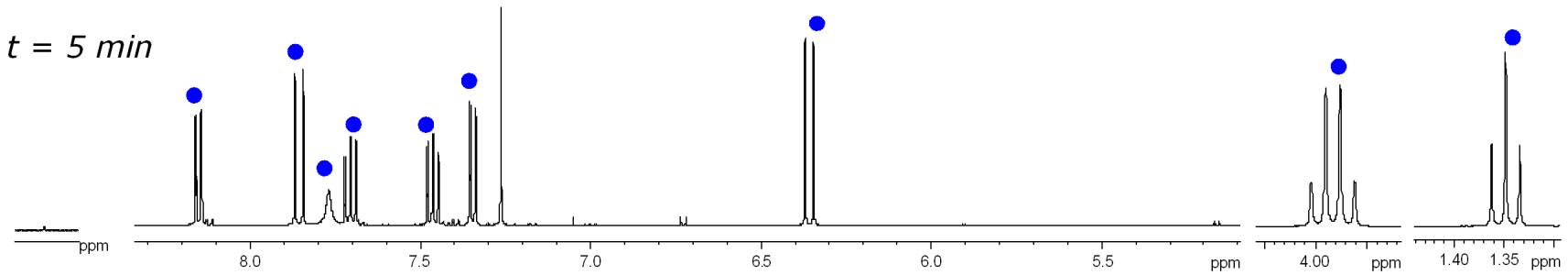

$t=16$ hours

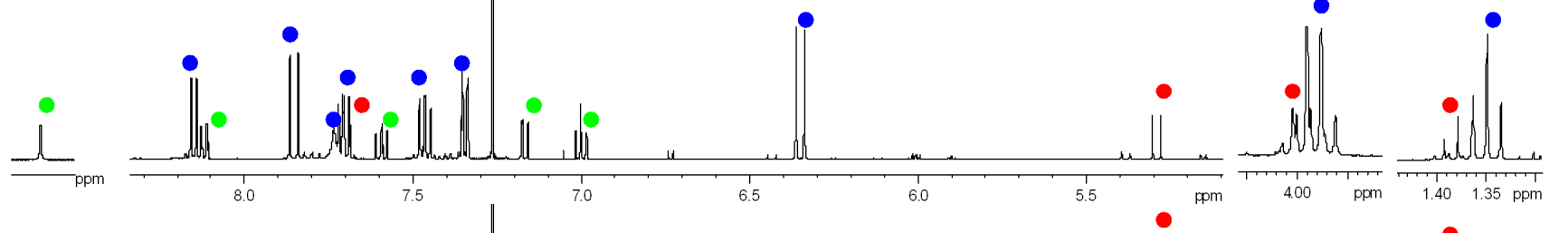

\section{$t=121$ hours}
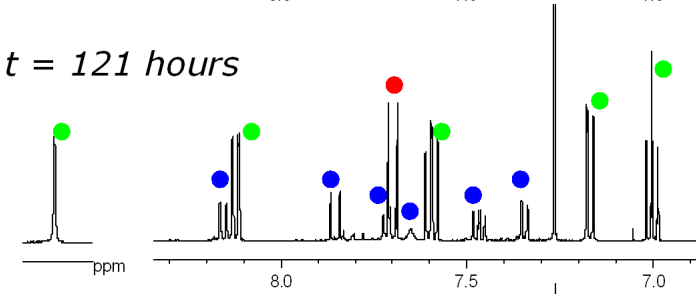

$t=157$ hours
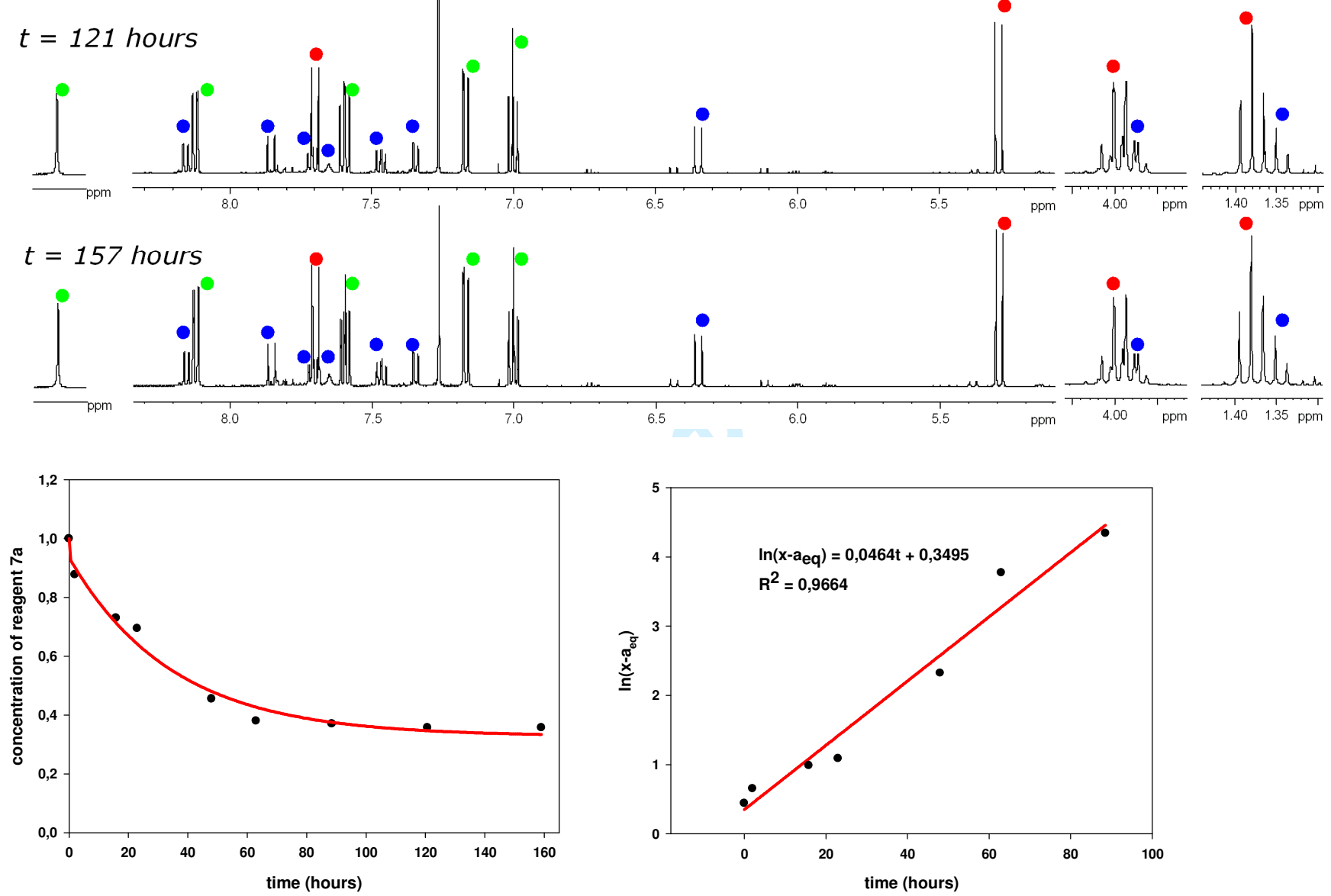

Fig. 3. The kinetics of the decomposition of $\mathbf{7 a}$ in dry $\mathrm{CDCl}_{3}$ in a sealed NMR tube at $25^{\circ} \mathrm{C}$. 


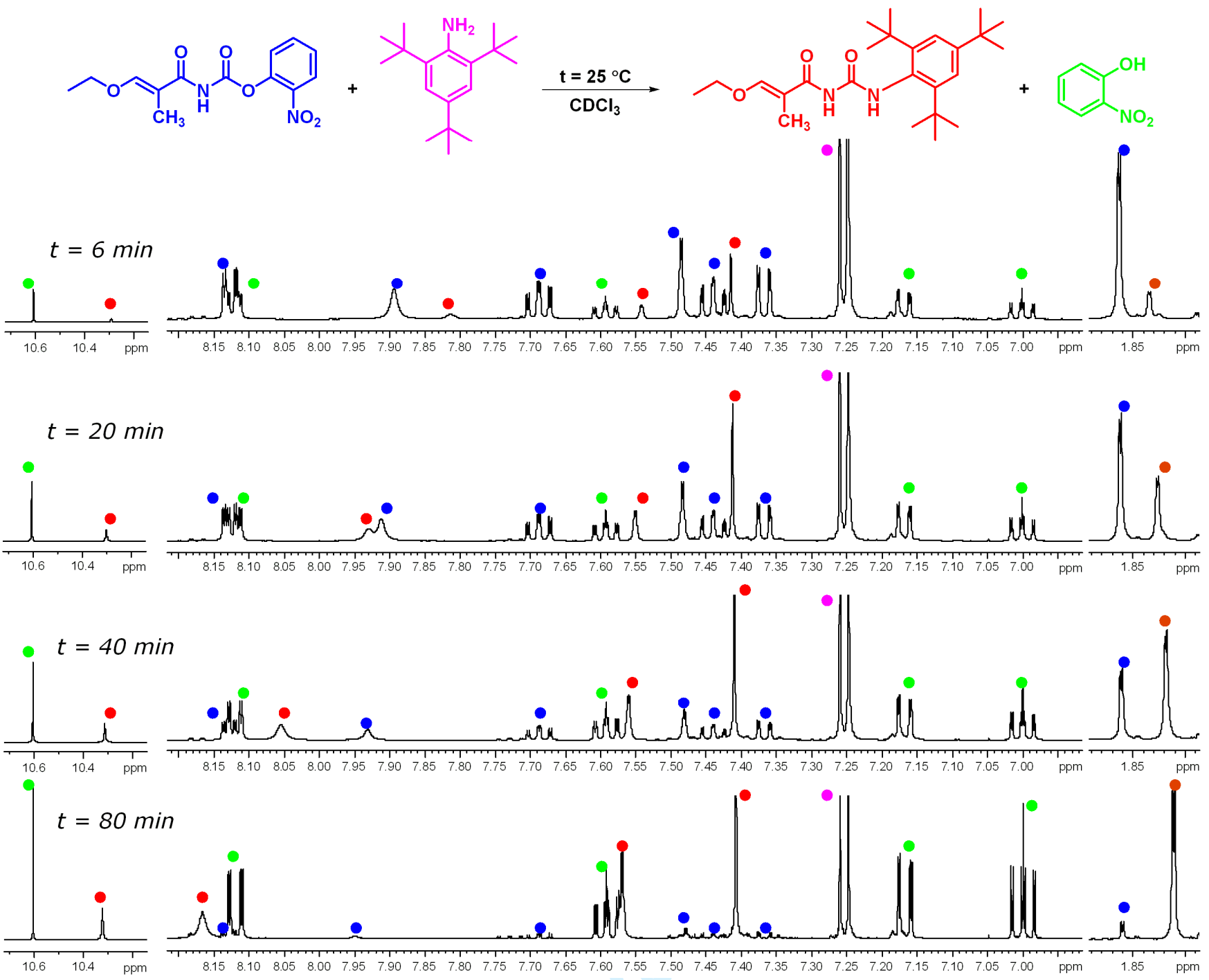

Fig. 4. The course of the reaction of $7 \mathbf{b}$ with 2,4,6-tri-tert-butylaniline in dry $\mathrm{CDCl}_{3}$ in a sealed NMR tube at $25^{\circ} \mathrm{C}$. 
Table S1: The molecular energies, contributions to free energy and the difference in the total energies and free energies for 7a. If not explicitly mentioned, the energies are in atomic units (a.u.).

\begin{tabular}{|c|c|c|c|c|c|c|}
\hline $\begin{array}{l}\text { Conformer } \\
\text { (Structure) }\end{array}$ & $\begin{array}{l}E(P B E) \\
\text { def2-SVP }\end{array}$ & $\begin{array}{l}E(B 3 L Y P) \\
\text { def2-TZVP }\end{array}$ & $\begin{array}{l}E(\mathrm{PBE}+\mathrm{D}+ \\
\text { COSMO) } \\
\text { def2-SVP }\end{array}$ & $G_{\text {tot }}$ & $\begin{array}{l}\Delta \boldsymbol{G} \\
\left(\mathrm{kJ}^{\prime} \mathrm{mol}^{-1}\right)\end{array}$ & $\begin{array}{l}\Delta \boldsymbol{E} \\
\left(\mathrm{kJ} \cdot \mathrm{mol}^{-1}\right)\end{array}$ \\
\hline 2NH-EEEE & -1023.33987 & -1025.10724 & -1023.38280 & -1024.97035 & 34.2 & 40.6 \\
\hline 2NH-EEEZ & -1023.34859 & -1025.11567 & -1023.39021 & -1024.97587 & 19.7 & 18.5 \\
\hline 2NH-EEZE & -1023.34009 & -1025.11241 & -1023.38208 & -1024.97643 & 18.2 & 27.1 \\
\hline 2NH-EEZZ & -1023.34039 & -1025.11208 & -1023.38195 & -1024.97460 & 23.0 & 28.0 \\
\hline 2NH-EZEE & -1023.34690 & -1025.11762 & -1023.38786 & -1024.98036 & 7.9 & 13.4 \\
\hline 2NH-EZEZ & -1023.35190 & -1025.12273 & -1023.39140 & -1024.98337 & $\mathbf{0 . 0}$ & 0.0 \\
\hline 2NH-EZZE & -1023.34505 & -1025.11667 & -1023.38653 & -1024.98032 & 8.0 & 15.9 \\
\hline 2NH-EZZZ & -1023.34454 & -1025.11661 & -1023.38661 & -1024.98184 & 4.0 & 16.1 \\
\hline 2NH-ZEEE & -1023.33878 & -1025.10532 & -1023.38510 & -1024.96851 & 39.0 & 45.7 \\
\hline 2NH-ZEEZ & -1023.34170 & -1025.11103 & -1023.38449 & -1024.97348 & 25.9 & 30.7 \\
\hline 2NH-ZEZE & -1023.34533 & -1025.11548 & -1023.38694 & -1024.97854 & 12.7 & 19.0 \\
\hline 2NH-ZEZZ & -1023.34843 & -1025.11507 & -1023.39039 & -1024.97655 & 17.9 & 20.1 \\
\hline 2NH-ZZEE & -1023.34136 & -1025.11166 & -1023.38333 & -1024.97520 & 21.4 & 29.0 \\
\hline 2NH-ZZEZ & -1023.34660 & -1025.11717 & -1023.38694 & -1024.97938 & 10.5 & 14.6 \\
\hline 2NH-ZZZE & -1023.33888 & -1025.11032 & -1023.38172 & -1024.97333 & 26.4 & 32.6 \\
\hline 2NH-ZZZZ & -1023.33844 & -1025.11021 & -1023.38171 & -1024.97799 & 14.1 & 32.9 \\
\hline \multicolumn{7}{|l|}{ enol-form } \\
\hline 3OH-EEZE & -1023.33077 & -1025.10253 & -1023.36969 & -1024.96243 & $\mathbf{5 5 . 0}$ & 53.0 \\
\hline 3OH-EZZE & -1023.33072 & -1025.10242 & -1023.36865 & -1024.96292 & 53.7 & 53.3 \\
\hline \multicolumn{7}{|c|}{ 6-Membered Ring Transition States Corresponding to the $3 \mathrm{OH} \rightarrow$ Phenol Hydrogen Transfer } \\
\hline EEZE & -1023.32247 & -1025.08896 & -1023.36016 & -1024.95135 & 84.1 & 88.7 \\
\hline EZZE & -1023.32191 & -1025.08868 & -1023.35961 & -1024.95117 & 84.5 & 89.4 \\
\hline \multicolumn{7}{|c|}{ Product Complex } \\
\hline EEZE & -1023.34109 & -1025.11742 & -1023.37758 & -1024.98113 & 5.9 & 13.9 \\
\hline $\mathrm{EZZE}$ & -1023.34171 & -1025.11767 & -1023.37771 & -1024.98169 & 4.4 & 13.3 \\
\hline
\end{tabular}

$E(\mathrm{PBE}) \ldots$ in vacuo energy of the system optimised at the RI-PBE/def2-SVP level of theory

$E(B 3 L Y P)$ in vacuo energy of the system calculated at the B3LYP/def2-TZVP//RI-PBE/def2-SVP level

$E(\mathrm{PBE}+\mathrm{D}+\mathrm{COSMO})$ energy of the system optimised at the RI-PBE+D+COSMO/def2-SVP level, i.e. with the empirical dispersion correction and COSMO solvation model

$G_{\mathrm{tot}}=E(\mathrm{~B} 3 \mathrm{LYP})+E(\mathrm{PBE}+\mathrm{D}+\mathrm{COSMO})-E(\mathrm{PBE})+E_{\mathrm{ZPE}}-R T \ln \left(q_{\text {trans }} q_{\mathrm{rot}} q_{\mathrm{vib}}\right)$

$\Delta G$...relative difference in free energy, $G_{\text {tot }}$, in $\mathrm{kJ} . \mathrm{mol}^{-1}$, with respect to the most stable reactant isomer

$\Delta E \ldots$ relative difference in energy, $E(\mathrm{~B} 3 \mathrm{LYP})$, in $\mathrm{kJ} \mathrm{mol}^{-1}$, with respect to the most stable reactant isomer 
Table S2: The molecular energies, contributions to free energy and the difference in the total energies and free energies for $\mathbf{7 b}$. If not explicitly mentioned, the energies are in atomic units (a.u.).

\begin{tabular}{|c|c|c|c|c|c|c|}
\hline $\begin{array}{l}\text { Conformer } \\
\text { (Structure) }\end{array}$ & $\begin{array}{l}E(\text { PBE) } \\
\text { def2-SVP }\end{array}$ & $\begin{array}{l}\text { E(B3LYP) } \\
\text { def2-TZVP }\end{array}$ & $\begin{array}{l}E(P B E+D+ \\
\text { COSMO) } \\
\text { def2-SVP }\end{array}$ & $G_{\text {tot }}$ & $\begin{array}{l}\Delta \boldsymbol{G} \\
\left(\mathrm{kJ} \cdot \mathrm{mol}^{-1}\right)\end{array}$ & $\begin{array}{l}\Delta \boldsymbol{E} \\
\left(\mathrm{kJ} \cdot \mathrm{mol}^{-1}\right)\end{array}$ \\
\hline 2NH-EEEE & -1062.56966 & -1064.40923 & -1062.61547 & -1064.25063 & 25.5 & 19.3 \\
\hline 2NH-EEEZ & -1062.57222 & -1064.41566 & -1062.61606 & -1064.25635 & 10.5 & 2.4 \\
\hline 2NH-EEZE & -1062.57038 & -1064.41490 & -1062.61483 & -1064.25732 & 7.9 & 4.4 \\
\hline 2NH-EEZZ & -1062.57069 & -1064.41471 & -1062.61497 & -1064.25778 & 6.7 & 4.9 \\
\hline 2NH-EZEE & -1062.56928 & -1064.41129 & -1062.61493 & -1064.25165 & 22.8 & 13.9 \\
\hline 2NH-EZEZ & -1062.57216 & -1064.41546 & -1062.61617 & -1064.25567 & 12.3 & 3.0 \\
\hline 2NH-EZZE & -1062.57305 & -1064.41658 & -1062.61744 & -1064.25779 & 6.7 & 0.0 \\
\hline 2NH-EZZZ & -1062.57274 & -1064.41654 & -1062.61838 & -1064.26034 & 0.0 & 0.1 \\
\hline 2NH-ZEEE & -1062.56716 & -1064.40589 & -1062.61644 & -1064.25089 & 24.8 & 28.1 \\
\hline 2NH-ZEEZ & -1062.56959 & -1064.41140 & -1062.61518 & -1064.25165 & 22.8 & 13.6 \\
\hline 2NH-ZEZE & -1062.57396 & -1064.41635 & -1062.61797 & -1064.25616 & 11.0 & 0.6 \\
\hline 2NH-ZEZZ & -1062.57630 & -1064.41496 & -1062.62163 & -1064.25606 & 11.2 & 4.3 \\
\hline 2NH-ZZEE & -1062.56188 & -1064.40501 & -1062.60753 & -1064.24735 & 34.1 & 30.4 \\
\hline 2NH-ZZEZ & -1062.56426 & -1064.40788 & -1062.60869 & -1064.24914 & 29.4 & 22.9 \\
\hline 2NH-ZZZE & -1062.56398 & -1064.40750 & -1062.60993 & -1064.24862 & 30.8 & 23.8 \\
\hline 2NH-ZZZZ & -1062.56380 & -1064.40743 & -1062.60998 & -1064.25472 & 14.7 & 24.0 \\
\hline \multicolumn{7}{|l|}{ enol-form } \\
\hline 3OH-EEEE & -1062.53875 & -1064.38545 & -1062.58516 & -1064.22766 & 85.8 & 81.7 \\
\hline 3OH-EEZE & -1062.55983 & -1064.40333 & -1062.60157 & -1064.24064 & 51.7 & 34.8 \\
\hline 3OH-EZZE & -1062.55997 & -1064.40410 & -1062.60174 & -1064.24186 & 48.5 & 32.8 \\
\hline 3OH-ZEZZ & -1062.55519 & -1064.39868 & -1062.59446 & -1064.23455 & 67.7 & 47.0 \\
\hline 3OH-ZZZZ & -1062.55894 & -1064.40408 & -1062.60003 & -1064.24096 & 50.9 & 32.8 \\
\hline
\end{tabular}

Transition State Corresponding to the Direct $2 \mathrm{NH} \rightarrow$ Phenol Hydrogen Transfer

$\begin{array}{llllll}2 \mathrm{NH}-E Z 7 Z & -106251933 & -1064.35661 & -106255909 & -1064.19874 & \mathbf{1 6 1 7}\end{array}$

\begin{tabular}{|c|c|c|c|c|c|c|}
\hline 2NH-EZZZ & -1062.51933 & -1064.35661 & -1062.55909 & -1064.19874 & 161.7 & 157.5 \\
\hline \multicolumn{7}{|c|}{ 6-Membered Ring Transition States Corresponding to the $3 \mathrm{OH} \rightarrow$ Phenol Hydrogen Transfer } \\
\hline EEZE & -1062.55248 & -1064.39108 & -1062.59292 & -1064.23291 & 72.0 & 67.0 \\
\hline EZZE & -1062.55124 & -1064.38978 & -1062.59181 & -1064.23270 & 72.6 & 70.4 \\
\hline \multicolumn{7}{|c|}{ Product Complex } \\
\hline EEZE & -1062.57157 & -1064.42019 & -1062.61069 & -1064.26245 & -5.6 & -9.5 \\
\hline EZZE & -1062.57067 & -1064.41848 & -1062.60958 & -1064.25993 & 1.1 & -5.0 \\
\hline
\end{tabular}

$E(\mathrm{PBE}) \ldots$ in vacuo energy of the system optimised at the RI-PBE/def2-SVP level of theory

$E$ (B3LYP) in vacuo energy of the system calculated at the B3LYP/def2-TZVP//RI-PBE/def2-SVP

level

$E(\mathrm{PBE}+\mathrm{D}+\mathrm{COSMO})$ energy of the system optimised at the RI-PBE+D+COSMO/def2-SVP level, i.e.

with the empirical dispersion correction and COSMO solvation model

$G_{\mathrm{tot}}=E(\mathrm{~B} 3 \mathrm{LYP})+E(\mathrm{PBE}+\mathrm{D}+\mathrm{COSMO})-E(\mathrm{PBE})+E_{\mathrm{ZPE}}-R T \ln \left(q_{\mathrm{trans}} q_{\mathrm{rot}} q_{\mathrm{vib}}\right)$

$\Delta G$...relative difference in free energy, $G_{\mathrm{tot}}$, in $\mathrm{kJ} \cdot \mathrm{mol}^{-1}$, with respect to the most stable reactant isomer 
$\Delta E \ldots$ relative difference in energy, $E(\mathrm{~B} 3 \mathrm{LYP})$, in $\mathrm{kJ} \mathrm{mol}^{-1}$, with respect to the most stable reactant isomer 
Table S3: The molecular energies, contributions to free energy and the difference in the total energies and free energies for 7c. If not explicitly mentioned, the energies are in atomic units (a.u.).

\begin{tabular}{|c|c|c|c|c|c|c|}
\hline $\begin{array}{l}\text { Conformer } \\
\text { (Structure) }\end{array}$ & $\begin{array}{l}E(P B E) \\
\text { def2-SVP }\end{array}$ & $\begin{array}{l}E(B 3 L Y P) \\
\text { def2-TZVP }\end{array}$ & $\begin{array}{l}E(\mathrm{PBE}+\mathrm{D}+ \\
\text { COSMO) } \\
\text { def2-SVP }\end{array}$ & $G_{\text {tot }}$ & $\begin{array}{l}\Delta \boldsymbol{G} \\
\left(\mathrm{kJ}^{\prime} \mathrm{mol}^{-1}\right)\end{array}$ & $\begin{array}{l}\Delta \boldsymbol{E} \\
\left(\mathrm{kJ} \cdot \mathrm{mol}^{-1}\right)\end{array}$ \\
\hline 2NH-EEEE & -1023.34634 & -1025.11777 & -1023.38824 & -1024.98412 & 22.3 & 36.7 \\
\hline 2NH-EEEZ & -1023.35105 & -1025.12400 & -1023.39024 & -1024.98631 & 16.6 & 20.4 \\
\hline 2NH-EEZE & -1023.34810 & -1025.12098 & -1023.38817 & -1024.98327 & 24.5 & 28.3 \\
\hline 2NH-EEZZ & -1023.34654 & -1025.11967 & -1023.38900 & -1024.98542 & 18.9 & 31.8 \\
\hline 2NH-EZEE & -1023.35435 & -1025.12678 & -1023.39323 & -1024.98914 & 9.1 & 13.1 \\
\hline 2NH-EZEZ & -1023.35955 & -1025.13177 & -1023.39767 & -1024.99262 & $\mathbf{0 . 0}$ & 0.0 \\
\hline 2NH-EZZE & -1023.35407 & -1025.12635 & -1023.39280 & -1024.98573 & 18.1 & 14.2 \\
\hline 2NH-EZZZ & -1023.35299 & -1025.12545 & -1023.39400 & -1024.99192 & 1.8 & 16.6 \\
\hline 2NH-ZEEE & -1023.34758 & -1025.11667 & -1023.39244 & -1024.98249 & 26.6 & 39.6 \\
\hline 2NH-ZEEZ & -1023.34947 & -1025.12080 & -1023.39060 & -1024.98344 & 24.1 & 28.8 \\
\hline 2NH-ZEZE & -1023.35427 & -1025.12511 & -1023.39308 & -1024.98410 & 22.4 & $\mathbf{1 7 . 5}$ \\
\hline 2NH-ZEZZ & -1023.35305 & -1025.12357 & -1023.39446 & -1024.98899 & 9.5 & 21.5 \\
\hline 2NH-ZZEE & -1023.34839 & -1025.12053 & -1023.38841 & -1024.98335 & 24.3 & 29.5 \\
\hline 2NH-ZZEZ & -1023.35428 & -1025.12638 & -1023.39326 & -1024.98748 & 13.5 & 14.1 \\
\hline 2NH-ZZZE & -1023.34802 & -1025.12012 & -1023.38805 & -1024.98125 & 29.8 & 30.6 \\
\hline 2NH-ZZZZ & -1023.34686 & -1025.11908 & -1023.38920 & -1024.98549 & 18.7 & 33.3 \\
\hline \multicolumn{7}{|l|}{ enol-form } \\
\hline 3OH-EEZE & -1023.33490 & -1025.10896 & -1023.37308 & -1024.97026 & $\mathbf{5 8 . 7}$ & 59.9 \\
\hline 3OH-EZZE & -1023.33313 & -1025.10760 & -1023.37198 & -1024.97013 & $\mathbf{5 9 . 0}$ & 63.5 \\
\hline \multicolumn{7}{|c|}{ Transition State Corresponding to the Direct $2 \mathrm{NH} \rightarrow$ Phenol Hydrogen Transfer } \\
\hline 2NH-EZZZ & -1023.28790 & -1025.05495 & -1023.32605 & -1024.92399 & 180.2 & 201.7 \\
\hline \multicolumn{7}{|c|}{ 6-Membered Ring Transition States Corresponding to the $3 \mathrm{OH} \rightarrow$ Phenol Hydrogen Transfer } \\
\hline EEZE & -1023.32768 & -1025.09600 & -1023.36479 & -1024.95812 & 90.6 & 93.9 \\
\hline EZZE & -1023.32688 & -1025.09529 & -1023.36419 & -1024.95895 & 88.4 & 95.8 \\
\hline \multicolumn{7}{|c|}{ Product Complex } \\
\hline EEZE & -1023.34439 & -1025.12327 & -1023.38243 & -1024.99025 & 6.2 & 22.3 \\
\hline EZZE & -1023.34512 & -1025.12350 & -1023.38253 & -1024.98924 & 8.9 & 21.7 \\
\hline
\end{tabular}

$E(\mathrm{PBE}) \ldots$ in vacuo energy of the system optimised at the RI-PBE/def2-SVP level of theory

E(B3LYP) in vacuo energy of the system calculated at the B3LYP/def2-TZVP//RI-PBE/def2-SVP level

$E(\mathrm{PBE}+\mathrm{D}+\mathrm{COSMO})$ energy of the system optimised at the RI-PBE+D+COSMO/def2-SVP level, i.e. with the empirical dispersion correction and COSMO solvation model

$G_{\mathrm{tot}}=E(\mathrm{~B} 3 \mathrm{LYP})+E(\mathrm{PBE}+\mathrm{D}+\mathrm{COSMO})-E(\mathrm{PBE})+E_{\mathrm{ZPE}}-R T \ln \left(q_{\mathrm{trans}} q_{\mathrm{rot}} q_{\mathrm{vib}}\right)$

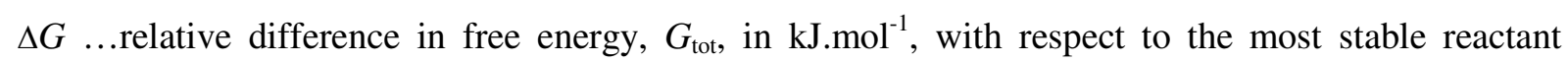
isomer

$\Delta E \ldots$ relative difference in energy, $E(\mathrm{~B} 3 \mathrm{LYP})$, in $\mathrm{kJ} \mathrm{mol}^{-1}$, with respect to the most stable reactant isomer 
Table S4: The molecular energies, contributions to free energy and the difference in the total energies and free energies for 7d. If not explicitly mentioned, the energies are in atomic units (a.u.).

\begin{tabular}{|c|c|c|c|c|c|c|}
\hline $\begin{array}{l}\text { Conformer } \\
\text { (Structure) }\end{array}$ & $\begin{array}{l}E(P B E) \\
\text { def2-SVP }\end{array}$ & $\begin{array}{l}E(B 3 L Y P) \\
\text { def2-TZVP }\end{array}$ & $\begin{array}{l}E(\text { PBE+D+ } \\
\text { COSMO) } \\
\text { def2-SVP }\end{array}$ & $G_{\text {tot }}$ & $\begin{array}{l}\Delta \boldsymbol{G} \\
\left(\mathrm{kJ} \cdot \mathrm{mol}^{-1}\right)\end{array}$ & $\begin{array}{l}\Delta \boldsymbol{E} \\
\left(\mathrm{kJ} \cdot \mathrm{mol}^{-1}\right)\end{array}$ \\
\hline 2NH-EEEE & -1062.57643 & -1064.42032 & -1062.62136 & -1064.26353 & 10.3 & 15.1 \\
\hline 2NH-EEEZ & -1062.58007 & -1064.42532 & -1062.62243 & -1064.26552 & 5.1 & 2.0 \\
\hline 2NH-EEZE & -1062.57830 & -1064.42343 & -1062.62089 & -1064.26487 & 6.8 & 7.0 \\
\hline 2NH-EEZZ & -1062.57689 & -1064.42216 & -1062.62166 & -1064.26669 & 2.0 & 10.3 \\
\hline 2NH-EZEE & -1062.57661 & -1064.42119 & -1062.62130 & -1064.26432 & 8.2 & 12.9 \\
\hline 2NH-EZEZ & -1062.57971 & -1064.42467 & -1062.62251 & -1064.26440 & 8.0 & 3.7 \\
\hline 2NH-EZZE & -1062.58196 & -1064.42608 & -1062.62363 & -1064.26583 & 4.3 & $\mathbf{0 . 0}$ \\
\hline 2NH-EZZZ & -1062.58096 & -1064.42538 & -1062.62501 & -1064.26745 & 0.0 & 1.9 \\
\hline 2NH-ZEEE & -1062.57539 & -1064.41702 & -1062.62309 & -1064.26060 & 18.0 & 23.8 \\
\hline 2NH-ZEEZ & -1062.57723 & -1064.42103 & -1062.62142 & -1064.26111 & 16.7 & 13.3 \\
\hline 2NH-ZEZE & -1062.58282 & -1064.42597 & -1062.62404 & -1064.26226 & 13.6 & 0.3 \\
\hline 2NH-ZEZZ & -1062.58186 & -1064.42456 & -1062.62535 & -1064.26559 & 4.9 & 4.0 \\
\hline 2NH-ZZEE & -1062.56818 & -1064.41336 & -1062.61317 & -1064.25730 & 26.7 & 33.4 \\
\hline 2NH-ZZEZ & -1062.57188 & -1064.41712 & -1062.61262 & -1064.25591 & 30.3 & 23.5 \\
\hline 2NH-ZZZE & -1062.57301 & -1064.41712 & -1062.61617 & -1064.25641 & 29.0 & 23.5 \\
\hline 2NH-ZZZZ & -1062.57196 & -1064.41623 & -1062.61758 & -1064.25854 & 23.4 & 25.9 \\
\hline \multicolumn{7}{|l|}{ enol-form } \\
\hline 3OH-EEZE & -1062.56408 & -1064.4 & -1062.60503 & -1064.24885 & 48.8 & 42.6 \\
\hline 3OH-EZZE & -1062.56274 & -1064.40893 & -1062.60402 & -1064.24928 & 47.7 & 45.0 \\
\hline \multicolumn{7}{|c|}{ Transition State Corresponding to the Direct $2 \mathrm{NH} \rightarrow$ Phenol Hydrogen Transfer } \\
\hline 2NH-EZZZ & -1062.51848 & -1064.35806 & -1062.55993 & -1064.20568 & 162.2 & 178.6 \\
\hline \multicolumn{7}{|c|}{ 6-Membered Ring Transition States Corresponding to the $3 \mathrm{OH} \rightarrow$ Phenol Hydrogen Transfer } \\
\hline EEZE & -1062.55750 & -1064.39779 & -1062.59750 & -1064.23883 & 75.1 & 74.3 \\
\hline EZZE & -1062.55624 & -1064.39655 & -1062.59623 & -1064.23732 & 79.1 & 77.5 \\
\hline \multicolumn{7}{|c|}{ Product Complex } \\
\hline EEZE & -1062.57492 & -1064.42598 & -1062.61566 & -1064.27015 & -7.1 & 0.3 \\
\hline EZZE & -1062.57407 & -1064.42449 & -1062.61441 & -1064.26897 & -4.0 & 4.2 \\
\hline
\end{tabular}

$E(\mathrm{PBE}) \ldots$ in vacuo energy of the system optimised at the RI-PBE/def2-SVP level of theory

$E(\mathrm{~B} 3 \mathrm{LYP})$ in vacuo energy of the system calculated at the B3LYP/def2-TZVP//RI-PBE/def2-SVP level

$E(\mathrm{PBE}+\mathrm{D}+\mathrm{COSMO})$ energy of the system optimised at the RI-PBE+D+COSMO/def2-SVP level, i.e. with the empirical dispersion correction and COSMO solvation model

$G_{\mathrm{tot}}=E(\mathrm{~B} 3 \mathrm{LYP})+E(\mathrm{PBE}+\mathrm{D}+\mathrm{COSMO})-E(\mathrm{PBE})+E_{\mathrm{ZPE}}-R T \ln \left(q_{\mathrm{trans}} q_{\mathrm{rot}} q_{\mathrm{vib}}\right)$

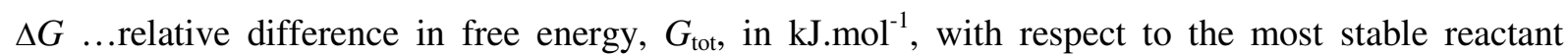
isomer

$\Delta E \ldots$ relative difference in energy, $E(\mathrm{~B} 3 \mathrm{LYP})$, in $\mathrm{kJ}^{\mathrm{mol}}{ }^{-1}$, with respect to the most stable reactant isomer 
Table S5: The molecular energies, contributions to free energy and the difference in the total energies and free energies for decomposition products. If not explicitly mentioned, the energies are in atomic units (a.u.).

\begin{tabular}{|c|c|c|c|c|c|c|}
\hline $\begin{array}{l}\text { Conformer } \\
\text { (Structure) }\end{array}$ & $\begin{array}{l}E(\mathrm{PBE}) \\
\text { def2-SVP }\end{array}$ & $\begin{array}{l}E(B 3 L Y P) \\
\text { def2-TZVP }\end{array}$ & $\begin{array}{l}E(P B E+D+ \\
\text { COSMO) } \\
\text { def2-SVP }\end{array}$ & $G_{\text {tot }}$ & $\begin{array}{l}\Delta \boldsymbol{G} \\
\left(\mathrm{kJ} \cdot \mathrm{mol}^{-1}\right)\end{array}$ & $\begin{array}{l}\Delta \boldsymbol{E} \\
\left(\mathrm{kJ} \cdot \mathrm{mol}^{-1}\right)\end{array}$ \\
\hline$o$-nitrophenol & -511.04060 & -511.91856 & -511.05604 & -511.86260 & 3.6 & 0.0 \\
\hline$p$-nitrophenol & -511.02999 & -511.91394 & -511.05044 & -511.86398 & $\mathbf{0 . 0}$ & 12.1 \\
\hline 3a_EE & -512.29253 & -513.19701 & -512.31001 & -513.13040 & $\mathbf{0 . 0}$ & 0.5 \\
\hline 3a_EZ & -512.29318 & -513.19721 & -512.31015 & -513.13012 & 0.7 & 0.0 \\
\hline 3a_ZE & -512.28746 & -513.19092 & -512.30576 & -513.12593 & 11.7 & 16.5 \\
\hline 3a_ZZ & -512.28804 & -513.19188 & -512.30609 & -513.12683 & 9.4 & 14.0 \\
\hline 3b_EE & -551.52298 & -552.49977 & -551.54306 & -552.41139 & $\mathbf{0 . 0}$ & 0.0 \\
\hline 3b_EZ & -551.52206 & -552.49797 & -551.54197 & -552.40922 & 5.7 & 4.7 \\
\hline 3b_ZE & -551.51556 & -552.49127 & -551.53647 & -552.40350 & 20.7 & 22.3 \\
\hline 3b_ZZ & -551.51439 & -552.49019 & -551.53539 & -552.40281 & 22.5 & 25.2 \\
\hline
\end{tabular}

$E(\mathrm{PBE}) \ldots$ in vacuo energy of the system optimised at the RI-PBE/def2-SVP level of theory

E(B3LYP) in vacuo energy of the system calculated at the B3LYP/def2-TZVP//RI-PBE/def2-SVP level

$E(\mathrm{PBE}+\mathrm{D}+\mathrm{COSMO})$ energy of the system optimised at the RI-PBE+D+COSMO/def2-SVP level, i.e. with the empirical dispersion correction and COSMO solvation model $G_{\mathrm{tot}}=E(\mathrm{~B} 3 \mathrm{LYP})+E(\mathrm{PBE}+\mathrm{D}+\mathrm{COSMO})-E(\mathrm{PBE})+E_{\mathrm{ZPE}}-R T \ln \left(q_{\mathrm{trans}} q_{\mathrm{rot}} q_{\mathrm{vib}}\right)$

$\Delta G$...relative difference in free energy, $G_{\mathrm{tot}}$, in $\mathrm{kJ} . \mathrm{mol}^{-1}$, with respect to the most stable reactant isomer

$\Delta E \ldots$ relative difference in energy, $E(\mathrm{~B} 3 \mathrm{LYP})$, in $\mathrm{kJ} \mathrm{mol}^{-1}$, with respect to the most stable reactant isomer 
Molecular energies (in a.u.) and molecular structures of 7a (Table S1)

32

$\begin{array}{llll}\text { C } & -1.59810 & -4.21913 & 0.68536\end{array}$

$\begin{array}{llll}\text { C } & -1.61160 & -5.60579 & 0.91284\end{array}$

$\begin{array}{llll}\text { C } & -1.67027 & -6.09885 & 2.21857\end{array}$

C $\quad-1.73205-5.19467 \quad 3.29377$

C $\quad-1.74324 \quad-3.81256 \quad 3.06910$

C $\quad-1.67278-3.29851 \quad 1.75974$

$\begin{array}{lllll}0 & -1.65100 & -1.94928 & 1.50679\end{array}$

$\begin{array}{llll}\text { C } & -2.51916 & -1.11788 & 2.21345\end{array}$

$\begin{array}{lllll}\text { o } & -3.44661 & -1.51794 & 2.88558\end{array}$

n $\quad-1.48882 \quad-3.77234-0.71330$

o $\quad-0.87073-2.72972-0.93174$

$\begin{array}{llll}\mathrm{n} & -2.19300 & 0.21073 & 2.03839\end{array}$

c $\quad \begin{array}{llll}-0.93871 & 0.84320 & 1.62347\end{array}$

$\begin{array}{llll}\text { o } & -0.40516 & 1.59479 & 2.42835\end{array}$

$\begin{array}{llll}\text { c } & -0.48008 & 0.63483 & 0.26169\end{array}$

C $\quad-1.279390 .18584-0.74969$

$\begin{array}{lllll}\text { o } & -0.86554 & 0.13108 & -2.01093\end{array}$

C $\quad-1.80503-0.23296-3.04210$

$\begin{array}{llll}\text { C } & -1.20688 & -1.30458 & -3.93662\end{array}$

h $\quad 0.53263 \quad 1.003940 .03783$

$\begin{array}{lllll}\text { o } & -2.00120 & -4.48607 & -1.57766\end{array}$

$\begin{array}{lllll}\text { h } & -2.67447 & 0.78771 & 2.73832\end{array}$

h $\quad-2.32428 \quad-0.12969-0.55904$

$\begin{array}{llll}\text { h } & -2.75114 & -0.58442 & -2.56980\end{array}$

h $\quad-2.02956 \quad 0.69633-3.61416$

h $\quad-1.89235-1.50262-4.78948$

h $\quad-0.22916-0.97129-4.34611$

h $\quad-1.06044-2.24385-3.36462$

h $\quad-1.57105-6.272990 .03963$

h $\quad-1.66505 \quad-7.18527 \quad 2.39862$

h $\quad-1.77644 \quad-5.56893 \quad 4.32918$

h $\quad-1.81296-3.11455 \quad 3.91406$

\section{NH-EEEZ}

$E(B 3 L Y P /$ def2-TZVP) $=-1025.115668207$

32

$\begin{array}{llll}\text { C } & -3.02370 & -4.45287 & 0.72050\end{array}$

C $\quad-2.41131 \quad-5.40004 \quad 1.54684$

$\begin{array}{llll}\text { C } & -1.29679 & -5.02759 & 2.31889\end{array}$

$\begin{array}{llll}\text { C } & -0.81428 & -3.71332 & 2.27656\end{array}$ 
$\begin{array}{llll}\text { C } & -1.40887 & -2.75191 & 1.44095\end{array}$

$\begin{array}{llll}\text { C } & -2.51561 & -3.14526 & 0.64612\end{array}$

$\begin{array}{lllll}0 & -0.85849 & -1.49945 & 1.44903\end{array}$

C $\quad-1.71890 \quad-0.38699 \quad 1.54644$

$\begin{array}{llll}\mathrm{n} & -0.97584 & 0.73182 & 1.26925\end{array}$

c $\quad-1.36312 \quad 2.12464 \quad 1.37224$

$\begin{array}{llll}\text { C } & -2.68784 & 2.50479 & 0.90080\end{array}$

h $\quad-3.03861 \quad 3.50018 \quad 1.21334$

n $\quad-3.14855-2.23683-0.32460$

$\begin{array}{lllll}0 & -4.34277 & -2.39834 & -0.56785\end{array}$

$\begin{array}{lllll}0 & -2.88575 & -0.48244 & 1.85016\end{array}$

o $\quad-2.42808-1.39006-0.86402$

$\begin{array}{lllll}0 & -0.52197 & 2.90605 & 1.79856\end{array}$

C $\quad-3.41865 \quad 1.75670 \quad 0.02423$

$\begin{array}{lllll}0 & -4.61669 & 2.13997 & -0.41697\end{array}$

$\begin{array}{llll}\text { c } & -5.24048 & 1.31969 & -1.43022\end{array}$

c $\quad-6.74370 \quad 1.50392 \quad-1.34100$

$\begin{array}{llll}\text { h } & 0.04221 & 0.60424 & 1.26900\end{array}$

h $\quad-3.04404 \quad 0.79642 \quad-0.37653$

h $\quad-4.85434 \quad 1.64631-2.42372$

h $\quad-4.94908 \quad 0.25539-1.27753$

$\begin{array}{llll}\text { h } & -7.24302 & 0.91118 & -2.13747\end{array}$

h $\quad-7.12654 \quad 1.16055-0.35616$

h $\quad-7.02143 \quad 2.57173 \quad-1.47326$

$\begin{array}{llll}\text { h } & -3.89987 & -4.70181 & 0.10401\end{array}$

h $\quad-2.80344 \quad-6.42819 \quad 1.58819$

$\begin{array}{llll}\text { h } & -0.80650 & -5.76386 & 2.97597\end{array}$

$\begin{array}{llll}\text { h } & 0.03476 & -3.39317 & 2.89979\end{array}$

2NH-EEZE

$E(B 3 L Y P /$ def2-TZVP $)=-1025.112407482$

32

$\begin{array}{llll}\text { C } & -2.55668 & -4.53433 & 0.03983\end{array}$

$\begin{array}{llll}\text { C } & -2.48542 & -5.31611 & 1.19731\end{array}$

$\begin{array}{llll}\text { C } & -1.90140 & -4.78258 & 2.35961\end{array}$

$\begin{array}{llll}\text { C } & -1.41021 & -3.47091 & 2.36234\end{array}$

$\begin{array}{llll}\text { C } & -1.47194 & -2.67681 & 1.20462\end{array}$

$\begin{array}{llll}\text { c } & -2.03980 & -3.22797 & 0.03014\end{array}$

$\begin{array}{lllll}0 & -0.92700 & -1.42111 & 1.29891\end{array}$

C $\quad-1.69144 \quad-0.34028 \quad 0.93383$

$\begin{array}{llll}\mathrm{n} & -0.94165 & 0.82381 & 0.90282\end{array}$

c $\quad 0.40032 \quad 1.08173 \quad 1.32507$

c $\quad 0.96688 \quad 2.337390 .79408$

$\begin{array}{llll}\text { h } & 1.84214 & 2.72588 & 1.33857\end{array}$

n $\quad-2.06196-2.50874-1.26336$

o $\quad-2.86623-2.89408-2.10929$

$\begin{array}{lllll}0 & -2.87631 & -0.38489 & 0.67929\end{array}$

$\begin{array}{lllll}0 & -1.24558 & -1.60024 & -1.42917\end{array}$

$\begin{array}{llll}0 & 1.01382 & 0.33552 & 2.07001\end{array}$

$\begin{array}{llll}\text { c } & 0.57637 & 2.93682 & -0.36313\end{array}$

$\begin{array}{lllll}\text { o } & 1.16183 & 4.04912 & -0.82892\end{array}$

C $\quad 0.78245 \quad 4.48382-2.14701$ 
2NH-EEZZ

$E(B 3 L Y P / d e f 2-T Z V P)=-1025.112080149$

32

C $\quad-3.11615 \quad-3.08811 \quad 1.17656$

C $\quad-1.85887-2.46460 \quad 1.12131$

c $\quad-0.69949-3.27075 \quad 1.01572$

$\begin{array}{llll}\text { C } & -0.79204 & -4.67148 & 1.01497\end{array}$

$\begin{array}{llll}\text { C } & -2.05095 & -5.28057 & 1.06174\end{array}$

C $\quad-3.20971-4.48666 \quad 1.13654$

$\begin{array}{llll}0 & -1.72550 & -1.10817 & 1.23437\end{array}$

$\begin{array}{llll}\text { C } & -2.14205 & -0.29842 & 0.15095\end{array}$

$\begin{array}{llll}0 & -3.01400 & -0.60099 & -0.62029\end{array}$

n $\quad 0.64662 \quad-2.66997 \quad 0.90170$

$\begin{array}{lllll}0 & 0.74351 & -1.56048 & 0.36749\end{array}$

$\begin{array}{llll}\mathrm{n} & -1.34786 & 0.82511 & 0.19719\end{array}$

$\begin{array}{lllll}\text { c } & -1.46188 & 1.98040 & -0.63734\end{array}$

$\begin{array}{llll}0 & -2.51463 & 2.29453 & -1.16544\end{array}$

$\begin{array}{llll}\text { c } & -0.20614 & 2.73941 & -0.78659\end{array}$

$\begin{array}{llll}\text { C } & 1.03867 & 2.20853 & -0.63359\end{array}$

$\begin{array}{lllll}\text { o } & 2.15206 & 2.92814 & -0.82926\end{array}$

C $\quad 3.40133 \quad 2.21323-0.80639$

c $\quad 4.53164 \quad 3.22466-0.80509$

h $\quad-0.31749 \quad 3.77196-1.15366$

$\begin{array}{llll}\text { o } & 1.59438 & -3.32999 & 1.32404\end{array}$

$\begin{array}{llll}\text { h } & -0.54054 & 0.74936 & 0.82120\end{array}$

h $\quad \begin{array}{llll}1.19695 & 1.14366 & -0.36151\end{array}$

$\begin{array}{lllll}\text { h } & 3.43767 & 1.56419 & 0.10035\end{array}$

h $\quad 3.45546 \quad 1.55193-1.70282$

h $\quad 5.51038 \quad 2.69878-0.80081$

h $\quad 4.48443 \quad 3.86944-1.70863$

h $\quad 4.47845 \quad 3.87695 \quad 0.09284$

h $\quad 0.13748 \quad-5.25713 \quad 0.96663$

h $\quad-2.12903 \quad-6.37901 \quad 1.04104$

h $\quad-4.20266-4.96343 \quad 1.17261$

$\begin{array}{llll}\text { h } & -4.01146 & -2.45534 & 1.25684\end{array}$

\section{NH-EZEE}


$E(B 3 L Y P /$ def2-TZVP $)=-1025.117619883$

32

$\begin{array}{llll}\text { C } & -1.38607 & -3.55984 & 1.31552\end{array}$

$\begin{array}{llll}\text { C } & -2.17226 & -4.63414 & 1.76294\end{array}$

C $\quad-3.51728 \quad-4.436642 .09144$

C $\quad-4.07571 \quad-3.15125 \quad 1.98450$

C $\quad-3.28594 \quad-2.072201 .56676$

C $\quad-1.93623-2.25883 \quad 1.22586$

$\begin{array}{llll}\text { o } & -1.25122 & -1.15208 & 0.78718\end{array}$

$\begin{array}{llll}\text { C } & -0.08146 & -0.79966 & 1.44777\end{array}$

$\begin{array}{lllll}0 & 0.24917 & -1.26219 & 2.51723\end{array}$

$\begin{array}{llll}\text { n } & -0.00036 & -3.86897 & 0.89723\end{array}$

$\begin{array}{lllll}\text { o } & 0.54762 & -3.09191 & 0.11131\end{array}$

$\begin{array}{lllll}\mathrm{n} & 0.59211 & 0.20214 & 0.78106\end{array}$

c $\quad 0.48980 \quad 0.82193 \quad-0.50215$

$\begin{array}{lllll}\text { o } & 1.08379 & 1.88845 & -0.64830\end{array}$

$\begin{array}{lllll}\text { C } & -0.24876 & 0.12310 & -1.55839\end{array}$

$\begin{array}{llll}\text { C } & -0.39332 & 0.75253 & -2.75595\end{array}$

$\begin{array}{lllll}0 & -1.01134 & 0.18795 & -3.79867\end{array}$

$\begin{array}{llll}\text { C } & -1.09959 & 0.97319 & -5.00160\end{array}$

$\begin{array}{llll}\text { c } & -1.77007 & 0.13280 & -6.07171\end{array}$

h $\quad-0.63554 \quad-0.89268-1.40521$

$\begin{array}{llll}\text { o } & 0.50167 & -4.90386 & 1.32718\end{array}$

$\begin{array}{llll}\text { h } & 1.31771 & 0.64268 & 1.35727\end{array}$

h $\quad 0.01502 \quad 1.77376-2.89491$

h $\quad-1.68545 \quad 1.89872-4.79001$

h $\quad-0.07399 \quad 1.27934 \quad-5.31441$

h $\quad-1.85606 \quad 0.71612-7.01339$

h $\quad-1.17993 \quad-0.78538-6.27971$

h $\quad-2.78964 \quad-0.17247 \quad-5.75292$

$\begin{array}{llll}\text { h } & -1.69415 & -5.62174 & 1.83472\end{array}$

$\begin{array}{llll}\text { h } & -4.13001 & -5.28658 & 2.43047\end{array}$

h $\quad \begin{array}{llll}-5.13399 & -2.98205 & 2.24117\end{array}$

h $\quad-3.69406 \quad-1.05215 \quad 1.49977$

2NH-EZEZ

$E(B 3 L Y P /$ def2-TZVP $)=-1025.122726203$

32

$\begin{array}{llll}\text { C } & -1.35059 & -3.18314 & 0.17717\end{array}$

C $\quad-1.40133-4.56878-0.04686$

$\begin{array}{llll}\text { C } & -1.98954 & -5.41770 & 0.89618\end{array}$

$\begin{array}{llll}\text { C } & -2.54371 & -4.87380 & 2.06786\end{array}$

C $\quad-2.52125-3.489492 .28030$

C $\quad-1.92535-2.62555 \quad 1.34540$

$\begin{array}{llll}\text { O } & -1.92056 & -1.28955 & 1.65814\end{array}$

$\begin{array}{llll}\text { c } & -2.43640 & -0.38049 & 0.72715\end{array}$

o $\quad-3.06756-0.71139-0.25100$

n $\quad-0.61569-2.37029-0.81956$

$\begin{array}{lllll}0 & -0.16740 & -1.28289 & -0.45187\end{array}$

$\begin{array}{llll}\mathrm{n} & -2.13595 & 0.88143 & 1.18194\end{array}$

http://mc.manuscriptcentral.com/poc 
32

C $\quad-1.95589 \quad-3.99889 \quad 0.12443$

$\begin{array}{llll}\text { C } & -2.62423 & -5.16419 & 0.53547\end{array}$

$\begin{array}{llll}\text { C } & -2.87697 & -5.39571 & 1.89131\end{array}$

$\begin{array}{llll}\text { C } & -2.46732 & -4.44664 & 2.84407\end{array}$

$\begin{array}{llll}\text { C } & -1.82708 & -3.26984 & 2.43573\end{array}$

C $\quad-1.56283 \quad-3.02720 \quad 1.07684$

$\begin{array}{llll}0 & -0.89472 & -1.86463 & 0.78717\end{array}$

C $\quad-1.42283-1.01399-0.15156$

$\begin{array}{lllll}0 & -2.52833 & -1.12228 & -0.63841\end{array}$

$\begin{array}{lllll}\mathrm{n} & -1.63477 & -3.88587 & -1.31647\end{array}$

o $\quad-0.68883-3.16465-1.63696$

$\begin{array}{lllll}\mathrm{n} & -0.55427 & 0.02387 & -0.45128\end{array}$

$\begin{array}{llll}\text { c } & 0.74744 & 0.33277 & 0.03327\end{array}$

$\begin{array}{llll}0 & 1.30000 & -0.30221 & 0.92045\end{array}$

C $\quad 1.333521 .50292-0.65305$

$\begin{array}{llll}\text { C } & 2.57586 & 1.91420 & -0.28475\end{array}$

$\begin{array}{llll}\text { o } & 3.19540 & 2.95818 & -0.84375\end{array}$

$\begin{array}{llll}\text { C } & 4.52186 & 3.25583 & -0.36668\end{array}$

c $\quad 5.019824 .49532-1.08534$

h $\quad 0.79356 \quad 2.03526-1.45364$

o $\quad-2.30586-4.55654-2.09783$

h $\quad-0.948390 .64305-1.16503$

h $\quad 3.11106 \quad 1.36576 \quad 0.51622$

$\begin{array}{llll}\text { h } & 4.48480 & 3.41623 & 0.73629\end{array}$

h $\quad 5.18385 \quad 2.38097-0.56616$

h $\quad 6.04523 \quad 4.74708-0.73953$

h $\quad 5.05079 \quad 4.32948-2.18348$

h $\quad 4.35727 \quad 5.36349-0.88136$ 


$\begin{array}{llll}\text { h } & -2.92710 & -5.87866 & -0.24334 \\ \text { h } & -3.39063 & -6.31820 & 2.20449 \\ \text { h } & -2.65679 & -4.61776 & 3.91613 \\ \text { h } & -1.51479 & -2.50139 & 3.15902\end{array}$

2NH-EZZZ

$E(B 3 L Y P /$ def2-TZVP $)=-1025.116606729$

32

C $\quad-3.51715 \quad-4.45306 \quad 1.54546$

c $\quad-3.11932 \quad-3.11399 \quad 1.42605$

$\begin{array}{llll}\text { C } & -1.80219 & -2.80127 & 1.04624\end{array}$

c $\quad-0.89680-3.857510 .79107$

$\begin{array}{llll}\text { C } & -1.28715 & -5.19626 & 0.94817\end{array}$

C $\quad-2.60458-5.49670 \quad 1.31104$

$\begin{array}{lllll}0 & -1.34836 & -1.50970 & 0.99279\end{array}$

C $\quad-1.99706 \quad-0.581920 .15766$

$\begin{array}{lllll}\mathrm{n} & -1.14945 & 0.50612 & 0.07277\end{array}$

c $\quad-1.43325 \quad 1.74241-0.57139$

C $\quad-0.27181 \quad 2.65381-0.57963$

h $\quad 0.69297 \quad 2.36515-0.13040$

$\begin{array}{lllll}\mathrm{n} & 0.48980 & -3.59448 & 0.34298\end{array}$

$\begin{array}{lllll}0 & 1.34166 & -4.42705 & 0.65540\end{array}$

$\begin{array}{lllll}0 & -3.07541 & -0.75279 & -0.34795\end{array}$

$\begin{array}{lllll}0 & 0.69766 & -2.58537 & -0.33043\end{array}$

$\begin{array}{llll}0 & -2.52851 & 1.99450 & -1.05091\end{array}$

C $\quad-0.41135 \quad 3.87025-1.17096$

$\begin{array}{lllll}0 & 0.57439 & 4.76968 & -1.23585\end{array}$

C $\quad 0.28300 \quad 6.00008-1.92684$

c $\quad 1.50924 \quad 6.88969-1.85498$

$\begin{array}{llll}\text { h } & -0.21633 & 0.36365 & 0.47011\end{array}$

h $\quad-1.38650 \quad 4.14035-1.62456$

h $\quad 0.01550 \quad 5.76747-2.98405$

h $\quad-0.59810 \quad 6.48673-1.44725$

h $\quad 1.31447 \quad 7.84620-2.38574$

h $\quad 1.76941 \quad 7.12044-0.79977$

h $\quad 2.38353 \quad 6.39641-2.33092$

$\begin{array}{llll}\text { h } & -0.53731 & -5.98072 & 0.77021\end{array}$

h $\quad-2.91925 \quad-6.54706 \quad 1.41490$

h $\quad-4.55520 \quad-4.68245 \quad 1.83604$

$\begin{array}{llll}\text { h } & -3.82175 & -2.29381 & 1.62842\end{array}$

\section{NH-ZEEE}

$E(B 3 L Y P /$ def2-TZVP) $=-1025.105315786$

32

$\begin{array}{llll}\text { C } & -1.43228 & -5.17152 & 0.55322\end{array}$

C $\quad-2.07874 \quad-5.88525 \quad 1.56884$

c $\quad-2.48239 \quad-5.21215 \quad 2.73362$

$\begin{array}{llll}\text { C } & -2.26316 & -3.83493 & 2.88952\end{array}$

http://mc.manuscriptcentral.com/poc 


$$
\begin{array}{rrr}
-1.60976 & -3.10590 & 1.87690 \\
-1.18096 & -3.80299 & 0.72244 \\
-1.34495 & -1.75945 & 1.93339 \\
-2.21975 & -0.87756 & 2.54564 \\
-1.77623 & 0.42353 & 2.41710 \\
-0.76082 & 1.05834 & 1.63333 \\
-0.49520 & 0.59106 & 0.26672 \\
0.46605 & 0.93688 & -0.14034 \\
-0.43671 & -3.10785 & -0.34199 \\
-0.83600 & -3.27222 & -1.50204 \\
-3.20839 & -1.19707 & 3.17252 \\
0.53345 & -2.43473 & -0.01176 \\
-0.20849 & 2.04253 & 2.11128 \\
-1.34757 & -0.01493 & -0.60625 \\
-2.57763 & -0.44564 & -0.28973 \\
-3.36868 & -1.02666 & -1.34942 \\
-4.73399 & -1.36850 & -0.78413 \\
-2.16674 & 1.04659 & 3.13254 \\
-1.02942 & -0.18910 & -1.65285 \\
-3.44895 & -0.29101 & -2.18323 \\
-2.84287 & -1.93355 & -1.72768 \\
-5.35988 & -1.83979 & -1.57209 \\
-4.64412 & -2.07990 & 0.06501 \\
-5.25400 & -0.45725 & -0.41863 \\
-1.10357 & -5.65683 & -0.37809 \\
-2.26289 & -6.96472 & 1.45177 \\
-2.98794 & -5.76467 & 3.54204 \\
-2.60969 & -3.31159 & 3.78834
\end{array}
$$

2NH-ZEEZ

$E(B 3 L Y P /$ def2-TZVP $)=-1025.111030212$

32

C $\quad-2.16635 \quad-5.64060 \quad 1.34480$

C $\quad-2.42240-4.95156 \quad 2.54293$

C $\quad-2.12827-3.58588 \quad 2.64585$

C $\quad-1.55052 \quad-2.88798 \quad 1.57054$

$\begin{array}{llll}\text { C } & -1.27760 & -3.59471 & 0.37409\end{array}$

$\begin{array}{llll}\text { c } & -1.60136 & -4.95687 & 0.26340\end{array}$

$\begin{array}{lllll}0 & -1.26821 & -1.56314 & 1.77801\end{array}$

$\begin{array}{llll}\text { C } & -1.77076 & -0.62002 & 0.86705\end{array}$

$\begin{array}{lllll}0 & -2.60039 & -0.88111 & 0.02883\end{array}$

$\begin{array}{lllll}\text { n } & -0.58667 & -2.97341 & -0.77948\end{array}$

o $\quad 0.10511-1.97742-0.56167$

$\begin{array}{lllll}\mathrm{n} & -1.17225 & 0.58181 & 1.17002\end{array}$

c $\quad-1.29116 \quad 1.85187 \quad 0.53260$

$\begin{array}{lllll}0 & -0.33428 & 2.61357 & 0.61181\end{array}$

c $\quad-2.53579 \quad 2.21342 \quad-0.16216$

c $\quad \begin{array}{llll}-3.81339 & 1.85700 & 0.13847\end{array}$

$\begin{array}{llll}0 & -4.15878 & 1.07306 & 1.16744\end{array}$

C $\quad-5.444620 .43534 \quad 1.08996$

$\begin{array}{llll}\text { C } & -5.72924 & -0.23426 & 2.42104\end{array}$

h $\quad-2.40042 \quad 2.99528 \quad-0.92476$ 


\begin{tabular}{|c|c|c|c|}
\hline & -0.71413 & -3.51919 & -1.87272 \\
\hline & -0.32845 & 0.52075 & 1.75116 \\
\hline & -4.65585 & 2.26036 & -0.46159 \\
\hline & -6.21816 & 1.20351 & 0.84713 \\
\hline & -5.41895 & -0.31173 & 0.26457 \\
\hline & -6.72550 & -0.72566 & 2.39263 \\
\hline & -4.96420 & -1.01036 & 2.63871 \\
\hline & -5.72539 & 0.50725 & 3.24895 \\
\hline & -1.38522 & -5.45704 & -0.6918 \\
\hline & -2.40402 & -6.71207 & 1.25307 \\
\hline & -2.86652 & -5.47832 & 3.40300 \\
\hline & -2.339 & -3.01957 & 3.566 \\
\hline
\end{tabular}

\section{NH-ZEZE}

$E(B 3 L Y P / d e f 2-T Z V P)=-1025.115476938$

32

C $\quad \begin{array}{llll}-2.71638 & -5.30919 & 1.63771\end{array}$

C $\quad-2.47584 \quad-4.483772 .75006$

c $\quad-1.93464 \quad-3.20445 \quad 2.57344$

C $\quad-1.60199-2.73169 \quad 1.29185$

C $\quad-1.82382 \quad-3.578490 .17825$

$\begin{array}{llll}\text { c } & -2.39440 & -4.84984 & 0.35679\end{array}$

$\begin{array}{lllll}0 & -1.04037 & -1.48293 & 1.23375\end{array}$

$\begin{array}{llll}\text { C } & -1.57198 & -0.55253 & 0.37187\end{array}$

$\begin{array}{lllll}0 & -2.62187 & -0.68781 & -0.22184\end{array}$

n $\quad-1.41484-3.21797 \quad-1.19748$

o $\quad-0.52003 \quad-2.38136-1.32844$

$\begin{array}{llll}\mathrm{n} & -0.78897 & 0.58444 & 0.28950\end{array}$

$\begin{array}{llll}\text { c } & 0.43717 & 0.91863 & 0.91746\end{array}$

$\begin{array}{llll}\text { o } & 0.97583 & 0.23050 & 1.77085\end{array}$

$\begin{array}{llll}\text { C } & 1.03228 & 2.19865 & 0.45494\end{array}$

$\begin{array}{lllll}\text { C } & 0.59718 & 3.07065 & -0.49294\end{array}$

$\begin{array}{lllll}0 & -0.55737 & 2.92549 & -1.17977\end{array}$

C $\quad-0.78265 \quad 3.79834-2.30411$

$\begin{array}{lllll}\text { c } & -2.20008 & 3.58781 & -2.80051\end{array}$

$\begin{array}{llll}\text { h } & 1.97451 & 2.44498 & 0.96685\end{array}$

o $\quad-1.96457-3.81163-2.12315$

$\begin{array}{llll}\text { h } & -1.16042 & 1.26699 & -0.38421\end{array}$

$\begin{array}{lllll}\text { h } & 1.18437 & 3.97777 & -0.73833\end{array}$

h $\quad-0.61779 \quad 4.85218-1.97776$

h $\quad-0.03698 \quad 3.55889-3.09721$

h $\quad-2.39175 \quad 4.24258-3.67737$

h $\quad-2.35886 \quad 2.53321 \quad-3.11260$

h $\quad-2.93990 \quad 3.83508-2.00946$

h $\quad-2.56510-5.46196-0.54049$

h $\quad-3.15123 \quad-6.31271 \quad 1.76765$

h $\quad-2.72114 \quad-4.83426 \quad 3.76564$

$\begin{array}{llll}\text { h } & -1.75450 & -2.53057 & 3.42470\end{array}$

2NH-ZEZZ

http://mc.manuscriptcentral.com/poc 
32

$\begin{array}{llll}\text { C } & -3.58463 & -4.36653 & 1.53588 \\ \text { C } & -3.25424 & -3.01155 & 1.38680 \\ \text { C } & -1.92804 & -2.63914 & 1.10551 \\ \text { C } & -0.95001 & -3.65421 & 0.97145\end{array}$

c $\quad-1.27313-5.00572 \quad 1.16464$

$\begin{array}{llll}\text { C } & -2.59900 & -5.36466 & 1.43275\end{array}$

$\begin{array}{llll}0 & -1.52617 & -1.33596 & 1.03828\end{array}$

$\begin{array}{llll}\text { C } & -2.10309 & -0.46491 & 0.08227\end{array}$

$\begin{array}{lllll}\mathrm{n} & -1.19225 & 0.55494 & -0.07439\end{array}$

c $\quad-1.33825 \quad 1.69356-0.90840$

$\begin{array}{llll}\text { C } & -0.09521 & 2.48397 & -1.07985\end{array}$

h $\quad-0.22105 \quad 3.35841 \quad-1.73527$

n $\quad 0.44284 \quad-3.31912 \quad 0.61606$

$\begin{array}{lllll}0 & 1.33309 & -4.02867 & 1.08187\end{array}$

$\begin{array}{lllll}0 & -3.17182 & -0.64697 & -0.43962\end{array}$

$\begin{array}{lllll}0 & 0.62235 & -2.36552 & -0.14719\end{array}$

$\begin{array}{lllll}0 & -2.38966 & 1.99148 & -1.45140\end{array}$

c $\quad 1.14816 \quad 2.25855-0.57404$

$\begin{array}{lllll}0 & 1.44283 & 1.27037 & 0.29559\end{array}$

$\begin{array}{llll}\text { C } & 2.82894 & 0.93611 & 0.52262\end{array}$

$\begin{array}{llll}\text { c } & 3.30779 & -0.15408 & -0.42321\end{array}$

$\begin{array}{llll}\text { h } & -0.26820 & 0.40218 & 0.35325\end{array}$

$\begin{array}{llll}\text { h } & 1.99647 & 2.92139 & -0.83966\end{array}$

$\begin{array}{llll}\text { h } & 3.43569 & 1.86708 & 0.43052\end{array}$

$\begin{array}{llll}\text { h } & 2.87147 & 0.60015 & 1.57972\end{array}$

h $\quad 4.36092-0.42016-0.18311$

$\begin{array}{llll}\text { h } & 2.68027 & -1.06649 & -0.32307\end{array}$

h $\quad 3.27106 \quad 0.18775 \quad-1.48081$

h $\quad-0.47083-5.75402 \quad 1.08583$

h $\quad-2.86403 \quad-6.42563 \quad 1.56359$

h $\quad-4.62954 \quad-4.64524 \quad 1.74802$

h $\quad-4.01355 \quad-2.22425 \quad 1.49019$

2NH-ZZEE

$E(B 3 L Y P /$ def2-TZVP) $=-1025.111662524$

32

C $\quad-2.12067 \quad-4.82793 \quad 1.53832$

C $\quad-3.46675 \quad-4.73485 \quad 1.90682$

C $\quad-4.08927 \quad-3.47515 \quad 1.94678$

$\begin{array}{llll}\text { C } & -3.36195 & -2.31911 & 1.63473\end{array}$

$\begin{array}{llll}\text { c } & -2.01180 & -2.39937 & 1.25449\end{array}$

C $\quad-1.39812 \quad-3.67367 \quad 1.19507$

$\begin{array}{lllll}0 & -1.38857 & -1.22038 & 0.93026\end{array}$

$\begin{array}{llll}\text { c } & -0.22190 & -0.88594 & 1.61125\end{array}$

$\begin{array}{llll}\text { n } & 0.38691 & 0.21448 & 1.05100\end{array}$

c $\quad 0.203150 .98183 \quad-0.15004$

$\begin{array}{lllll}\text { C } & -0.46106 & 0.31660 & -1.27484\end{array}$

h $\quad-0.65268-0.76276-1.21699$ 


\section{NH-ZZEZ}

$E(B 3 L Y P /$ def2-TZVP) $=-1025.117170896$

32

C $\quad-1.72098 \quad-4.90195 \quad 0.17263$

C $\quad-2.41986 \quad-5.55169 \quad 1.19506$

C $\quad-2.87452 \quad-4.81305 \quad 2.30112$

$\begin{array}{llll}\text { c } & -2.64412 & -3.43304 & 2.36881\end{array}$

$\begin{array}{llll}\text { C } & -1.93576 & -2.76856 & 1.35282\end{array}$

$\begin{array}{lllll}\text { c } & -1.46171 & -3.52387 & 0.25271\end{array}$

o $\quad-1.73282-1.422851 .52369$

c $\quad-2.11399-0.550690 .49626$

$\begin{array}{llll}\mathrm{n} & -1.64690 & 0.69714 & 0.82544\end{array}$

$\begin{array}{llll}\text { c } & -1.77434 & 1.96359 & 0.16173\end{array}$

C $\quad-2.52678 \quad 1.97976-1.09343$

h $\quad-2.94474 \quad 1.03493 \quad-1.46778$

n $\quad-0.62274-2.93701-0.81706$

o $\quad-0.56674-3.54183-1.88528$

$\begin{array}{lllll}0 & -2.77948 & -0.89445 & -0.45589\end{array}$

$\begin{array}{lllll}0 & 0.00030 & -1.90732 & -0.55374\end{array}$

$\begin{array}{lllll}0 & -1.23857 & 2.92247 & 0.70480\end{array}$

C $\quad-2.73630 \quad 3.10086-1.84320$

o $\quad-2.309324 .32524-1.53106$

C $\quad-2.56999 \quad 5.37014-2.48383$

c $\quad-2.11991 \quad 6.68631-1.87838$

$\begin{array}{llll}\text { h } & -1.07990 & 0.76476 & 1.67744\end{array}$

h $\quad-3.30796 \quad 3.01636-2.79084$

h $\quad-2.01393 \quad 5.15023-3.42586$

h $\quad-3.65985 \quad 5.38732-2.72299$

h $\quad-2.29799 \quad 7.51563-2.59610$

h $\quad-2.67900 \quad 6.90065-0.94256$

h $\quad \begin{array}{llll}-1.03670 & 6.65451 & -1.63489\end{array}$ 
h $\quad-1.34804 \quad-5.44047 \quad-0.71062$

$\begin{array}{llll}\text { h } & -2.60771 & -6.63488 & 1.12934\end{array}$

h $\quad-3.42623 \quad-5.31207 \quad 3.11414$

h $\quad-3.01396 \quad-2.82952 \quad 3.21182$

2NH-ZZZE

$E(B 3 L Y P /$ def2-TZVP $)=-1025.110324335$

32

C $\quad-2.71091 \quad-5.18300 \quad 0.50399$

C $\quad-2.97961 \quad-5.42361 \quad 1.85515$

$\begin{array}{llll}\text { C } & -2.56016 & -4.49221 & 2.82107\end{array}$

$\begin{array}{llll}\text { C } & -1.89442 & -3.32369 & 2.43031\end{array}$

C $\quad-1.61472 \quad-3.07134 \quad 1.07604$

C $\quad-2.01775 \quad-4.02608 \quad 0.11058$

$\begin{array}{llll}0 & -0.92435 & -1.91822 & 0.80452\end{array}$

c $\quad-1.42878-1.04783-0.13053$

$\begin{array}{llll}\mathrm{n} & -0.54265 & -0.02051 & -0.40712\end{array}$

$\begin{array}{llll}\text { c } & 0.75984 & 0.27002 & 0.10950\end{array}$

c $\quad 1.35843 \quad 1.43903-0.56736$

h $\quad 0.80683 \quad 1.94152-1.37834$

n $\quad-1.68204-3.90480-1.32615$

$\begin{array}{lllll}0 & -2.36079 & -4.55271 & -2.12035\end{array}$

$\begin{array}{llll}0 & -2.53055 & -1.13651 & -0.63117\end{array}$

o $\quad-0.71797-3.20134-1.63107$

$\begin{array}{llll}0 & 1.28084 & -0.37806 & 1.00105\end{array}$

$\begin{array}{llll}\text { C } & 2.58361 & 1.96224 & -0.27362\end{array}$

$\begin{array}{lllll}\text { o } & 3.40592 & 1.49337 & 0.66463\end{array}$

C $\quad 4.652322 .18698 \quad 0.84791$

c $\quad \begin{array}{llll}5.44218 & 1.46279 & 1.92145\end{array}$

h $\quad-0.922280 .61124-1.11732$

h $\quad 2.94910 \quad 2.84201-0.84378$

h $\quad 5.20810 \quad 2.20382-0.11936$

h $\quad 4.44286 \quad 3.24200 \quad 1.14497$

$\begin{array}{llll}\text { h } & 6.41197 & 1.97771 & 2.09221\end{array}$

h $\quad 4.87752 \quad 1.443592 .87779$

$\begin{array}{llll}\text { h } & 5.64599 & 0.41380 & 1.61835\end{array}$

h $\quad-3.01998-5.88392-0.28464$

$\begin{array}{llll}\text { h } & -3.51341 & -6.33927 & 2.15440\end{array}$

h $\quad-2.76218-4.67057 \quad 3.88967$

h $\quad-1.57252 \quad-2.56924 \quad 3.16405$

2NH-ZZZZ

$E(B 3 L Y P /$ def2-TZVP $)=-1025.110214269$

32

$\begin{array}{llll}\text { C } & -2.70285 & -5.32137 & 1.35821\end{array}$

$\begin{array}{llll}\text { C } & -3.55058 & -4.25434 & 1.70431\end{array}$

c $\quad-3.11857 \quad-2.92596 \quad 1.58356$

C $\quad-1.83183-2.64615 \quad 1.08852$

http://mc.manuscriptcentral.com/poc 
C $\quad-0.99290 \quad-3.72576 \quad 0.72240$

$\begin{array}{llll}\text { C } & -1.41504 & -5.05470 & 0.88089\end{array}$

$\begin{array}{llll}0 & -1.33018 & -1.37496 & 1.02465\end{array}$

$\begin{array}{llll}\text { c } & -2.04211 & -0.36346 & 0.34987\end{array}$

$\begin{array}{lllll}0 & -3.19898 & -0.43848 & 0.02638\end{array}$

$\begin{array}{lllll}\mathrm{n} & 0.35498 & -3.49819 & 0.15300\end{array}$

o $\quad 0.53916-2.47739-0.50969$

$\begin{array}{lllll}\mathrm{n} & -1.14317 & 0.66791 & 0.17035\end{array}$

c $\quad-1.44319 \quad 1.96645 \quad-0.34982$

$\begin{array}{lllll}0 & -2.58220 & 2.31776 & -0.60105\end{array}$

$\begin{array}{llll}\text { C } & -0.22287 & 2.77850 & -0.52237\end{array}$

$\begin{array}{lllll}\text { C } & -0.20618 & 4.06039 & -0.98977\end{array}$

$\begin{array}{lllll}0 & -1.28856 & 4.75388 & -1.33977\end{array}$

C $\quad-1.08009 \quad 6.08513 \quad-1.84383$

$\begin{array}{llll}\text { c } & -2.43606 & 6.68828 & -2.15693\end{array}$

h $\quad 0.75955 \quad 2.34425-0.27563$

$\begin{array}{lllll}\text { o } & & 1.19976 & -4.37019 & 0.36006\end{array}$

$\begin{array}{llll}\text { h } & -0.17072 & 0.43645 & 0.39324\end{array}$

h $\quad 0.76456 \quad 4.58820-1.09552$

h $\quad-0.53802 \quad 6.68640 \quad-1.07593$

$\begin{array}{lllll}\text { h } & -0.44170 & 6.03368 & -2.75737\end{array}$

h $\quad \begin{array}{llll}-2.31143 & 7.71942 & -2.55187\end{array}$

h $\quad-2.96956 \quad 6.07913-2.91713$

h $\quad-3.06657 \quad 6.73162 \quad-1.24358$

h $\quad-0.71351 \quad-5.858120 .61314$

$\begin{array}{llll}\text { h } & -3.04431 & -6.36308 & 1.46475\end{array}$

$\begin{array}{llll}\text { h } & -4.56447 & -4.45697 & 2.08599\end{array}$

h $\quad-3.76937 \quad-2.08979 \quad 1.87201$

3OH-EEZE

$E(B 3 L Y P /$ def2-TZVP) $=-1025.102531907$

32

$\begin{array}{llll}\text { C } & 0.36724 & 2.63078 & 3.32665\end{array}$

C $\quad 0.12467 \quad 1.925542 .14191$

$\begin{array}{llll}\text { C } & -0.01561 & 0.52476 & 2.14592\end{array}$

C $\quad 0.08900-0.15140 \quad 3.38933$

C $\quad 0.30169 \quad 0.56050 \quad 4.58349$

$\begin{array}{lllll}\text { C } & 0.44986 & 1.94957 & 4.55523\end{array}$

$\begin{array}{llll}0 & -0.22000 & -0.10065 & 0.95681\end{array}$

$\begin{array}{lllll}\mathrm{n} & 0.02546 & -1.61731 & 3.49557\end{array}$

o $\quad-0.26477-2.10065 \quad 4.58284$

$\begin{array}{lllll}0 & 0.30950 & -2.30353 & 2.49823\end{array}$

$\begin{array}{llll}\text { c } & -1.44820 & -0.87859 & 0.78363\end{array}$

$\begin{array}{lllll}\mathrm{n} & -1.41905 & -1.54987 & -0.38851\end{array}$

C $\quad-0.36406-2.15753-0.88124$

C $\quad-0.35709-2.66986-2.24218$

c $\quad-1.45142-2.54997-3.04362$

o $\quad-1.47303-3.00051-4.30085$

C $\quad-2.71512-2.86424-5.01816$

C $\quad-2.51111-3.38562-6.42791$

$\begin{array}{lllll}0 & -2.33739 & -0.78224 & 1.59667\end{array}$

$\begin{array}{llll}0 & 0.76860 & -2.41830 & -0.22043\end{array}$ 
3OH-EZEE

$E(B 3 L Y P /$ def2-TZVP $)=-1025.102420340$

32

$\begin{array}{llll}\text { C } & -0.91007 & 1.18974 & -4.65526\end{array}$

$\begin{array}{llll}\text { C } & -0.68912 & 1.38881 & -3.28935\end{array}$

$\begin{array}{llll}\text { C } & -0.33850 & 0.31016 & -2.44807\end{array}$

C $\quad-0.20271-0.97864-3.03504$

C $\quad-0.43244-1.16913-4.41152$

C $\quad-0.78514-0.09159-5.22416$

$\begin{array}{lllll}0 & -0.17935 & 0.48464 & -1.11052\end{array}$

n $\quad 0.19037-2.17638-2.26436$

o $\quad 0.42622-3.20344-2.89688$

o $\quad 0.26919-2.10965-1.02998$

C $\quad 0.60452 \quad 1.60757 \quad-0.57223$

$\begin{array}{llll}\mathrm{n} & 0.52606 & 1.65593 & 0.77505\end{array}$

c $\quad 0.24104 \quad 0.65824 \quad 1.58742$

c $\quad 0.100820 .95545 \quad 3.00210$

h $\quad 0.25058 \quad 2.00145 \quad 3.30541$

$\begin{array}{llll}\text { o } & 1.18297 & 2.35428 & -1.32518\end{array}$

$\begin{array}{llll}0 & 0.10142 & -0.63082 & 1.26662\end{array}$

$\begin{array}{llll}\text { C } & -0.19542 & -0.00724 & 3.92047\end{array}$

$\begin{array}{llll}0 & -0.31694 & 0.26552 & 5.22316\end{array}$

c $\quad-0.65951-0.83286 \quad 6.08905$

c $\quad-0.67452-0.326607 .51882$

h $\quad-0.34283 \quad-1.05954 \quad 3.60887$

h $\quad-1.65798 \quad-1.23088 \quad 5.79194$

h $\quad 0.08986-1.64818 \quad 5.95740$

h $\quad-0.94059-1.15424 \quad 8.21062$

$\begin{array}{llll}\text { h } & 0.32293 & 0.06714 & 7.80919\end{array}$

h $\quad-1.42088 \quad 0.48715 \quad 7.64186$

h $\quad-0.31899-2.18611-4.81194$

h $\quad-0.96512 \quad-0.24870-6.29922$

h $\quad-1.18981 \quad 2.04974-5.28526$

h $\quad-0.78750 \quad 2.38877 \quad-2.84662$

$\begin{array}{llll}\text { h } & 0.09395 & -0.77999 & 0.27196\end{array}$

Transition State for EEZE isomer 
$E(B 3 L Y P /$ def2-TZVP) $=-1025.088955179$

32

$\begin{array}{llll}\text { C } & 0.38521 & 1.75400 & 3.44851\end{array}$

C $\quad 0.328420 .720852 .47614$

$\begin{array}{llll}\text { C } & -0.00937 & -0.58626 & 2.95115\end{array}$

$\begin{array}{llll}\text { c } & -0.24257 & -0.82838 & 4.31938\end{array}$

c $\quad-0.17032 \quad 0.21090 \quad 5.24699$

C $\quad 0.14430 \quad 1.51106 \quad 4.80152$

$\begin{array}{lllll}0 & 0.59367 & 1.04594 & 1.21011\end{array}$

n $\quad-0.13404-1.740822 .04885$

$\begin{array}{llll}\text { o } & -0.13251 & -1.53510 & 0.82686\end{array}$

$\begin{array}{llll}0 & -0.24200 & -2.86041 & 2.55070\end{array}$

$\begin{array}{lllll}\text { C } & -0.92205 & 0.98341 & -0.00248\end{array}$

$\begin{array}{lllll}0 & -1.89049 & 1.23919 & 0.62349\end{array}$

n $\quad-0.44230 \quad 0.75907-1.17572$

c $\quad 0.78601 \quad 0.30053-1.51197$

$\begin{array}{lllll}0 & 1.73837 & 0.09208 & -0.66421\end{array}$

$\begin{array}{llll}\text { C } & 1.06386 & 0.02557 & -2.90379\end{array}$

$\begin{array}{llll}\text { c } & 0.10733 & 0.18407 & -3.86546\end{array}$

o $\quad 0.33711-0.08609-5.14644$

C $\quad-0.72434 \quad 0.19014-6.08659$

$\begin{array}{llll}\text { C } & -0.40301 & -0.51020 & -7.39225\end{array}$

h $\quad 2.07302 \quad-0.33149-3.15691$

$\begin{array}{lllll}\text { h } & -0.90482 & 0.54084 & -3.58787\end{array}$

h $\quad-1.68957-0.16660-5.65962$

h $\quad-0.79159 \quad 1.29400 \quad-6.22484$

h $\quad-1.19503 \quad-0.29216-8.14034$

h $\quad 0.56874 \quad-0.15927-7.80016$

h $\quad-0.34731 \quad-1.61054-7.24972$

h $\quad-0.48505 \quad-1.85774 \quad 4.61929$

$\begin{array}{llll}\text { h } & -0.36227 & 0.01314 & 6.31339\end{array}$

h $\quad 0.19983 \quad 2.34360 \quad 5.52245$

h $\quad 0.63469 \quad 2.76096 \quad 3.07991$

$\begin{array}{llll}\text { h } & 1.34357 & 0.38676 & 0.33426\end{array}$

Transition State for EZZE isomer

$E(B 3 L Y P /$ def2-TZVP)=-1025.088679583

32

C $\quad-2.85546 \quad-2.39361 \quad 1.40905$

$\begin{array}{llll}\text { c } & -2.46863 & -2.86977 & 0.15465\end{array}$

$\begin{array}{llll}\text { c } & -1.87536 & -4.14756 & -0.01231\end{array}$

$\begin{array}{llll}\text { C } & -1.70592 & -4.93471 & 1.16918\end{array}$

C $\quad-2.08198 \quad-4.444812 .43475$

$\begin{array}{llll}\text { C } & -2.66111 & -3.18149 & 2.56168\end{array}$

o $\quad-1.51555-4.51543-1.24471$

$\begin{array}{lllll}\text { n } & -1.13524 & -6.28940 & 1.12863\end{array}$

$\begin{array}{llll}0 & -0.75109 & -6.78684 & 2.18810\end{array}$

$\begin{array}{lllll}0 & -1.07787 & -6.86901 & 0.03545\end{array}$

C $\quad-2.65161-5.81376-2.06579$

$\begin{array}{lllll}\text { n } & -1.93778 & -6.44794 & -2.93751\end{array}$

http://mc.manuscriptcentral.com/poc 


$$
\begin{array}{ccc}
-0.59582 & -6.47822 & -3.05223 \\
-0.05104 & -7.35208 & -4.06751 \\
-0.75797 & -7.92356 & -4.68607 \\
-3.74131 & -5.68103 & -1.62925 \\
0.21640 & -5.78352 & -2.31364 \\
1.29824 & -7.47767 & -4.23517 \\
1.82737 & -8.27708 & -5.15751 \\
3.26823 & -8.36906 & -5.20306 \\
3.65385 & -9.21519 & -6.40049 \\
1.99748 & -6.90538 & -3.59364 \\
3.69343 & -7.34150 & -5.27716 \\
3.62570 & -8.82599 & -4.25152 \\
4.75945 & -9.30980 & -6.45357 \\
3.21862 & -10.23406 & -6.32093 \\
3.29487 & -8.75313 & -7.34486 \\
-1.90809 & -5.09378 & 3.30489 \\
-2.96480 & -2.81092 & 3.55337 \\
-3.31583 & -1.39525 & 1.49370 \\
-2.60647 & -2.26789 & -0.75680 \\
-0.41406 & -5.18058 & -1.63965
\end{array}
$$

\section{Product Complex EEZE}

$$
E(B 3 L Y P / \text { def2-TZVP) }=-1025.117420818
$$

32

C $\quad 0.27589 \quad 1.59575 \quad 5.12820$

C $\quad 0.83664 \quad 1.74549 \quad 3.86210$

$\begin{array}{llll}\text { C } & 0.73556 & 0.72174 & 2.88443\end{array}$

c $\quad 0.03141-0.464923 .25896$

c $\quad-0.53303 \quad-0.60853 \quad 4.54450$

$\begin{array}{llll}\text { C } & -0.41496 & 0.41351 & 5.48168\end{array}$

$\begin{array}{lllll}\text { o } & 1.30145 & 0.94782 & 1.70042\end{array}$

$\begin{array}{llll}\mathrm{n} & -0.12915 & -1.56407 & 2.32144\end{array}$

$\begin{array}{llll}\text { o } & -0.75141 & -2.56460 & 2.66415\end{array}$

$\begin{array}{llll}0 & 0.38565 & -1.43442 & 1.18535\end{array}$

$\begin{array}{lllll}\text { c } & -1.23381 & 0.50850 & -0.43508\end{array}$

$\begin{array}{lllll}\text { n } & -0.62256 & 0.37137 & -1.48848\end{array}$

c $\quad 0.74871 \quad 0.13945-1.83246$

c $\quad 0.98630-0.01151-3.26334$

$\begin{array}{lllll}\text { C } & -0.00751 & 0.05188 & -4.19338\end{array}$

$\begin{array}{llll}0 & 0.22610 & -0.08908 & -5.50049\end{array}$

c $\quad-0.91194-0.00717-6.38119$

$\begin{array}{llll}\text { C } & -0.42577 & -0.19387 & -7.80552\end{array}$

$\begin{array}{lllll}0 & -1.93498 & 0.66370 & 0.49867\end{array}$

$\begin{array}{lllll}0 & 1.62841 & 0.08242 & -0.98262\end{array}$

h $\quad 2.02982-0.18594-3.56562$

h $\quad-1.05762 \quad 0.22497-3.88403$

h $\quad-1.64639-0.79701-6.09878$

h $\quad-1.40241 \quad 0.98563 \quad-6.24883$

h $\quad-1.28424 \quad-0.13555-8.50845$

$\begin{array}{llll}\text { h } & 0.30700 & 0.59483 & -8.07951\end{array}$

h $\quad 0.06296-1.18387 \quad-7.92947$

h $\quad-1.06125-1.54702 \quad 4.76526$ 


$\begin{array}{lrrr}\mathrm{h} & -0.85588 & 0.30040 & 6.48419 \\ \mathrm{~h} & 0.37590 & 2.41309 & 5.86143 \\ \mathrm{~h} & 1.37794 & 2.65953 & 3.57345 \\ \mathrm{~h} & 1.19380 & 0.14208 & 1.10200\end{array}$

Product Complex EEZE

$E(B 3 L Y P /$ def2-TZVP $)=-1025.117669801$

32

$\begin{array}{llll}\text { c } & -3.07854 & -2.97735 & 2.60245\end{array}$

C $\quad-2.71968$-2.06282 1.58522

$\begin{array}{llll}\text { C } & -1.98647 & -2.47764 & 0.47582\end{array}$

$\begin{array}{llll}\text { C } & -1.57470 & -3.82753 & 0.32677\end{array}$

$\begin{array}{llll}\text { C } & -1.94693 & -4.73768 & 1.36473\end{array}$

$\begin{array}{llll}\text { C } & -2.69042 & -4.30879 & 2.48494\end{array}$

$\begin{array}{lllll}0 & -0.87708 & -4.13907 & -0.76448\end{array}$

$\begin{array}{llll}\text { n } & -1.57044 & -6.14041 & 1.29728\end{array}$

$\begin{array}{llll}0 & -0.90118 & -6.51243 & 0.30434\end{array}$

$\begin{array}{lllll}0 & -1.91103 & -6.90049 & 2.19836\end{array}$

C $\quad-2.70673-6.59365-2.12545$

o $\quad-3.59772-6.22576-1.44860$

$\mathrm{n} \quad-1.88266-7.04951-2.90900$

c $\quad-0.47679-6.93428-3.09216$

$\begin{array}{lllll}\text { o } & 0.21377 & -6.19860 & -2.39198\end{array}$

c $\quad 0.01511-7.77063-4.18564$

C $\quad 1.34589-7.76531-4.47815$

o $\quad 1.87461 \quad-8.49846-5.45883$

C $\quad 3.29936-8.39413-5.65605$

C $\quad 3.68722 \quad-9.29384-6.81348$

$\begin{array}{llll}\text { h } & -0.69310 & -8.39198 & -4.75432\end{array}$

h $\quad 2.03374-7.12946-3.88492$

h $\quad 3.55831-7.33028-5.86631$

h $\quad 3.81748-8.69763-4.71674$

h $\quad 4.78249-9.23209-6.98939$

h $\quad 3.42561-10.35172-6.59702$

h $\quad 3.16446 \quad-8.98752 \quad-7.74475$

h $\quad-2.94452 \quad-5.06208 \quad 3.24414$

h $\quad-3.65890-2.64396 \quad 3.47659$

h $\quad-3.02153 \quad-1.00550 \quad 1.66670$

h $\quad-1.70046-1.77663-0.32335$

h $\quad-0.61798 \quad-5.11498-0.74572$

Molecular energies (in a.u.) and molecular structures of $7 \mathrm{~b}$ (Table S2)

2NH-EEEE

$E(B 3 L Y P /$ def2-TZVP) $=-1064.409226655$

35

C $\quad-1.55348 \quad-5.59376 \quad 0.90097$

http://mc.manuscriptcentral.com/poc 
$\begin{array}{llll}\text { C } & -1.67437 & -6.09667 & 2.19913\end{array}$

$\begin{array}{llll}\text { C } & -1.78244 & -5.20137 & 3.27798\end{array}$

C $\quad-1.77824 \quad-3.81695 \quad 3.06534$

C $\quad-1.64399-3.29418 \quad 1.76493$

$\begin{array}{llll}\text { C } & -1.52240 & -4.20566 & 0.68812\end{array}$

$\begin{array}{lllll}0 & -1.60800 & -1.94390 & 1.51757\end{array}$

C $\quad-2.51873-1.11407 \quad 2.17039$

$\begin{array}{llll}\mathrm{n} & -2.20627 & 0.21538 & 1.97690\end{array}$

$\begin{array}{llll}\text { c } & -0.93198 & 0.83529 & 1.60776\end{array}$

$\begin{array}{llll}\text { c } & -0.42562 & 0.64635 & 0.25153\end{array}$

$\begin{array}{llll}\text { c } & 0.98443 & 1.09877 & -0.01927\end{array}$

n $\quad-1.34589-3.74130-0.69782$

o $\quad-1.87398-4.41133-1.58844$

$\begin{array}{lllll}0 & -3.46555 & -1.51551 & 2.81411\end{array}$

$\begin{array}{lllll}0 & -0.66428 & -2.73250 & -0.87801\end{array}$

$\begin{array}{lllll}0 & -0.40349 & 1.55810 & 2.44402\end{array}$

$\begin{array}{llll}\text { C } & -1.26672 & 0.20798 & -0.73662\end{array}$

$\begin{array}{lllll}0 & -0.88661 & 0.11837 & -2.01074\end{array}$

C $\quad-1.85987-0.24559-3.00764$

C $\quad-1.29696-1.33220-3.90762$

$\begin{array}{llll}\text { h } & -2.71462 & 0.79888 & 2.65213\end{array}$

h $\quad \begin{array}{llll}1.22570 & 1.01980 & -1.09781\end{array}$

h $\quad \begin{array}{llll}1.71435 & 0.48128 & 0.55104\end{array}$

h $\quad \begin{array}{llll}1.13033 & 2.14969 & 0.31369\end{array}$

h $\quad-2.31602 \quad-0.06590-0.51643$

$\begin{array}{llll}\text { h } & -2.79437 & -0.58452 & -2.50349\end{array}$

$\begin{array}{lllll}\text { h } & -2.09687 & 0.67798 & -3.58448\end{array}$

h $\quad-2.01451-1.54450-4.73008$

h $\quad-0.33583-1.00582-4.36016$

h $\quad-1.12473-2.26313-3.32866$

h $\quad-1.47643 \quad-6.254850 .02542$

h $\quad-1.68251 \quad-7.18453 \quad 2.37026$

h $\quad-1.87776-5.58463 \quad 4.30662$

h $\quad-1.88644 \quad-3.12486 \quad 3.91103$

\section{NH-EEEZ}

$E(B 3 L Y P /$ def2-TZVP $)=-1064.415662908$

35

$\begin{array}{lrrr}\mathrm{c} & -2.13512 & -5.63856 & 1.47655 \\ \mathrm{c} & -2.19442 & -4.96600 & 2.70939 \\ \mathrm{C} & -1.91531 & -3.59511 & 2.77718 \\ \mathrm{c} & -1.54806 & -2.87511 & 1.62699 \\ \mathrm{c} & -1.47019 & -3.56444 & 0.39222 \\ \mathrm{C} & -1.77961 & -4.93281 & 0.32262 \\ \mathrm{o} & -1.25959 & -1.54580 & 1.79962 \\ \mathrm{C} & -1.91465 & -0.60381 & 0.99222 \\ \mathrm{o} & -2.86300 & -0.87830 & 0.29184 \\ \mathrm{n} & -0.99957 & -2.91963 & -0.85583 \\ \mathrm{o} & -0.30548 & -1.90721 & -0.74963 \\ \mathrm{n} & -1.31388 & 0.61119 & 1.23367 \\ \mathrm{c} & -1.31667 & 1.84463 & 0.48375 \\ \mathrm{o} & -0.26702 & 2.48024 & 0.50655\end{array}$


C $\quad-2.51046 \quad 2.32307 \quad-0.22515$

$\begin{array}{llll}\text { C } & -3.76165 & 1.86497 & 0.07515\end{array}$

$\begin{array}{lllll}0 & -4.85929 & 2.36860 & -0.50988\end{array}$

c $\quad-6.11012 \quad 1.74422-0.17283$

C $\quad-7.21795 \quad 2.45941-0.92325$

C $\quad-2.27969 \quad 3.45142-1.19812$

$\begin{array}{llll}0 & -1.29549 & -3.46400 & -1.91675\end{array}$

$\begin{array}{llll}\text { h } & -0.43652 & 0.54101 & 1.76331\end{array}$

h $\quad-3.21979 \quad 3.72233-1.71868$

h $\quad-1.51521 \quad 3.17081-1.95523$

h $\quad-1.88610 \quad 4.35055-0.67405$

h $\quad-3.93460 \quad 1.06479 \quad 0.81279$

$\begin{array}{llll}\text { h } & -6.06367 & 0.66580 & -0.45100\end{array}$

$\begin{array}{llll}\text { h } & -6.26556 & 1.80877 & 0.93035\end{array}$

h $\quad-8.19921 \quad 1.99689-0.68288$

$\begin{array}{lllll}\text { h } & -7.25718 & 3.53359 & -0.64167\end{array}$

h $\quad-7.05777 \quad 2.39167-2.02064$

h $\quad-1.72135 \quad-5.41955-0.66175$

h $\quad-2.36311 \quad-6.71417 \quad 1.41376$

h $\quad-2.47213 \quad-5.50980 \quad 3.62669$

$\begin{array}{llll}\text { h } & -1.97905 & -3.04193 & 3.72675\end{array}$

\section{NH-EEZE}

$E(B 3 L Y P /$ def2-TZVP $)=-1064.414898082$

35

$\begin{array}{llll}\text { C } & -2.48502 & -5.28064 & 1.13663\end{array}$

$\begin{array}{llll}\text { C } & -1.89636 & -4.77068 & 2.30714\end{array}$

$\begin{array}{llll}\text { C } & -1.39868 & -3.46167 & 2.33218\end{array}$

$\begin{array}{llll}\text { C } & -1.45855 & -2.64713 & 1.18872\end{array}$

$\begin{array}{llll}\text { c } & -2.03100 & -3.17446 & 0.00567\end{array}$

c $\quad-2.55438-4.47823-0.00683$

$\begin{array}{lllll}0 & -0.90797 & -1.39567 & 1.30415\end{array}$

$\begin{array}{llll}\text { C } & -1.66892 & -0.30517 & 0.96111\end{array}$

$\begin{array}{lllll}0 & -2.85358 & -0.34096 & 0.70439\end{array}$

n $\quad-2.05143 \quad-2.43204-1.27475$

o $\quad-1.22832-1.52724-1.42685$

$\begin{array}{llll}\mathrm{n} & -0.91518 & 0.85697 & 0.95313\end{array}$

c $\quad 0.41951 \quad 1.10470 \quad 1.39585$

$\begin{array}{llll}\text { o } & 1.01249 & 0.34629 & 2.14731\end{array}$

C $\quad 1.011122 .372590 .89046$

$\begin{array}{llll}\text { c } & 0.59422 & 2.91204 & -0.29245\end{array}$

o $\quad 1.126824 .04116-0.79456$

c $\quad 0.77292 \quad 4.38061-2.14513$

C $\quad 1.32775 \quad 5.75878-2.45355$

C $\quad 2.16106 \quad 2.94034 \quad 1.67754$

o $\quad-2.86124-2.79613-2.12480$

$\begin{array}{llll}\text { h } & -1.46648 & 1.66382 & 0.65025\end{array}$

h $\quad 2.73203 \quad 3.67651 \quad 1.07703$

h $\quad 2.83319 \quad 2.11692 \quad 2.00069$

$\begin{array}{llll}\text { h } & 1.80690 & 3.44828 & 2.60419\end{array}$

h $\quad-0.18083 \quad 2.42746-0.91874$

h $\quad \begin{array}{llll}1.19472 & 3.61399 & -2.83721\end{array}$ 
h $\quad-0.33831 \quad 4.36119-2.25052$

$\begin{array}{llll}\text { h } & 1.07360 & 6.04517 & -3.49645\end{array}$

h $\quad 0.90140 \quad 6.51977-1.76526$

h $\quad 2.43364 \quad 5.77141-2.34640$

h $\quad-3.00596 \quad-4.83755-0.94264$

h $\quad-2.88760 \quad-6.30543 \quad 1.11263$

h $\quad-1.83228-5.39348 \quad 3.21390$

h $\quad-0.94988 \quad-3.02928 \quad 3.23924$

\section{NH-EEZZ}

$E(B 3 L Y P /$ def2-TZVP $)=-1064.414709522$

35

C $\quad-1.82717 \quad-2.44763 \quad 1.10980$

$\begin{array}{llll}\text { o } & -1.69556 & -1.08956 & 1.21055\end{array}$

$\begin{array}{llll}\text { C } & -2.10991 & -0.29274 & 0.11817\end{array}$

$\begin{array}{lllll}\mathrm{n} & -1.34379 & 0.85030 & 0.17591\end{array}$

$\begin{array}{lllll}0 & -2.95932 & -0.61707 & -0.66932\end{array}$

$\begin{array}{lllll}\text { C } & -1.47930 & 2.00356 & -0.65361\end{array}$

C $\quad-0.24205 \quad 2.81532-0.79002$

$\begin{array}{lllll}\text { o } & -2.53885 & 2.28817 & -1.18800\end{array}$

C $\quad-0.41508 \quad 4.24410 \quad-1.22948$

c $\quad 0.98636 \quad 2.23792-0.63199$

$\begin{array}{lllll}0 & 2.12868 & 2.93284 & -0.77960\end{array}$

C $\quad 3.34726 \quad 2.17053-0.80719$

c $\quad 4.51733 \quad 3.13532 \quad-0.76989$

$\begin{array}{llll}\text { C } & -0.66766 & -3.25401 & 1.01255\end{array}$

$\begin{array}{llll}\text { C } & -0.75967 & -4.65468 & 1.02467\end{array}$

$\begin{array}{llll}\text { C } & -2.01837 & -5.26387 & 1.07623\end{array}$

$\begin{array}{llll}\text { c } & -3.17746 & -4.46981 & 1.14308\end{array}$

$\begin{array}{llll}\text { C } & -3.08421 & -3.07095 & 1.16996\end{array}$

n $\quad 0.67827-2.653450 .89401$

$\begin{array}{llll}0 & 1.62520 & -3.30745 & 1.32761\end{array}$

$\begin{array}{llll}0 & 0.77524 & -1.55109 & 0.34598\end{array}$

$\begin{array}{llll}\text { h } & -0.54985 & 0.80232 & 0.81918\end{array}$

h $\quad 0.53144 \quad 4.65625-1.63349$

h $\quad-0.74174 \quad 4.89267-0.38393$

h $\quad-1.21060 \quad 4.30205-2.00306$

h $\quad \begin{array}{lllll}1.11087 & 1.15911 & -0.40992\end{array}$

$\begin{array}{llll}\text { h } & 3.36717 & 1.47610 & 0.06630\end{array}$

h $\quad 3.36808 \quad 1.55089-1.73456$

h $\quad 5.47473 \quad 2.57216-0.79803$

h $\quad 4.48996 \quad 3.82246-1.64280$

h $\quad 4.49616 \quad 3.74748 \quad 0.15720$

h $\quad 0.17015-5.24031 \quad 0.98243$

h $\quad-2.09602 \quad-6.36248 \quad 1.06552$

h $\quad-4.17031 \quad-4.94649 \quad 1.18302$

$\begin{array}{llll}\text { h } & -3.97998 & -2.43796 & 1.24373\end{array}$

2NH-EZEE

$E(B 3 L Y P /$ def2-TZVP) $=-1064.411293140$

http://mc.manuscriptcentral.com/poc 
35

$\begin{array}{rrrr}\text { c } & -2.25060 & -4.56877 & 2.15609 \\ \text { c } & -3.62476 & -4.31009 & 2.13171 \\ \text { c } & -4.09134 & -3.09752 & 1.59559 \\ \text { c } & -3.18395 & -2.14999 & 1.10504 \\ \text { c } & -1.80100 & -2.40160 & 1.11390 \\ \text { c } & -1.33980 & -3.63511 & 1.63414 \\ \text { o } & -1.00489 & -1.41785 & 0.58102 \\ \text { c } & 0.07589 & -0.95563 & 1.31452 \\ \text { h } & 0.82130 & -0.05054 & 0.58426 \\ \text { c } & 0.56859 & 0.65994 & -0.63989 \\ \text { c } & 0.06780 & -0.07848 & -1.81875 \\ \text { c } & 0.19256 & -1.56652 & -2.03497 \\ \text { h } & 0.08315 & -4.04195 & 1.60327 \\ \text { o } & 0.44657 & -4.88097 & 2.42239 \\ \text { o } & 0.29276 & -1.25981 & 2.46694 \\ \text { o } & 0.79945 & -3.55230 & 0.72817 \\ \text { o } & 0.85695 & 1.85482 & -0.64280 \\ \text { c } & -0.43806 & 0.72769 & -2.80046 \\ \text { o } & -0.88360 & 0.22551 & -3.96125 \\ \text { c } & -1.37925 & 1.16722 & -4.92817 \\ \text { c } & -1.85747 & 0.39605 & -6.14424 \\ \text { h } & 1.44750 & 0.48802 & 1.19403 \\ \text { h } & -0.79692 & -2.07449 & -2.02756 \\ \text { h } & 0.81945 & -2.05184 & -1.26270 \\ \text { h } & 0.64655 & -1.76071 & -3.03244 \\ \text { h } & -0.48642 & 1.82465 & -2.66433 \\ \text { h } & -2.20987 & 1.75612 & -4.47236 \\ \text { h } & -0.56288 & 1.87761 & -5.19720 \\ \text { h } & -2.24411 & 1.09969 & -6.91219 \\ \text { h } & -1.02586 & -0.18926 & -6.59181 \\ \text { h } & -2.67173 & -0.30851 & -5.87047 \\ \text { h } & -1.84276 & -5.50194 & 2.57075 \\ \text { h } & -4.33090 & -5.05501 & 2.53076 \\ \text { h } & -5.17116 & -2.87918 & 1.57097 \\ \text { h } & -3.52299 & -1.18127 & 0.70739\end{array}$

\section{NH-EZEZ}

$E(B 3 L Y P / d e f 2-T Z V P)=-1064.415459278$

35

$\begin{array}{lrrr}\text { c } & -1.13269 & -4.52105 & 0.03088 \\ \mathrm{c} & -1.82126 & -5.43110 & 0.83906 \\ \mathrm{c} & -2.59893 & -4.95657 & 1.90960 \\ \mathrm{c} & -2.69533 & -3.58086 & 2.15393 \\ \mathrm{c} & -2.00218 & -2.65483 & 1.35516 \\ \mathrm{c} & -1.20507 & -3.14233 & 0.29038 \\ \mathrm{o} & -2.12985 & -1.33234 & 1.69361 \\ \mathrm{c} & -2.54519 & -0.42750 & 0.70475 \\ \mathrm{n} & -2.34807 & 0.83979 & 1.20708 \\ \mathrm{c} & -2.49367 & 2.09559 & 0.53327 \\ \mathrm{c} & -3.65203 & 2.28448 & -0.36589\end{array}$




\section{NH-EZZE}

$E(B 3 L Y P /$ def2-TZVP $)=-1064.416583725$

35

$\begin{array}{llll}\text { C } & -2.58855 & -5.16397 & 0.56608\end{array}$

$\begin{array}{llll}\text { C } & -2.80557 & -5.38741 & 1.92947\end{array}$

$\begin{array}{llll}\text { C } & -2.38371 & -4.42615 & 2.86453\end{array}$

C $\quad-1.76695 \quad-3.24564 \quad 2.43154$

$\begin{array}{llll}\text { c } & -1.53894 & -3.01080 & 1.06477\end{array}$

$\begin{array}{llll}\text { c } & -1.94401 & -3.99441 & 0.12983\end{array}$

$\begin{array}{lllll}0 & -0.89143 & -1.84314 & 0.74991\end{array}$

$\begin{array}{llll}\text { c } & -1.45296 & -1.00569 & -0.18060\end{array}$

$\begin{array}{lllll}\mathrm{n} & -0.60908 & 0.04296 & -0.51129\end{array}$

c $\quad 0.692850 .38210-0.05485$

C $\quad 1.26806 \quad 1.56149-0.76422$

$\begin{array}{lllll}\text { C } & 0.55641 & 2.29793 & -1.87209\end{array}$

n $\quad-1.66016-3.88872-1.31933$

$\begin{array}{llll}0 & -2.34435 & -4.57131 & -2.07882\end{array}$

o $\quad-2.56838-1.13305-0.64027$

o $\quad-0.72957-3.16104-1.66865$

$\begin{array}{lllll}0 & 1.26225 & -0.23627 & 0.83464\end{array}$

$\begin{array}{llll}\text { C } & 2.51081 & 1.92712 & -0.33612\end{array}$

$\begin{array}{llll}\text { o } & 3.18511 & 2.96356 & -0.85606\end{array}$

C $\quad 4.49920 \quad 3.20826-0.32465$

c $\quad 5.06645 \quad 4.44295-0.99949$

h $\quad-1.033240 .64383-1.22256$

h $\quad \begin{array}{lllll}1.19492 & 3.11911 & -2.25740\end{array}$

h $\quad-0.39877 \quad 2.76195 \quad-1.52932$ 


$\begin{array}{lrrr}\text { h } & 0.31864 & 1.63268 & -2.73461 \\ \text { h } & 2.99651 & 1.35229 & 0.47529 \\ \text { h } & 4.42625 & 3.35032 & 0.77924 \\ \text { h } & 5.14062 & 2.31584 & -0.51412 \\ \text { h } & 6.08535 & 4.65403 & -0.60961 \\ \text { h } & 5.13564 & 4.29586 & -2.09864 \\ \text { h } & 4.42566 & 5.32950 & -0.80500 \\ \text { h } & -2.90193 & -5.88813 & -0.19953 \\ \text { h } & -3.30071 & -6.31317 & 2.26227 \\ \text { h } & -2.54529 & -4.59068 & 3.94217 \\ \text { h } & -1.44501 & -2.46833 & 3.14097\end{array}$

2NH-EZZZ

$E(B 3 L Y P /$ def2-TZVP $)=-1064.416538143$

35

C $\quad-3.15636 \quad-3.13016 \quad 1.39830$

$\begin{array}{llll}\text { c } & -1.83191 & -2.79497 & 1.06640\end{array}$

c $\quad-0.91002 \quad-3.83483 \quad 0.80177$

C $\quad-1.29204 \quad-5.18122 \quad 0.90463$

$\begin{array}{llll}\text { C } & -2.61629 & -5.50371 & 1.22133\end{array}$

C $\quad-3.54484-4.47587 \quad 1.46310$

o $\quad-1.38705-1.50006 \quad 1.06615$

$\begin{array}{llll}\text { c } & -2.00253 & -0.55395 & 0.22328\end{array}$

$\begin{array}{lllll}0 & -3.07857 & -0.69894 & -0.29446\end{array}$

n $\quad 0.48551 \quad-3.54448 \quad 0.40120$

o $\quad 0.70252-2.51201-0.23376$

$\begin{array}{lllll}\mathrm{n} & -1.12214 & 0.50792 & 0.15098\end{array}$

C $\quad-1.36131 \quad 1.75526-0.48612$

$\begin{array}{lllll}\text { o } & -2.45790 & 2.04714 & -0.94185\end{array}$

$\begin{array}{llll}\text { c } & -0.15799 & 2.63227 & -0.52462\end{array}$

c $\quad-0.359893 .84203 \quad-1.12250$

$\begin{array}{lllll}0 & 0.60733 & 4.76196 & -1.24943\end{array}$

C $\quad 0.25983 \quad 5.97218-1.94480$

C $\quad 1.46887 \quad 6.88843-1.94384$

$\begin{array}{llll}\text { C } & 1.18238 & 2.22654 & 0.03617\end{array}$

$\begin{array}{llll}\text { o } & 1.33698 & -4.37878 & 0.70957\end{array}$

$\begin{array}{lllll}\text { h } & -0.19567 & 0.32441 & 0.54583\end{array}$

h $\quad \begin{array}{llll}1.93026 & 3.02559 & -0.14373\end{array}$

h $\quad 1.57285 \quad 1.29555-0.43664$

h $\quad \begin{array}{llll}1.14642 & 2.05510 & 1.13803\end{array}$

h $\quad-1.36147 \quad 4.08799-1.52494$

h $\quad-0.05104 \quad 5.71789-2.98529$

h $\quad-0.60887 \quad 6.45003-1.43428$

h $\quad \begin{array}{llll}1.22990 & 7.83214 & -2.47949\end{array}$

h $\quad 1.77256 \quad 7.14185-0.90549$

h $\quad 2.33092 \quad 6.40575 \quad-2.45221$

$\begin{array}{llll}\text { h } & -0.53100 & -5.95366 & 0.72166\end{array}$

h $\quad-2.92397 \quad-6.55944 \quad 1.28297$

h $\quad-4.58842-4.72355 \quad 1.71639$

$\begin{array}{llll}\text { h } & -3.87081 & -2.32193 & 1.60657\end{array}$

2NH-ZEEE

http://mc.manuscriptcentral.com/poc 
35

C $\quad-2.03893 \quad-5.90231 \quad 1.58221$

$\begin{array}{llll}\text { C } & -2.45482 & -5.22441 & 2.74014\end{array}$

C $\quad-2.25015 \quad-3.84388 \quad 2.88550$

C $\quad-1.59976-3.11709 \quad 1.86978$

C $\quad-1.15960-3.81795 \quad 0.72222$

$\begin{array}{llll}\text { C } & -1.39591 & -5.19040 & 0.56311\end{array}$

$\begin{array}{lllll}\text { o } & -1.34959 & -1.76787 & 1.92114\end{array}$

C $\quad-2.26029-0.890792 .48729$

o $\quad-3.26825-1.218093 .07830$

n $\quad-0.42419-3.12040-0.34621$

$\begin{array}{llll}0 & 0.53323 & -2.42676 & -0.01807\end{array}$

$\begin{array}{lllll}\mathrm{n} & -1.82741 & 0.41375 & 2.36186\end{array}$

c $\quad-\quad-0.78804 \quad 1.04709 \quad 1.61359$

$\begin{array}{llll}0 & -0.25787 & 2.03413 & 2.11403\end{array}$

$\begin{array}{llll}\text { C } & -0.45086 & 0.57540 & 0.25220\end{array}$

c $\quad-1.33168 \quad-0.03034-0.59864$

$\begin{array}{lllll}0 & -2.58047 & -0.41095 & -0.27296\end{array}$

$\begin{array}{llll}\text { C } & -3.37766 & -1.00277 & -1.31840\end{array}$

$\begin{array}{llll}\text { C } & -4.75627 & -1.29044 & -0.75398\end{array}$

C $\quad 0.91443 \quad 0.98084-0.24589$

o $\quad-0.81795-3.29925-1.50523$

h $\quad-2.25053 \quad 1.04050 \quad 3.05516$

h $\quad 1.07020 \quad 0.66600 \quad-1.29901$

$\begin{array}{llll}\text { h } & 1.71293 & 0.51030 & 0.37003\end{array}$

h $\quad \begin{array}{llll}1.05165 & 2.08155 & -0.17100\end{array}$

h $\quad-1.02251-0.24844-1.63988$

h $\quad-3.43308-0.29360-2.17737$

h $\quad-2.87527 \quad-1.93500-1.66749$

h $\quad-5.38806-1.77397-1.52975$

h $\quad-4.69130 \quad-1.97113 \quad 0.12218$

h $\quad-5.25536-0.35352-0.42610$

h $\quad-1.05946 \quad-5.67986-0.36323$

h $\quad-2.21165 \quad-6.98453 \quad 1.47310$

h $\quad-2.95843 \quad-5.77590 \quad 3.55050$

h $\quad-2.60564 \quad-3.31635 \quad 3.77862$

\section{NH-ZEEZ}

$E(B 3 L Y P /$ def2-TZVP $)=-1064.411404609$

35

$\begin{array}{llll}\text { C } & -2.48514 & -4.94612 & 2.51132\end{array}$

C $\quad-2.20667 \quad-3.577692 .62077$

$\begin{array}{llll}\text { C } & -1.59824 & -2.87708 & 1.56431\end{array}$

$\begin{array}{llll}\text { C } & -1.27656 & -3.58448 & 0.38041\end{array}$

$\begin{array}{llll}\text { C } & -1.58382 & -4.94989 & 0.26243\end{array}$

$\begin{array}{llll}\text { c } & -2.18080 & -5.63592 & 1.32498\end{array}$

$\begin{array}{llll}\text { o } & -1.33604 & -1.54912 & 1.77801\end{array}$

C $\quad-1.81312-0.61254 \quad 0.84660$

$\begin{array}{llll}\mathrm{n} & -1.23321 & 0.59368 & 1.16730\end{array}$ 


$$
\begin{array}{rrr}
-1.33085 & 1.86002 & 0.52393 \\
-2.55041 & 2.23480 & -0.22825 \\
-2.33267 & 3.26001 & -1.31542 \\
-0.55106 & -2.96012 & -0.74993 \\
-0.63139 & -3.51246 & -1.84450 \\
-2.61072 & -0.88280 & -0.01920 \\
0.12030 & -1.95477 & -0.51222 \\
-0.37017 & 2.61578 & 0.63292 \\
-3.81391 & 1.84768 & 0.10718 \\
-4.12630 & 1.09213 & 1.17314 \\
-5.38833 & 0.40926 & 1.12060 \\
-5.64045 & -0.23790 & 2.46967 \\
-0.40933 & 0.53763 & 1.77697 \\
-3.28575 & 3.51549 & -1.82549 \\
-1.61589 & 2.88796 & -2.08105 \\
-1.89399 & 4.19182 & -0.89555 \\
-4.67749 & 2.20665 & -0.49127 \\
-6.19072 & 1.14461 & 0.86856 \\
-5.34650 & -0.35585 & 0.31222 \\
-6.62146 & -0.75987 & 2.46298 \\
-4.85045 & -0.98528 & 2.69924 \\
-5.65144 & 0.52286 & 3.27996 \\
-1.33000 & -5.45024 & -0.68342 \\
-2.40577 & -6.70965 & 1.22787 \\
-2.95416 & -5.47475 & 3.35690 \\
-2.45568 & -3.01107 & 3.53140
\end{array}
$$

\section{NH-ZEZE}

$E(B 3 L Y P /$ def2-TZVP) $=-1064.416351357$

35

$\begin{array}{lrrr}\text { c } & -2.50797 & -4.46180 & 2.71627 \\ \text { c } & -1.96419 & -3.18148 & 2.55572 \\ \text { c } & -1.60569 & -2.70310 & 1.28316 \\ \text { c } & -1.80469 & -3.54560 & 0.16186 \\ \text { c } & -2.37727 & -4.81835 & 0.32386 \\ \text { c } & -2.72484 & -5.28320 & 1.59606 \\ \text { o } & -1.04311 & -1.45439 & 1.24159 \\ \text { c } & -1.55874 & -0.51996 & 0.37384 \\ \text { n } & -0.77317 & 0.61545 & 0.30310 \\ \text { c } & 0.45494 & 0.93568 & 0.92456 \\ \text { c } & 1.08486 & 2.21883 & 0.47389 \\ \text { c } & 2.38698 & 2.53776 & 1.16448 \\ \text { n } & -1.36975 & -3.17877 & -1.20415 \\ \text { o } & -1.89805 & -3.77194 & -2.14266 \\ \text { o } & -2.60160 & -0.65094 & -0.23367 \\ \text { o } & -0.47650 & -2.33755 & -1.31478 \\ \text { o } & 0.98804 & 0.23346 & 1.77198 \\ \text { c } & 0.60081 & 3.07084 & -0.47445 \\ \text { o } & -0.58317 & 2.91884 & -1.11795 \\ \text { c } & -0.79741 & 3.69727 & -2.30946 \\ \text { c } & -2.24319 & 3.53293 & -2.73811 \\ \text { h } & -1.13894 & 1.30568 & -0.36729\end{array}$


2NH-ZEZZ

$E(B 3 L Y P /$ def2-TZVP $)=-1064.414956284$

35

$\begin{array}{llll}\text { C } & -3.21875 & -3.07221 & 0.94924\end{array}$

$\begin{array}{llll}\text { C } & -1.85405 & -2.74149 & 0.81641\end{array}$

$\begin{array}{llll}\text { C } & -0.88974 & -3.76149 & 1.03728\end{array}$

$\begin{array}{llll}\text { C } & -1.27150 & -5.05898 & 1.40801\end{array}$

c $\quad-2.62856 \quad-5.37972 \quad 1.51684$

$\begin{array}{llll}\text { C } & -3.59100 & -4.38275 & 1.28318\end{array}$

$\begin{array}{lllll}0 & -1.36417 & -1.49577 & 0.56143\end{array}$

C $\quad-2.10655-0.41500 \quad 0.05389$

o $\quad-3.30036-0.39838-0.11160$

n $\quad 0.55543 \quad-3.49574 \quad 0.88863$

o $\quad 0.90701-2.71796-0.00126$

$\begin{array}{lllll}\mathrm{n} & -1.17112 & 0.57054 & -0.18653\end{array}$

$\begin{array}{lllll}\text { c } & -1.44407 & 1.89911 & -0.59017\end{array}$

$\begin{array}{lllll}0 & -2.57900 & 2.31708 & -0.76509\end{array}$

C $\quad-0.24586 \quad 2.77380-0.76819$

$\begin{array}{llll}\text { C } & 1.05774 & 2.40735 & -0.59457\end{array}$

$\begin{array}{lllll}0 & 1.48096 & 1.15760 & -0.29728\end{array}$

$\begin{array}{llll}\text { C } & 2.84486 & 0.97425 & 0.13913\end{array}$

c $\quad 3.36885-0.35566-0.36981$

c $\quad-0.57394 \quad 4.18993-1.17171$

$\begin{array}{llll}\text { o } & 1.31784 & -4.09316 & 1.64817\end{array}$

h $\quad-0.182020 .30834-0.06198$

h $\quad 0.34288 \quad 4.80971-1.26810$

h $\quad-1.25085 \quad 4.66680-0.42919$

h $\quad-1.118124 .21006-2.14178$

h $\quad \begin{array}{llll}1.86755 & 3.15340 & -0.72549\end{array}$

$\begin{array}{llll}\text { h } & 3.45437 & 1.82649 & -0.24215\end{array}$

$\begin{array}{llll}\text { h } & 2.85522 & 1.01807 & 1.25287\end{array}$

h $\quad 4.40173 \quad-0.51428 \quad 0.01030$

h $\quad 2.73580-1.20399-0.03021$

h $\quad 3.39997-0.36860-1.48051$

h $\quad-0.48070 \quad-5.79959 \quad 1.59764$

$\begin{array}{llll}\text { h } & -2.93374 & -6.40259 & 1.78740\end{array}$

h $\quad-4.66335 \quad-4.62165 \quad 1.37232$

$\begin{array}{llll}\text { h } & -3.97073 & -2.29090 & 0.78668\end{array}$ 
2NH-ZZEE

$E(B 3 L Y P / d e f 2-T Z V P)=-1064.405006331$

35

C $\quad-3.83472 \quad-3.96928 \quad 1.60046$

C $\quad-4.14030-2.71986 \quad 1.03383$

$\begin{array}{llll}\text { C } & -3.11129 & -1.84493 & 0.66248\end{array}$

$\begin{array}{llll}\text { C } & -1.76355 & -2.20778 & 0.82438\end{array}$

$\begin{array}{llll}\text { C } & -1.46284 & -3.47610 & 1.37547\end{array}$

C $\quad-2.49712-4.33783 \quad 1.77671$

$\begin{array}{lllll}0 & -0.83200 & -1.29234 & 0.39751\end{array}$

C $\quad 0.15098 \quad-0.88158 \quad 1.27979$

o $\quad 0.19069-1.198242 .44804$

$\begin{array}{lllll}\mathrm{n} & -0.08066 & -3.98617 & 1.50901\end{array}$

$\begin{array}{llll}\text { o } & 0.77335 & -3.53966 & 0.73919\end{array}$

n $\quad 1.03890-0.01292 \quad 0.67169$

$\begin{array}{llll}\text { c } & 1.06600 & 0.64092 & -0.59975\end{array}$

$\begin{array}{lllll}0 & & 1.62925 & 1.72347 & -0.65711\end{array}$

$\begin{array}{llll}\text { C } & 0.50947 & -0.07992 & -1.78436\end{array}$

$\begin{array}{llll}\text { c } & -0.32839 & 0.57824 & -2.62837\end{array}$

$\begin{array}{lllll}0 & -0.85368 & 1.79148 & -2.36201\end{array}$

C $\quad-1.39601 \quad 2.51294 \quad-3.47149$

$\begin{array}{lllll}\text { C } & -2.13506 & 3.72777 & -2.93948\end{array}$

c $\quad 1.00078-1.47468-2.09224$

$\begin{array}{lllll}0 & 0.11915 & -4.85730 & 2.35017\end{array}$

$\begin{array}{llll}\text { h } & 1.64740 & 0.45400 & 1.35461\end{array}$

h $\quad 0.56438-1.83812-3.04782$

h $\quad 0.74152-2.20613-1.29649$

h $\quad 2.10979-1.49013-2.20202$

h $\quad-0.67193 \quad 0.09766-3.56880$

h $\quad-0.56717 \quad 2.82003 \quad-4.15301$

h $\quad-2.08143 \quad 1.84610-4.05107$

h $\quad-2.55478 \quad 4.31947 \quad-3.78130$

h $\quad-2.96910 \quad 3.42235-2.27153$

h $\quad-1.44650 \quad 4.37904-2.35989$

h $\quad-2.21408 \quad-5.30273 \quad 2.22167$

h $\quad-4.63814 \quad-4.65760 \quad 1.90625$

h $\quad-5.18979-2.415620 .89153$

$\begin{array}{llll}\text { h } & -3.32403 & -0.85007 & 0.24233\end{array}$

2NH-ZZEZ

$E(B 3 L Y P /$ def2-TZVP $)=-1064.407877132$

35

$\begin{array}{llll}\text { C } & -1.82292 & -5.37644 & 0.61384\end{array}$

C $\quad-2.52263 \quad-4.97190 \quad 1.76396$

$\begin{array}{llll}\text { c } & -2.61366 & -3.61294 & 2.09076\end{array}$

C $\quad-1.98986-2.635621 .29582$

$\begin{array}{llll}\text { c } & -1.26960 & -3.05314 & 0.14996\end{array}$

C $\quad-1.20528-4.41412-0.19152$

http://mc.manuscriptcentral.com/poc 
2NH-ZZZE

$E(B 3 L Y P /$ def2-TZVP $)=-1064.407500936$

35

C $\quad-2.87298 \quad-5.40656 \quad 1.88978$

C $\quad-2.44480-4.46643 \quad 2.84336$

$\begin{array}{llll}\text { c } & -1.80862 & -3.28782 & 2.43415\end{array}$

C $\quad-1.56707 \quad-3.03359 \quad 1.07292$

c $\quad-1.97885-3.99666 \quad 0.11939$

$\begin{array}{llll}\text { C } & -2.64267 & -5.16410 & 0.53189\end{array}$

$\begin{array}{lllll}0 & -0.90130 & -1.87079 & 0.78233\end{array}$

C $\quad-1.44376-1.01022-0.13979$

$\begin{array}{llll}0 & -2.55903 & -1.11636 & -0.60687\end{array}$

n $\quad-1.68236-3.87228-1.32558$

o $\quad-0.73954-3.15170-1.65659$

$\begin{array}{lllll}\mathrm{n} & -0.58301 & 0.02838 & -0.45229\end{array}$

$\begin{array}{llll}\text { c } & 0.72673 & 0.34278 & 0.02595\end{array}$

$\begin{array}{lllll}0 & 1.26073 & -0.28665 & 0.92382\end{array}$

$\begin{array}{lllll}\text { C } & 1.33367 & 1.50568 & -0.68351\end{array}$

$\begin{array}{llll}\text { C } & 2.57296 & 1.96285 & -0.32014\end{array}$

$\begin{array}{llll}\text { o } & 3.33480 & 1.45778 & 0.65246\end{array}$

C $\quad 4.59766 \quad 2.10144 \quad 0.88458$

C $\quad 5.32105 \quad 1.34037 \quad 1.97968$ 
C $\quad 0.61963 \quad 2.20246-1.82241$

o $\quad-2.36907-4.53409-2.10118$

h $\quad-0.99773 \quad 0.64101-1.15840$

h $\quad 1.24084 \quad 3.02451-2.23842$

h $\quad-0.34396 \quad 2.66745-1.50358$

h $\quad 0.39604 \quad 1.51093 \quad-2.66798$

h $\quad 3.00673 \quad 2.82644 \quad-0.86761$

h $\quad 5.18953 \quad 2.10416-0.06160$

h $\quad 4.42006 \quad 3.16246 \quad 1.18252$

h $\quad \begin{array}{llll}6.30353 & 1.81559 & 2.18905\end{array}$

$\begin{array}{llll}\text { h } & 4.72199 & 1.33648 & 2.91497\end{array}$

h $\quad \begin{array}{llll}5.49391 & 0.28614 & 1.67548\end{array}$

h $\quad-2.96009-5.87143 \quad-0.24762$

h $\quad-3.38341-6.33056 \quad 2.20387$

h $\quad-2.61693 \quad-4.64600 \quad 3.91699$

h $\quad-1.48131 \quad-2.52644 \quad 3.15825$

2NH-ZZZZ

$E(B 3 L Y P / d e f 2-T Z V P)=-1064.407425375$

35

C $\quad-3.59200 \quad-4.21762 \quad 1.65225$

C $\quad-3.14556-2.89149 \quad 1.56180$

$\begin{array}{llll}\text { c } & -1.84623 & -2.61661 & 1.09817\end{array}$

$\begin{array}{llll}\text { C } & -1.01041 & -3.69812 & 0.73051\end{array}$

$\begin{array}{llll}\text { C } & -1.44719 & -5.02541 & 0.85954\end{array}$

C $\quad-2.74683 \quad-5.286991 .30690$

$\begin{array}{llll}0 & -1.33007 & -1.35064 & 1.06581\end{array}$

$\begin{array}{llll}\text { c } & -2.00576 & -0.32379 & 0.37341\end{array}$

$\begin{array}{lllll}\text { n } & -1.07169 & 0.67639 & 0.19854\end{array}$

c $\quad-1.32393 \quad 1.97932-0.33210$

c $\quad-0.07879 \quad 2.76931-0.53984$

$\begin{array}{llll}\text { C } & 1.28600 & 2.18441 & -0.24378\end{array}$

n $\quad 0.34976-3.47239 \quad 0.19111$

$\begin{array}{llll}0 & 1.18597 & -4.35012 & 0.40757\end{array}$

$\begin{array}{lllll}0 & -3.15981 & -0.37181 & 0.03639\end{array}$

$\begin{array}{lllll}0 & 0.55260 & -2.44680 & -0.45958\end{array}$

$\begin{array}{lllll}0 & -2.45670 & 2.36060 & -0.57074\end{array}$

C $\quad-0.14913 \quad 4.05085-1.01924$

$\begin{array}{lllll}0 & -1.27171 & 4.70374 & -1.32577\end{array}$

$\begin{array}{llll}\text { C } & -1.12667 & 6.03916 & -1.83561\end{array}$

$\begin{array}{llll}\text { C } & -2.51218 & 6.58734 & -2.12140\end{array}$

$\begin{array}{lllll}\text { h } & -0.11251 & 0.40466 & 0.42962\end{array}$

h $\quad 2.09146 \quad 2.90872-0.49071$

$\begin{array}{llll}\text { h } & 1.49124 & 1.26251 & -0.83694\end{array}$

$\begin{array}{llll}\text { h } & 1.41517 & 1.92466 & 0.83408\end{array}$

h $\quad 0.79329 \quad 4.61754-1.17526$

h $\quad-0.59334 \quad 6.66515-1.08085$

h $\quad-0.50562 \quad 6.01344-2.76251$

h $\quad-2.43788 \quad 7.62233-2.51896$

h $\quad-3.03611 \quad 5.95571-2.86988$

h $\quad-3.12490 \quad 6.60509-1.19522$

$\begin{array}{llll}\text { h } & -0.74827 & -5.83144 & 0.59297\end{array}$ 
h $\quad-3.09986 \quad-6.32692 \quad 1.39027$

h $\quad-4.61543 \quad-4.416942 .00949$

$\begin{array}{llll}\text { h } & -3.79379 & -2.05321 & 1.85037\end{array}$

\section{OH-EEEE}

$E(B 3 L Y P /$ def2-TZVP $)=-1064.385454852$

35

C $\quad-1.94905 \quad-5.28329 \quad 1.24726$

C $\quad-2.11043 \quad-5.82504 \quad 2.53519$

$\begin{array}{llll}\text { C } & -1.74198 & -5.09025 & 3.66497\end{array}$

C $\quad-1.20940-3.79798 \quad 3.50696$

C $\quad-1.06724-3.24508 \quad 2.22906$

$\begin{array}{llll}\text { c } & -1.43837 & -3.96918 & 1.08221\end{array}$

o $\quad-1.20973-3.34602-0.11898$

C $\quad-2.17976-3.21276-1.07179$

o $\quad-3.41830-3.35739-0.55508$

$\begin{array}{llll}\mathrm{n} & -2.27480 & -6.16397 & 0.10840\end{array}$

o $\quad-1.87097-5.82468-1.01005$

n $\quad-1.95491-2.93083-2.28564$

c $\quad-0.64990-2.69181-2.80799$

$\begin{array}{lllll}0 & -0.01921 & -1.68418 & -2.50181\end{array}$

C $\quad-0.14393-3.68733-3.77425$

C $\quad-0.90157-4.78371-4.06915$

o $\quad-0.49494-5.74939-4.91257$

C $\quad-1.36306-6.88536-5.05721$

C $\quad-1.22406-7.87714-3.90880$

$\begin{array}{llll}\text { C } & 1.22017 & -3.44062 & -4.35773\end{array}$

$\begin{array}{llll}\text { o } & -2.89688 & -7.19823 & 0.33870\end{array}$

h $\quad \begin{array}{llll}1.50424 & -4.24590 & -5.06517\end{array}$

h $\quad 1.99010-3.37721-3.55637$

h $\quad 1.24943-2.46393-4.89003$

h $\quad-1.90426 \quad-4.91083-3.62094$

h $\quad-2.41672 \quad-6.53232-5.15804$

h $\quad-1.06549-7.34602-6.02274$

h $\quad-1.85369-8.77309-4.10580$

h $\quad-0.16826-8.20989-3.80800$

h $\quad-1.54790-7.43206-2.94235$

h $\quad-2.52153 \quad-6.84176 \quad 2.61065$

h $\quad-1.86391 \quad-5.52744 \quad 4.66829$

h $\quad-0.90977 \quad-3.20904 \quad 4.38882$

h $\quad-0.66820-2.23036 \quad 2.07931$

h $\quad-4.03972 \quad-3.23035-1.30944$

\section{OH-EEZE}

$E(B 3 L Y P /$ def2-TZVP $)=-1064.403330874$

35

$\begin{array}{llll}\text { C } & -1.76843 & -4.60902 & 1.68783 \\ \text { C } & -2.38398 & -3.84963 & 2.68604\end{array}$ 


$\begin{array}{lrrr}\text { C } & -3.03136 & -2.64792 & 2.34301 \\ \text { C } & -3.07176 & -2.22224 & 1.01024 \\ \text { C } & -2.44790 & -2.96558 & -0.01017 \\ \text { C } & -1.78168 & -4.16522 & 0.35312 \\ \text { o } & -2.50023 & -2.48003 & -1.27711 \\ \text { C } & -3.13916 & -3.29999 & -2.31118 \\ \text { n } & -2.96643 & -2.74115 & -3.52700 \\ \text { C } & -1.86107 & -2.16819 & -3.94626 \\ \text { C } & -1.82511 & -1.37886 & -5.17930 \\ \text { c } & -0.53665 & -0.73717 & -5.62062 \\ \text { h } & -1.04079 & -4.98032 & -0.62153 \\ \text { o } & -0.82505 & -6.15287 & -0.34186 \\ \text { o } & -3.78472 & -4.27000 & -1.98870 \\ \text { o } & -0.62660 & -4.44159 & -1.66370 \\ \text { o } & -0.66648 & -2.26643 & -3.35223 \\ \text { C } & -2.99027 & -1.27180 & -5.88679 \\ \text { o } & -3.07995 & -0.55879 & -7.01761 \\ \text { C } & -4.34197 & -0.59688 & -7.70745 \\ \text { C } & -4.26427 & 0.34035 & -8.89803 \\ \text { h } & -0.67995 & -0.19325 & -6.57589 \\ \text { h } & 0.26291 & -1.49794 & -5.76019 \\ \text { h } & -0.15951 & -0.02015 & -4.85778 \\ \text { h } & -3.90241 & -1.78740 & -5.53084 \\ \text { h } & -4.54723 & -1.64394 & -8.03170 \\ \text { h } & -5.15171 & -0.29049 & -7.00448 \\ \text { h } & -5.22714 & 0.32913 & -9.45227 \\ \text { h } & -4.06029 & 1.38187 & -8.56877 \\ \text { h } & -3.45578 & 0.02912 & -9.59364 \\ \text { h } & -1.25432 & -5.55439 & 1.91313 \\ \text { h } & -2.35877 & -4.19395 & 3.73178 \\ \text { h } & -3.52221 & -2.04224 & 3.12205 \\ \text { h } & -3.59583 & -1.30040 & 0.71531 \\ \text { h } & -0.72800 & -2.84807 & -2.53859\end{array}$

\section{OH-EZZE}

$E(B 3 L Y P /$ def2-TZVP $)=-1064.404100222$

35

$\begin{array}{llll}\mathrm{c} & -2.85624 & -2.70420 & 2.46798 \\ \mathrm{c} & -2.89226 & -2.20614 & 1.16042 \\ \mathrm{c} & -2.40996 & -2.96726 & 0.07850 \\ \mathrm{c} & -1.89060 & -4.25932 & 0.35236 \\ \mathrm{c} & -1.88550 & -4.77235 & 1.66215 \\ \mathrm{c} & -2.35840 & -3.99554 & 2.72271 \\ \mathrm{o} & -2.45047 & -2.40831 & -1.15902 \\ \mathrm{n} & -1.29413 & -5.10876 & -0.69008 \\ \mathrm{o} & -1.23242 & -6.31348 & -0.48086 \\ \mathrm{o} & -0.83586 & -4.57172 & -1.71435 \\ \mathrm{c} & -3.18912 & -3.09786 & -2.21196 \\ \mathrm{n} & -3.01116 & -2.46497 & -3.39440 \\ \mathrm{c} & -1.85904 & -2.03905 & -3.85030 \\ \mathrm{c} & -1.83710 & -1.18683 & -5.03832 \\ \mathrm{c} & -3.14628 & -0.76898 & -5.65091\end{array}$


35

$\begin{array}{rrrr}\text { c } & -3.22273 & -2.89605 & 0.80182 \\ \mathrm{c} & -1.83770 & -2.68710 & 0.71951 \\ \mathrm{c} & -0.94818 & -3.73918 & 1.02211 \\ \mathrm{c} & -1.49470 & -4.99012 & 1.41485 \\ \mathrm{c} & -2.87831 & -5.17841 & 1.52794 \\ \mathrm{c} & -3.75174 & -4.13327 & 1.20505 \\ \mathrm{o} & 0.41336 & -3.66363 & 1.04048 \\ \mathrm{c} & 1.19640 & -2.64118 & 0.55659 \\ \mathrm{n} & 2.49676 & -2.97468 & 0.70197 \\ \mathrm{c} & 3.41759 & -2.10865 & 0.29288 \\ \mathrm{c} & 4.83963 & -2.41041 & 0.38532 \\ \mathrm{c} & 5.82027 & -1.33506 & -0.01946 \\ \mathrm{n} & -0.62474 & -6.14918 & 1.72156 \\ \mathrm{o} & -0.97597 & -6.86856 & 2.65857 \\ \mathrm{o} & 0.70942 & -1.59686 & 0.07173 \\ \mathrm{o} & 0.35815 & -6.33437 & 1.00787 \\ \mathrm{o} & 3.13246 & -0.91122 & -0.22490 \\ \mathrm{c} & 5.30707 & -3.63806 & 0.79913 \\ \mathrm{o} & 4.54920 & -4.67848 & 1.11420 \\ \mathrm{c} & 5.15407 & -5.86973 & 1.64199 \\ \mathrm{c} & 4.29709 & -7.05574 & 1.23395 \\ \mathrm{~h} & 6.86870 & -1.67732 & 0.11330 \\ \mathrm{~h} & 5.68004 & -0.40892 & 0.58113 \\ \mathrm{~h} & 5.68512 & -1.04174 & -1.08452 \\ \mathrm{~h} & 6.40513 & -3.80107 & 0.85628 \\ \mathrm{~h} & 6.20006 & -5.96047 & 1.26185 \\ \mathrm{~h} & 5.19770 & -5.76807 & 2.75116 \\ \mathrm{~h} & 4.67278 & -7.98205 & 1.71992 \\ \mathrm{n} & & & \end{array}$




$\begin{array}{lrrr}\text { h } & 3.23921 & -6.89625 & 1.53575 \\ \text { h } & 4.31864 & -7.20043 & 0.13256 \\ \text { h } & -3.24435 & -6.15811 & 1.86860 \\ \text { h } & -4.84073 & -4.28341 & 1.27323 \\ \text { h } & -3.90023 & -2.06286 & 0.55300 \\ \text { h } & -1.42439 & -1.71592 & 0.42298 \\ \text { h } & 2.10132 & -0.87060 & -0.23936\end{array}$

\section{$3 \mathrm{OH}-\mathrm{ZZZZ}$}

$E(B 3 L Y P /$ def2-TZVP $)=-1064.404075145$

35

$\begin{array}{llll}\text { C } & -3.51965 & -4.07017 & 1.67051\end{array}$

C $\quad-2.59291 \quad-3.50076 \quad 2.56096$

C $\quad-1.25289-3.35146 \quad 2.18161$

C $\quad-0.80439-3.78320 \quad 0.92046$

$\begin{array}{llll}\text { C } & -1.74056 & -4.37671 & 0.03816\end{array}$

c $\quad-3.08919-4.499950 .41096$

$\begin{array}{llll}0 & 0.53326 & -3.62430 & 0.66580\end{array}$

c $\quad 0.94371-2.98151-0.48163$

o $\quad 0.10902-2.42249-1.22100$

n $\quad-1.35459-4.95285-1.26948$

o $\quad-0.18523-5.31055-1.41336$

n $\quad 2.29058$-3.02833 -0.60809

c $\quad 2.81727-2.44433-1.68717$

o $\quad 2.10552-1.82609-2.61811$

C $\quad 4.26816-2.49829-1.85062$

C $\quad 4.92995-1.94365-2.91733$

o $4.36463-1.27349-3.92461$

c $\quad 5.23130-0.84349-4.98764$

$\begin{array}{llll}\text { C } & 4.42551 & 0.02675 & -5.93396\end{array}$

c $\quad 5.05770-3.21334-0.77966$

o $\quad-2.24045-5.07823-2.11293$

h $\quad 6.14532-3.20011-1.00652$

h $\quad 4.72804-4.27003-0.67930$

h $\quad 4.89854-2.74578 \quad 0.21633$

h $\quad 6.03441-2.04175-2.96718$

h $\quad 6.09291-0.28057-4.55590$

h $\quad 5.63465-1.74149-5.51238$

h $\quad 5.06569 \quad 0.36505 \quad-6.77688$

h $\quad 3.56455-0.53901-6.34927$

h $\quad 4.03122 \quad 0.92188-5.40721$

h $\quad-3.77960 \quad-4.94981-0.31694$

h $\quad-4.57700 \quad-4.18253 \quad 1.95735$

h $\quad-2.91699 \quad-3.15840 \quad 3.55704$

h $\quad-0.51306-2.88848 \quad 2.85222$

h $\quad \begin{array}{llll}1.12269 & -1.91197 & -2.29758\end{array}$

Transition state for the direct $2 \mathrm{NH}$ to Phenol Hydrogen Transfer $E(B 3 L Y P /$ def2-TZVP) $=-1064.356609029$ 
35

$\begin{array}{llll}\text { C } & -2.67858 & -4.72493 & 1.65387 \\ \text { C } & -2.79491 & -3.34388 & 1.78739 \\ \text { C } & -1.86933 & -2.44150 & 1.17610 \\ \text { C } & -0.81630 & -3.05148 & 0.40662 \\ \text { C } & -0.68666 & -4.45299 & 0.30863 \\ \text { C } & -1.61592 & -5.29473 & 0.91525 \\ \text { o } & -1.99371 & -1.15402 & 1.37525\end{array}$

$\begin{array}{llll}\text { C } & -3.38348 & 0.32796 & 0.49911\end{array}$

$\begin{array}{lllll}\mathrm{n} & -2.23386 & 0.81741 & 0.20593\end{array}$

c $\quad-2.08200 \quad 2.05962-0.57386$

$\begin{array}{llll}\text { C } & -0.71767 & 2.59521 & -0.54321\end{array}$

$\begin{array}{llll}\text { C } & 0.39051 & 1.99250 & 0.27954\end{array}$

n $\quad 0.17312 \quad-2.24656-0.29927$

o $\quad 1.22520-2.77970-0.65580$

$\begin{array}{lllll}0 & -4.52481 & 0.13793 & 0.63363\end{array}$

$\begin{array}{lllll}0 & -0.10030 & -1.05383 & -0.53817\end{array}$

$\begin{array}{lllll}0 & -3.06008 & 2.52412 & -1.13568\end{array}$

c $\quad-0.54434 \quad 3.71069-1.32232$

$\begin{array}{lllll}\text { o } & 0.61874 & 4.35824 & -1.39579\end{array}$

$\begin{array}{llll}\text { C } & 0.70628 & 5.46317 & -2.31896\end{array}$

c $\quad \begin{array}{llll}\text { c } & 1.97303 & 6.24057 & -2.01699\end{array}$

$\begin{array}{llll}\text { h } & -1.53903 & -0.05114 & 0.57537\end{array}$

$\begin{array}{llll}\text { h } & 1.28948 & 2.63982 & 0.23627\end{array}$

$\begin{array}{lllll}\text { h } & 0.66844 & 0.98009 & -0.08935\end{array}$

$\begin{array}{llll}\text { h } & 0.09367 & 1.88646 & 1.34680\end{array}$

h $\quad-1.39128 \quad 4.10993-1.91317$

h $\quad 0.71868 \quad 5.05634-3.35658$

h $\quad-0.19985 \quad 6.10267 \quad-2.20817$

$\begin{array}{llll}\text { h } & 2.07497 & 7.08614 & -2.73047\end{array}$

h $\quad \begin{array}{llll}1.95013 & 6.65198 & -0.98517\end{array}$

h $\quad 2.86834 \quad 5.59034-2.11604$

h $\quad 0.16024-4.84645-0.27203$

h $\quad-1.52168 \quad-6.387490 .81763$

$\begin{array}{llll}\text { h } & -3.42087 & -5.37967 & 2.14087\end{array}$

$\begin{array}{llll}\text { h } & -3.60581 & -2.89341 & 2.38149\end{array}$

Transition State for EEZE isomer $E(B 3 L Y P / d e f 2-T Z V P)=-1064.391075000$

35

\begin{tabular}{|c|c|c|}
\hline & $-2.30025 \quad-2.84137$ & 0.10643 \\
\hline c & $\begin{array}{ll}-1.83382 & -4.19399\end{array}$ & 0.06855 \\
\hline$c$ & $\begin{array}{ll}-1.70190 & -4.95334\end{array}$ & 1.24809 \\
\hline$C$ & $\begin{array}{ll}-2.03882 & -4.40698\end{array}$ & 2.48660 \\
\hline & $-2.51171-3.08009$ & 2.54853 \\
\hline & $-2.63969-2.32208$ & 1.38350 \\
\hline & $\begin{array}{lll}-1.46716 & -4.86378\end{array}$ & -1.18888 \\
\hline & $-0.93276-5.97167$ & -1.12071 \\
\hline & $-2.43989-2.03458$ & -0.94756 \\
\hline & $-3.82962 \quad-2.51348$ & -2.18590 \\
\hline & $\begin{array}{lll}-3.49138 & -2.02492\end{array}$ & -3.32933 \\
\hline
\end{tabular}




$\begin{array}{lrrr}\text { c } & -2.27823 & -1.58630 & -3.73352 \\ \text { c } & -2.09508 & -1.16642 & -5.11460 \\ \text { c } & -0.74773 & -0.65582 & -5.54801 \\ \text { o } & -4.68202 & -3.06824 & -1.58311 \\ \text { o } & -1.71400 & -4.28696 & -2.25716 \\ \text { o } & -1.24356 & -1.51283 & -2.95810 \\ \text { c } & -3.16842 & -1.27203 & -5.96138 \\ \text { o } & -3.10177 & -0.90820 & -7.24271 \\ \text { c } & -4.28268 & -1.11838 & -8.04538 \\ \text { c } & -4.02305 & -0.56133 & -9.43153 \\ \text { h } & -0.76063 & -0.37533 & -6.62004 \\ \text { h } & 0.03853 & -1.42636 & -5.38846 \\ \text { h } & -0.44475 & 0.23192 & -4.94981 \\ \text { h } & -4.13257 & -1.66910 & -5.59088 \\ \text { h } & -4.50035 & -2.21087 & -8.08200 \\ \text { h } & -5.14571 & -0.60884 & -7.55789 \\ \text { h } & -4.91665 & -0.71648 & -10.07305 \\ \text { h } & -3.80784 & 0.52775 & -9.38728 \\ \text { h } & -3.15809 & -1.07113 & -9.90705 \\ \text { h } & -1.32898 & -5.98263 & 1.15021 \\ \text { h } & -1.93786 & -5.01089 & 3.40206 \\ \text { h } & -2.78540 & -2.63604 & 3.52006 \\ \text { h } & -3.00771 & -1.28487 & 1.40915 \\ \text { h } & -1.57992 & -1.83460 & -1.95335\end{array}$

Transition State for EZZE isomer $E(B 3 L Y P /$ def2-TZVP) $=-1064.389778825$

35

\begin{tabular}{|c|c|c|c|}
\hline & -2.65169 & -2.32878 & 1.40474 \\
\hline & -2.29198 & -2.84221 & 0.13015 \\
\hline & -1.84399 & -4.20157 & 0.09173 \\
\hline & -1.75007 & -4.97274 & 1.26748 \\
\hline & -2.10738 & -4.43208 & 2.50259 \\
\hline & -2.56153 & -3.09845 & 2.56535 \\
\hline & -2.40457 & -2.02902 & -0.91983 \\
\hline & -1.45627 & -4.86510 & -1.16208 \\
\hline & -1.66948 & -4.27633 & -2.23154 \\
\hline & -0.93687 & -5.98007 & -1.09131 \\
\hline & -3.77942 & -2.52123 & -2.20923 \\
\hline & -4.63227 & -3.08931 & -1.62346 \\
\hline & -3.41859 & -2.01702 & -3.33878 \\
\hline & -2.20249 & -1.56789 & -3.71195 \\
\hline & -1.18343 & -1.49426 & -2.91171 \\
\hline & -2.06311 & -1.14936 & -5.10015 \\
\hline & -0.83899 & -0.66794 & -5.48678 \\
\hline & -0.59826 & -0.26123 & -6.73472 \\
\hline & 0.71623 & 0.26024 & -7.01610 \\
\hline & 0.79533 & 0.57804 & -8.49681 \\
\hline 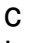 & -3.23479 & -1.25809 & -6.03928 \\
\hline & -2.95653 & -0.90780 & -7.05354 \\
\hline & -4.09637 & -0.65579 & -5.67667 \\
\hline & -3.59245 & 2.30844 & -6.1090 \\
\hline
\end{tabular}


Product Complex EEZE

$E(B 3 L Y P /$ def2-TZVP) $=-1064.420185736$

35

C $\quad \begin{array}{llll}0.74271 & 0.68150 & 3.07841\end{array}$

c $\quad 0.02169-0.49776 \quad 3.44406$

$\begin{array}{llll}\text { c } & -0.52755 & -0.65146 & 4.73499\end{array}$

C $\quad-0.37703 \quad 0.35280 \quad 5.68659$

C $\quad 0.33152 \quad 1.52727 \quad 5.34237$

C $\quad 0.87723 \quad 1.68694 \quad 4.07095$

$\begin{array}{llll}\text { n } & -0.17198 & -1.57886 & 2.49191\end{array}$

$\begin{array}{lllll}0 & -0.80798 & -2.57311 & 2.82774\end{array}$

$\begin{array}{llll}0 & 1.29561 & 0.91691 & 1.89010\end{array}$

$\begin{array}{lllll}\text { C } & -1.28967 & 0.55027 & -0.20390\end{array}$

$\begin{array}{lllll}\mathrm{n} & -0.69946 & 0.41519 & -1.26889\end{array}$

C $\quad 0.65771 \quad 0.17083-1.64497$

$\begin{array}{llll}\text { C } & 0.88708 & 0.02631 & -3.08665\end{array}$

c $\quad 2.30132-0.22363-3.53256$

$\begin{array}{llll}0 & -1.97165 & 0.70583 & 0.74434\end{array}$

$\begin{array}{llll}0 & 0.33002 & -1.44105 & 1.35114\end{array}$

$\begin{array}{lllll}0 & 1.55403 & 0.09554 & -0.81132\end{array}$

C $\quad-0.16851 \quad 0.11857 \quad-3.95108$

$\begin{array}{llll}0 & -0.02274 & -0.00836 & -5.27677\end{array}$

c $\quad-1.21192 \quad 0.11767-6.07756$

$\begin{array}{llll}\text { c } & -0.83059 & -0.07771 & -7.53275\end{array}$

h $\quad 2.36226-0.30624-4.63626$

h $\quad 2.69865-1.15833-3.07838$

$\begin{array}{lllll}\text { h } & 2.97275 & 0.59644 & -3.19414\end{array}$

h $\quad-1.192750 .30222-3.57456$

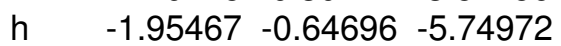

h $\quad-1.65832 \quad 1.12623 \quad-5.91010$

$\begin{array}{llll}\text { h } & -1.73151 & 0.01588 & -8.17605\end{array}$

$\begin{array}{lllll}\text { h } & -0.08961 & 0.68574 & -7.85288\end{array}$

h $\quad-0.38779-1.08404-7.69341$

h $\quad-1.07047 \quad-1.58330 \quad 4.94812$

$\begin{array}{llll}\text { h } & -0.80619 & 0.23183 & 6.69328\end{array}$

$\begin{array}{llll}\text { h } & 0.45743 & 2.33050 & 6.08712\end{array}$

h $\quad 1.43171 \quad 2.59521 \quad 3.78914$

$\begin{array}{llll}\text { h } & 1.16285 & 0.12479 & 1.27869\end{array}$ 
Product Complex EEZE

$E(B 3 L Y P /$ def2-TZVP $)=-1064.418481377$

35

$\begin{array}{llll}\text { C } & -2.18630 & -2.28419 & 1.85531\end{array}$

C $\quad-1.88460-2.816880 .57493$

$\begin{array}{llll}\text { C } & -1.88557 & -4.24099 & 0.44866\end{array}$

C $\quad-2.17406 \quad-5.07512 \quad 1.54983$

C $\quad-2.46554 \quad-4.52162 \quad 2.79305$

C $\quad-2.46994 \quad-3.11485 \quad 2.93682$

$\begin{array}{lllll}0 & -1.62645 & -1.95059 & -0.40347\end{array}$

n $\quad-1.58958-4.87730-0.82454$

o $\quad-1.32173-4.13111-1.79586$

$\begin{array}{llll}0 & -1.60884 & -6.10161 & -0.90603\end{array}$

$\begin{array}{llll}\text { C } & -3.85460 & -2.80334 & -2.73237\end{array}$

o $\quad-4.44068-3.34761-1.86760$

n $\quad-3.36714 \quad-2.24822-3.70925$

C $\quad-2.11367-1.64912-4.02241$

o $\quad-1.21995-1.55270-3.18521$

$\begin{array}{llll}\text { c } & -2.04165 & -1.18036 & -5.41657\end{array}$

$\begin{array}{llll}\text { c } & -0.85574 & -0.60362 & -5.77875\end{array}$

$\begin{array}{lllll}0 & -0.62938 & -0.12727 & -7.00880\end{array}$

$\begin{array}{lllll}\text { C } & 0.67053 & 0.43948 & -7.25660\end{array}$

$\begin{array}{llll}\text { c } & 0.71817 & 0.91415 & -8.69649\end{array}$

c $\quad-3.20291-1.33988-6.36093$

$\begin{array}{llll}\text { h } & -2.95213 & -0.92917 & -7.36034\end{array}$

h $\quad-4.10851 \quad-0.81557-5.98272$

h $\quad-3.48266-2.41001-6.48146$

h $\quad-0.03729-0.51843 \quad-5.03821$

h $\quad 0.84016 \quad 1.28300 \quad-6.54729$

h $\quad 1.44781-0.33574-7.06152$

h $\quad \begin{array}{llll}1.71326 & 1.35678 & -8.91633\end{array}$

h $\quad 0.54793 \quad 0.06885-9.39715$

h $\quad-0.05853 \quad 1.68604-8.88437$

h $\quad-2.15903 \quad-6.16137 \quad 1.38142$

h $\quad-2.69023 \quad-5.17360 \quad 3.65129$

h $\quad-2.69938-2.66424 \quad 3.91659$

h $\quad-2.18531-1.18763 \quad 1.95306$

h $\quad-1.39757-2.44238-1.25510$

Molecular energies (in a.u.) and molecular structures of 7c (Table S3)

2NH-EEEE

$E(B 3 L Y P /$ def2-TZVP) $=-1025.117771759$

32

C $\quad-1.48823 \quad-3.93508 \quad 0.56892$

$\begin{array}{llll}\text { C } & -0.92672 & -5.22971 & 0.63009\end{array}$

$\begin{array}{llll}\text { C } & -1.07180 & -6.00278 & 1.78327\end{array}$

C $\quad-1.77902 \quad-5.46901 \quad 2.87153$

C $\quad-2.33405-4.18375 \quad 2.82358$

http://mc.manuscriptcentral.com/poc 


\section{NH-EEEZ}

$E(B 3 L Y P /$ def2-TZVP $)=-1025.124000521$

32

\begin{tabular}{|c|c|c|c|}
\hline & -1.99519 & -3.57996 & 0.42000 \\
\hline C & -2.22829 & -4.95992 & 0.39625 \\
\hline & -2.02763 & -5.71687 & 1.55899 \\
\hline & -1.59167 & -5.12738 & 2.75479 \\
\hline & -1.36242 & -3.74927 & 2.78230 \\
\hline & -1.56514 & -2.97837 & 1.62027 \\
\hline & -2.27799 & -7.17691 & 1.52342 \\
\hline & -2.08264 & -7.81002 & 2.56150 \\
\hline & -1.24954 & -1.63997 & 1.73733 \\
\hline & -1.95736 & -0.65470 & 1.05125 \\
\hline & -2.92375 & -0.86911 & 0.34962 \\
\hline & -1.35636 & 0.54647 & 1.35697 \\
\hline & -1.41987 & 1.85739 & 0.75671 \\
\hline & -0.42031 & 2.55216 & 0.91179 \\
\hline & -2.60133 & 2.33845 & 0.04779 \\
\hline & -3.87229 & 1.84810 & 0.08804 \\
\hline & -4.86963 & 2.47635 & -0.54412 \\
\hline & -6.17307 & 1.86760 & -0.46031 \\
\hline & -7.14947 & 2.72323 & -1.24462 \\
\hline & -2.42189 & 3.28412 & -0.48822 \\
\hline & -2.66475 & -7.66013 & 0.45906 \\
\hline
\end{tabular}




$\begin{array}{lrrr}\text { h } & -2.14980 & -2.97517 & -0.48227 \\ \text { h } & -0.49428 & 0.46418 & 1.90901 \\ \text { h } & -4.13763 & 0.92123 & 0.62487 \\ \text { h } & -6.11795 & 0.83387 & -0.87356 \\ \text { h } & -6.47181 & 1.79538 & 0.61179 \\ \text { h } & -8.16628 & 2.27790 & -1.19646 \\ \text { h } & -7.19632 & 3.75236 & -0.82849 \\ \text { h } & -6.84646 & 2.79110 & -2.31134 \\ \text { h } & -2.56284 & -5.46762 & -0.51973 \\ \text { h } & -1.44359 & -5.75836 & 3.64264 \\ \text { h } & -1.02093 & -3.24707 & 3.70030\end{array}$

2NH-EEZE
$E(B 3 L Y P /$ def2-TZVP $)=-1025.120976310$

32

C $\quad-3.10801 \quad-4.451690 .54669$

$\begin{array}{llll}\text { c } & -2.80628 & -5.05098 & 1.77651\end{array}$

$\begin{array}{llll}\text { C } & -1.70544 & -4.60485 & 2.51889\end{array}$

$\begin{array}{llll}\text { C } & -0.88495 & -3.56084 & 2.06361\end{array}$

$\begin{array}{llll}\text { C } & -1.17996 & -2.95927 & 0.83985\end{array}$

$\begin{array}{llll}\text { c } & -2.28542 & -3.40388 & 0.08411\end{array}$

n $\quad-1.39820 \quad-5.25072 \quad 3.81560$

$\begin{array}{lllll}0 & -0.42479 & -4.82981 & 4.44215\end{array}$

$\begin{array}{llll}0 & -2.42916 & -2.73280 & -1.11222\end{array}$

C $\quad-3.52088-2.79857-1.92176$

o $\quad-4.50161 \quad-3.50135-1.77134$

n $\quad-3.36721 \quad-1.95231-3.01679$

c $\quad-2.42385-0.90527-3.26542$

$\begin{array}{lllll}0 & -1.66227 & -0.47906 & -2.41224\end{array}$

$\begin{array}{llll}\text { c } & -2.45754 & -0.37256 & -4.64204\end{array}$

$\begin{array}{llll}\text { c } & -2.91910 & -1.03441 & -5.73737\end{array}$

$\begin{array}{lllll}0 & -2.87221 & -0.50356 & -6.96628\end{array}$

$\begin{array}{llll}\text { c } & -3.24678 & -1.35520 & -8.06421\end{array}$

$\begin{array}{llll}\text { c } & -3.29709 & -0.51473 & -9.32557\end{array}$

$\begin{array}{lllll}\text { h } & -1.96613 & 0.60504 & -4.77034\end{array}$

$\begin{array}{llll}\text { o } & -2.13487 & -6.16673 & 4.18316\end{array}$

h $\quad-3.96780-4.78202-0.04766$

h $\quad-4.16259-2.02652-3.65628$

h $\quad-3.34084-2.05920-5.67311$

h $\quad-2.49745-2.17608-8.15810$

h $\quad-4.23938-1.81726-7.84813$

h $\quad-3.57588-1.15043-10.19291$

h $\quad-4.04731 \quad 0.29909-9.22903$

h $\quad-2.30720 \quad-0.05484-9.53199$

h $\quad-3.41956-5.87225 \quad 2.17456$

h $\quad-0.03213 \quad-3.23923 \quad 2.67825$

h $\quad-0.57302 \quad-2.13393 \quad 0.43687$

2NH-EEZZ

$E(B 3 L Y P /$ def2-TZVP) $=-1025.119671555$

http://mc.manuscriptcentral.com/poc 
$\begin{array}{llll}\text { C } & -3.17602 & -2.84545 & 1.23462\end{array}$

c $\quad-1.84466 \quad-2.591150 .88534$

C $\quad-0.97293 \quad-3.679120 .67176$

$\begin{array}{llll}\text { c } & -1.43014 & -5.00598 & 0.80697\end{array}$

C $\quad-2.75860 \quad-5.25669 \quad 1.15763$

$\begin{array}{llll}\text { C } & -3.61915 & -4.16872 & 1.36601\end{array}$

$\begin{array}{lllll}\text { o } & 0.36921 & -3.54722 & 0.38644\end{array}$

c $\quad 0.86822-2.46813-0.35932$

n $\quad 2.24343-2.63389-0.38781$

c $\quad 3.18783-1.69592-0.90862$

$\begin{array}{llll}\text { C } & 4.57439 & -2.19623 & -0.99207\end{array}$

h $\quad 5.33378-1.41174-1.13778$

n $\quad-5.02959-4.42705 \quad 1.73717$

$\begin{array}{lllll}0 & -5.38292 & -5.60290 & 1.83662\end{array}$

o $\quad 0.20319-1.60578-0.87153$

o $\quad 2.86664-0.57019-1.24598$

C $\quad 4.95718-3.50116-1.01174$

o $\quad 6.24115-3.86741-1.12639$

c $\quad 6.51296-5.27203-1.27835$

C $\quad 8.01575-5.47399-1.23917$

$\begin{array}{lllll}0 & -5.75571 & -3.45015 & 1.92268\end{array}$

h $\quad-0.72244 \quad-5.83098 \quad 0.63314$

h $\quad 2.59589-3.44984 \quad 0.11917$

h $\quad 4.22632-4.33506-0.95389$

h $\quad 6.00945-5.83422-0.45605$

h $\quad 6.08603 \quad-5.62211-2.24759$

h $\quad 8.25614 \quad-6.55187-1.35985$

h $\quad 8.51008-4.91046-2.05895$

h $\quad 8.43610-5.12512-0.27181$

h $\quad-3.14751 \quad-6.27849 \quad 1.27096$

h $\quad-3.88625 \quad-2.02528 \quad 1.41230$

$\begin{array}{llll}\text { h } & -1.48747 & -1.56015 & 0.77197\end{array}$

\section{NH-EZEE}

$E(B 3 L Y P /$ def2-TZVP $)=-1025.126778419$

32

$\begin{array}{lrrr}\text { c } & -1.37286 & -3.61373 & 1.71872 \\ \text { C } & -2.22907 & -4.39028 & 2.52456 \\ \text { C } & -3.61319 & -4.21955 & 2.40439 \\ \text { C } & -4.12028 & -3.28712 & 1.48892 \\ \text { C } & -3.27417 & -2.51372 & 0.68118 \\ \text { C } & -1.89190 & -2.67955 & 0.80103 \\ \text { n } & -5.58737 & -3.11583 & 1.36787 \\ \text { o } & -6.30046 & -3.80805 & 2.09497 \\ \text { o } & 0.00376 & -3.74591 & 1.70317 \\ \text { c } & 0.71889 & -4.05645 & 2.84017 \\ \text { n } & 2.06942 & -4.20447 & 2.57191 \\ \text { C } & 2.94423 & -4.13637 & 1.44228 \\ \text { C } & 2.38357 & -3.96404 & 0.09958 \\ \text { h } & 1.30206 & -3.89807 & -0.06962\end{array}$


$\begin{array}{llll}0 & 0.24379 & -4.17197 & 3.95023\end{array}$

$\begin{array}{lllll}0 & 4.14491 & -4.24838 & 1.68549\end{array}$

C $\quad 3.25352-3.89739-0.94624$

o $\quad 2.84758-3.75245-2.20996$

C $\quad 3.87412-3.69143-3.22036$

C $\quad 3.20362-3.53866-4.57202$

$\begin{array}{llll}0 & -5.99602 & -2.29379 & 0.54757\end{array}$

$\begin{array}{llll}\text { h } & -1.81836 & -5.11151 & 3.24204\end{array}$

h $\quad 2.60799 \quad-4.37896 \quad 3.42839$

h $\quad 4.34427 \quad-3.96557 \quad-0.76194$

h $\quad 4.54570-2.82870-3.00260$

h $\quad 4.48349-4.62396-3.17467$

h $\quad 3.97300-3.48647-5.37200$

h $\quad 2.53639-4.40160-4.78246$

h $\quad 2.59610-2.60926-4.60923$

h $\quad-4.31570 \quad-4.80583 \quad 3.01400$

h $\quad-3.71368-1.79391-0.02376$

h $\quad-1.19351 \quad-2.08870 \quad 0.18874$

2NH-EZEZ

$E(B 3 L Y P / d e f 2-T Z V P)=-1025.131767374$

32

$\begin{array}{llll}\text { C } & -1.31149 & -3.25328 & 0.23412\end{array}$

$\begin{array}{llll}\text { C } & -1.90491 & -4.44472 & -0.23247\end{array}$

$\begin{array}{llll}\text { C } & -2.55445 & -5.28269 & 0.68205\end{array}$

$\begin{array}{llll}\text { C } & -2.60269 & -4.92930 & 2.03734\end{array}$

$\begin{array}{llll}\text { C } & -2.01082 & -3.74843 & 2.50991\end{array}$

C $\quad-1.36454-2.90848 \quad 1.60042$

$\begin{array}{llll}\text { n } & -3.29082 & -5.82527 & 2.99536\end{array}$

$\begin{array}{lllll}0 & -3.79804 & -6.85371 & 2.54671\end{array}$

o $\quad-0.59625-2.37463-0.55340$

C $\quad-0.83109-2.21520-1.91544$

n $\quad 0.10184-1.30933-2.37442$

c $\quad 0.33238-0.77760 \quad-3.68052$

C $\quad-0.53363-1.23600-4.76757$

h $\quad-1.33625-1.96115-4.57485$

o $\quad-1.70402-2.77516-2.54288$

$\begin{array}{lllll}0 & 1.25213 & 0.03075 & -3.78932\end{array}$

$\begin{array}{llll}\text { C } & -0.30960 & -0.73518 & -6.01408\end{array}$

o $\quad-1.03865-1.09243-7.07368$

C $\quad-0.70945-0.47285-8.33202$

c $\quad-1.61766-1.05238-9.39958$

$\begin{array}{llll}\text { o } & -3.30905 & -5.48236 & 4.17816\end{array}$

h $\quad-1.86698-4.70682-1.29642$

h $\quad 0.75771-0.93234-1.68164$

h $\quad 0.50608-0.00242-6.17818$

$\begin{array}{lllll}\text { h } & -0.84733 & 0.62975 & -8.23987\end{array}$

h $\quad 0.36336-0.66855-8.56422$

h $\quad-1.38919-0.58908-10.38324$

h $\quad-1.47448-2.15070-9.48675$

h $\quad-2.68459-0.85720-9.15882$

h $\quad-3.02892-6.22006 \quad 0.35790$ 
h $\quad-2.06990 \quad-3.50849 \quad 3.58096$

h $\quad-0.88712 \quad-1.97302 \quad 1.92977$

6
7

8

\author{
2NH-EZZE \\ $E(B 3 L Y P /$ def2-TZVP $)=-1025.126351542$
}

32

$\begin{array}{llll}\text { C } & -2.11187 & -4.18933 & 0.09330\end{array}$

C $\quad-3.29204 \quad-4.68744 \quad 0.68410$

$\begin{array}{llll}\text { C } & -3.39158 & -4.69939 & 2.08171\end{array}$

$\begin{array}{llll}\text { C } & -2.33002 & -4.22413 & 2.86258\end{array}$

$\begin{array}{llll}\text { C } & -1.15326 & -3.72789 & 2.27918\end{array}$

$\begin{array}{llll}\text { c } & -1.04770 & -3.71150 & 0.88817\end{array}$

$\begin{array}{lllll}\mathrm{n} & -2.45084 & -4.24511 & 4.33794\end{array}$

o $\quad-3.49963 \quad-4.67873 \quad 4.81654$

o $\quad-1.85400-4.10155-1.25805$

C $\quad-2.69194 \quad-4.51232-2.24774$

n $\quad-2.13232 \quad-4.27140-3.49962$

c $\quad-0.89474-3.68245-3.89011$

C $\quad-0.74326-3.63587-5.35820$

h $\quad-1.52214 \quad-4.02571-6.03475$

o $\quad-3.78626-5.02537-2.11333$

$\begin{array}{llll}0 & -0.06549 & -3.26751 & -3.09199\end{array}$

C $\quad 0.39234-3.09445-5.87520$

o $\quad 0.62633-3.00555-7.18659$

C $\quad 1.87302-2.40002-7.58430$

c $\quad 1.94377-2.40647-9.09906$

$\begin{array}{llll}0 & -1.49355 & -3.82724 & 4.99129\end{array}$

h $\quad-4.11027 \quad-5.05690 \quad 0.05538$

h $\quad-2.75786 \quad-4.58157 \quad-4.24844$

h $\quad 1.16793-2.70555-5.18484$

h $\quad 1.91536-1.36099-7.18206$

h $\quad 2.71545-2.97790-7.13776$

h $\quad 2.89645-1.94400-9.43498$

h $\quad 1.90017-3.44450-9.49280$

h $\quad 1.10066-1.83033-9.53673$

h $\quad-4.29380-5.07791 \quad 2.58335$

h $\quad-0.34367 \quad-3.36399 \quad 2.92752$

$\begin{array}{llll}\text { h } & -0.14942 & -3.33187 & 0.37777\end{array}$

2NH-EZZZ

$E(B 3 L Y P /$ def2-TZVP) $=-1025.125452800$

32

$\begin{array}{llll}\text { C } & -3.48864 & -4.42923 & 1.66061\end{array}$

C $\quad-3.08653-3.11169 \quad 1.40203$

$\begin{array}{llll}\text { C } & -1.84323 & -2.86775 & 0.80645\end{array}$

$\begin{array}{llll}\text { c } & -1.01641 & -3.96224 & 0.47576\end{array}$

C $\quad-1.43226-5.28406 \quad 0.74131$

$\begin{array}{llll}\text { C } & -2.67204 & -5.52310 & 1.33743\end{array}$ 
$\begin{array}{llll}0 & 0.24775 & -3.85260 & -0.06091\end{array}$

c $\quad 0.64567-2.76340-0.84930$

o $\quad-0.05749-1.84606-1.18634$

$\begin{array}{llll}\text { n } & -4.80380 & -4.67420 & 2.29475\end{array}$

$\begin{array}{llll}\text { o } & -5.12236 & -5.84533 & 2.50528\end{array}$

n $\quad 1.98024-2.98425-1.14761$

c $\quad 2.81813-2.14367-1.93519$

o $2.41685-1.10749-2.44212$

C $\quad 4.19091-2.67283-2.05537$

C $\quad 5.09303-1.96831-2.79035$

$\begin{array}{lllll}\text { o } & 6.36054 & -2.35410 & -2.95451\end{array}$

C $\quad 7.19039-1.52325-3.79142$

c $\quad 8.60334-2.07293-3.75677$

h $\quad 4.49496-3.61408-1.56718$

$\begin{array}{lllll}0 & -5.49234 & -3.69147 & 2.57195\end{array}$

$\begin{array}{llll}\text { h } & -0.76134 & -6.11400 & 0.47157\end{array}$

h $\quad 2.38381-3.84098-0.75916$

h $\quad 4.77259-1.02527-3.27775$

h $\quad 6.77903-1.53050-4.82764$

h $\quad 7.15472 \quad-0.47524-3.41307$

h $\quad 9.26525-1.45444-4.39992$

h $\quad 9.00793-2.06048-2.72200$

h $\quad 8.62973-3.11888-4.13038$

h $\quad-3.02471 \quad-6.54076 \quad 1.55689$

h $\quad-3.75874-2.28596 \quad 1.67606$

$\begin{array}{llll}\text { h } & -1.52144 & -1.84172 & 0.59142\end{array}$

2NH-ZEEE

$E(B 3 L Y P /$ def2-TZVP $)=-1025.116673190$

32

$\begin{array}{llll}\text { C } & -0.85915 & -5.10996 & 0.65531\end{array}$

C $\quad-1.50803-5.90445 \quad 1.60181$

$\begin{array}{llll}\text { C } & -2.46140 & -5.31178 & 2.44410\end{array}$

$\begin{array}{llll}\text { C } & -2.76443 & -3.94644 & 2.35903\end{array}$

C $\quad-2.11423-3.14246 \quad 1.41346$

C $\quad-1.16284-3.733690 .55320$

n $\quad-3.15963 \quad-6.14926 \quad 3.44613$

$\begin{array}{llll}0 & -3.98735 & -5.59649 & 4.17096\end{array}$

$0 \quad-0.45022-3.08485-0.42750$

C $\quad-0.68194-1.79376-0.84182$

$\begin{array}{lllll}0 & -1.32080 & -0.95521 & -0.23983\end{array}$

n $\quad-0.00313 \quad-1.54657-2.02285$

c $\quad 0.64419-2.38836-2.97503$

o $\quad 1.57254-1.91639-3.62055$

C $\quad 0.12900-3.74568-3.23008$

C $\quad-1.14792-4.20843-3.12450$

$\begin{array}{lllll}0 & -2.16985 & -3.50821 & -2.61126\end{array}$

c $\quad-3.44180-4.17660-2.49559$

$\begin{array}{llll}\text { c } & -4.48344 & -3.15071 & -2.09192\end{array}$

h $\quad 0.84672 \quad-4.39180-3.75761$

$\begin{array}{lllll}\text { o } & -2.86775 & -7.34538 & 3.48542\end{array}$

h $\quad-0.10318-5.53304 \quad-0.02379$

http://mc.manuscriptcentral.com/poc 
h $\quad 0.17279-0.54936-2.18819$

h $\quad-1.39872-5.22138-3.49941$

h $\quad-3.69400 \quad-4.64836-3.47419$

h $\quad-3.35306-4.98342-1.73091$

h $\quad-5.47256-3.64325-1.97638$

h $\quad-4.21321-2.67493-1.12475$

h $\quad-4.57394-2.35392-2.86076$

h $\quad-1.29318-6.97754 \quad 1.70473$

h $\quad-3.51081-3.52343 \quad 3.04690$

h $\quad-2.32571 \quad-2.06915 \quad 1.34201$

\section{NH-ZEEZ}

$E(B 3 L Y P /$ def2-TZVP $)=-1025.120799869$

32

$\begin{array}{llll}\text { C } & -2.51381 & -5.50616 & 1.30741\end{array}$

$\begin{array}{llll}\text { C } & -2.37872 & -4.94386 & 2.58466\end{array}$

$\begin{array}{llll}\text { C } & -1.72459 & -3.71928 & 2.78421\end{array}$

C $\quad-1.20330-3.04596 \quad 1.67649$

c $\quad-1.33537-3.60051 \quad 0.38725$

$\begin{array}{llll}\text { C } & -1.99002 & -4.83489 & 0.19665\end{array}$

$\begin{array}{lllll}\mathrm{n} & -2.93700 & -5.66237 & 3.75367\end{array}$

$\begin{array}{lllll}0 & -2.79580 & -5.13982 & 4.85988\end{array}$

$\begin{array}{lllll}0 & -0.73095 & -2.88832 & -0.62588\end{array}$

C $\quad-1.26891-2.84566-1.91532$

$\begin{array}{lllll}n & -0.36767 & -2.14404 & -2.68971\end{array}$

c $\quad-0.36383-1.89588-4.09648$

$\begin{array}{llll}\text { C } & -1.63411 & -1.79214 & -4.82644\end{array}$

h $\quad-1.53864 \quad-1.98397-5.90596$

o $\quad-2.32466-3.33945-2.23785$

$\begin{array}{lllll}0 & 0.72705 & -1.75648 & -4.63759\end{array}$

C $\quad-2.82915-1.31081-4.38700$

$\begin{array}{llll}\text { o } & -3.04900 & -0.84466 & -3.15355\end{array}$

$\begin{array}{llll}\text { C } & -4.41864 & -0.75079 & -2.72363\end{array}$

$\begin{array}{lllll}\text { C } & -4.46700 & 0.06195 & -1.44371\end{array}$

$\begin{array}{lllll}\text { o } & -3.50537 & -6.73410 & 3.54246\end{array}$

h $\quad-2.09123-5.26255-0.80884$

h $\quad 0.55549 \quad-1.98913-2.26864$

h $\quad-3.68813-1.24374-5.08711$

h $\quad-5.02163 \quad-0.27293-3.53293$

h $\quad-4.80512-1.78205-2.55961$

$\begin{array}{lllll}\text { h } & -5.51680 & 0.14768 & -1.08996\end{array}$

h $\quad-3.87085-0.42885-0.64465$

h $\quad-4.06491 \quad 1.08521 \quad-1.60571$

h $\quad-3.02800 \quad-6.47218 \quad 1.20226$

h $\quad-1.63845 \quad-3.31556 \quad 3.80297$

h $\quad-0.68201 \quad-2.08293 \quad 1.78799$

2NH-ZEZE

$E(B 3 L Y P /$ def2-TZVP $)=-1025.125109230$

http://mc.manuscriptcentral.com/poc 
32

$\begin{array}{llll}\text { c } & -3.30034 & -4.85218 & 1.86383 \\ \text { c } & -2.38473 & -4.39854 & 2.82240 \\ \text { c } & -1.30238 & -3.57518 & 2.47251 \\ \text { c } & -1.14404 & -3.20407 & 1.13715 \\ \text { c } & -2.06283 & -3.65446 & 0.16415 \\ \text { c } & -3.14784 & -4.48314 & 0.52076 \\ \text { h } & -2.56223 & -4.79511 & 4.23728 \\ \text { o } & -1.73817 & -4.37539 & 5.05140 \\ \text { o } & -1.76902 & -3.19950 & -1.10235 \\ \text { c } & -2.49496 & -3.46289 & -2.22466 \\ \text { h } & -1.93672 & -2.84324 & -3.33572 \\ \text { c } & -0.78721 & -2.02267 & -3.47589 \\ \text { c } & -0.53674 & -1.55659 & -4.86337 \\ \text { h } & 0.36334 & -0.93007 & -4.95036 \\ \text { o } & -3.50224 & -4.14211 & -2.29624 \\ \text { o } & -0.04886 & -1.71369 & -2.55248 \\ \text { c } & -1.24300 & -1.78520 & -6.00283 \\ \text { o } & -2.38011 & -2.51228 & -6.05329 \\ \text { c } & -2.95715 & -2.77113 & -7.34921 \\ \text { c } & -4.31560 & -3.41533 & -7.15037 \\ \text { o } & -3.52180 & -5.51787 & 4.50992 \\ \text { h } & -3.85365 & -4.82609 & -0.24442 \\ \text { h } & -2.47452 & -3.02573 & -4.19356 \\ \text { h } & -0.90793 & -1.35899 & -6.96873 \\ \text { h } & -3.04223 & -1.80665 & -7.90165 \\ \text { h } & -2.27080 & -3.43997 & -7.91864 \\ \text { h } & -4.77515 & -3.63441 & -8.13782 \\ \text { h } & -4.22558 & -4.37024 & -6.58936 \\ \text { h } & -4.99698 & -2.74122 & -6.58860 \\ \text { h } & -4.13158 & -5.49625 & 2.18495 \\ \text { h } & -0.60611 & -3.24093 & 3.25457 \\ \text { h } & -0.31477 & -2.55996 & 0.80712 \\ & & & \end{array}$

2NH-ZEZZ

$E(B 3 L Y P /$ def2-TZVP $)=-1025.123573841$

32

$\begin{array}{llll}\text { C } & -3.58565 & -4.37659 & 1.22668\end{array}$

c $\quad-3.19962 \quad-3.03316 \quad 1.12840$

$\begin{array}{llll}\text { c } & -1.84984 & -2.70367 & 0.95278\end{array}$

$\begin{array}{llll}\text { c } & -0.89622 & -3.74250 & 0.87730\end{array}$

C $\quad-1.29904 \quad-5.09272 \quad 0.97714$

C $\quad-2.64508 \quad-5.41549 \quad 1.15382$

$\begin{array}{llll}0 & 0.46277 & -3.57102 & 0.74698\end{array}$

$\begin{array}{llll}\text { C } & 1.06734 & -2.37087 & 0.34082\end{array}$

$\begin{array}{lllll}0 & 0.48774 & -1.36401 & 0.02184\end{array}$

$\begin{array}{llll}\text { n } & -5.01525 & -4.70975 & 1.41364\end{array}$

$\begin{array}{llll}\text { o } & -5.31260 & -5.90239 & 1.50238\end{array}$

$\begin{array}{llll}\text { n } & 2.42884 & -2.61046 & 0.38527\end{array}$

c $\quad 3.44903-1.67386 \quad 0.07830$

$\begin{array}{llll}\text { o } & 3.22151 & -0.52634 & -0.26666\end{array}$

http://mc.manuscriptcentral.com/poc 
2NH-ZZEE

$E(B 3 L Y P / d e f 2-T Z V P)=-1025.120527187$

\section{2}

$\begin{array}{llll}\text { C } & -2.73489 & -4.48030 & 2.03251\end{array}$

C $\quad-4.11801 \quad-4.41946 \quad 1.82552$

$\begin{array}{llll}\text { C } & -4.67828 & -3.29350 & 1.20610\end{array}$

C $\quad-3.88656 \quad-2.21610 \quad 0.78285$

$\begin{array}{llll}\text { c } & -2.50590 & -2.27309 & 0.98991\end{array}$

C $\quad-1.93324-3.40049 \quad 1.61111$

$\begin{array}{llll}\mathrm{n} & -6.14323 & -3.24045 & 0.98926\end{array}$

$\begin{array}{lllll}0 & -6.59886 & -2.23378 & 0.44547\end{array}$

$\begin{array}{lllll}0 & -0.55410 & -3.38227 & 1.69869\end{array}$

$\begin{array}{llll}\text { C } & 0.11645 & -3.96997 & 2.75412\end{array}$

$\begin{array}{lllll}0 & -0.42010 & -4.47771 & 3.71669\end{array}$

n $\quad 1.48754-3.88965 \quad 2.59558$

$\begin{array}{llll}\text { c } \quad 2.43087 & -3.35317 & 1.64960\end{array}$

$\begin{array}{llll}0 & 3.60204 & -3.34241 & 2.01062\end{array}$

c $\quad 1.94318-2.909820 .34202$

$\begin{array}{llll}\text { C } & 2.74427 & -2.36174 & -0.61960\end{array}$

$\begin{array}{lllll}0 & 4.04715 & -2.12126 & -0.48924\end{array}$

C $\quad 4.73788-1.59977-1.63973$

c $\quad 6.14842-1.23386-1.21958$

h $\quad 0.88295-3.02714 \quad 0.08971$

$\begin{array}{llll}\text { o } & -6.80745 & -4.20651 & 1.36517\end{array}$

h $\quad-2.28361 \quad-5.35122 \quad 2.52389$

h $\quad \begin{array}{llll}1.98392 & -4.24674 & 3.42041\end{array}$

h $\quad 2.29545-2.07670-1.59426$

h $\quad 4.74533-2.37805-2.43889$

h $\quad 4.18696-0.71051-2.02788$

h $\quad 6.71450 \quad-0.84208-2.09178$

h $\quad 6.13423-0.45498-0.42758$

h $\quad 6.68126-2.12337-0.82151$

h $\quad-4.77897 \quad-5.24069 \quad 2.13765$ 


$$
\begin{array}{llll}
\text { h } & -4.36670 & -1.35176 & 0.30263 \\
\text { h } & -1.84877 & -1.44700 & 0.67777
\end{array}
$$

\section{NH-ZZEZ}

$E(B 3 L Y P /$ def2-TZVP $)=-1025.126382228$

32

$\begin{array}{llll}\text { C } & -1.89390 & -4.59113 & -0.00297\end{array}$

$\begin{array}{llll}\text { C } & -2.57287 & -5.26666 & 1.01759\end{array}$

C $\quad-2.81778-4.61828 \quad 2.23580$

$\begin{array}{llll}\text { C } & -2.39525 & -3.30069 & 2.46543\end{array}$

C $\quad-1.71957-2.62322 \quad 1.44786$

$\begin{array}{llll}\text { C } & -1.46985 & -3.26467 & 0.21783\end{array}$

$\begin{array}{lllll}\mathrm{n} & -3.53683 & -5.34311 & 3.30893\end{array}$

$\begin{array}{lllll}0 & -3.73250 & -4.74184 & 4.36592\end{array}$

$\begin{array}{llll}0 & -0.74031 & -2.52505 & -0.68918\end{array}$

c $\quad-0.90146-2.66477-2.06620$

$\begin{array}{llll}\text { o } & -1.72491 & -3.38031 & -2.59657\end{array}$

n $\quad 0.02679-1.83991-2.66090$

c $\quad 0.31549-1.56636-4.04202$

$\begin{array}{lllll}0 & 1.22334 & -0.77348 & -4.26174\end{array}$

$\begin{array}{llll}\text { C } & -0.50054 & -2.25797 & -5.03971\end{array}$

c $\quad-0.34546-2.10594-6.38799$

o $\quad 0.56022-1.32237-6.97206$

c $\quad 0.55995-1.28208-8.41093$

c $\quad 1.69272-0.37831-8.85891$

h $\quad-1.28812-2.94195-4.69411$

$\begin{array}{llll}0 & -3.89119 & -6.49876 & 3.07436\end{array}$

h $\quad-1.70129-5.08561-0.96313$

h $\quad 0.63788-1.30793-2.03157$

h $\quad-1.01235-2.66921-7.07353$

h $\quad 0.68935-2.31748-8.80572$

h $\quad-0.42609-0.89814-8.76395$

h $\quad 1.71757 \quad-0.32254-9.96825$

h $\quad \begin{array}{llll}1.55891 & 0.64792 & -8.45552\end{array}$

h $\quad 2.67004-0.76652 \quad-8.50133$

$\begin{array}{llll}\text { h } & -2.91808 & -6.30213 & 0.88567\end{array}$

h $\quad-2.60653 \quad-2.82940 \quad 3.43585$

h $\quad-1.37141 \quad-1.58821 \quad 1.58659$

2NH-ZZZE

$E(B 3 L Y P /$ def2-TZVP $)=-1025.120119075$

32

$\begin{array}{llll}\text { C } & -3.39944 & -4.59889 & 0.63648\end{array}$

$\begin{array}{llll}\text { C } & -3.51028 & -4.65423 & 2.03217\end{array}$

$\begin{array}{llll}\text { c } & -2.41559 & -4.30836 & 2.83510\end{array}$

$\begin{array}{llll}\text { C } & -1.19361 & -3.90127 & 2.27590\end{array}$

$\begin{array}{llll}\text { C } & -1.07607 & -3.84312 & 0.88708\end{array}$

$\begin{array}{llll}\text { C } & -2.17381 & -4.19001 & 0.06968\end{array}$

http://mc.manuscriptcentral.com/poc 
32

\begin{tabular}{|c|c|c|c|}
\hline C & -2.78892 & -5.34281 & 1.46462 \\
\hline C & -3.55122 & -4.21526 & 1.80478 \\
\hline & -3.10734 & -2.91692 & 1.51874 \\
\hline & -1.87765 & -2.72677 & 0.87694 \\
\hline & -1.10617 & -3.85552 & 0.52657 \\
\hline & -1.56350 & -5.15754 & 0.82187 \\
\hline & -4.85246 & -4.40325 & 2.48475 \\
\hline & -5.49500 & -3.39313 & 2.77249 \\
\hline & 0.13824 & -3.80224 & -0.06064 \\
\hline & 0.55119 & -2.73189 & -0.87124 \\
\hline & 1.85440 & -3.01761 & -1.23622 \\
\hline & 2.69364 & -2.20746 & -2.06825 \\
\hline & 4.02087 & -2.82166 & -2.26521 \\
\hline 1 & 4.25475 & -3.78682 & -1.78625 \\
\hline & -0.12382 & -1.78135 & -1.17278 \\
\hline & 2.31698 & -1.14799 & -2.53506 \\
\hline & 5.01833 & -2.27669 & -3.02146 \\
\hline & 4.92361 & -1.12589 & -3.68366 \\
\hline & 6.08706 & -0.68466 & -4.40767 \\
\hline & 5.72384 & 0.58302 & -5.15684 \\
\hline & -5.20732 & -5.55983 & 2.71942 \\
\hline & -0.93579 & -6.01557 & 0.5363 \\
\hline
\end{tabular}




$\begin{array}{lrrr}\text { h } & 2.22994 & -3.89751 & -0.87299 \\ \text { h } & 5.98654 & -2.81203 & -3.11140 \\ \text { h } & 6.91616 & -0.50198 & -3.68428 \\ \text { h } & 6.40913 & -1.49060 & -5.10857 \\ \text { h } & 6.60567 & 0.95630 & -5.72034 \\ \text { h } & 4.89830 & 0.39235 & -5.87489 \\ \text { h } & 5.39357 & 1.37417 & -4.45089 \\ \text { h } & -3.17233 & -6.34402 & 1.70709 \\ \text { h } & -3.73679 & -2.06307 & 1.80757 \\ \text { h } & -1.52451 & -1.71616 & 0.63997\end{array}$

\section{OH-EEZE}

$E(B 3 L Y P /$ def2-TZVP $)=-1025.108962164$

32

$\begin{array}{llll}\text { C } & 1.17337 & -0.40063 & 3.81617\end{array}$

$\begin{array}{llll}\text { C } & -0.01600 & 0.02567 & 4.42512\end{array}$

C $\quad-1.11828 \quad 0.44965 \quad 3.66882$

$\begin{array}{llll}\text { C } & -1.03789 & 0.45294 & 2.27224\end{array}$

$\begin{array}{llll}\text { C } & 0.15272 & 0.02401 & 1.64933\end{array}$

$\begin{array}{llll}\text { C } & 1.25221 & -0.40528 & 2.42075\end{array}$

$\begin{array}{llll}\mathrm{n} & -0.10746 & 0.03148 & 5.90301\end{array}$

$\begin{array}{lllll}0 & -1.16830 & 0.40510 & 6.40480\end{array}$

$\begin{array}{lllll}0 & 0.33235 & 0.05184 & 0.28395\end{array}$

$\begin{array}{llll}0 & 0.88438 & -0.33632 & 6.53424\end{array}$

C $\quad-0.73506-0.34269-0.66152$

$\mathrm{n} \quad-0.36134 \quad-0.20039-1.95293$

c $\quad 0.818850 .18365-2.40818$

$\begin{array}{llll}\text { C } & 1.05271 & 0.28990 & -3.83490\end{array}$

$\begin{array}{llll}\text { c } & 0.06458 & -0.00577 & -4.72812\end{array}$

$\begin{array}{lllll}0 & 0.24231 & 0.08538 & -6.04460\end{array}$

$\begin{array}{llll}\text { c } & -0.88490 & -0.25287 & -6.88101\end{array}$

$\begin{array}{llll}\text { c } & -0.46869 & -0.08821 & -8.32955\end{array}$

$\begin{array}{lllll}0 & -1.78929 & -0.73881 & -0.22718\end{array}$

$\begin{array}{lllll}0 & 1.88707 & 0.50202 & -1.66906\end{array}$

h $\quad 2.16690 \quad-0.74402 \quad 1.90933$

h $\quad 2.04704 \quad 0.61758-4.17288$

h $\quad-0.92684-0.33429-4.35761$

h $\quad-1.19243-1.30191-6.66355$

h $\quad-1.736650 .41878-6.62492$

h $\quad-1.32009-0.34036-8.99730$

$\begin{array}{lllll}\text { h } & -0.15955 & 0.95877 & -8.53603\end{array}$

h $\quad 0.38122 \quad-0.75985 \quad-8.57624$

h $\quad 2.01276 \quad-0.72667 \quad 4.44656$

h $\quad-2.02835 \quad 0.77833 \quad 4.19078$

h $\quad-1.89225 \quad 0.77995 \quad 1.66616$

$\begin{array}{lllll}\text { h } & 1.60394 & 0.41256 & -0.70703\end{array}$

3OH-EZZE

$E(B 3 L Y P /$ def2-TZVP $)=-1025.107598489$

http://mc.manuscriptcentral.com/poc 
$\begin{array}{llll}\text { C } & 0.39457 & 0.07636 & 4.42081\end{array}$

C $\quad-0.626650 .89009 \quad 3.90922$

$\begin{array}{llll}\text { C } & -0.88025 & 0.89671 & 2.53348\end{array}$

$\begin{array}{llll}\text { C } & -0.10421 & 0.07956 & 1.68570\end{array}$

$\begin{array}{llll}\text { c } & 0.91577 & -0.73858 & 2.21304\end{array}$

$\begin{array}{llll}\text { C } & 1.17363 & -0.73841 & 3.58658\end{array}$

$\begin{array}{lllll}0 & -0.25439 & 0.07211 & 0.31643\end{array}$

$\begin{array}{llll}\text { n } & 0.66078 & 0.07855 & 5.87753\end{array}$

$\begin{array}{lllll}0 & 1.57397 & -0.63913 & 6.28814\end{array}$

$\begin{array}{lllll}0 & -0.04681 & 0.79827 & 6.58300\end{array}$

$\begin{array}{lllll}\text { C } & -1.58736 & 0.08701 & -0.33342\end{array}$

$\begin{array}{lllll}0 & -2.57503 & 0.08412 & 0.35811\end{array}$

$\begin{array}{lllll}\mathrm{n} & -1.50918 & 0.09942 & -1.68561\end{array}$

c $\quad-0.41206 \quad 0.05025-2.41700$

$\begin{array}{lllll}\text { o } & 0.84611 & -0.02988 & -1.95589\end{array}$

$\begin{array}{lllll}\text { C } & -0.54890 & 0.07969 & -3.85868\end{array}$

c $\quad 0.53056 \quad 0.04357-4.69219$

$\begin{array}{lllll}0 & 0.40199 & 0.07523 & -6.01971\end{array}$

c $\quad \begin{array}{llll}1.61466 & 0.01402 & -6.79762\end{array}$

$\begin{array}{lllll}\text { C } & 1.24454 & 0.11204 & -8.26491\end{array}$

$\begin{array}{llll}\text { h } & 1.49969 & -1.37541 & 1.53007\end{array}$

h $\quad-1.57182 \quad 0.13496 \quad-4.25762$

h $\quad \begin{array}{llll}1.55921 & -0.01216 & -4.28492\end{array}$

h $\quad 2.28367 \quad 0.85200 \quad-6.49220$

h $\quad 2.13737-0.94566-6.57576$

h $\quad 2.16204 \quad 0.06360-8.88933$

h $\quad 0.57425 \quad-0.72332-8.55977$

h $\quad 0.72437 \quad 1.07025 \quad-8.47841$

h $\quad 1.96256-1.36232 \quad 4.03002$

h $\quad-1.20890 \quad 1.51470 \quad 4.60175$

h $\quad-1.67707 \quad 1.52662 \quad 2.11746$

h $\quad 0.78067-0.02088-0.95085$

Transition state for the direct $2 \mathrm{NH}$ to Phenol Hydrogen Transfer $E(B 3 L Y P / d e f 2-T Z V P)=-1025.054946518$

32

$\begin{array}{lrrr}\text { c } & -1.20074 & -2.90995 & 1.02487 \\ \mathrm{c} & -1.01798 & -3.90695 & 0.02718 \\ \mathrm{c} & -1.67684 & -5.15845 & 0.17632 \\ \mathrm{c} & -2.49414 & -5.40124 & 1.27965 \\ \mathrm{c} & -2.65599 & -4.40011 & 2.25354 \\ \mathrm{c} & -2.01120 & -3.15761 & 2.13349 \\ \mathrm{o} & -0.22900 & -3.68880 & -1.02528 \\ \mathrm{c} & -0.83004 & -3.60704 & -3.03212 \\ \mathrm{o} & -1.32076 & -4.55204 & -3.52282 \\ \mathrm{n} & -3.51305 & -4.65873 & 3.42074 \\ \mathrm{o} & -3.63169 & -3.75705 & 4.25555 \\ \mathrm{o} & -4.06284 & -5.76149 & 3.49552 \\ \mathrm{n} & -0.35431 & -2.41818 & -2.88930 \\ \mathrm{c} & -0.18505 & -1.35809 & -3.87846\end{array}$


$\begin{array}{llll}0 & 0.32447 & -0.31927 & -3.49978\end{array}$

c $\quad-0.65262-1.67210-5.22533$

$\begin{array}{llll}\text { c } & -0.50816 & -0.72817 & -6.20072\end{array}$

$\begin{array}{llll}0 & -0.91362 & -0.92114 & -7.45050\end{array}$

$\begin{array}{llll}\text { C } & -0.63973 & 0.12685 & -8.40928\end{array}$

$\begin{array}{llll}\text { C } & -1.40703 & -0.17701 & -9.68046\end{array}$

h $\quad-1.11070-2.64519-5.46228$

h $\quad-1.52567 \quad-5.92974-0.59513$

h $\quad-0.08190-2.55889-1.71153$

h $\quad-0.04008 \quad 0.24679 \quad-5.95503$

h $\quad-0.94494 \quad 1.10490-7.97167$

h $\quad 0.45861 \quad 0.15474 \quad-8.59475$

h $\quad-1.19859 \quad 0.60607-10.44044$

h $\quad-1.10423-1.16004-10.09988$

h $\quad-2.50116-0.19826-9.48926$

$\begin{array}{lllll}\text { h } & -3.01411 & -6.36087 & 1.41394\end{array}$

$\begin{array}{llll}\text { h } & -2.16317 & -2.40345 & 2.91906\end{array}$

h $\quad-0.68145 \quad-1.94407 \quad 0.91472$

Transition State for EEZE isomer $E(B 3 L Y P /$ def2-TZVP $)=-1025.096002111$

32

$\begin{array}{llll}\text { C } & 0.16291 & -0.20954 & 4.27651\end{array}$

$\begin{array}{llll}\text { C } & -1.07807 & 0.26637 & 3.82403\end{array}$

$\begin{array}{llll}\text { C } & -1.20089 & 0.72057 & 2.50857\end{array}$

$\begin{array}{llll}\text { C } & -0.08210 & 0.69061 & 1.64079\end{array}$

$\begin{array}{llll}\text { C } & 1.16036 & 0.19997 & 2.11560\end{array}$

$\begin{array}{llll}\text { C } & 1.28604 & -0.24234 & 3.43412\end{array}$

$\begin{array}{lllll}0 & -0.19555 & 1.14336 & 0.37149\end{array}$

$\begin{array}{llll}\text { n } & 0.29262 & -0.68279 & 5.66852\end{array}$

o $\quad 1.39933-1.08757 \quad 6.03260$

$\begin{array}{llll}0 & -0.71315 & -0.64525 & 6.38075\end{array}$

C $\quad-1.333880 .26202-0.78799$

$\begin{array}{lllll}0 & -2.32733 & -0.05432 & -0.22439\end{array}$

$\begin{array}{lllll}\mathrm{n} & -0.75638 & 0.23287 & -1.94735\end{array}$

c $\quad 0.51050 \quad 0.59086-2.27214$

$\begin{array}{lllll}0 & 1.36203 & 1.09210 & -1.43644\end{array}$

$\begin{array}{lllll}\text { c } & 0.94634 & 0.40656 & -3.63810\end{array}$

$\begin{array}{llll}\text { c } & 0.11799 & -0.12558 & -4.58597\end{array}$

$\begin{array}{llll}\text { o } & 0.50493 & -0.30502 & -5.84381\end{array}$

$\begin{array}{llll}\text { C } & -0.46876 & -0.85276 & -6.76345\end{array}$

$\begin{array}{llll}\text { C } & 0.21396 & -1.07980 & -8.09747\end{array}$

$\begin{array}{llll}\text { h } & 2.02512 & 0.17286 & 1.43307\end{array}$

$\begin{array}{lllll}\text { h } & 1.97387 & 0.71030 & -3.88688\end{array}$

h $\quad-0.91414 \quad-0.42500-4.31512$

h $\quad-0.86546 \quad-1.80457-6.34177$

h $\quad-1.31361-0.13240-6.85673$

h $\quad-0.51491-1.49447-8.82612$

h $\quad 0.61295-0.12680-8.50541$

h $\quad 1.05537-1.79798-7.99550$

h $\quad 2.23870-0.62230 \quad 3.82999$

$\begin{array}{llll}\text { h } & -1.92907 & 0.27889 & 4.52017\end{array}$ 
h $\quad-2.15743 \quad 1.10926 \quad 2.13230$

h $\quad 0.80125 \quad 1.18219 \quad-0.44498$

6

Transition State for EZZE isomer $E(B 3 L Y P /$ def2-TZVP $)=-1025.095291990$

32

$\begin{array}{llll}\text { C } & -0.63501 & 0.55911 & 3.94289\end{array}$

C $\quad-0.97909 \quad 1.00395 \quad 2.66409$

C $\quad-0.16967 \quad 0.66336 \quad 1.55236$

$\begin{array}{llll}\text { C } & 0.99097 & -0.12780 & 1.74923\end{array}$

c $\quad 1.33959-0.564853 .02840$

$\begin{array}{lllll}\text { C } & 0.52108 & -0.21877 & 4.11581\end{array}$

$\begin{array}{lllll}0 & -0.48513 & 1.10769 & 0.31651\end{array}$

h $\quad \begin{array}{llll}\text { h } & 1.61338 & -0.39574 & 0.88017\end{array}$

$\begin{array}{llll}\text { c } & -2.09298 & 0.59036 & -0.45292\end{array}$

$\begin{array}{lllll}\mathrm{n} & -1.87287 & 0.39583 & -1.71688\end{array}$

$\begin{array}{llll}\text { c } & -0.69296 & 0.36987 & -2.37330\end{array}$

$\begin{array}{llll}\text { c } & -0.74891 & 0.07154 & -3.78690\end{array}$

h $\quad-1.73428 \quad-0.12400-4.23359$

$\begin{array}{llll}0 & -2.95189 & 0.57645 & 0.36211\end{array}$

$\begin{array}{lllll}0 & 0.46001 & 0.59168 & -1.81533\end{array}$

c $\quad 0.39031 \quad 0.03997-4.54120$

o $\quad 0.37174-0.22355-5.84321$

$\begin{array}{llll}\text { C } & 1.64456 & -0.27005 & -6.52865\end{array}$

c $\quad 1.38167-0.44195-8.01120$

$\begin{array}{lllll}\text { h } & 1.37486 & 0.24085 & -4.07381\end{array}$

h $\quad 2.20041 \quad 0.67279-6.31896$

h $\quad 2.23564-1.12159-6.11916$

h $\quad 2.34635-0.49363-8.55946$

h $\quad 0.81672-1.37831-8.20688$

$\begin{array}{llll}\text { h } & 0.79550 & 0.41217 & -8.41239\end{array}$

h $\quad 2.23525-1.17636 \quad 3.20883$

n $\quad 0.88776-0.68171 \quad 5.46793$

h $\quad-1.24456 \quad 0.80962 \quad 4.82310$

$\begin{array}{llll}\text { h } & -1.87196 & 1.62453 & 2.50478\end{array}$

$\begin{array}{lllll}\text { h } & 0.23373 & 0.84141 & -0.73378\end{array}$

$\begin{array}{llll}\text { o } & 0.15199 & -0.35687 & 6.40250\end{array}$

o $\quad 1.90903-1.364595 .57969$

Product Complex EEZE

$E(B 3 L Y P /$ def2-TZVP) $=-1025.123266873$

32

C $\quad 1.21722 \quad-0.45952 \quad 2.95524$

$\begin{array}{llll}\text { c } & 0.41616 & -0.41451 & 4.10800\end{array}$

$\begin{array}{llll}\text { c } & -0.74290 & 0.38081 & 4.15622\end{array}$

$\begin{array}{llll}\text { C } & -1.10346 & 1.13428 & 3.04022\end{array}$

c $\quad-0.31136 \quad 1.10053 \quad 1.86503$

$\begin{array}{llll}\text { C } & 0.85652 & 0.29474 & 1.83691\end{array}$ 
n $\quad 0.79570 \quad-1.21266 \quad 5.28320$

$\begin{array}{llll}0 & 0.06799 & -1.14970 & 6.27843\end{array}$

$\begin{array}{lllll}0 & -0.70519 & 1.84765 & 0.81803\end{array}$

$\begin{array}{llll}0 & 1.81890 & -1.89958 & 5.20459\end{array}$

C $\quad-2.250320 .56384-1.43155$

$\begin{array}{lllll}\mathrm{n} & -1.33496 & 0.46372 & -2.24536\end{array}$

$\begin{array}{llll}\text { c } & 0.04198 & 0.76541 & -2.32563\end{array}$

$\begin{array}{llll}\text { c } & 0.68077 & 0.39330 & -3.57417\end{array}$

C $\quad 0.00738-0.20501-4.60100$

o $\quad 0.60308-0.53659-5.74161$

$\begin{array}{llll}\text { C } & -0.20919 & -1.18058 & -6.75019\end{array}$

C $\quad 0.64758-1.40473-7.98002$

$\begin{array}{lllll}0 & -3.22287 & 0.58543 & -0.77550\end{array}$

$\begin{array}{lllll}0 & 0.65868 & 1.30924 & -1.39865\end{array}$

$\begin{array}{llll}\text { h } & 1.47683 & 0.27367 & 0.92641\end{array}$

$\begin{array}{lllll}\text { h } & 1.75398 & 0.61956 & -3.66082\end{array}$

h $\quad-1.07402-0.43165-4.50852$

h $\quad-0.58976-2.14372-6.33879$

h $\quad-1.08308 \quad-0.52719-6.97702$

h $\quad 0.04667 \quad-1.90367 \quad-8.77004$

h $\quad 1.02402-0.44015-8.38238$

h $\quad 1.51921-2.05123-7.74296$

h $\quad 2.11765-1.09056 \quad 2.95840$

h $\quad-1.34159 \quad 0.38955 \quad 5.07820$

$\begin{array}{lllll}\text { h } & -2.00541 & 1.76561 & 3.04458\end{array}$

$\begin{array}{llll}\text { h } & -0.09417 & 1.70866 & 0.02373\end{array}$

Product Complex EEZE

$E(B 3 L Y P / d e f 2-T Z V P)=-1025.123503031$

32

$\begin{array}{llll}\text { C } & -0.18613 & 0.49057 & 4.01275\end{array}$

c $\quad \begin{array}{llll}-0.80079 & 1.20329 & 2.98440\end{array}$

$\begin{array}{llll}\text { C } & -0.36077 & 1.05484 & 1.64489\end{array}$

C $\quad \begin{array}{llll}\text { c } & 0.71377 & 0.17158 & 1.36132\end{array}$

$\begin{array}{llll}\text { c } & 1.32901 & -0.54188 & 2.39169\end{array}$

$\begin{array}{llll}\text { C } & 0.87574 & -0.38128 & 3.71132\end{array}$

$\begin{array}{lllll}0 & -0.98364 & 1.77086 & 0.69268\end{array}$

$\begin{array}{llll}\text { h } & 1.05900 & 0.05832 & 0.32102\end{array}$

c $\quad-3.209110 .68418-1.04378$

$\begin{array}{lllll}\mathrm{n} & -2.51769 & 0.41919 & -2.02333\end{array}$

c $\quad-1.18920 \quad 0.52581 \quad-2.45874$

$\begin{array}{llll}\text { c } & -0.98078 & -0.00116 & -3.79715\end{array}$

h $\quad-1.83363-0.42525-4.34760$

$\begin{array}{lllll}\text { o } & -4.00204 & 0.86909 & -0.19892\end{array}$

$\begin{array}{lllll}0 & -0.29255 & 1.02530 & -1.75651\end{array}$

$\begin{array}{lllll}\text { C } & 0.26787 & 0.03807 & -4.35018\end{array}$

$\begin{array}{lllll}0 & 0.52269 & -0.42060 & -5.56937\end{array}$

$\begin{array}{llll}\text { C } & 1.89318 & -0.36025 & -6.02898\end{array}$

$\begin{array}{llll}\text { C } & 1.92604 & -0.78464 & -7.48355\end{array}$

h $\quad \begin{array}{llll}\text { h } & 1.11227 & 0.46864 & -3.77452\end{array}$

h $\quad 2.27030 \quad 0.68011 \quad-5.89863$

h $\quad 2.50860-1.03758-5.39366$

http://mc.manuscriptcentral.com/poc 
h $\quad 2.97173 \quad-0.75972 \quad-7.85776$

h $\quad 1.53648$ - $1.81798-7.60318$

h $\quad 1.31279-0.10240-8.11005$

h $\quad 2.16433 \quad-1.23027 \quad 2.19782$

n $\quad 1.52420 \quad-1.13513 \quad 4.79425$

h $\quad-0.51166 \quad 0.58873 \quad 5.05838$

h $\quad-1.63494 \quad 1.89204 \quad 3.18826$

$\begin{array}{llll}\text { h } & -0.61846 & 1.54857 & -0.22503\end{array}$

$\begin{array}{llll}\text { o } & 1.09652 & -0.97486 & 5.94095\end{array}$

$\begin{array}{llll}0 & 2.45807 & -1.88478 & 4.49199\end{array}$

Molecular energies (in a.u.) and molecular structures of $7 d$ (Table S4)

\section{NH-EEEE}

$E(B 3 L Y P /$ def2-TZVP $)=-1064.420316614$

35

$\begin{array}{llll}\text { C } & -0.98248 & -3.74696 & 0.89429\end{array}$

$\begin{array}{llll}\text { C } & -0.98226 & -5.14278 & 0.86467\end{array}$

$\begin{array}{llll}\text { C } & -1.64964 & -5.84098 & 1.88234\end{array}$

c $\quad-2.30769-5.17068 \quad 2.92182$

$\begin{array}{llll}\text { c } & -2.31108 & -3.77031 & 2.95737\end{array}$

C $\quad-1.64558-3.06201 \quad 1.93542$

$\begin{array}{lllll}\text { o } & -1.52914 & -1.68923 & 1.86224\end{array}$

$\begin{array}{llll}\text { C } & -2.37170 & -0.80510 & 2.49600\end{array}$

$\begin{array}{llll}\text { o } & -3.28831 & -1.10318 & 3.23415\end{array}$

h $\quad-0.46909-3.159210 .11710$

$\begin{array}{llll}\text { n } & -2.02728 & 0.50526 & 2.19181\end{array}$

c $\quad-0.85848 \quad 1.08253 \quad 1.56764$

$\begin{array}{llll}0 & -0.32977 & 2.03047 & 2.13571\end{array}$

$\begin{array}{llll}\text { C } & -0.41301 & 0.56993 & 0.26079\end{array}$

$\begin{array}{llll}\text { C } & -1.30499 & -0.05622 & -0.56066\end{array}$

o $\quad-0.96021-0.52485-1.77151$

$\begin{array}{llll}\text { C } & -2.03262 & -0.95674 & -2.62863\end{array}$

$\begin{array}{llll}\text { C } & -1.43136 & -1.59417 & -3.86695\end{array}$

C $\quad 0.99835 \quad 0.88753-0.15425$

$\begin{array}{llll}\text { h } & -2.45093 & 1.15955 & 2.86082\end{array}$

$\begin{array}{lllll}\text { h } & & 1.14989 & 0.69488 & -1.23531\end{array}$

$\begin{array}{llll}\text { h } & 1.73264 & 0.27274 & 0.41534\end{array}$

$\begin{array}{lllll}\text { h } & 1.22928 & 1.95077 & 0.07038\end{array}$

h $\quad-2.36174 \quad-0.20115-0.26706$

h $\quad-2.67517 \quad-1.68022 \quad-2.07271$

h $\quad-2.66053-0.07463-2.89613$

h $\quad-2.24027 \quad-1.92923-4.55080$

h $\quad-0.79011-0.86905-4.41223$

h $\quad-0.81115-2.47608-3.59811$

$\begin{array}{llll}\text { h } & -0.47570 & -5.70697 & 0.06880\end{array}$

$\begin{array}{llll}\text { n } & -1.65540 & -7.32210 & 1.85662\end{array}$

h $\quad-2.81316 \quad-5.76023 \quad 3.70019$

h $\quad-2.82778$-3.22974 3.75895 
$\begin{array}{llll}0 & -2.24527 & -7.90325 & 2.76749 \\ 0 & -1.06951 & -7.87412 & 0.92422\end{array}$

2NH-EEEZ

$E(B 3 L Y P /$ def2-TZVP $)=-1064.425317291$

35

$\begin{array}{llll}\text { C } & -2.11683 & -4.93177 & 0.33547\end{array}$

$\begin{array}{llll}\text { C } & -2.07617 & -5.66853 & 1.52746\end{array}$

C $\quad-1.82523 \quad-5.054562 .76346$

C $\quad-1.61835 \quad-3.67325 \quad 2.79992$

c $\quad-1.65996-2.92243 \quad 1.60785$

$\begin{array}{llll}\text { C } & -1.90719 & -3.54821 & 0.36884\end{array}$

$\begin{array}{lllll}0 & -1.37796 & -1.57937 & 1.74739\end{array}$

$\begin{array}{llll}\text { C } & -1.97860 & -0.61102 & 0.94138\end{array}$

$\begin{array}{llll}\mathrm{n} & -1.43527 & 0.60193 & 1.31734\end{array}$

c $\quad-1.36605 \quad 1.86048 \quad 0.60918$

C $\quad-2.50682 \quad 2.36283-0.16338$

c $\quad-2.218413 .51358-1.09310$

h $\quad-1.93937-2.95902-0.55624$

$\begin{array}{llll}0 & -2.82480 & -0.84128 & 0.10513\end{array}$

$\begin{array}{lllll}0 & -0.31519 & 2.48322 & 0.72770\end{array}$

$\begin{array}{llll}\text { C } & -3.77283 & 1.89671 & 0.05539\end{array}$

$\begin{array}{lllll}0 & -4.83636 & 2.40340 & -0.58278\end{array}$

$\begin{array}{llll}\text { c } & -6.10950 & 1.79414 & -0.29835\end{array}$

$\begin{array}{llll}\text { c } & -7.17119 & 2.49928 & -1.12084\end{array}$

$\begin{array}{llll}\text { h } & -0.63619 & 0.52105 & 1.95780\end{array}$

h $\quad-3.12838 \quad 3.80568-1.65393$

h $\quad-1.41815 \quad 3.24807-1.81823$

$\begin{array}{lllll}\text { h } & -1.84674 & 4.39538 & -0.52538\end{array}$

$\begin{array}{llll}\text { h } & -3.98329 & 1.08861 & 0.77512\end{array}$

h $\quad-6.05526 \quad 0.70981-0.55043$

h $\quad-6.32039 \quad 1.88502 \quad 0.79333$

h $\quad-8.16662 \quad 2.04820-0.92061$

h $\quad-7.21742 \quad 3.57954-0.86509$

h $\quad-6.95604 \quad 2.40564 \quad-2.20684$

h $\quad-2.30942-5.45788-0.61053$

$\begin{array}{llll}\text { n } & -2.29831 & -7.13259 & 1.48104\end{array}$

h $\quad-1.79911 \quad-5.66957 \quad 3.67424$

h $\quad-1.41792 \quad-3.15234 \quad 3.74866$

$\begin{array}{lllll}\text { o } & -2.50967 & -7.63948 & 0.37894\end{array}$

$\begin{array}{llll}0 & -2.25664 & -7.74590 & 2.54818\end{array}$

2NH-EEZE

$E(B 3 L Y P /$ def2-TZVP $)=-1064.423425480$

35

$\begin{array}{llll}\text { C } & -3.13200 & -4.54511 & 0.49779\end{array}$

$\begin{array}{llll}\text { C } & -2.38220 & -5.34523 & 1.37001\end{array}$

$\begin{array}{llll}\text { C } & -1.26240 & -4.84202 & 2.05091\end{array}$

http://mc.manuscriptcentral.com/poc 
$\begin{array}{llll}\text { C } & -0.89399 & -3.51151 & 1.84745\end{array}$

C $\quad-1.64315-2.69873 \quad 0.97060$

$\begin{array}{llll}\text { C } & -2.76788 & -3.20831 & 0.28943\end{array}$

$\begin{array}{lllll}0 & -1.13731 & -1.42184 & 0.84656\end{array}$

$\begin{array}{llll}\text { c } & -1.81187 & -0.36411 & 0.31868\end{array}$

$\begin{array}{lllll}\mathrm{n} & -1.04666 & 0.79595 & 0.40669\end{array}$

$\begin{array}{llll}\text { c } & 0.13098 & 1.08106 & 1.16469\end{array}$

C $\quad 0.78181 \quad 2.37873 \quad 0.83749$

$\begin{array}{llll}\text { C } & 1.70845 & 2.94076 & 1.88179\end{array}$

h $\quad-3.34567 \quad-2.56427 \quad-0.38373$

$\begin{array}{lllll}0 & -2.91386 & -0.38415 & -0.19484\end{array}$

$\begin{array}{lllll}0 & 0.55873 & 0.32880 & 2.02670\end{array}$

c $\quad 0.62213 \quad 2.96246-0.38616$

$\begin{array}{lllll}\text { o } & 1.21928 & 4.12261 & -0.71203\end{array}$

C $\quad 1.15560 \quad 4.52227-2.09058$

C $\quad 1.72550 \quad 5.92282-2.21164$

$\begin{array}{llll}\text { h } & -1.52212 & 1.58992 & -0.02975\end{array}$

h $\quad 2.36059 \quad 3.72974 \quad 1.45646$

h $\quad 2.33272 \quad 2.125192 .30512$

h $\quad 1.13951 \quad 3.38102 \quad 2.73265$

$\begin{array}{lllll}\text { h } & 0.01748 & 2.49640 & -1.18967\end{array}$

h $\quad 1.73774 \quad 3.79786-2.70803$

h $\quad 0.09386 \quad 4.48932-2.43408$

h $\quad \begin{array}{llll}1.69301 & 6.25522 & -3.27128\end{array}$

h $\quad 1.14096 \quad 6.64198-1.59880$

h $\quad 2.78192 \quad 5.94945-1.86890$

h $\quad-4.00025 \quad-4.98473-0.01367$

$\begin{array}{llll}\text { n } & -2.77904 & -6.75678 & 1.57726\end{array}$

h $\quad-0.70243 \quad-5.50211 \quad 2.72843$

h $\quad-0.02711 \quad-3.063862 .35692$

$\begin{array}{lllll}0 & -3.76356 & -7.16643 & 0.96070\end{array}$

$\begin{array}{llll}0 & -2.09793 & -7.42935 & 2.35243\end{array}$

\section{NH-EEZZ}

$E(B 3 L Y P /$ def2-TZVP $)=-1064.422157366$

35

$\begin{array}{llll}\text { C } & -2.96713 & -3.13315 & 0.74547\end{array}$

c $\quad-1.58740-2.838810 .74092$

$\begin{array}{llll}\text { c } & -0.63648 & -3.84297 & 1.01792\end{array}$

C $\quad-1.05394 \quad-5.14540 \quad 1.30013$

C $\quad-2.42755 \quad-5.43046 \quad 1.29832$

$\begin{array}{llll}\text { c } & -3.38183 & -4.44054 & 1.02658\end{array}$

$\begin{array}{lllll}0 & -1.06116 & -1.58188 & 0.53467\end{array}$

C $\quad-1.69952-0.61978-0.26121$

$\begin{array}{llll}\text { o } & -2.72207 & -0.78372 & -0.87483\end{array}$

h $\quad 0.43196 \quad-3.57823 \quad 1.00901$

$\begin{array}{lllll}\mathrm{n} & -0.89950 & 0.51029 & -0.20016\end{array}$

c $\quad-1.233881 .79922-0.71500$

$\begin{array}{lllll}0 & -2.35845 & 2.06573 & -1.10396\end{array}$

$\begin{array}{llll}\text { C } & -0.11906 & 2.78547 & -0.71737\end{array}$

$\begin{array}{llll}\text { C } & 1.18560 & 2.38469 & -0.73291\end{array}$

$\begin{array}{llll}\text { o } & 2.20622 & 3.26123 & -0.74729\end{array}$ 


$\begin{array}{lrrr}\text { c } & 3.51768 & 2.73203 & -0.99760 \\ \text { c } & 4.53279 & 3.83595 & -0.76779 \\ \text { c } & -0.51671 & 4.23401 & -0.80945 \\ \text { h } & -0.05852 & 0.42415 & 0.37594 \\ \text { h } & 0.34309 & 4.86781 & -1.10541 \\ \text { h } & -0.90636 & 4.60882 & 0.16475 \\ \text { h } & -1.34012 & 4.34815 & -1.54635 \\ \text { h } & 1.47723 & 1.31586 & -0.76611 \\ \text { h } & 3.70218 & 1.86794 & -0.31477 \\ \text { h } & 3.56528 & 2.35521 & -2.04670 \\ \text { h } & 5.55825 & 3.45515 & -0.96234 \\ \text { h } & 4.34304 & 4.69319 & -1.44854 \\ \text { h } & 4.48646 & 4.20419 & 0.27945 \\ \text { h } & -0.33824 & -5.95032 & 1.51962 \\ \text { h } & -2.87786 & -6.80980 & 1.59414 \\ \text { h } & -4.44789 & -4.70892 & 1.04077 \\ \text { h } & -3.70411 & -2.35198 & 0.52283 \\ \text { o } & -4.09033 & -7.02398 & 1.59275 \\ \text { o } & -2.00873 & -7.65252 & 1.82266\end{array}$

2NH-EZEE

$E(B 3 L Y P /$ def2-TZVP $)=-1064.421188748$

35

C $\quad-1.30544 \quad-3.44695 \quad 1.80554$

c $\quad-2.23428-4.433512 .15608$

$\begin{array}{llll}\text { C } & -3.59777 & -4.22309 & 1.90701\end{array}$

C $\quad-4.06230 \quad-3.04191 \quad 1.30979$

C $\quad-3.13765-2.05550 \quad 0.95800$

$\begin{array}{llll}\text { c } & -1.76470 & -2.25745 & 1.20377\end{array}$

$\begin{array}{llll}\text { o } & -0.93878 & -1.24968 & 0.75255\end{array}$

c $\quad 0.27577 \quad-0.97164 \quad 1.34833$

$\begin{array}{llll}\mathrm{o} & 0.65082 & -1.41646 & 2.41204\end{array}$

$\begin{array}{llll}\text { h } & -0.23670 & -3.59432 & 2.00597\end{array}$

$\begin{array}{lllll}\mathrm{n} & 0.98588 & -0.06110 & 0.58534\end{array}$

c $\quad 0.64818 \quad 0.65780-0.61259$

$\begin{array}{lllll}\text { o } & 0.94048 & 1.84994 & -0.64610\end{array}$

$\begin{array}{llll}\text { C } & 0.07310 & -0.09843 & -1.74532\end{array}$

c $\quad-0.466820 .68452-2.72785$

$\begin{array}{lllll}0 & -0.97674 & 0.15565 & -3.84760\end{array}$

C $\quad-1.495391 .07469-4.82679$

$\begin{array}{llll}\text { C } & -2.03784 & 0.27151 & -5.99372\end{array}$

c $\quad 0.15821-1.59597 \quad-1.91300$

$\begin{array}{llll}\text { h } & 1.70199 & 0.42592 & 1.13608\end{array}$

h $\quad-0.81413-2.10304-1.72580$

h $\quad 0.91212-2.04170-1.23205$

h $\quad 0.45312-1.84064-2.95694$

$\begin{array}{llll}\text { h } & -0.48790 & 1.78589 & -2.62263\end{array}$

h $\quad-2.29473 \quad 1.69399-4.35640$

h $\quad-0.67713 \quad 1.75852-5.15245$

h $\quad-2.44406 \quad 0.95608$-6.76869

$\begin{array}{llll}\text { h } & -1.23652 & -0.34425 & -6.45548\end{array}$

http://mc.manuscriptcentral.com/poc 
h $\quad-2.85274 \quad-0.40716-5.66247$

h $\quad-1.91642 \quad-5.37617 \quad 2.62410$

$\begin{array}{llll}\mathrm{n} & -4.57238 & -5.27450 & 2.27919\end{array}$

h $\quad-5.14008-2.91698 \quad 1.13372$

h $\quad-3.45846-1.11383 \quad 0.48703$

$\begin{array}{llll}0 & -5.76197 & -5.05001 & 2.05296\end{array}$

$\begin{array}{llll}0 & -4.12896 & -6.30461 & 2.78807\end{array}$

10

\section{NH-EZEZ}

$E(B 3 L Y P /$ def2-TZVP $)=-1064.424673427$

35

$\begin{array}{llll}\text { C } & -1.63780 & -3.22889 & 0.03439\end{array}$

C $\quad-1.48628-4.61117-0.12599$

$\begin{array}{llll}\text { C } & -1.76787 & -5.46940 & 0.94603\end{array}$

C $\quad-2.19738-4.97843 \quad 2.18782$

$\begin{array}{llll}\text { C } & -2.35126 & -3.59960 & 2.35016\end{array}$

C $\quad-2.07449-2.72743 \quad 1.27796$

$\begin{array}{llll}0 & -2.20475 & -1.38524 & 1.56804\end{array}$

C $\quad-2.59280-0.45343 \quad 0.60480$

$\begin{array}{lllll}0 & -2.90978 & -0.71982 & -0.53199\end{array}$

h $\quad-1.42725 \quad-2.54716-0.79892$

$\begin{array}{llll}\mathrm{n} & -2.53825 & 0.78733 & 1.20848\end{array}$

$\begin{array}{llll}\text { c } & -2.66680 & 2.08172 & 0.60245\end{array}$

$\begin{array}{llll}0 & -1.92122 & 2.96113 & 1.02766\end{array}$

C $\quad-3.71934 \quad 2.28133-0.41447$

C $\quad-3.56761 \quad 3.42391-1.15163$

$\begin{array}{lllll}0 & -4.45325 & 3.78814 & -2.08731\end{array}$

C $\quad-4.20011 \quad 5.02551-2.77753$

C $\quad-5.28601 \quad 5.22131-3.81862$

C $\quad-4.92570 \quad 1.39282-0.58953$

$\begin{array}{llll}\text { h } & -2.00344 & 0.82588 & 2.08375\end{array}$

h $\quad-5.79678 \quad 2.00472 \quad-0.90689$

h $\quad-5.18954 \quad 0.88021 \quad 0.36004$

h $\quad-4.75903 \quad 0.60367 \quad-1.35271$

h $\quad-2.69792 \quad 4.08726-0.98487$

h $\quad-4.19508 \quad 5.86022-2.03765$

h $\quad-3.19144 \quad 4.97862-3.25065$

h $\quad-5.11748 \quad 6.17191-4.36850$

h $\quad-5.28531 \quad 4.38638-4.55183$

h $\quad-6.28796 \quad 5.26541-3.34052$

h $\quad-1.14721-5.04193-1.07893$

$\begin{array}{llll}\text { n } & -1.60533 & -6.93050 & 0.76586\end{array}$

$\begin{array}{llll}\text { h } & -2.40637 & -5.68608 & 3.00251\end{array}$

h $\quad-2.68674-3.17238 \quad 3.30743$

$\begin{array}{llll}\text { o } & -1.86865 & -7.65263 & 1.72807\end{array}$

$\begin{array}{llll}0 & -1.21727 & -7.32703 & -0.33367\end{array}$

2NH-EZZE

$E(B 3 L Y P /$ def2-TZVP $)=-1064.426084283$ 
35

$\begin{array}{lrrr}\text { c } & -2.75755 & -3.67544 & 0.26139 \\ \mathrm{c} & -3.29674 & -4.80146 & 0.89812 \\ \mathrm{c} & -2.74621 & -5.25413 & 2.10451 \\ \mathrm{c} & -1.65441 & -4.60474 & 2.70276 \\ \mathrm{c} & -1.11108 & -3.48426 & 2.07355 \\ \mathrm{c} & -1.65980 & -3.02036 & 0.85812 \\ \mathrm{o} & -1.00604 & -1.91262 & 0.36269 \\ \mathrm{c} & -1.35483 & -1.22880 & -0.75951 \\ \mathrm{o} & -2.29267 & -1.46598 & -1.49724 \\ \mathrm{~h} & -3.17619 & -3.30382 & -0.68088 \\ \mathrm{n} & -0.48054 & -0.16969 & -0.98851 \\ \mathrm{c} & 0.66705 & 0.28396 & -0.27943 \\ \mathrm{o} & 1.07383 & -0.25781 & 0.74046 \\ \mathrm{c} & 1.30508 & 1.47213 & -0.91452 \\ \mathrm{c} & 2.42235 & 1.92453 & -0.27359 \\ \mathrm{o} & 3.12883 & 2.97905 & -0.70214 \\ \mathrm{c} & 4.28670 & 3.34094 & 0.07243 \\ \mathrm{c} & 4.95513 & 4.52866 & -0.59327 \\ \mathrm{c} & 0.78053 & 2.13542 & -2.16427 \\ \mathrm{~h} & -0.74604 & 0.34505 & -1.83248 \\ \mathrm{~h} & 1.41716 & 3.00347 & -2.43209 \\ \mathrm{~h} & -0.25870 & 2.51942 & -2.03599 \\ \mathrm{~h} & 0.78093 & 1.44739 & -3.04213 \\ \mathrm{~h} & 2.77212 & 1.40692 & 0.64026 \\ \mathrm{~h} & 3.96629 & 3.58848 & 1.11145 \\ \mathrm{~h} & 4.97737 & 2.46693 & 0.12478 \\ \mathrm{~h} & 5.85438 & 4.83089 & -0.01490 \\ \mathrm{~h} & 5.27241 & 4.27531 & -1.62746 \\ \mathrm{~h} & 4.26261 & 5.39611 & -0.64222 \\ \mathrm{~h} & -4.15124 & -5.34213 & 0.46650 \\ \mathrm{n} & -3.32751 & -6.44526 & 2.76396 \\ \mathrm{~h} & -1.25187 & -4.99150 & 3.64940 \\ \mathrm{~h} & -0.25353 & -2.93902 & 2.49613 \\ \mathrm{o} & -2.82035 & -6.80827 & 3.82668 \\ \mathrm{o} & -4.27882 & -6.99603 & 2.20850\end{array}$

\section{NH-EZZZ}

$E(B 3 L Y P /$ def2-TZVP $)=-1064.425376873$

35

$\begin{array}{lrrr}\text { c } & -3.56301 & -4.39865 & 1.42279 \\ \text { c } & -3.10881 & -3.11184 & 1.11038 \\ \text { c } & -1.75021 & -2.92699 & 0.77800 \\ \text { c } & -0.85815 & -4.01967 & 0.76386 \\ \text { c } & -1.31423 & -5.30160 & 1.07843 \\ \text { c } & -2.66779 & -5.47706 & 1.40260 \\ \text { o } & -1.17652 & -1.70459 & 0.50406 \\ \text { c } & -1.89809 & -0.64762 & -0.07329 \\ \text { n } & -1.00946 & 0.41192 & -0.15209 \\ \text { c } & -1.28660 & 1.70272 & -0.68265 \\ \text { c } & -0.11387 & 2.61965 & -0.63348\end{array}$

http://mc.manuscriptcentral.com/poc 
2NH-ZEEE

$E(B 3 L Y P /$ def2-TZVP $)=-1064.417018305$

35

$\begin{array}{llll}\text { C } & -0.80318 & -5.07483 & 0.86763\end{array}$

$\begin{array}{llll}\text { C } & -1.95099 & -5.72096 & 1.35202\end{array}$

c $\quad-2.91296-5.03545 \quad 2.10536$

C $\quad-2.74122 \quad-3.673892 .38736$

C $\quad-1.59587-3.01254 \quad 1.89170$

c $\quad-0.62955-3.71687 \quad 1.13904$

$\begin{array}{llll}0 & -1.29316 & -1.68296 & 2.06864\end{array}$

$\begin{array}{llll}\text { C } & -2.13205 & -0.75808 & 2.64634\end{array}$

$\begin{array}{lllll}\mathrm{n} & -1.66438 & 0.52217 & 2.40447\end{array}$

c $\quad-0.69459 \quad 1.05427 \quad 1.50850$

$\begin{array}{llll}\text { C } & -0.47533 & 0.43399 & 0.17757\end{array}$

c $\quad 0.842220 .78305 \quad-0.47225$

$\begin{array}{llll}\text { h } & 0.25572 & -3.17160 & 0.77759\end{array}$

$\begin{array}{lllll}\text { o } & -3.09851 & -1.00676 & 3.33744\end{array}$

$\begin{array}{llll}0 & -0.10918 & 2.07798 & 1.84656\end{array}$

$\begin{array}{llll}\text { c } & -1.43175 & -0.22796 & -0.53865\end{array}$

$\begin{array}{lllll}0 & -2.66997 & -0.51028 & -0.09430\end{array}$

C $\quad-3.51857-1.28442-0.96138$

c $\quad-4.91228-1.31230-0.36258$

h $\quad \begin{array}{llll}-1.99709 & 1.21190 & 3.08733\end{array}$

h $\quad 0.917890 .35464 \quad-1.49435$

$\begin{array}{llll}\text { h } & 1.69983 & 0.40320 & 0.12753\end{array}$

h $\quad 0.96769 \quad 1.88589-0.53705$

h $\quad-1.21399-0.54652-1.57836$ 


$\begin{array}{lrrr}\text { h } & -3.52337 & -0.82049 & -1.97584 \\ \text { h } & -3.09863 & -2.31387 & -1.05198 \\ \text { h } & -5.58552 & -1.92391 & -1.00058 \\ \text { h } & -4.89392 & -1.75592 & 0.65595 \\ \text { h } & -5.33203 & -0.28626 & -0.28856 \\ \text { h } & -0.06812 & -5.64917 & 0.28611 \\ \text { h } & -2.14663 & -7.15957 & 1.06178 \\ \text { h } & -3.79220 & -5.58453 & 2.47235 \\ \text { h } & -3.47218 & -3.12563 & 2.99321 \\ \text { o } & -1.27319 & -7.72837 & 0.40539 \\ \text { o } & -3.17006 & -7.69178 & 1.49287\end{array}$

2NH-ZEEZ

$E(B 3 L Y P /$ def2-TZVP $)=-1064.421034889$

35

C $\quad-2.14908 \quad-5.63854 \quad 1.41379$

$\begin{array}{llll}\text { c } & -2.14851 & -5.00924 & 2.66721\end{array}$

C $\quad-1.89918-3.63566 \quad 2.73130$

c $\quad-1.65040-2.90875 \quad 1.54994$

$\begin{array}{llll}\text { C } & -1.64720 & -3.55081 & 0.29474\end{array}$

$\begin{array}{llll}\text { C } & -1.89914 & -4.92595 & 0.23229\end{array}$

$\begin{array}{llll}0 & -1.35628 & -1.57319 & 1.72343\end{array}$

$\begin{array}{llll}\text { C } & -1.80010 & -0.61023 & 0.81182\end{array}$

$\begin{array}{lllll}0 & -2.51848 & -0.83907 & -0.13333\end{array}$

h $\quad-1.45319-2.98016-0.62255$

$\begin{array}{llll}\mathrm{n} & -1.26142 & 0.59182 & 1.22173\end{array}$

c $\quad-1.32655 \quad 1.88128 \quad 0.61630$

$\begin{array}{llll}0 & -0.37621 & 2.63403 & 0.80765\end{array}$

$\begin{array}{llll}\text { C } & -2.50438 & 2.27650 & -0.18673\end{array}$

$\begin{array}{llll}\text { C } & -3.78062 & 1.86152 & 0.06157\end{array}$

$\begin{array}{llll}0 & -4.14100 & 1.05298 & 1.06963\end{array}$

$\begin{array}{llll}\text { C } & -5.41170 & 0.39348 & 0.94285\end{array}$

$\begin{array}{llll}\text { C } & -5.74500 & -0.26778 & 2.26696\end{array}$

$\begin{array}{llll}\text { c } & -2.24246 & 3.35592 & -1.21031\end{array}$

$\begin{array}{llll}\text { h } & -0.51061 & 0.52959 & 1.91879\end{array}$

h $\quad-3.17016 \quad 3.62558-1.75824$

h $\quad-1.48100 \quad 3.03049 \quad-1.95374$

$\begin{array}{lllll}\text { h } & -1.83967 & 4.27142 & -0.72375\end{array}$

h $\quad-4.61419 \quad 2.24285-0.56482$

$\begin{array}{llll}\text { h } & -6.18616 & 1.14690 & 0.66027\end{array}$

$\begin{array}{llll}\text { h } & -5.33996 & -0.35944 & 0.12515\end{array}$

h $\quad \begin{array}{llll}-6.72982 & -0.77794 & 2.19848\end{array}$

h $\quad-4.97883 \quad-1.02804 \quad 2.53162$

$\begin{array}{llll}\text { h } & -5.79363 & 0.48257 & 3.08537\end{array}$

h $\quad-1.90223 \quad-5.46482-0.72608$

$\begin{array}{llll}\text { n } & -2.41714 & -7.09372 & 1.33760\end{array}$

h $\quad-2.34679 \quad-5.60621 \quad 3.56871$

h $\quad-1.88924 \quad-3.10217 \quad 3.69413$

$\begin{array}{lllll}0 & -2.40297 & -7.61521 & 0.22219\end{array}$

$\begin{array}{llll}0 & -2.63687 & -7.68532 & 2.39512\end{array}$

http://mc.manuscriptcentral.com/poc 
2NH-ZEZE

$E(B 3 L Y P /$ def2-TZVP $)=-1064.425966201$

35

C $\quad-2.67454-5.29652 \quad 1.75000$

$\begin{array}{llll}\text { C } & -1.70755 & -4.69910 & 2.57439\end{array}$

$\begin{array}{llll}\text { C } & -1.23401 & -3.42937 & 2.24352\end{array}$

C $\quad-1.72732-2.76598 \quad 1.09892$

$\begin{array}{llll}\text { C } & -2.69879 & -3.37124 & 0.27359\end{array}$

C $\quad-3.16856 \quad-4.64740 \quad 0.61099$

$\begin{array}{lllll}0 & -1.15687 & -1.52676 & 0.90933\end{array}$

C $\quad-1.48553-0.65019-0.08111$

$\begin{array}{lllll}0 & -2.32558 & -0.81476 & -0.94659\end{array}$

h $\quad-3.07517 \quad-2.84549-0.61133$

$\begin{array}{llll}\mathrm{n} & -0.72728 & 0.51042 & 0.00193\end{array}$

$\begin{array}{llll}\text { C } & 0.29020 & 0.90428 & 0.90232\end{array}$

$\begin{array}{lllll}0 & 0.68164 & 0.21128 & 1.83175\end{array}$

$\begin{array}{llll}\text { C } & 0.88487 & 2.25450 & 0.64261\end{array}$

c $\quad 0.52822 \quad 3.12305-0.34744$

$\begin{array}{lllll}0 & -0.46211 & 2.90568 & -1.24734\end{array}$

c $\quad-0.59330 \quad 3.83960 \quad-2.33575$

$\begin{array}{llll}\text { C } & -1.87256 & 3.52492 & -3.08790\end{array}$

C $\quad 1.98035 \quad 2.637281 .60659$

h $\quad-0.96866 \quad 1.18744 \quad-0.73504$

h $\quad 2.40061 \quad 3.63830 \quad 1.37151$

$\begin{array}{llll}\text { h } & 2.80469 & 1.89095 & 1.58542\end{array}$

$\begin{array}{llll}\text { h } & 1.60159 & 2.64791 & 2.65218\end{array}$

h $\quad \begin{array}{llll}1.04791 & 4.09580 & -0.45087\end{array}$

h $\quad-0.61165 \quad 4.87409-1.91973$

h $\quad 0.29918 \quad 3.74750-2.99779$

h $\quad-1.98914 \quad 4.22654-3.94135$

h $\quad \begin{array}{llll}-1.85399 & 2.48887 & -3.48942\end{array}$

$\begin{array}{lllll}\text { h } & -2.75863 & 3.62808 & -2.42569\end{array}$

h $\quad-3.92437-5.15601-0.00451$

$\begin{array}{llll}\mathrm{n} & -3.18316 & -6.64361 & 2.09262\end{array}$

h $\quad-1.34514 \quad-5.24127 \quad 3.45925$

h $\quad-0.47532 \quad-2.91482 \quad 2.85266$

$\begin{array}{lllll}\text { o } & -4.02564 & -7.14070 & 1.34368\end{array}$

$\begin{array}{llll}0 & -2.73100 & -7.18082 & 3.10493\end{array}$

\section{NH-ZEZZ}

$E(B 3 L Y P / d e f 2-T Z V P)=-1064.424564486$

35

$\begin{array}{llll}\text { c } & -3.54689 & -4.32630 & 1.41539 \\ \text { c } & -3.16736 & -3.03066 & 1.04284 \\ c & -1.81680 & -2.78152 & 0.71386 \\ c & -0.86500 & -3.82484 & 0.75975 \\ \text { c } & -1.24847 & -5.11344 & 1.13236 \\ c & -2.59272 & -5.35159 & 1.45716 \\ \text { o } & -1.28900 & -1.56073 & 0.36313 \\ \text { c } & -2.05374 & -0.43347 & 0.02420\end{array}$

http://mc.manuscriptcentral.com/poc 
n $\quad-1.13422 \quad 0.56876-0.22590$

c $\quad-1.43433 \quad 1.90205-0.58796$

C $\quad-0.24993 \quad 2.79617 \quad-0.78908$

C $\quad-0.60750 \quad 4.20919-1.17608$

h $\quad 0.17965-3.59670 \quad 0.49691$

o $\quad-3.25670 \quad-0.38844-0.03062$

$\begin{array}{lllll}0 & -2.57635 & 2.31262 & -0.72486\end{array}$

c $\quad 1.060622 .44525 \quad-0.65204$

$\begin{array}{lllll}0 & 1.50061 & 1.19659 & -0.35151\end{array}$

$\begin{array}{llll}\text { C } & 2.88853 & 1.05058 & 0.00041\end{array}$

$\begin{array}{llll}\text { C } & 3.21509 & -0.42930 & 0.07095\end{array}$

h $\quad-0.13948 \quad 0.31983-0.13793$

h $\quad 0.29753 \quad 4.84170-1.29669$

h $\quad-1.26816 \quad 4.67249-0.41081$

$\begin{array}{llll}\text { h } & -1.18175 & 4.22549 & -2.12854\end{array}$

h $\quad \begin{array}{llll}1.86070 & 3.19535 & -0.80866\end{array}$

h $\quad 3.50991 \quad 1.56536-0.76933$

$\begin{array}{llll}\text { h } & 3.06681 & 1.55166 & 0.98056\end{array}$

$\begin{array}{llll}\text { h } & 4.28061 & -0.56738 & 0.35351\end{array}$

h $\quad 2.58616-0.93934 \quad 0.83256$

h $\quad 3.04696-0.92044-0.91150$

h $\quad-0.52990 \quad-5.94396 \quad 1.17678$

$\begin{array}{llll}\mathrm{n} & -3.00793 & -6.71527 & 1.85392\end{array}$

h $\quad-4.58905 \quad-4.55748 \quad 1.67939$

h $\quad-3.90502 \quad-2.22141 \quad 0.99783$

$\begin{array}{llll}\text { o } & -4.19467 & -6.89062 & 2.13171\end{array}$

$\begin{array}{llll}0 & -2.13732 & -7.58727 & 1.88106\end{array}$

\section{NH-ZZEE}

$E(B 3 L Y P /$ def2-TZVP $)=-1064.413364898$

35

C $\quad-2.47449 \quad-4.27795 \quad 2.02136$

$\begin{array}{llll}\text { C } & -3.77146 & -4.00622 & 1.56461\end{array}$

C $\quad-4.06265-2.853190 .82066$

$\begin{array}{llll}\text { C } & -3.02979 & -1.95768 & 0.53526\end{array}$

c $\quad-1.72212 \quad-2.22125 \quad 0.99104$

c $\quad-1.43607 \quad-3.38315 \quad 1.73668$

$\begin{array}{lllll}0 & -0.77975 & -1.29566 & 0.59162\end{array}$

$\begin{array}{llll}\text { C } & 0.37950 & -1.05490 & 1.29984\end{array}$

$\begin{array}{llll}\mathrm{n} & 1.17997 & -0.15050 & 0.62080\end{array}$

$\begin{array}{llll}\text { c } & 0.95593 & 0.64484 & -0.55778\end{array}$

$\begin{array}{llll}\text { C } & 0.37256 & -0.03241 & -1.74497\end{array}$

c $\quad 0.74137-1.46519-2.06154$

h $\quad-0.41897 \quad-3.57664 \quad 2.09838$

$\begin{array}{lllll}0 & 0.65206 & -1.52746 & 2.38291\end{array}$

$\begin{array}{lllll}0 & 1.36102 & 1.79758 & -0.53626\end{array}$

$\begin{array}{llll}\text { c } & -0.39196 & 0.68051 & -2.62473\end{array}$

$\begin{array}{lllll}0 & -0.80431 & 1.93913 & -2.42644\end{array}$

C $\quad-1.47086 \quad 2.58495-3.52044$

$\begin{array}{llll}\text { c } & -2.02530 & 3.90850 & -3.02720\end{array}$

$\begin{array}{llll}\text { h } & 1.88374 & 0.27165 & 1.23829\end{array}$ 
h $\quad 0.40592 \quad-1.73515-3.08619$

h $\quad 0.28266-2.19681-1.36156$

h $\quad 1.84365-1.61618-2.02177$

h $\quad-0.74953 \quad 0.19596-3.55768$

h $\quad-0.74140 \quad 2.74281-4.34959$

h $\quad-2.28550 \quad 1.92259-3.90223$

h $\quad-2.53309 \quad 4.44204-3.85917$

h $\quad-2.75972 \quad 3.74907-2.20895$

h $\quad-1.20803 \quad 4.55378-2.64046$

h $\quad-2.29392 \quad-5.19607 \quad 2.59855$

$\begin{array}{llll}\mathrm{n} & -4.86207 & -4.96054 & 1.87172\end{array}$

h $\quad-5.09358 \quad-2.678830 .48177$

h $\quad-3.21169-1.04008-0.04461$

$\begin{array}{llll}\text { o } & -4.57259 & -5.96136 & 2.52765\end{array}$

$\begin{array}{lllll}0 & -5.98686 & -4.68976 & 1.44883\end{array}$

\section{NH-ZZEZ}

$E(B 3 L Y P /$ def2-TZVP $)=-1064.417116111$

35

$\begin{array}{llll}\text { C } & -1.57841 & -4.56378 & -0.28301\end{array}$

$\begin{array}{llll}\text { C } & -1.82004 & -5.45166 & 0.77458\end{array}$

$\begin{array}{llll}\text { C } & -2.18036 & -4.99477 & 2.05088\end{array}$

$\begin{array}{llll}\text { c } & -2.30519 & -3.61976 & 2.26347\end{array}$

$\begin{array}{llll}\text { c } & -2.06857 & -2.71815 & 1.20634\end{array}$

C $\quad-1.70122 \quad-3.18557-0.07223$

$\begin{array}{llll}\text { o } & -2.16128 & -1.38354 & 1.54162\end{array}$

$\begin{array}{llll}\text { C } & -2.60241 & -0.42210 & 0.63167\end{array}$

$\begin{array}{llll}\mathrm{n} & -2.49620 & 0.80056 & 1.26233\end{array}$

$\begin{array}{llll}\text { c } & -2.59900 & 2.12104 & 0.69744\end{array}$

$\begin{array}{llll}\text { C } & -3.65271 & 2.36476 & -0.31351\end{array}$

C $\quad-4.97288 \quad 1.62644-0.26479$

h $\quad-1.52094-2.48028-0.89310$

$\begin{array}{lllll}0 & -3.00162 & -0.65817 & -0.48698\end{array}$

$\begin{array}{lllll}0 & -1.84594 & 2.96887 & 1.15653\end{array}$

C $\quad-3.47331 \quad 3.35478-1.24185$

$\begin{array}{lllll}0 & -2.36507 & 4.09126 & -1.37671\end{array}$

C $\quad-2.31722 \quad 4.98763-2.49735$

c $\quad-1.049925 .81488-2.39139$

$\begin{array}{lllll}\text { h } & -1.93587 & 0.80094 & 2.12283\end{array}$

$\begin{array}{llll}\text { h } & -5.71197 & 2.11084 & -0.93901\end{array}$

$\begin{array}{llll}\text { h } & -5.40447 & 1.63841 & 0.76172\end{array}$

h $\quad-4.869290 .56576-0.57790$

h $\quad-4.28603 \quad 3.56905-1.96665$

h $\quad-2.33146 \quad 4.39423-3.44262$

h $\quad-3.22390 \quad 5.63871-2.48735$

h $\quad \begin{array}{llll}-0.97971 & 6.51537 & -3.25117\end{array}$

h $\quad-1.04393 \quad 6.40525-1.45051$

h $\quad-0.152345 .16036-2.39584$

h $\quad-1.29274-4.96873-1.26431$

$\begin{array}{llll}\mathrm{n} & -1.68896 & -6.90840 & 0.54063\end{array}$

h $\quad-2.35961 \quad-5.72506 \quad 2.85259$

$\begin{array}{llll}\text { h } & -2.58607 & -3.21864 & 3.24918\end{array}$

http://mc.manuscriptcentral.com/poc 
\begin{tabular}{lllll}
\hline & & -1.35720 & -7.27431 & -0.58755 \\
0 & & -1.92035 & -7.65769 & 1.49024
\end{tabular}

2NH-ZZZE

$E(B 3 L Y P /$ def2-TZVP $)=-1064.417115758$

35

C $\quad-3.30666 \quad-4.79803 \quad 0.84140$

$\begin{array}{llll}\text { C } & -2.75895 & -5.29521 & 2.03157\end{array}$

C $\quad-1.65875 \quad-4.67706 \quad 2.64755$

c $\quad-1.10442 \quad-3.543092 .05316$

$\begin{array}{llll}\text { c } & -1.65063 & -3.03370 & 0.85461\end{array}$

C $\quad-2.75693 \quad-3.657810 .24000$

$\begin{array}{lllll}0 & -0.98648 & -1.91798 & 0.39486\end{array}$

C $\quad-1.32402-1.19779-0.70886$

$\begin{array}{lllll}\mathrm{n} & -0.43799 & -0.14406 & -0.90671\end{array}$

c $\quad 0.73617 \quad 0.25744 \quad-0.19196$

C $\quad 1.37269 \quad 1.47230-0.77526$

C $\quad 0.76862 \quad 2.19915-1.95829$

h $\quad-3.17370-3.25131-0.68861$

o $\quad-2.26631 \quad-1.40241-1.45184$

$\begin{array}{llll}0 & 1.14818 & -0.34849 & 0.78355\end{array}$

C $\quad 2.53551 \quad 1.96283 \quad-0.24036$

$\begin{array}{lllll}0 & 3.19794 & 1.44187 & 0.79312\end{array}$

C $\quad 4.401592 .11776 \quad 1.19608$

$\begin{array}{llll}\text { C } & 5.01077 & 1.34876 & 2.35311\end{array}$

h $\quad-0.705850 .39998-1.73066$

h $\quad \begin{array}{lllll}1.37486 & 3.09000 & -2.22976\end{array}$

h $\quad-0.26275 \quad 2.56854-1.74924$

h $\quad 0.71922 \quad 1.56189-2.87301$

$\begin{array}{llll}\text { h } & 2.98891 & 2.87072 & -0.69061\end{array}$

h $\quad 5.10381 \quad 2.16531 \quad 0.32984$

h $\quad 4.15170 \quad 3.16346 \quad 1.49483$

$\begin{array}{llll}\text { h } & 5.94469 & 1.84684 & 2.69097\end{array}$

h $\quad 4.30381 \quad 1.30297 \quad 3.20850$

$\begin{array}{llll}\text { h } & 5.25363 & 0.30874 & 2.04822\end{array}$

h $\quad-4.16776-5.31556 \quad 0.39496$

$\begin{array}{llll}\text { n } & -3.35214 & -6.49979 & 2.65395\end{array}$

h $\quad-1.25823 \quad-5.09819 \quad 3.58026$

h $\quad-0.23942 \quad-3.02141 \quad 2.48997$

$\begin{array}{lllll}\text { o } & -4.30837 & -7.02425 & 2.08091\end{array}$

$\begin{array}{lllll}0 & -2.85032 & -6.90033 & 3.70553\end{array}$

2NH-ZZZZ

$E(B 3 L Y P /$ def2-TZVP $)=-1064.416225655$

35

C $\quad-2.76742 \quad-5.25911 \quad 1.46419$

c $\quad-3.60431-4.141621 .58909$

$\begin{array}{llll}\text { C } & -3.12749 & -2.87230 & 1.24093\end{array}$

http://mc.manuscriptcentral.com/poc 
C $\quad-1.80446 \quad-2.74333 \quad 0.76660$

$\begin{array}{llll}\text { c } & -0.97182 & -3.87609 & 0.64502\end{array}$

C $\quad-1.45015 \quad-5.140120 .99598$

$\begin{array}{llll}0 & -1.20310 & -1.54653 & 0.44816\end{array}$

$\begin{array}{llll}\text { C } & -1.92985 & -0.42399 & 0.01250\end{array}$

$\begin{array}{lllll}0 & -3.11492 & -0.39964 & -0.19749\end{array}$

$\begin{array}{llll}\text { h } & 0.05514 & -3.74038 & 0.27257\end{array}$

$\begin{array}{lllll}\mathrm{n} & -0.99999 & 0.59175 & -0.12070\end{array}$

c $\quad-1.27708 \quad 1.93562-0.52938$

$\begin{array}{lllll}0 & -2.40627 & 2.29510 & -0.81079\end{array}$

C $\quad-0.060552 .79368-0.56122$

C $\quad-0.15764 \quad 4.10813-0.93787$

$\begin{array}{lllll}0 & -1.28102 & 4.73063 & -1.29418\end{array}$

c $\quad-1.16787 \quad 6.11395-1.67028$

$\begin{array}{llll}\text { c } & -2.55439 & 6.62302 & -2.01626\end{array}$

$\begin{array}{llll}\text { c } & 1.30237 & 2.25270 & -0.18466\end{array}$

$\begin{array}{lllll}\text { h } & -0.03804 & 0.32996 & 0.10767\end{array}$

h $\quad 2.08077 \quad 3.04116-0.26899$

h $\quad \begin{array}{llll}1.62786 & 1.41585 & -0.84726\end{array}$

$\begin{array}{llll}\text { h } & 1.33942 & 1.88322 & 0.86761\end{array}$

$\begin{array}{lllll}\text { h } & 0.76264 & 4.72989 & -0.95531\end{array}$

h $\quad-0.72672 \quad 6.68972-0.82213$

$\begin{array}{lllll}\text { h } & -0.47749 & 6.20087 & -2.54269\end{array}$

h $\quad-2.50328 \quad 7.69221 \quad-2.31421$

h $\quad-2.98711 \quad 6.04022 \quad-2.85682$

h $\quad-3.23532 \quad 6.52905-1.14390$

$\begin{array}{llll}\text { h } & -0.82631 & -6.04121 & 0.91304\end{array}$

n $\quad-3.28166 \quad-6.59575 \quad 1.83850$

h $\quad-4.62846 \quad-4.28142 \quad 1.96389$

h $\quad-3.77616 \quad-1.99243 \quad 1.32612$

$\begin{array}{llll}\text { o } & -2.51497 & -7.55256 & 1.71787\end{array}$

$\begin{array}{llll}0 & -4.44154 & -6.66439 & 2.24714\end{array}$

\section{OH-EEZE}

$E(B 3 L Y P /$ def2-TZVP $)=-1064.409859500$

35

$\begin{array}{llll}\text { C } & 0.07412 & 0.04846 & 1.84705\end{array}$

$\begin{array}{llll}\text { C } & 1.13660 & -0.55077 & 2.55492\end{array}$

c $\quad 1.10231-0.61268 \quad 3.95052$

$\begin{array}{llll}\text { C } & -0.00662 & -0.07968 & 4.62387\end{array}$

$\begin{array}{llll}\text { C } & -1.07058 & 0.51622 & 3.93143\end{array}$

$\begin{array}{llll}\text { c } & -1.03439 & 0.58522 & 2.53448\end{array}$

$\begin{array}{llll}\mathrm{n} & -0.05241 & -0.14659 & 6.10223\end{array}$

$\begin{array}{lllll}0 & -1.04005 & 0.33057 & 6.66228\end{array}$

$\begin{array}{lllll}0 & 0.21309 & 0.12028 & 0.47906\end{array}$

$\begin{array}{llll}\text { C } & -0.92103 & -0.10366 & -0.45031\end{array}$

n $\quad-0.55054 \quad-0.01551-1.74544$

$\begin{array}{llll}\text { c } & 0.65704 & 0.23325 & -2.22414\end{array}$

$\begin{array}{llll}\text { c } & 0.89747 & 0.27533 & -3.66331\end{array}$

C $\quad 2.278620 .57026-4.18480$

$\begin{array}{llll}0 & 0.90120 & -0.67499 & 6.67598\end{array}$

$\begin{array}{llll}0 & -2.01417 & -0.34043 & 0.00447\end{array}$ 
$\begin{array}{llll}0 & 1.75504 & 0.46538 & -1.49525\end{array}$

$\begin{array}{lllll}\text { C } & -0.17338 & 0.03210 & -4.48324\end{array}$

$\begin{array}{lllll}0 & -0.06698 & 0.04138 & -5.81455\end{array}$

$\begin{array}{llll}\text { C } & -1.26345 & -0.24662 & -6.56592\end{array}$

$\begin{array}{llll}\text { C } & -0.92654 & -0.17958 & -8.04305\end{array}$

h $\quad \begin{array}{llll}1.98623 & -0.96930 & 1.99275\end{array}$

h $\quad-1.16592 \quad-0.18100-4.04297$

h $\quad-1.63350-1.25856-6.28035$

$\begin{array}{lllll}\text { h } & -2.04786 & 0.49741 & -6.29481\end{array}$

h $\quad-1.83269-0.39959-8.64710$

h $\quad-0.55818 \quad 0.83135-8.32002$

h $\quad-0.14265-0.92230-8.30440$

h $\quad 1.91432 \quad-1.07208 \quad 4.53182$

$\begin{array}{llll}\text { h } & -1.91658 & 0.92416 & 4.50294\end{array}$

$\begin{array}{llll}\text { h } & -1.86019 & 1.04442 & 1.97692\end{array}$

h $\quad \begin{array}{llll}1.47310 & 0.40150 & -0.52941\end{array}$

h $\quad 2.28644 \quad 0.56138 \quad-5.29318$

$\begin{array}{llll}\text { h } & 2.63738 & 1.56377 & -3.83571\end{array}$

$\begin{array}{llll}\text { h } & 3.01650 & -0.17796 & -3.81924\end{array}$

3OH-EZZE

$E(B 3 L Y P / d e f 2-T Z V P)=-1064.408934712$

35

C $\quad 1.43774 \quad-0.22588 \quad 3.81820$

$\begin{array}{llll}\text { C } & 0.40274 & 0.22328 & 4.65131\end{array}$

C $\quad-0.85194 \quad 0.58143 \quad 4.13730$

$\begin{array}{llll}\text { C } & -1.08667 & 0.49301 & 2.76084\end{array}$

$\begin{array}{llll}\text { C } & -0.05393 & 0.03857 & 1.91432\end{array}$

$\begin{array}{llll}\text { C } & 1.20226 & -0.32279 & 2.44434\end{array}$

$\begin{array}{llll}\mathrm{n} & 0.64363 & 0.32430 & 6.10882\end{array}$

$\begin{array}{lllll}0 & -0.28864 & 0.71390 & 6.81270\end{array}$

$\begin{array}{lllll}0 & -0.18143 & -0.02748 & 0.54457\end{array}$

$\begin{array}{lllll}0 & & 1.76180 & 0.01269 & 6.52164\end{array}$

C $\quad-1.42369-0.48139-0.12276$

n $\quad-1.32701-0.45719-1.47323$

$\begin{array}{llll}\text { c } & -0.25817 & -0.17542 & -2.19407\end{array}$

$\begin{array}{llll}\text { c } & -0.38104 & -0.20709 & -3.64774\end{array}$

$\begin{array}{llll}\text { C } & -1.70738 & -0.56787 & -4.26043\end{array}$

$\begin{array}{lllll}0 & -2.36557 & -0.81463 & 0.55271\end{array}$

$\begin{array}{lllll}0 & 0.95500 & 0.13577 & -1.70931\end{array}$

C $\quad 0.72547 \quad 0.09606-4.39669$

$\begin{array}{lllll}0 & 0.70282 & 0.08922 & -5.73475\end{array}$

$\begin{array}{llll}\text { C } & 1.92700 & 0.43275 & -6.41036\end{array}$

$\begin{array}{lllll}\text { C } & 1.68814 & 0.34381 & -7.90555\end{array}$

$\begin{array}{llll}\text { h } & 1.98862 & -0.68232 & 1.76223\end{array}$

h $\quad \begin{array}{llll}\text { h } & 1.68489 & 0.35868 & -3.91404\end{array}$

$\begin{array}{lllll}\text { h } & 2.22960 & 1.46324 & -6.11034\end{array}$

$\begin{array}{llll}\text { h } & 2.72986 & -0.27076 & -6.08745\end{array}$

$\begin{array}{lllll}\text { h } & 2.61831 & 0.60661 & -8.45347\end{array}$

h $\quad \begin{array}{llll}1.38773 & -0.68517 & -8.19739\end{array}$

h $\quad \begin{array}{llll}0.88595 & 1.04522 & -8.21997\end{array}$

h $\quad 2.40589 \quad-0.49699 \quad 4.26244$ 
h $\quad \begin{array}{llll}-1.63078 & 0.93154 & 4.82963\end{array}$

$\begin{array}{llll}\text { h } & -2.06363 & 0.76740 & 2.34377\end{array}$

h $\quad 0.86152 \quad 0.15211 \quad-0.70697$

h $\quad-1.64312 \quad-0.56259-5.36694$

h $\quad-2.03978-1.57221-3.91987$

h $\quad-2.49902 \quad 0.14404 \quad-3.94036$

Transition state for the direct 2NH to Phenol Hydrogen Transfer $E(B 3 L Y P / d e f 2-T Z V P)=-1064.358055726$

35

$\begin{array}{llll}\text { C } & -2.40239 & -4.39409 & 2.02309\end{array}$

C $\quad-1.73785 \quad-3.23073 \quad 2.44622$

$\begin{array}{llll}\text { C } & -1.06939 & -2.44412 & 1.50734\end{array}$

$\begin{array}{llll}\text { C } & -1.04986 & -2.81312 & 0.13301\end{array}$

$\begin{array}{llll}\text { C } & -1.72300 & -4.00159 & -0.26578\end{array}$

$\begin{array}{llll}\text { C } & -2.39817 & -4.78273 & 0.67158\end{array}$

$\begin{array}{lllll}0 & -0.40429 & -2.07096 & -0.76627\end{array}$

C $\quad-1.35411-1.08888-2.36933$

o $\quad-1.89157-1.72774-3.18496$

n $\quad-3.11322 \quad-5.21902 \quad 3.01156$

$\begin{array}{llll}0 & -3.68887 & -6.23255 & 2.60511\end{array}$

n $\quad-0.89523 \quad-0.07151-1.71647$

c $\quad-1.06614 \quad 1.31328-2.14381$

$\begin{array}{lllll}0 & -1.67866 & 1.56157 & -3.16948\end{array}$

$\begin{array}{llll}\text { C } & -0.44273 & 2.26698 & -1.21575\end{array}$

C $\quad-0.58970 \quad 3.58169 \quad-1.57271$

$\begin{array}{lllll}0 & -0.09056 & 4.58380 & -0.84940\end{array}$

C $\quad-0.31713 \quad 5.92323-1.34107$

c $\quad 0.33073 \quad 6.90055-0.38033$

$\begin{array}{llll}\text { C } & 0.29424 & 1.83379 & 0.02508\end{array}$

o $\quad-3.09272-4.84796 \quad 4.18910$

h $\quad-1.69941-4.29093-1.32811$

h $\quad-0.41574 \quad-0.72228-0.82918$

$\begin{array}{llll}\text { h } & 0.69550 & 2.71764 & 0.56096\end{array}$

h $\quad \begin{array}{llll}1.15064 & 1.16380 & -0.21366\end{array}$

$\begin{array}{lllll}\text { h } & -0.36908 & 1.28332 & 0.73049\end{array}$

h $\quad-1.14305 \quad 3.83978-2.49653$

h $\quad 0.11945 \quad 6.00812-2.36301$

h $\quad-1.41543 \quad 6.09561 \quad-1.41688$

h $\quad 0.16964 \quad 7.94028-0.73687$

$\begin{array}{llll}\text { h } & -0.10801 & 6.80753 & 0.63604\end{array}$

$\begin{array}{lllll}\text { h } & 1.42468 & 6.72060 & -0.30934\end{array}$

$\begin{array}{llll}\text { h } & -2.92817 & -5.70207 & 0.38303\end{array}$

h $\quad-1.76279-2.96383 \quad 3.51271$

h $\quad-0.53555 \quad-1.53160 \quad 1.82052$

Transition State for EEZE isomer

$E(B 3 L Y P /$ def2-TZVP) $=-1064.397785941$ 
$\begin{array}{llll}\text { C } & 1.20004 & -0.40966 & 3.63584\end{array}$

c $\quad 0.10496-0.18106 \quad 4.48493$

$\begin{array}{llll}\text { C } & -1.06649 & 0.43730 & 4.01933\end{array}$

$\begin{array}{llll}\text { C } & -1.14452 & 0.84180 & 2.68442\end{array}$

$\begin{array}{llll}\text { C } & -0.05231 & 0.61956 & 1.81088\end{array}$

C $\quad 1.11745 \quad-0.015742 .29882$

$\begin{array}{llll}\text { n } & 0.18915 & -0.59921 & 5.89777\end{array}$

$\begin{array}{lllll}0 & -0.79004 & -0.38507 & 6.61598\end{array}$

$\begin{array}{lllll}0 & -0.11891 & 1.02808 & 0.52446\end{array}$

$\begin{array}{lllll}0 & 1.23402 & -1.13710 & 6.27246\end{array}$

$\begin{array}{llll}\text { C } & -1.35191 & 0.25102 & -0.60013\end{array}$

$\begin{array}{lllll}\mathrm{n} & -0.80260 & 0.16398 & -1.76974\end{array}$

c $\quad 0.48437 \quad 0.39581-2.12520$

c $\quad 0.88748 \quad 0.18007-3.50585$

C $\quad 2.31413 \quad 0.45151-3.90011$

$\begin{array}{lllll}0 & -2.35937 & 0.02887 & -0.01530\end{array}$

$\begin{array}{lllll}0 & 1.39933 & 0.80498 & -1.30241\end{array}$

$\begin{array}{llll}\text { c } & -0.06719 & -0.25784 & -4.38956\end{array}$

o $\quad 0.19875-0.45544-5.67889$

c $\quad-0.83889-1.01978-6.50806$

$\begin{array}{llll}\text { c } & -0.60725 & -0.58402 & -7.94246\end{array}$

h $\quad \begin{array}{llll}1.95995 & -0.19202 & 1.61085\end{array}$

h $\quad-1.10096-0.44914-4.04334$

h $\quad-0.79251-2.12881-6.40965$

h $\quad-1.83139-0.67995-6.13329$

h $\quad-1.37352-1.04300-8.60340$

$\begin{array}{llll}\text { h } & -0.67499 & 0.52068 & -8.03992\end{array}$

h $\quad 0.39500 \quad-0.90739-8.29599$

h $\quad 2.09701 \quad-0.89851 \quad 4.04226$

$\begin{array}{llll}\text { h } & -1.89700 & 0.59896 & 4.72145\end{array}$

h $\quad-2.04471 \quad 1.33845 \quad 2.29577$

h $\quad 0.87181 \quad 0.95255-0.30500$

$\begin{array}{llll}\text { h } & 2.48132 & 0.19892 & -4.96608\end{array}$

h $\quad 2.57561 \quad 1.52159-3.74341$

h $\quad 3.01976-0.13935-3.27606$

Transition State for EZZE isomer $E(B 3 L Y P /$ def2-TZVP $)=-1064.396547491$

35

$\begin{array}{llll}\text { C } & 0.61848 & -0.05891 & 4.30963\end{array}$

$\begin{array}{llll}\text { C } & -0.70152 & 0.38973 & 4.14185\end{array}$

$\begin{array}{llll}\text { C } & -1.15657 & 0.72160 & 2.86347\end{array}$

$\begin{array}{llll}\text { c } & -0.29295 & 0.59542 & 1.74769\end{array}$

$\begin{array}{llll}\text { C } & 1.03341 & 0.13159 & 1.93889\end{array}$

C $\quad 1.49254-0.18757 \quad 3.21806$

$\begin{array}{lllll}0 & -0.72486 & 0.92869 & 0.51190\end{array}$

$\begin{array}{llll}\mathrm{n} & 1.10021 & -0.40085 & 5.66197\end{array}$

$\begin{array}{llll}0 & 2.26852 & -0.78100 & 5.77241\end{array}$

$\begin{array}{lllll}0 & 0.30528 & -0.28609 & 6.59739\end{array}$

c $\quad-2.09027-0.05994-0.25973$

$\begin{array}{lllll}0 & -2.91537 & -0.31357 & 0.55103\end{array}$

$\begin{array}{llll}\mathrm{n} & -1.80136 & -0.20702 & -1.51548\end{array}$ 
Product Complex EEZE

$E(B 3 L Y P /$ def2-TZVP) $=-1064.425976133$

35

$\begin{array}{llll}\text { C } & -0.86892 & 0.73392 & 2.10624\end{array}$

C $\quad 0.54963 \quad 0.699592 .06736$

C $\quad 1.27408 \quad 0.28159 \quad 3.18531$

$\begin{array}{llll}\text { c } & 0.58880 & -0.10284 & 4.34941\end{array}$

$\begin{array}{llll}\text { c } & -0.81617 & -0.07204 & 4.40881\end{array}$

$\begin{array}{llll}\text { C } & -1.54001 & 0.34437 & 3.29278\end{array}$

$\begin{array}{llll}\mathrm{n} & 1.35357 & -0.54439 & 5.52483\end{array}$

$\begin{array}{llll}0 & 0.71913 & -0.86949 & 6.53270\end{array}$

$\begin{array}{llll}0 & -1.61598 & 1.12891 & 1.06047\end{array}$

$\begin{array}{llll}0 & 2.58499 & -0.56470 & 5.43426\end{array}$

$\begin{array}{lllll}0 & -0.17887 & 1.43195 & -1.15164\end{array}$

c $\quad-0.39248 \quad 0.64736-2.08926$

$\begin{array}{lllll}\text { c } & 0.39453 & 0.68477 & -3.31804\end{array}$

$\begin{array}{llll}\text { C } & 1.48828 & 1.71258 & -3.41255\end{array}$

n $\quad-1.40834-0.32786-2.03560$

c $\quad-2.26510-0.72664-1.25131$

o $\quad-3.12623-1.21979-0.62421$

c $\quad 0.11339-0.20818-4.31998$

$\begin{array}{lllll}0 & 0.80041 & -0.23437 & -5.46218\end{array}$

C $\quad 0.38270-1.18935-6.46190$

c $\quad 1.35307-1.11840-7.62460$

h $\quad \begin{array}{llll}1.07276 & 1.00910 & 1.14802\end{array}$

h $\quad-0.70393 \quad-0.94583-4.20654$

h $\quad 0.36926-2.20599-6.00537$

h $\quad-0.65460-0.93814-6.78351$

$\begin{array}{llll}\text { h } & 1.04909 & -1.84040 & -8.41228\end{array}$ 


$\begin{array}{lrrr}\mathrm{h} & 1.36485 & -0.10023 & -8.06886 \\ \mathrm{~h} & 2.38399 & -1.37106 & -7.29658 \\ \mathrm{~h} & 2.37301 & 0.24627 & 3.18019 \\ \mathrm{~h} & -1.31449 & -0.37907 & 5.33959 \\ \mathrm{~h} & -2.64034 & 0.37875 & 3.30588 \\ \mathrm{~h} & -1.03394 & 1.34633 & 0.26157 \\ \mathrm{~h} & 1.98025 & 1.67714 & -4.40470 \\ \mathrm{~h} & 1.08500 & 2.73533 & -3.24489 \\ \mathrm{~h} & 2.25838 & 1.54629 & -2.62666\end{array}$

Product Complex EEZE

$E(B 3 L Y P /$ def2-TZVP) $=-1064.424492225$

35

$\begin{array}{llll}\text { C } & 1.00777 & -0.09672 & 4.01097\end{array}$

$\begin{array}{llll}\text { C } & -0.23114 & 0.51600 & 4.27128\end{array}$

c $\quad-0.98963 \quad 1.00574 \quad 3.20932$

$\begin{array}{llll}\text { c } & -0.51995 & 0.88843 & 1.87671\end{array}$

$\begin{array}{llll}\text { C } & 0.73384 & 0.26860 & 1.63485\end{array}$

$\begin{array}{llll}\text { C } & 1.49372 & -0.22081 & 2.69903\end{array}$

$\begin{array}{lllll}0 & -1.28956 & 1.38180 & 0.89121\end{array}$

$\begin{array}{llll}\mathrm{n} & 1.80953 & -0.61330 & 5.12977\end{array}$

$\begin{array}{lllll}\text { o } & 2.89340 & -1.14197 & 4.86368\end{array}$

$\begin{array}{llll}0 & 1.35113 & -0.48835 & 6.26921\end{array}$

c $\quad-3.14334-0.26168-0.85020$

$\begin{array}{lllll}0 & -3.93351 & -0.33531 & 0.01434\end{array}$

$\begin{array}{llll}\mathrm{n} & -2.43580 & -0.27313 & -1.85317\end{array}$

$\begin{array}{llll}\text { C } & -1.15568 & 0.12464 & -2.26987\end{array}$

$\begin{array}{lllll}0 & -0.39011 & 0.76615 & -1.52828\end{array}$

$\begin{array}{llll}\text { c } & -0.83587 & -0.28464 & -3.63898\end{array}$

$\begin{array}{llll}\text { C } & 0.40200 & 0.09055 & -4.09498\end{array}$

o $\quad 0.83495-0.21182-5.31799$

$\begin{array}{llll}\text { C } & 2.15524 & 0.24817 & -5.68300\end{array}$

$\begin{array}{llll}\text { C } & 2.46559 & -0.25522 & -7.07898\end{array}$

$\begin{array}{llll}\text { h } & 1.10076 & 0.17881 & 0.59972\end{array}$

c $\quad-1.81786-1.06190-4.47504$

h $\quad \begin{array}{llll}1.08240 & 0.66462 & -3.43704\end{array}$

h $\quad 2.17025 \quad 1.36171 \quad-5.63889$

h $\quad 2.88854-0.13939-4.93872$

$\begin{array}{lllll}\text { h } & 3.48000 & 0.08078 & -7.38245\end{array}$

h $\quad 2.43947-1.36543-7.11450$

h $\quad \begin{array}{lllll}1.73181 & 0.13774 & -7.81482\end{array}$

h $\quad 2.46801 \quad-0.704392 .53820$

h $\quad \begin{array}{llll}-0.57588 & 0.59479 & 5.31232\end{array}$

h $\quad-1.96417 \quad 1.48904 \quad 3.37869$

h $\quad-0.86473 \quad 1.22270-0.01401$

h $\quad-1.36532-1.33938-5.44848$

h $\quad-2.14176-1.99241-3.95944$

h $\quad-2.73869-0.46970-4.67550$

http://mc.manuscriptcentral.com/poc 


\title{
A convenient, high-yield synthesis of 1-substituted uracil and thymine derivatives
}

\author{
Dominik Rejman*, Soňa Kovačková, Radek Pohl, Martin Dračínský, Pavel Fiedler, Ivan Rosenberg* \\ Institute of Organic Chemistry and Biochemistry, Academy of Sciences of the Czech Republic, Flemingovo nám. 2, 166 10 Prague 6, Czech Republic
}

\section{A R T I C L E I N F O}

\section{Article history:}

Received 11 May 2009

Received in revised form 24 July 2009

Accepted 7 August 2009

Available online 11 August 2009

\begin{abstract}
A B S T R A C T
Novel reagents for the synthesis of 1-substituted uracil and thymine derivatives have been developed. The aminolysis of 2- or 4-nitrophenyl 3-ethoxyacryloylcarbamate and 3-ethoxy-2-methylacryloylcarbamate with a variety of primary amino derivatives proceeded smoothly under very mild reaction conditions yielding almost quantitatively the 1,3-disubstituted urea derivatives. Their subsequent cyclization provided the 1-substituted uracil and thymine compounds, in almost quantitative yield.
\end{abstract}

(c) 2009 Elsevier Ltd. All rights reserved.

\section{Introduction}

Nucleosidation is often a crucial step in the synthesis of analogues of nucleosides. Purine nucleoside analogues are usually prepared via direct alkylation of the appropriate nucleobases (adenine, 2-amino-6-chloropurine, etc.) with the halo, tosyloxy, or mesyloxy derivatives, ${ }^{1-4}$ or under Mitsunobu conditions. ${ }^{2,3}$ However, all of these methods for the introduction of pyrimidine a competing elimination reaction which further decreases the yield of the desired compounds. As an alternative approach to the pyrimidine nucleoside analogues (e.g., carbocyclic nucleosides), a synthesis of $1-N$-substituted nucleobases starting from a primary amine and isocyanate $\mathbf{3}$ or carbamate $\mathbf{6}$ was described fifty years ago by Shaw and Warrener. ${ }^{5}$ The key isocyanates $\mathbf{3}$, accessible by two independent routes ${ }^{6,7}$ (Scheme 1), were used as intermediates for the synthesis of carbamates $\mathbf{6}$ described by Hřebabecký. ${ }^{8}$

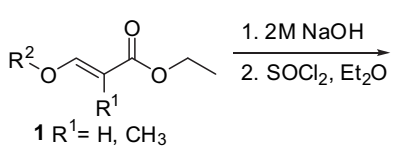

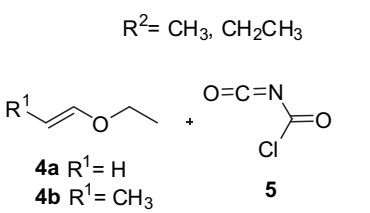

$$
\begin{aligned}
& 2 \mathrm{R}^{1}=\mathrm{H}, \mathrm{CH}_{3}
\end{aligned}
$$$$
2 \mathrm{R}^{1}=\mathrm{H}, \mathrm{CH}_{3}
$$$$
\text { 4-Nitrophenol }
$$$$
\mathrm{O}_{2}
$$<smiles>Nc1ccccc1</smiles>$$
\begin{aligned}
& 7 \text { a R } \mathrm{R}^{1}=\mathrm{H} ;(57 \%) \\
& 7 \mathbf{b ~ R}^{1}=\mathrm{CH}_{3} ;(59 \%)
\end{aligned}
$$

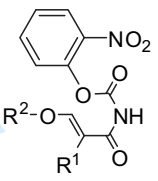<smiles>c1ccc(C23CC4CC(C2)C(C4)C3)cc1</smiles>$$
\begin{aligned}
& \text { 7c R }=\mathrm{H} ;(44 \%) \\
& \text { 7d R } \mathrm{R}^{1}=\mathrm{CH}_{3} ;(60 \%)
\end{aligned}
$$

Scheme 1.

nucleobases (uracil, thymine, and cytosine) suffer from both low regioselectivity and yield of the $1-N$-substituted product. ${ }^{2,3}$ In some cases, the direct alkylation of nucleobases is accompanied by

\footnotetext{
* Corresponding authors. Tel.: +420220183 381.

E-mail addresses: rejman@uochb.cas.cz (D. Rejman), ivan@uochb.cas.cz (I. Rosenberg).

Isocyanates $\mathbf{3}$ are extremely moisture-sensitive species and also very reactive compounds capable of forming carbamates with free hydroxy groups. ${ }^{9}$ The reaction, therefore, is usually carried out either with the appropriately protected substrate or without protection at low temperature.

The formation of uracil and thymine rings is a two-stage reaction consisting of the aminolysis of carbamate $\mathbf{6}$ or the addition of amine to isocyanate $\mathbf{3}$ followed by a cyclization of the formed acryloylurea $\mathbf{9}$ 
Journal of Physical Organic Chemistry

Page 108 of 133

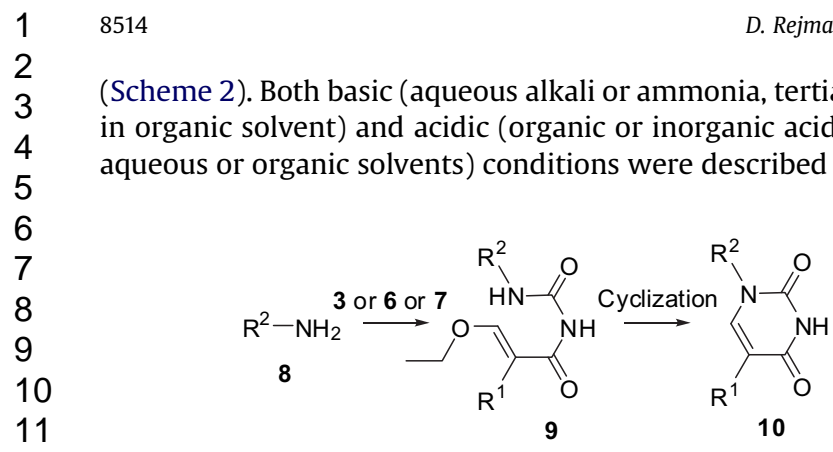

Scheme 2. clization of the urease. Best results were obtained with aqueous subfuric acid or $15 \mathrm{M}$ aqueous ammonia according to Shealy and Dell. ${ }^{10}$

Recently we described the synthesis of pyrrolidine nucleoside analogues using alkylation of purine and pyrimidine nucleobases with various pyrrolidine mesyloxy derivatives. ${ }^{2-4}$ In contrast to the purine nucleobases, the yields and regioselectivity of alkylactions of pyrimidine nucleobases are unsatisfactory. Therefore, we attempted to use Hřebabecký's ${ }^{8}$ reagents $\mathbf{6 a}$ and $\mathbf{6 b}$ for the synthesis of uracil and thymine rings to obtain pyrrolidine compounds $\mathbf{1 0 b}$ and 10n (Table 1 ), respectively. While the 'thymine reagent' $\mathbf{6 b}$ provided a moderate yield of $\mathbf{1 0 n}$, the 'uracil reagent' Ga yielded no product $\mathbf{1 0 b}$ at all. In the latter case, we only

Table 1

Synthesis of uracil and thymine derivatives using reagents $\mathbf{7 a}$ and $\mathbf{7 b}$

\begin{tabular}{lll}
\hline $\mathrm{RNH}_{2} 8$ & $9 /$ Yield$_{2} \mathrm{R}^{1}=\mathrm{H}$ & $\mathbf{1 0} /$ Yield\%
\end{tabular}

Overall yield $\mathbf{U} \% \quad \mathbf{9} /$ Yield $\% \mathrm{R}^{1}=\mathrm{CH}_{3} \quad \mathbf{1 0} /$ Yield $\%$

Overall yield $\mathbf{T} \%$

18

Bor

a

$\mathrm{NH}_{2}$

a/85

b

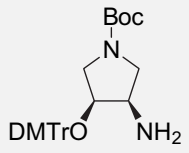

b/87

b/90
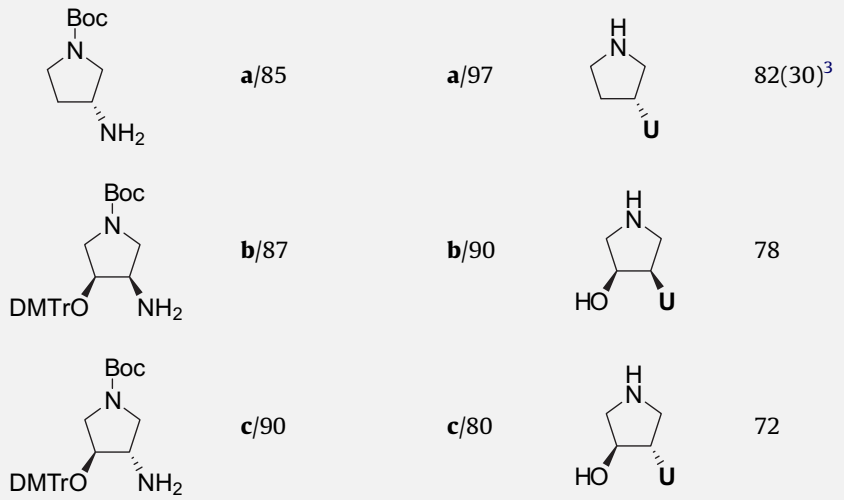

d

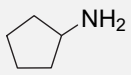

d/88

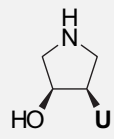

78

n/99

c/80

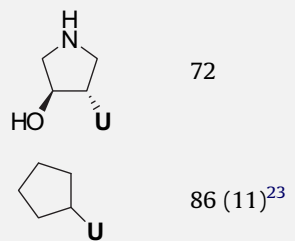

e<smiles>NC1CCCCC1</smiles>

e/88

e/99

f

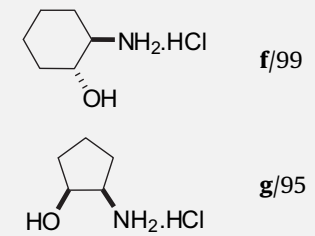

h

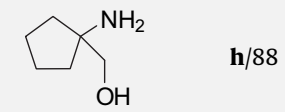

i<smiles>NCC(O)CO</smiles>

i/87

f/78

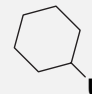

87

o/82

o/95

n/90

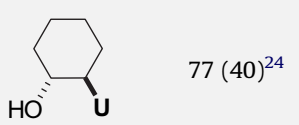

g/95

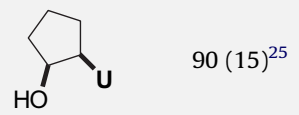

h/92

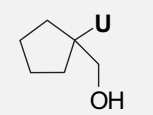

81

$\mathbf{q} / 89$

i/98

$\underbrace{\mathrm{OH}} \mathrm{U}(65)^{26}$

j/96<smiles>[AlH2]c1ccccc1</smiles>

$90(63)^{28}$

$\mathbf{s} / 87$

$\mathbf{k} / 84$

$\sum_{\mathrm{COOCH}_{3}}^{\mathbf{U}} 73(76)^{29}$

1/96

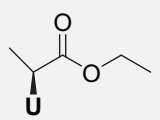

83

m

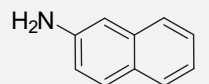

$1 / 87$<smiles>CCOC(=O)C(C)N</smiles>

$\mathbf{k} / 87$

r $/ 63(76)^{\mathrm{a}}$

$\mathbf{r} / 97$

$\mathbf{q} / 97$

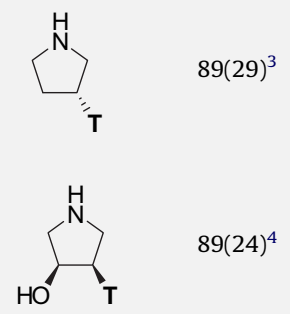

p/96

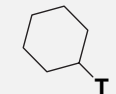

77

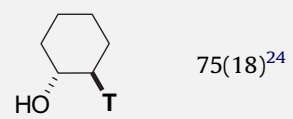

/78

$\mathrm{HO}$
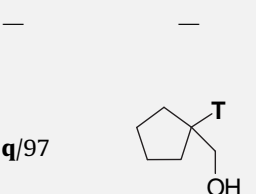

86

$\sim^{\mathrm{OH}}$

$61(45)^{27}$

s/99

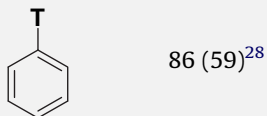

$\mathbf{t} / 90$

$\mathbf{t} / 97$

87

Urea derivatives $\mathbf{9 a - 1}$ and $\mathbf{9 m - t}$ were synthesized using $\mathbf{7 a}$ and $\mathbf{7 b}$, respectively. All cyclizations od $\mathbf{9 - 1 0}$ were carried out with Dower 50 in $\mathrm{H}^{+}$form.

a When Td was used, we obtained $9 \mathbf{r}$ in $76 \%$ yield instead of a $64 \%$ one obtained with $\mathbf{7 b}$.

http://mc.manuscriptcentral.com/poc 
observed the reagent decomposition. We concluded that we needed a reagent more reactive than $\mathbf{6 a}$ and $\mathbf{6 b}$ that would be able to react with the primary amine under milder conditions but which, at the same time, would not be as reactive as the isocyanates $3 \mathbf{a}-\mathbf{b}$. We wanted to avoid the use of these moisturesensitive and extremely reactive agents, which can react with the unprotected hydroxy groups. This property seems to be a main factor responsible for the varying yield of the reported uracil and thymine derivatives (usually $\sim 20-80 \%$, in most cases $<60 \%$ ) found in the literature. ${ }^{11-22}$ We decided, therefore, to modify the original Hřebabecký's reagents $\mathbf{6}^{8}$ by exchanging the ethyl ester group for a more reactive nitrophenyl one.

\section{Results and discussions}

The synthesis of the reagents $\mathbf{7 a}$ and $\mathbf{7 b}$ followed the procedure described for $\mathbf{6},{ }^{8}$ whereby in the last step of this 'one pot' synthesis, a 2-nitrophenol solution in dioxane was added instead of ethanol. The reaction mixture was then concentrated in vacuo and the desired product was obtained by a crystallization from a mixture of toluene and petroleum ether. All procedures were carried out under strict exclusion of moisture under an argon atmosphere. By the same synthetic route we also prepared the 4-nitrophenyl esters 7c but in a lower yield than 7 a (44\% and $57 \%$, resp.) due to a more difficult crystallization of 7c.

The 'uracil reagents' $\mathbf{7 a}$ and $\mathbf{7 c}$ were fully characterized by NMR, MS, and elemental analysis. On the other hand, the characterization of the 'thymine reagent' $\mathbf{7 b}$ appeared to be more difficult due to its unexpectedly high reactivity. The ${ }^{1} \mathrm{H}$ NMR spectrum of $\mathbf{7 b}$ recorded in commercial grade $\mathrm{CDCl}_{3}$ showed a mixture of at least four compounds identified as 3-ethoxy-2-methylacryloylcarbamic acid, 3-ethoxy-2-methylacryloylamide (as the product of decarboxylation of the former compound), 2-nitrophenol, and $\mathbf{7 b}$. If the measurement was performed in $\mathrm{CDCl}_{3}$ adjusted prior to use by passing it through a short pad of anhydrous potassium carbonate, the spectrum did show the appropriate, expected signals of the reagent $\mathbf{7 b}$ but still in the course of the data acquisition $(\sim 1 \mathrm{~h})$, the signals of $\mathbf{7 b}$ gradually disappeared and the signals of 2-nitrophenol and isocyanate $\mathbf{3 b}$ appeared instead. When dry methanol was added to the solution of $\mathbf{7 b}$ in NMR cuvette (in any stage of the NMR measurement), the immediate formation of the methyl ester 11 ( 90\%) was observed. The IR spectrum recorded in potassium carbonate-treated $\mathrm{CDCl}_{3}$ showed the presence of the isocyanate moiety (band at 2244, w). These results clearly showed that the high reactivity of $\mathbf{7 b}$ is caused, in fact, by the presence of isocyanate 3b formed by the spontaneous elimination of 2-nitrophenol from 7b (Scheme 3). In contrast to $\mathbf{7 b}$, the 4-nitrophenyl ester 7d was found to be stable in untreated (commercial grade) $\mathrm{CDCl}_{3}$ so that NMR spectra could be easily recorded.

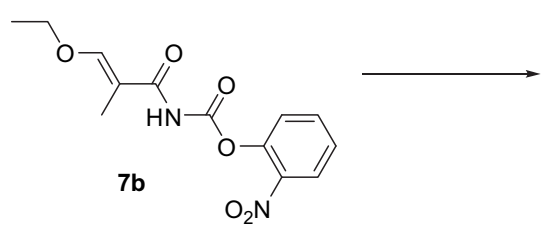

nucleosides, as well as the conformational study, will be published elsewhere. The properties of reagents $\mathbf{7 a}$ and $\mathbf{7 b}$ were further evaluated with a series of commercially available amines $8 \mathbf{d}-\mathbf{m}$. The reactivity of the 4-nitrophenyl 'uracil reagent' 7c was identical with that of 2-nitrophenyl ester 7a (data not shown). The reactions were carried out according to the Scheme 2. The results are summarized in Table 1 (yields given in parentheses refer to the cited literature). In all cases, the cyclization was accomplished by heating the respective urea derivatives 9a-t with Dowex $50\left(\mathrm{H}^{+}\right.$form) in dioxane. Excellent yields of uracil and thymine derivatives were obtained in all cases, as obvious from Table 1.

In the case of free amines, the aminolysis proceeded in dioxane at room temperature. In the case of amine hydrochlorides, the reaction was carried out in DMF with 1 equiv of DBU to release the free amine for the aminolysis. Because of high reactivity of 'thymine reagent' $\mathbf{7 b}$ toward amines bearing free hydroxy groups, the reaction was performed in DMF at $-20^{\circ} \mathrm{C}$ to suppress potentially competing reaction with hydroxyls and thus obtain optimal results. When performing the reaction at room temperature, the formation of side products was observed. A lower yield (63\%) of the urea derivative 9r originating from 3-aminopropane-1,2-diol (8i) seems to be very probably caused by the presence of moisture in the amine $\mathbf{8 i}$. We repeated the synthesis of $\mathbf{1 0 r}$ with the reagent $\mathbf{7 d}$. The aminolysis was carried out in dioxane at rt whereby the urea derivative 9r was obtained in $76 \%$ yield.

Simple amines $\mathbf{8 d}, \mathbf{e}$, as well as the aromatic amines $\mathbf{8 j}$ and $\mathbf{8 m}$, afforded excellent yields in both aminolysis and cyclization reaction. To show the usefulness of the reagents in the case of amines containing free hydroxy groups, the compounds $\mathbf{8 f}-\mathbf{i}$ were selected as a starting material. Amino acid esters 8k,1 were also evaluated, in order to widen the scope of the novel reagent's utility.

\section{Conclusion}

In conclusion, the synthesis of novel, versatile reagents for uracil $(\mathbf{7 a}, \mathbf{7 c})$ and thymine (7b,7d) introduction giving consistently high yields has been developed and its practical usefulness proved. The smooth formation of 1,3-disubstituted urea derivatives and, thus, a broad versatility of the prepared carbamates was proved using a variety of structurally diverse amines containing also free hydroxyl functionalities. The reagents are crystalline solids and can be stored for a long time at rt (7a,7c, and 7d) or in freezer (7b). The use of the 4-nitrophenyl derivatives $\mathbf{7 c}$ and $\mathbf{7 d}$ is highly recommended for the introduction of uracil and thymine moieties, respectively, because of their optimal reactivity and high stability. The use of the 4-nitrophenyl esters of 2-substituted 3-ethoxyacryloylcarbamates is beneficial for the synthesis of 5-substituted uracil derivatives, because high yields are achieved while the method is preparatively simple.

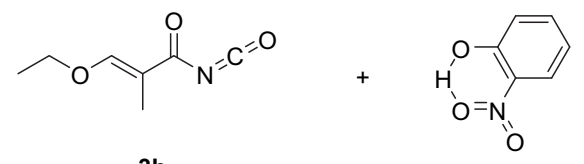

$3 b$

Scheme 3.

The mechanism of the instability of $\mathbf{7 b}$ in the solution is unclear. A detailed study on the reaction kinetics, along with the ab initio calculations, which would explain this phenomenon and especially the differences in reactivity of nitrophenyl esters $\mathbf{7 a - 7 d}$, is underway.

The prepared reagents $\mathbf{7} \mathbf{a}$ and $\mathbf{7 b}$ were used for the synthesis of desired pyrrolidine nucleosides $10 \mathbf{a}-\mathbf{c}$ and $\mathbf{1 0 m}, \mathbf{n}$ in very good yields (see Table 1). A more detailed study on these pyrrolidine

\section{Experimental}

\subsection{General}

Unless stated otherwise, all used solvents were anhydrous. Amines 8d-m were purchased from Sigma-Aldrich (Czech Republic) and used directly. TLC was performed on UV 254 silica gel 
pre-coated aluminum plates (Merck). Compounds were detected by UV light ( $254 \mathrm{~nm}$ ) and/or by spraying with $1 \%$ ethanolic solution of ninhydrin to visualize amines. Preparative column chromatography was carried out on silica gel (40-60 $\mu \mathrm{m}$; Fluka), and the elution was performed at the flow rate of $40 \mathrm{ml} / \mathrm{min}$. The following solvent systems were used for TLC and the preparative chromatography: toluene/ethyl acetate 1:1 (T); chloroform/ethanol 9:1 (C1). The concentrations of solvent systems are stated in volume percents. Analytical RP HPLC was performed on LC5000 Liquid Chromatograph (INGOS-PIKRON, Czech Republic) using Luna C18 (2) column $(4.6 \times 150 \mathrm{~mm})$ at a flow rate of $1 \mathrm{ml} / \mathrm{min}$ under gradient elution of methanol in $0.1 \mathrm{M}$ TEAA pH $7.5(A=0.1 \mathrm{M}$ TEAA; $B=0.1 \mathrm{M}$ TEAA in $50 \%$ aqueous methanol; $C=$ methanol). Mass spectra were recorded on LTQ Orbitrap XL (Thermo Fisher Scientific) instrument using ESI method. Bruker AVANCE $500\left({ }^{1} \mathrm{H}\right.$ at $500 \mathrm{MHz},{ }^{13} \mathrm{C}$ at 125.7 MHz) and Bruker AVANCE $600\left({ }^{1} \mathrm{H}\right.$ at $600.1 \mathrm{MHz},{ }^{13} \mathrm{C}$ at $150.9 \mathrm{MHz}$ ) spectrometers were used for NMR spectra measurement. Chemical shifts (in ppm, $\delta$ scale) were referenced to TMS as internal standard or to a solvent signal; coupling constants $(J)$ are given in $\mathrm{Hz}$. Complete assignment of protons and carbons was done by analysis of correlated homonuclear 2D-COSY and heteronuclear ${ }^{1} \mathrm{H}-{ }^{13} \mathrm{C}$ HSQC and ${ }^{1} \mathrm{H}_{-}{ }^{13} \mathrm{C}$ HMBC spectra. Relative configuration was checked using DPFGSE-NOE and 2D-ROESY techniques. Nucleobase atom numbering was used also in the case of linear intermediates:

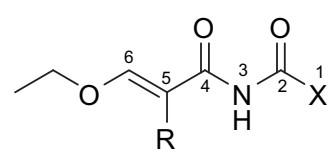

\subsection{General method for aminolysis A}

Reagent 7a, 7c or 7d (1.1 equiv) or $\mathbf{7 b}$ (1.3 equiv) was added to the solution of amine in dioxane $(10 \mathrm{ml} / \mathrm{mmol})$, and the reaction mixture was stirred at rt for $20 \mathrm{~min}$. Solvent was removed in vacuo and the product was obtained by column chromatography on silica gel using a linear gradient of ethyl acetate in toluene.

\subsection{General method for aminolysis B}

Reagent $\mathbf{7 b}$ ( 1.3 equiv) was added to the solution of amine in DMF $(10 \mathrm{ml} / \mathrm{mmol})$ at $-20^{\circ} \mathrm{C}$. The reaction mixture was stirred at $-20^{\circ} \mathrm{C}$ for $20 \mathrm{~min}$. Solvent was removed in vacuo and the product was recovered using column chromatography on silica gel under a linear gradient of ethyl acetate in toluene.

\subsection{General method for aminolysis C}

Reagent 7a (1.1 equiv) or $\mathbf{7 b}$ (1.3 equiv) was added to the solution of amine and DBU ( 1 equiv) in DMF $(10 \mathrm{ml} / \mathrm{mmol})$. The reaction mixture was stirred at $\mathrm{rt}$ for $20 \mathrm{~min}$. Solvent was removed in vacuo and the product was obtained by column chromatography on silica gel using a linear gradient of ethyl acetate in toluene.

\subsection{General method for aminolysis D}

Reagent 7b (1.3 equiv) was added to the solution of amine and DBU (1 equiv) in DMF $(10 \mathrm{ml} / \mathrm{mmol})$ at $-20^{\circ} \mathrm{C}$. The reaction mixture was stirred at $-20^{\circ} \mathrm{C}$ for $20 \mathrm{~min}$. Solvent was removed in vacuo and the product was obtained by column chromatography on silica gel using a linear gradient of ethyl acetate in toluene.

\subsection{General method for cyclization $E$}

Dowex 50 in $\mathrm{H}^{+}$form $(2 \mathrm{~g} / \mathrm{mmol})$ was added to the solution of 9 in dioxane $(10 \mathrm{ml} / \mathrm{mmol})$. The reaction mixture was heated to $90{ }^{\circ} \mathrm{C}$ for $3 \mathrm{~h}$.

4.6.1. 2-Nitrophenyl 3-ethoxyacryloylcarbamate (7a). Ethoxyethylene $(19 \mathrm{ml}, 200 \mathrm{mmol})$ in dioxane $(150 \mathrm{ml})$ was added to a solution of chlorocarbonylisocyanate $(15 \mathrm{~g}, 142 \mathrm{mmol})$ in dioxane $(200 \mathrm{ml})$ at $10^{\circ} \mathrm{C}$ under an argon atmosphere during 20 min followed by triethylamine $(20 \mathrm{ml}, 142 \mathrm{mmol})$ in dioxane $(150 \mathrm{ml})$ at $10^{\circ} \mathrm{C}$ during $20 \mathrm{~min}$ (a white thick precipitate of triethylamine hydrochloride appeared). To this suspension, a solution of 2-nitrophenol (25 g, $180 \mathrm{mmol})$, [co-evaporated with toluene $(2 \times 100 \mathrm{ml})$ and dioxane $(2 \times 100 \mathrm{ml})]$, in dry dioxane $(200 \mathrm{ml})$ was added at $10^{\circ} \mathrm{C}$ under argon atmosphere during $30 \mathrm{~min}$. The reaction mixture was stirred at $10^{\circ} \mathrm{C}$ for $30 \mathrm{~min}$, filtered under argon atmosphere, and the filtrate was concentrated in vacuo. The residue was triturated with $20 \%$ petroleum ether in toluene $(250 \mathrm{ml})$. The yellowish crystals were immediately filtered off (under argon atmosphere), washed with $20 \%$ petroleum ether in toluene $(2 \times 50 \mathrm{ml})$ and then with petroleum ether $(100 \mathrm{ml})$, and finally dried in vacuo over phosphorus pentoxide. The desired reagent 7a was obtained in $57 \%$ yield $(22.6 \mathrm{~g}, 80.65 \mathrm{mmol})$ in the form of yellowish crystals (mp $\left.124.6^{\circ} \mathrm{C}\right), \nu_{\max }\left(\mathrm{CHCl}_{3}\right) 3400(\mathrm{~m}), 3127(\mathrm{w})$, 1808 (w, sh), 1783 (s), 1689 (s), 1621 (s, sh), 1605 (vs), 1534 (vs), 1490 (m), 1450 (s, sh), $1376(\mathrm{~m}), 1350$ (s), $1316(\mathrm{~m}), 1251$ (m), 1169 (vs), 1152 (vs), 1087 (s), 1023 (m), 980 (w, sh), 961 (m), 867 (w), 815 (w), 692 (m), $655(\mathrm{w}), 555(\mathrm{vw}), 508(\mathrm{w}), 448(\mathrm{vw}) . \delta_{\mathrm{H}}\left(500.0 \mathrm{MHz}, \mathrm{CDCl}_{3}\right) 1.34$ $\left(3 \mathrm{H}, \quad \mathrm{t}, \quad J\left(\mathrm{CH}_{3}, \mathrm{CH}_{2}\right)=7.1, \quad \mathrm{CH}_{3}\right), \quad 3.98 \quad\left(2 \mathrm{H}, \quad \mathrm{qd}, \quad J\left(\mathrm{CH}_{2}, \mathrm{CH}_{3}\right)=7.1\right.$, $\left.J\left(\mathrm{CH}_{2}, 6\right)=0.5, \mathrm{OCH}_{2} \mathrm{CH}_{3}\right), 6.36(1 \mathrm{H}, \mathrm{d}, J(5,6)=12.3, \mathrm{H}-5), 7.35(1 \mathrm{H}$, dd, $\left.J\left(6^{\prime}, 5^{\prime}\right)=8.2, J\left(6^{\prime}, 4^{\prime}\right)=1.3, \mathrm{H}-6^{\prime}\right), 7.45\left(1 \mathrm{H}, \mathrm{ddd}, J\left(4^{\prime}, 3^{\prime}\right)=8.2, J\left(4^{\prime}, 5^{\prime}\right)=7.4\right.$, $\left.J\left(4^{\prime}, 6^{\prime}\right)=1.3, \mathrm{H}-4^{\prime}\right), 7.69\left(1 \mathrm{H}, \mathrm{ddd}, J\left(5^{\prime}, 6^{\prime}\right)=8.2, J\left(5^{\prime}, 4^{\prime}\right)=7.4, J\left(5^{\prime}, 3^{\prime}\right)=1.6\right.$, $\left.\mathrm{H}-5^{\prime}\right), 7.85\left(1 \mathrm{H}, \mathrm{dt}, J(6,5)=12.3, J\left(6, \mathrm{CH}_{2}\right)=0.5, \mathrm{H}-6\right), 8.13(1 \mathrm{H}, \mathrm{dd}$, $\left.J\left(3^{\prime}, 4^{\prime}\right)=8.2, J\left(3^{\prime}, 5^{\prime}\right)=1.6, \mathrm{H}-3^{\prime}\right), 8.39\left(1 \mathrm{H}\right.$, br s, H-3). $\delta_{\mathrm{C}}(125.7 \mathrm{MHz}$, $\left.\mathrm{CDCl}_{3}\right)$ 14.24 $\left(\mathrm{CH}_{3}\right), 67.31\left(\mathrm{OCH}_{2} \mathrm{CH}_{3}\right), 96.27(\mathrm{C}-5), 125.37\left(\mathrm{C}-6^{\prime}\right), 125.88$ $\left(\mathrm{C}-3^{\prime}\right), 127.09\left(\mathrm{C}-4^{\prime}\right), 134.87\left(\mathrm{C}-5^{\prime}\right), 141.69\left(\mathrm{C}-2^{\prime}\right), 143.15\left(\mathrm{C}-1^{\prime}\right), 149.51$ (C-2), 165.47 (C-6), 167.28 (C-4). For $\mathrm{C}_{12} \mathrm{H}_{12} \mathrm{~N}_{2} \mathrm{O}_{6}$ (280.23) cald: $51.43 \%$ C, $4.32 \mathrm{H}, 10.00 \% \mathrm{~N}$; found: $51.36 \% \mathrm{C}, 4.33 \% \mathrm{H}, 9.82 \% \mathrm{~N}$. HRMS for $\mathrm{C}_{12} \mathrm{H}_{13} \mathrm{~N}_{2} \mathrm{O}_{6}(\mathrm{M}+\mathrm{H})^{+}$calcd 281.0768, found 281.0763.

4.6.2. 2-Nitrophenyl 3-ethoxy-2-methylacryloylcarbamate (7b). The title compound was prepared from 1-ethoxypropene $(22 \mathrm{ml}$, $200 \mathrm{mmol}$ ) using the same procedure as for compound $7 \mathbf{a}$ in $59 \%$ yield ( $24.66 \mathrm{~g}, 83.8 \mathrm{mmol}$ ) of yellowish crystals. Crystallization of the product $\mathbf{7 b}$ proceeded in the refrigerator overnight ( $\mathrm{mp} 139$ $\left.143{ }^{\circ} \mathrm{C}\right), \nu_{\max }(\mathrm{KBr}) 3293(\mathrm{~s}), 3183(\mathrm{w}), 3104(\mathrm{w}), 3084(\mathrm{w}), 3048(\mathrm{w})$, 1787 (vs), 1762 (s), 1678 (s), 1648 (s), 1621 (m), 1605 (m), 1605 (m), 1591 (m), 1533 (vs), 1524 (vs, sh), 1511 (s, sh), 1478 (s), 1450 (m), 1388 (m), 1369 (m, sh), 1352 (s), 1316 (m, sh), 1305 (s), 1211 (vs), 1184 (vs), 1160 (s), 1147 (s), 1122 (s), $1084(\mathrm{~s}), 1026(\mathrm{~m}), 975(\mathrm{~m}), 959$ (w, sh), $870(\mathrm{w}), 858(\mathrm{w}), 777(\mathrm{~m}, \mathrm{sh}), 761(\mathrm{~m}), 747(\mathrm{~m}), 704(\mathrm{~m}), 688$ $(\mathrm{m}), 629(\mathrm{~m}), 559(\mathrm{w}), 435(\mathrm{w}) . \delta_{\mathrm{H}}\left(600.1 \mathrm{MHz}, \mathrm{CDCl}_{3}\right) 1.33(3 \mathrm{H}, \mathrm{t}$, $\left.J\left(\mathrm{CH}_{3}, \mathrm{CH}_{2}\right)=7.1, \mathrm{CH}_{3}\right), 1.83\left(3 \mathrm{H}, \mathrm{d}, J\left(\mathrm{CH}_{3}, 6\right)=1.2,5-\mathrm{CH}_{3}\right), 4.09(2 \mathrm{H}, \mathrm{q}$, $\left.J\left(\mathrm{CH}_{2}, \mathrm{CH}_{3}\right)=7.1, \mathrm{OCH}_{2} \mathrm{CH}_{3}\right), 7.35\left(1 \mathrm{H}, \mathrm{dd}, J\left(6^{\prime}, 5^{\prime}\right)=8.2, J\left(6^{\prime}, 4^{\prime}\right)=1.3, \mathrm{H}-\right.$ $\left.6^{\prime}\right), 7.42\left(1 \mathrm{H}\right.$, ddd, $\left.J\left(4^{\prime}, 3^{\prime}\right)=8.2, J\left(4^{\prime}, 5^{\prime}\right)=7.4, J\left(4^{\prime}, 6^{\prime}\right)=1.3, \mathrm{H}-4^{\prime}\right), 7.50$ $\left(1 \mathrm{H}, \mathrm{q}, J\left(6, \mathrm{CH}_{3}\right)=1.2, \mathrm{H}-6\right), 7.67\left(1 \mathrm{H}\right.$, ddd, $J\left(5^{\prime}, 6^{\prime}\right)=8.2, J\left(5^{\prime}, 4^{\prime}\right)=7.4$, $\left.J\left(5^{\prime}, 3^{\prime}\right)=1.6, \mathrm{H}-5^{\prime}\right), 8.09\left(1 \mathrm{H}, \mathrm{dd}, J\left(3^{\prime}, 4^{\prime}\right)=8.2, J\left(3^{\prime}, 5^{\prime}\right)=1.6, \mathrm{H}-3^{\prime}\right), 8.17$ $\left(1 \mathrm{H}\right.$, br s, H-3). $\delta_{\mathrm{C}}\left(150.9 \mathrm{MHz}, \mathrm{CDCl}_{3}\right) 9.10\left(5-\mathrm{CH}_{3}\right), 15.32\left(\mathrm{CH}_{3} \mathrm{CH}_{2} \mathrm{O}\right)$, $70.44\left(\mathrm{OCH}_{2} \mathrm{CH}_{3}\right), 107.04(\mathrm{C}-5), 125.49\left(\mathrm{C}-6^{\prime}\right), 125.76\left(\mathrm{C}-3^{\prime}\right), 126.89$ $\left(\mathrm{C}-4^{\prime}\right), 134.85\left(\mathrm{C}-5^{\prime}\right), 141.63\left(\mathrm{C}-2^{\prime}\right), 143.26\left(\mathrm{C}-1^{\prime}\right), 148.23(\mathrm{C}-2), 158.68$ (C-6), 165.99 (C-4). Note: $\mathrm{CDCl}_{3}$ must be made anhydrous by treating with $\mathrm{K}_{2} \mathrm{CO}_{3}$ and the NMR spectra must be measured as fast as possible because of the instability of the reagent!!

4.6.3. 4-Nitrophenyl 3-ethoxyacryloylcarbamate (7c). The title compound was prepared from 1-ethoxyethylene $(19 \mathrm{ml}, 200 \mathrm{mmol})$ 
using the same procedure as for compound $7 \mathrm{a}$ in $44 \%$ yield ( $17.5 \mathrm{~g}$, $62.48 \mathrm{mmol}$ ) of light beige crystals. Crystallization of the product 7c proceeded in the refrigerator overnight $\left(\mathrm{mp} 131.3^{\circ} \mathrm{C}\right), \nu_{\max }(\mathrm{KBr})$ $3316(\mathrm{~m}), 3266(\mathrm{~s}), 3195(\mathrm{~m}), 3116(\mathrm{~m}), 3090(\mathrm{~m}), 3060(\mathrm{~m}), 3029(\mathrm{w})$, 2996 (m), 2956 (w), 2912 (w), 2858 (w), 1802 (vs, sh), 1793 (vs), 1766 (m, sh), 1707 (m), 1691 (s), 1629 (vs, br), 1615 (vs, sh), 1595 (s), 1532 (vs, sh), 1516 (vs, br), 1491 (vs), 1473 (s, sh), 1450 (m), 1402 (m), 1362 (vs), 1354 (vs), 1331 (s), 1302 (m), 1259 (vs), 1207 (vs, sh), 1193 (vs, br), 1177 (s, sh), 1143 (vs, br), 1110 (s), 1107 (s), 1094 (m, sh), 1052 (w), 1021 (s), 978 (vs), 970 (s, sh), 954 (m), 871 (vs), 862 (vs), 749 (s), 719 (s), $678(\mathrm{~s}), 633(\mathrm{w}), 529(\mathrm{w}), 494(\mathrm{~m}) . \delta_{\mathrm{H}}\left(500.0 \mathrm{MHz}, \mathrm{CDCl}_{3}\right) 1.37$ $\left(3 \mathrm{H}, \mathrm{t}, J\left(\mathrm{CH}_{3}, \mathrm{CH}_{2}\right)=7.1, C_{3} \mathrm{CH}_{3} \mathrm{O}\right), 4.02\left(2 \mathrm{H}, \mathrm{qd}, J\left(\mathrm{CH}_{2}, \mathrm{CH}_{3}\right)=7.1\right.$, $\left.J\left(\mathrm{CH}_{2}, 6\right)=0.5, \mathrm{OCH}_{2} \mathrm{CH}_{3}\right), 6.40(1 \mathrm{H}, \mathrm{d}, J(5,6)=12.3, \mathrm{H}-5), 7.37(2 \mathrm{H}, \mathrm{m}$, $\left.\mathrm{H}-2^{\prime}, 6^{\prime}\right), 7.87\left(1 \mathrm{H}, \mathrm{dt}, J(6,5)=12.3, J\left(6, \mathrm{CH}_{2}\right)=0.6, \mathrm{H}-6\right), 8.30(2 \mathrm{H}, \mathrm{m}, \mathrm{H}-$ $\left.3^{\prime}, 5^{\prime}\right), 8.34(1 \mathrm{H}$, br s, $\mathrm{H}-3)$. $\delta_{\mathrm{C}}\left(125.7 \mathrm{MHz}, \mathrm{CDCl}_{3}\right) 14.28\left(\mathrm{CH}_{3} \mathrm{CH}_{2} \mathrm{O}\right)$, $67.51\left(\mathrm{OCH}_{2} \mathrm{CH}_{3}\right), 96.23(\mathrm{C}-5), 122.33\left(\mathrm{C}-2^{\prime}, 6^{\prime}\right), 125.12\left(\mathrm{C}-3^{\prime}, 5^{\prime}\right), 145.50$ $\left(\mathrm{C}-4^{\prime}\right), 149.50$ (C-2), $154.53\left(\mathrm{C}-1^{\prime}\right), 165.67$ (C-6), 167.37 (C-4). HRMS for $\mathrm{C}_{12} \mathrm{H}_{13} \mathrm{~N}_{2} \mathrm{O}_{6}(\mathrm{M}+\mathrm{H})^{+}$calcd 281.0768, found 281.0763.

4.6.4. 4-Nitrophenyl 3-ethoxy-2-methylacryloylcarbamate (7d). The title compound was prepared from 1-ethoxypropene $(7.3 \mathrm{ml}$, $66.36 \mathrm{mmol}$ ) using the same procedure as for compound $7 \mathbf{a}$ in $60 \%$ yield $(8.43 \mathrm{~g}, 28.65 \mathrm{mmol})$ of white crystals. Crystallization of the product $7 \mathrm{c}$ proceeded in the refrigerator overnight $\left(\mathrm{mp} 92.4^{\circ} \mathrm{C}\right)$, $\nu_{\max }(\mathrm{KBr}) 3330(\mathrm{~m}, \mathrm{br}, \mathrm{sh}), 3244(\mathrm{~m}), 3173(\mathrm{~m}), 3117(\mathrm{~m}), 3083(\mathrm{~m})$, 3053 (w), 1824 (w), 1780 (vs), 1770 (vs), 1758 (s), 1677 (s, sh), 1661 (s), 1649 (vs), 1615 (m), 1592 (m), 1526 (vs, br), 1488 (vs), 1449 (m), 1388 (w), 1373(m), 1347 (vs), 1305 (s), 1227 (vs, sh), 1218 (vs), 1185 (vs, br), 1114 (vs), 1093 (m, sh), 863 (m, sh), 857 (s), $764(\mathrm{~m}), 746$ $(\mathrm{m}), 709(\mathrm{~m}), 681(\mathrm{~m}), 610-615(\mathrm{w}, \mathrm{br}), 540(\mathrm{w}), 532(\mathrm{w}), 500(\mathrm{w}) . \delta_{\mathrm{H}}$ $\left(499.8 \mathrm{MHz}, \mathrm{CDCl}_{3}\right) 1.34\left(3 \mathrm{H}, \mathrm{t}, J\left(\mathrm{CH}_{3}, \mathrm{CH}_{2}\right)=7.1, \mathrm{CH}_{3} \mathrm{CH}_{3} \mathrm{O}\right), 1.84(3 \mathrm{H}$, d, $\left.J\left(\mathrm{CH}_{3}, 6\right)=1.2,5-\mathrm{CH}_{3}\right), 4.10\left(2 \mathrm{H}, \mathrm{q}, J\left(\mathrm{CH}_{2}, \mathrm{CH}_{3}\right)=7.1, \mathrm{OCH}_{2} \mathrm{CH}_{3}\right), 7.33$ $\left(2 \mathrm{H}, \mathrm{m}, \mathrm{H}-2^{\prime}, 6^{\prime}\right), 7.53\left(1 \mathrm{H}, \mathrm{q}, J\left(6, \mathrm{CH}_{3}\right)=1.2, \mathrm{H}-6\right), 8.24\left(2 \mathrm{H}, \mathrm{m}, \mathrm{H}-3^{\prime}, 5^{\prime}\right)$, $8.30\left(1 \mathrm{H}\right.$, br s, H-3). $\delta_{\mathrm{C}}\left(125.7 \mathrm{MHz}, \mathrm{CDCl}_{3}\right) 9.00\left(5-\mathrm{CH}_{3}\right), 15.25$ $\left(\mathrm{CH}_{3} \mathrm{CH}_{2} \mathrm{O}\right), 70.54\left(\mathrm{OCH}_{2} \mathrm{CH}_{3}\right), 106.95(\mathrm{C}-5), 122.10\left(\mathrm{C}-2^{\prime}, 6^{\prime}\right), 125.12$ $\left(\mathrm{C}-3^{\prime}, 5^{\prime}\right), 145.25\left(\mathrm{C}-4^{\prime}\right), 148.23(\mathrm{C}-2), 154.55\left(\mathrm{C}-1^{\prime}\right), 158.82(\mathrm{C}-6)$, 166.29 (C-4). For $\mathrm{C}_{13} \mathrm{H}_{14} \mathrm{~N}_{2} \mathrm{O}_{6}$ (294.09) calcd: $53.06 \%$ C, $4.80 \mathrm{H}, 9.52 \%$ $\mathrm{N}$; found: $53.00 \% \mathrm{C}, 4.80 \% \mathrm{H}, 9.43 \% \mathrm{~N}$.

4.6.5. (3R)-3-Amino-1-N-Boc-pyrrolidine (8a). A mixture of (3S)-1$\mathrm{N}$-Boc-3-mesyloxypyrrolidine ${ }^{3}(4.87 \mathrm{~g}, 18.35 \mathrm{mmol})$ and sodium azide $(6.5 \mathrm{~g}, 100 \mathrm{mmol})$ in $\operatorname{DMF}(150 \mathrm{ml})$ was stirred at $110^{\circ} \mathrm{C}$ for $5 \mathrm{~h}$. DMF was removed in vacuo and the crude azido derivative [HRMS for $\mathrm{C}_{9} \mathrm{H}_{16} \mathrm{~N}_{4} \mathrm{O}_{2}(\mathrm{M}+\mathrm{Na})^{+}$calcd 235.1165, found 235.1166] was purified using column chromatography on silica gel employing a linear gradient of ethyl acetate in toluene. The obtained azido derivative was dissolved in ethanol $(200 \mathrm{ml})$ and on addition of Pd/C (0.4 g), it was hydrogenated at $10 \mathrm{psi}$ overnight. Pd catalyst was filtered over Celite. Ethanol was then evaporated giving a $73 \%(2.5 \mathrm{~g}, 13.49 \mathrm{mmol})$ overall yield of title compound in the form of yellowish oil, which needed no further purification (NMR checking). $\nu_{\max }\left(\mathrm{CHCl}_{3}\right) 3379$ (w), 3315 (w), 3165 (w, br), 2980 (vs), 1685 (vs, vbr), 1478 (s), 1455 (s), 1413 (vs, vbr), 1390 (vs, sh), 1367 (vs), 1249 (s), 1168 (vs, br), 1130 (vs, sh), 1123 (vs), $461(\mathrm{w}) . \delta_{\mathrm{H}}\left(500.0 \mathrm{MHz}\right.$, DMSO- $d_{6}$ mixture of two amidic isomers 1:1) $1.38\left(18 \mathrm{H}, \mathrm{s},\left(\mathrm{CH}_{3}\right)_{3} \mathrm{C}\right), 1.51$ and $1.85(2 \times 2 \mathrm{H}, 2 \times \mathrm{br}$ $\mathrm{m}, \mathrm{H}-4), 2.87\left(2 \mathrm{H}, \mathrm{dd}, J_{g e m}=10.6, J(2 \mathrm{~b}, 3)=4.8, \mathrm{H}-2 \mathrm{~b}\right), 3.14-3.22(2 \mathrm{H}$, $\mathrm{m}, \mathrm{H}-5 \mathrm{~b}), 3.26-3.34(4 \mathrm{H}, \mathrm{m}, \mathrm{H}-2 \mathrm{a}, 5 \mathrm{a}), 3.37$ and $3.38(2 \times 2 \mathrm{H}, 2 \times \mathrm{m}, \mathrm{H}-$ 3). $\delta_{\mathrm{C}}\left(125.7 \mathrm{MHz}\right.$, DMSO- $d_{6}$, mixture of two amidic isomers $\left.1: 1\right)$ $28.44\left(\left(\mathrm{CH}_{3}\right)_{3} \mathrm{C}\right), 33.64$ and $34.39(\mathrm{C}-4), 44.26$ and $44.47(\mathrm{C}-5), 50.30$ and $51.18(\mathrm{C}-3), 54.06$ and $54.35(\mathrm{C}-2), 78.20\left(\mathbf{C}\left(\mathrm{CH}_{3}\right)_{3}\right), 153.85$ and $153.90(\mathrm{CO})$. HRMS for $\mathrm{C}_{9} \mathrm{H}_{19} \mathrm{~N}_{2} \mathrm{O}_{2}(\mathrm{M}+\mathrm{H})^{+}$calcd 187.1441, found 187.1439.

4.6.6. (3S,4R)-4-Amino-1-N-Boc-3-dimethoxytrityloxypyrrolidine (8b). A mixture of (3S,4S)-1-N-Boc-4-dimethoxytrityloxy-3-mesyloxypyrrolidine $^{4}(8 \mathrm{~g}, 13.69 \mathrm{mmol})$ and sodium azide $(4.3 \mathrm{~g}$,
$66 \mathrm{mmol})$ in DMF $(130 \mathrm{ml})$ was stirred at $110^{\circ} \mathrm{C}$ for $5 \mathrm{~h}$. DMF was removed in vacuo, the residue was dissolved in ethyl acetate, and the solution filtered through a pad of Celite and finally submitted to flash chromatography on silica gel using linear gradient of ethyl acetate in toluene. The obtained crude azido derivative was dissolved, without further purification in ethanol $(200 \mathrm{ml})$, the $\mathrm{Pd} / \mathrm{C}$ catalyst $(0.4 \mathrm{~g})$ was added, and the whole was hydrogenated at $10 \mathrm{psi}$ for $6 \mathrm{~h}$. The catalyst was then filtered off using Celite, ethanol was evaporated, and the title compound was obtained by column chromatography on silica gel using a linear gradient of ethanol in chloroform in $72 \%$ yield $(4.98 \mathrm{~g}, 9.87 \mathrm{mmol})$ in the form of white foam. $\nu_{\max }(\mathrm{KBr}) 3057(\mathrm{w}), 3036(\mathrm{w}), 3000$ (w), $2972(\mathrm{~m}), 2836(\mathrm{w}), 1695(\mathrm{vs}), 1608(\mathrm{~m}), 1582(\mathrm{w}), 1509(\mathrm{~s}), 1492$ (w, sh), $1477(\mathrm{w}), 1463(\mathrm{~m}), 1456(\mathrm{~m}), 1446(\mathrm{~m}), 1408(\mathrm{~s}), 1391(\mathrm{~m}$, sh), 1365 (m), $1302(\mathrm{~m}), 1252(\mathrm{~s}), 1177$ (s), 1155 (m, sh), 1117 (w), $1088(\mathrm{~s}), 1034(\mathrm{~m}), 1015$ (w, sh), $1000(\mathrm{w}), 912(\mathrm{w}), 829(\mathrm{~m}), 755$ $(\mathrm{w}), 702(\mathrm{w}), 627(\mathrm{w}) . \delta_{\mathrm{H}}\left(500.0 \mathrm{MHz}\right.$, DMSO- $d_{6}$ mixture of two amidic isomers $1: 1) 1.28$ and $1.32\left(2 \times 9 \mathrm{H}, 2 \times \mathrm{s},\left(\mathrm{CH}_{3}\right)_{3} \mathrm{C}\right), 2.44(1 \mathrm{H}$, $\left.\mathrm{dd}, J_{g e m}=10.8, J(2 \mathrm{~b}, 3)=6.6, \mathrm{H}-2 \mathrm{~b}\right), 2.64\left(1 \mathrm{H}, \mathrm{dd}, J_{g e m}=10.8\right.$, $J(2 \mathrm{a}, 3)=7.3, \mathrm{H}-2 \mathrm{a}), 2.71(1 \mathrm{H}, \mathrm{m}, \mathrm{H}-4), 2.72\left(1 \mathrm{H}, \mathrm{dd}, J_{\text {gem }}=10.9\right.$, $J(2 \mathrm{~b}, 3)=7.2, \mathrm{H}-2 \mathrm{~b}), 2.86\left(1 \mathrm{H}, \mathrm{dd}, J_{\text {gem }}=10.8, J(2 \mathrm{a}, 3)=7.8, \mathrm{H}-2 \mathrm{a}\right)$, 2.90-2.99 ( $3 \mathrm{H}, \mathrm{m}, \mathrm{H}-4$ and $2 \times \mathrm{H}-5 \mathrm{~b}), 3.02-3.07(2 \mathrm{H}, \mathrm{m}, 2 \times \mathrm{H}-5 \mathrm{a})$, $3.73\left(12 \mathrm{H}, \quad \mathrm{s}, \quad \mathrm{CH}_{3} \mathrm{O}-\mathrm{DMTr}\right), 3.83(1 \mathrm{H}, \mathrm{ddd}, J(3,2)=7.3,6.6$, $J(3,4)=4.4, \mathrm{H}-3), 3.86(1 \mathrm{H}, \mathrm{ddd}, J(3,2)=7.8,7.2, J(3,4)=4.6, \mathrm{H}-3)$, $6.90\left(8 \mathrm{H}, \mathrm{m}, \mathrm{H}-\mathrm{m}-\mathrm{C}_{6} \mathrm{H}_{4}-\mathrm{DMTr}\right), 7.23\left(2 \mathrm{H}, \mathrm{m}, \mathrm{H}-\mathrm{p}-\mathrm{C}_{6} \mathrm{H}_{5}-\mathrm{DMTr}\right)$, 7.29-7.36 (12H, m, H-o- $\mathrm{C}_{6} \mathrm{H}_{4}$-DMTr and $\mathrm{H}-m-\mathrm{C}_{6} \mathrm{H}_{5}$-DMTr), 7.44 and $7.47\left(2 \times 2 \mathrm{H}, 2 \times \mathrm{m}, \mathrm{H}-0-\mathrm{C}_{6} \mathrm{H}_{5}-\mathrm{DMTr}\right) . \delta_{\mathrm{C}}\left(125.7 \mathrm{MHz}\right.$, DMSO- $d_{6}$, mixture of two amidic isomers 1:1) 28.26 and $28.35\left(\left(\mathrm{CH}_{3}\right) \mathrm{C}\right)$, 47.64 and 47.95 (C-2), 51.32 (C-5), 51.75 (C-4), 51.89 (C-5), 52.04 (C-4), 55.27 and $55.29\left(\mathrm{CH}_{3} \mathrm{O}-\mathrm{DMTr}\right), 73.21$ and $73.72(\mathrm{C}-3), 78.17$ and $78.36\left(\mathbf{C}\left(\mathrm{CH}_{3}\right)_{3}\right), 86.17$ (C-DMTr), $113.57\left(\mathrm{C}-\mathrm{m}-\mathrm{C}_{6} \mathrm{H}_{4}-\mathrm{DMTr}\right)$, 126.96 and 127.04 (C-p- $\mathrm{C}_{6} \mathrm{H}_{5}$-DMTr), 127.79 and 127.86 (C-o$\mathrm{C}_{6} \mathrm{H}_{5}$-DMTr), 128.19 (C-m- $\mathrm{C}_{6} \mathrm{H}_{5}$-DMTr), 129.94, 129.99 and 130.05 (C-o- $\mathrm{C}_{6} \mathrm{H}_{4}$-DMTr), 136.30, 136.51 and $136.64\left(\mathrm{C}-\mathrm{i}-\mathrm{C}_{6} \mathrm{H}_{4}\right.$-DMTr), 145.79 and $145.83\left(\mathrm{C}-i-\mathrm{C}_{6} \mathrm{H}_{5}-\mathrm{DMTr}\right), 153.71$ and $153.82(\mathrm{CO})$, 158.44 and $158.53\left(\mathrm{C}-p-\mathrm{C}_{6} \mathrm{H}_{4}\right.$-DMTr). HRMS for $\mathrm{C}_{30} \mathrm{H}_{36} \mathrm{~N}_{2} \mathrm{O}_{5} \mathrm{Na}$ $(\mathrm{M}+\mathrm{Na})^{+}$calcd 527.2516, found 527.2512.

4.6.7. (3S,4S)-4-Amino-1-N-Boc-3-dimethoxytrityloxypyrrolidine (8c). A mixture of (3R,4S)-1-N-Boc-4-dimethoxytrityloxy-3-mesyloxypyrrolidine $^{4}(4.28 \mathrm{~g}, 7.33 \mathrm{mmol})$ and sodium azide $(2.38 \mathrm{~g}$, $37 \mathrm{mmol})$ in DMF $(80 \mathrm{ml})$ was stirred at $110^{\circ} \mathrm{C}$ for $5 \mathrm{~h}$. DMF was removed in vacuo, and the residue was dissolved in ethyl acetate and filtered through a pad of Celite followed by purification on silica gel using a linear gradient of ethyl acetate in toluene. The obtained crude azido derivative was dissolved in ethanol $(200 \mathrm{ml})$, $\mathrm{Pd} / \mathrm{C}(0.2 \mathrm{~g})$ was added, and the whole was hydrogenated for $6 \mathrm{~h}$. The catalyst was filtered off over Celite, ethanol was evaporated, and the title compound was recovered using column chromatography on silica gel under linear gradient of ethanol in chloroform in a $68 \%$ yield $(2.53 \mathrm{~g}, 5.01 \mathrm{mmol})$ in the form of white foam. $\nu_{\max }(\mathrm{KBr})$ $3382(\mathrm{w}), 3322(\mathrm{w}), 3057(\mathrm{w}), 3036(\mathrm{w}), 3000(\mathrm{w}), 2973(\mathrm{~m}), 2836$ (w), 1736 (m), 1694 (vs), 1608 (s), 1582 (w), 1509 (vs), 1491 (m, sh), 1478 (m), 1463 (m), 1456 (m), 1446 (m), 1408 (vs), 1392 (s, sh), 1365 (m), 1301 (m), 1252 (vs), 1175 (vs), 1155 (s, sh), $1114(\mathrm{~s}), 1034(\mathrm{~s})$, 1009 (m), 1002 (w, sh), 912 (w), 828 (s), 770 (m), 754 (m), 702 (m), $627(\mathrm{w}) . \delta_{\mathrm{H}}\left(500.0 \mathrm{MHz}\right.$, DMSO- $d_{6}$ mixture of two amidic isomers $1: 1) 1.29$ and $1.37\left(2 \times 9 \mathrm{H}, 2 \times \mathrm{s},\left(\mathrm{CH}_{3}\right)_{3} \mathrm{C}\right), 2.39(1 \mathrm{H}, \mathrm{dd}, \mathrm{Jgem}=11.7$, $J(2 \mathrm{~b}, 3)=2.4, \mathrm{H}-2 \mathrm{~b}), 2.65\left(1 \mathrm{H}, \mathrm{dd}, J_{g e m}=11.7, J(2 \mathrm{~b}, 3)=1.9, \mathrm{H}-2 \mathrm{~b}\right), 2.77$ $\left(1 \mathrm{H}, \mathrm{dd}, J_{\text {gem }}=11.7, J(2 \mathrm{a}, 3)=4.8, \mathrm{H}-2 \mathrm{a}\right), 2.87\left(1 \mathrm{H}, \mathrm{dd}, J_{\text {gem }}=10.9\right.$, $J(5 \mathrm{~b}, 4)=3.0, \mathrm{H}-5 \mathrm{~b}), 2.89(1 \mathrm{H}, \mathrm{dt}, J(4,5)=5.0,2.5, J(4,3)=2.5, \mathrm{H}-4)$, $2.92\left(1 \mathrm{H}, \mathrm{dd}, J_{\mathrm{gem}}=10.5, J(5 \mathrm{~b}, 4)=2.5, \mathrm{H}-5 \mathrm{~b}\right), 2.96\left(1 \mathrm{H}, \mathrm{dd}, J_{\text {gem }}=11.7\right.$, $J(2 \mathrm{a}, 3)=4.9, \mathrm{H}-2 \mathrm{a}), 3.01(1 \mathrm{H}, \mathrm{dt}, J(4,5)=5.6,3.0, J(4,3)=3.0, \mathrm{H}-4), 3.37$ $\left(1 \mathrm{H}, \mathrm{dd}, J_{\text {gem }}=10.9, J(5 \mathrm{a}, 4)=5.6, \mathrm{H}-5 \mathrm{a}\right), 3.39\left(1 \mathrm{H}, \mathrm{dd}, J_{\text {gem }}=10.5\right.$, $J(5 \mathrm{a}, 4)=5.0, \mathrm{H}-5 \mathrm{a}), 3.69(1 \mathrm{H}, \mathrm{ddd}, J(3,2)=4.8,2.4, J(3,4)=3.0, \mathrm{H}-3)$, $3.71(1 \mathrm{H}$, ddd, $J(3,2)=4.9,1.9, J(3,4)=2.5, \mathrm{H}-3), 3.730,3.732$ and http://mc.manuscriptcentral.com/poc 
$3.736\left(12 \mathrm{H}, 3 \times \mathrm{s}, \mathrm{CH}_{3} \mathrm{O}-\mathrm{DMTr}\right), 6.90$ (8H, m, H-m- $\left.\mathrm{C}_{6} \mathrm{H}_{4}-\mathrm{DMTr}\right), 7.20-$ $7.33\left(14 \mathrm{H}, \mathrm{m}, \mathrm{H}-\mathrm{o}-\mathrm{C}_{6} \mathrm{H}_{4}-\mathrm{DMTr}\right.$ and $\left.\mathrm{H}-m, p-\mathrm{C}_{6} \mathrm{H}_{5}-\mathrm{DMTr}\right), 7.39$ and $7.42\left(2 \times 2 \mathrm{H}, 2 \times \mathrm{m}, \mathrm{H}-o-\mathrm{C}_{6} \mathrm{H}_{5}-\mathrm{DMTr}\right) . \delta_{\mathrm{C}}\left(125.7 \mathrm{MHz}, \mathrm{DMSO}-d_{6}\right.$, mixture of two amidic isomers $1: 1) 28.27$ and $28.40\left(\left(\mathrm{CH}_{3}\right) \mathrm{C}\right), 50.28$ and $50.49(\mathrm{C}-2), 51.77$ and $52.27(\mathrm{C}-5), 55.25\left(\mathrm{CH}_{3} \mathrm{O}-\mathrm{DMTr}\right), 55.58$ and $56.53(\mathrm{C}-4), 77.98(\mathrm{C}-3), 78.10$ and $78.34\left(\mathbf{C}\left(\mathrm{CH}_{3}\right)_{3}\right), 78.50(\mathrm{C}-3)$, 86.18 and 86.28 (C-DMTr), 113.48 and 113.51 (C-m- $\mathrm{C}_{6} \mathrm{H}_{4}$-DMTr), 126.94 and 127.01 (C-p- $\mathrm{C}_{6} \mathrm{H}_{5}$-DMTr), 127.99 and 128.12 (C-o,m$\mathrm{C}_{6} \mathrm{H}_{5}$-DMTr), 130.15 (C-o- $\mathrm{C}_{6} \mathrm{H}_{4}$-DMTr), 136.32, 136.49 and 136.60 (C$i-\mathrm{C}_{6} \mathrm{H}_{4}$-DMTr), 145.80 (C-i-C $\mathrm{C}_{6} \mathrm{H}_{5}$-DMTr), 153.93 and 153.95 (CO), 158.39, 158.47 and $158.48 \quad\left(\mathrm{C}-p-\mathrm{C}_{6} \mathrm{H}_{4}\right.$-DMTr). HRMS for $\mathrm{C}_{30} \mathrm{H}_{36} \mathrm{~N}_{2} \mathrm{O}_{5} \mathrm{Na}(\mathrm{M}+\mathrm{Na})^{+}$calcd 527.2516, found 527.2512.

4.6.8. 1-((3R)-1-N-Boc-3-Pyrrolidinyl)-3-(3-ethoxyacryloyl)urea $(9 \boldsymbol{a})$. The title compound was prepared according to general method A from compound $8 \mathbf{a}(1.15 \mathrm{~g}, 6.2 \mathrm{mmol})$ using reagent 7a.Yield, 85\% (1.72 g, $5.254 \mathrm{mmol})$ of yellowish foam. $\nu_{\max }\left(\mathrm{CHCl}_{3}\right)$ 3428 (w), 3268 (w, br, sh), 3236 (w), 3132 (w, sh), 3102 (w), 2983 (m), 1700 (s, sh), 1685 (vs, sh), 1676 (vs), 1624 (s), 1611 (s), 1548 (s), 1498 (m, sh), 1487 (m), 1476 (m), 1456 (m), 1412 (s), 1395 (s, sh), $1368(\mathrm{~m}), 1248(\mathrm{~m}), 1167(\mathrm{~s}), 1132(\mathrm{~s}), 977(\mathrm{w}), 960(\mathrm{w}), 846(\mathrm{w}) . \delta_{\mathrm{H}}$ (500.0 MHz, $\mathrm{CDCl}_{3}$, mixture of two amidic isomers 1:1) $1.36(6 \mathrm{H}, \mathrm{t}$, $\left.J\left(\mathrm{CH}_{3}, \mathrm{CH}_{2}\right)=7.1, \mathrm{CH}_{3}\right), 1.46\left(18 \mathrm{H}, \mathrm{s},\left(\mathrm{CH}_{3}\right)_{3} \mathrm{C}\right), 1.89\left(2 \mathrm{H}, \mathrm{br} \mathrm{m}, \mathrm{H}-4^{\prime} \mathrm{b}\right)$, $2.17\left(2 \mathrm{H}\right.$, dddd, $\left.J_{g e m}=13.2, J\left(4^{\prime} \mathrm{a}, 5^{\prime}\right)=7.8,5.9, J\left(4^{\prime} \mathrm{a}, 3^{\prime}\right)=6.3, \mathrm{H}-4^{\prime} \mathrm{a}\right)$, 3.21 and $3.30\left(2 \times 1 \mathrm{H}, 2 \times\right.$ bdd, $\left.J_{g e m}=10.9, J\left(2^{\prime} \mathrm{b}, 3^{\prime}\right)=5.0, \mathrm{H}-2^{\prime} \mathrm{b}\right), 3.39-$ $3.70(4 \mathrm{H}$, br m, H-5') $3.63-3.70(2 \mathrm{H}$, br m, H-2'a), $3.98(4 \mathrm{H}, \mathrm{q}$, $\left.J\left(\mathrm{CH}_{2}, \mathrm{CH}_{3}\right)=7.1, \mathrm{OCH}_{2} \mathrm{CH}_{3}\right), 4.39\left(2 \mathrm{H}, \mathrm{m}, \mathrm{H}-3^{\prime}\right), 5.32(2 \mathrm{H}, \mathrm{d}$, $J(5,6)=12.2, \mathrm{H}-5), 7.63(2 \mathrm{H}, \mathrm{d}, J(6,5)=12.2, \mathrm{H}-6), 8.84(2 \mathrm{H}, \mathrm{br} \mathrm{d}$, $\left.J\left(1,3^{\prime}\right)=7.0, \mathrm{H}-1\right), 9.27$ and $9.40(2 \times 1 \mathrm{H}, 2 \times \mathrm{br} \mathrm{s}, \mathrm{H}-3) . \delta_{\mathrm{C}}(125.7 \mathrm{MHz}$, $\mathrm{CDCl}_{3}$, mixture of two amidic isomers $\left.1: 1\right) 14.50\left(\mathrm{CH}_{3}\right), 28.46$ $\left(\left(\mathrm{CH}_{3}\right)_{3} \mathrm{C}\right), 31.07$ and $31.81\left(\mathrm{C}-4^{\prime}\right), 43.74$ and $44.07\left(\mathrm{C}-5^{\prime}\right), 48.97$ and $49.69\left(\mathrm{C}-3^{\prime}\right), 51.25$ and $51.57\left(\mathrm{C}-2^{\prime}\right), 67.68\left(\mathrm{OCH}_{2} \mathrm{CH}_{3}\right), 79.49$ $\left(\mathbf{C}\left(\mathrm{CH}_{3}\right)_{3}\right), 97.82(\mathrm{C}-5), 154.42$ and $154.82(\mathrm{C}-2$ and $\mathrm{CO}), 162.93$ and 163.04 (C-6), 168.17 (C-4). HRMS for $\mathrm{C}_{15} \mathrm{H}_{25} \mathrm{~N}_{3} \mathrm{O}_{5} \mathrm{Na}(\mathrm{M}+\mathrm{Na})^{+}$calcd 350.1686 , found 350.1687 .

4.6.9. 1-((3S,4R)-1-N-Boc-3-Dimethoxytrityloxy-4-pyrrolidinyl)-3(3-ethoxyacryloyl)urea (9b). The title compound was prepared according to general method A from compound $\mathbf{8 b}(0.95 \mathrm{~g}$, $1.88 \mathrm{mmol}$ ) and reagent $7 \mathrm{a}$ in $93 \%$ yield $(1.13 \mathrm{~g}, 1.13 \mathrm{mmol})$ of yellowish foam. $\nu_{\max }(\mathrm{KBr}) 3261(\mathrm{w}, \mathrm{br}), 3101(\mathrm{w}, \mathrm{br}), 2976(\mathrm{~m})$, 2837 (w), 1701 (vs), 1679 (vs), 1628 (m, sh), 1610 (s), 1577 (w, sh), 1540 (s, br), 1510 (vs), 1492 (m, sh), 1477 (m), 1465 (m, sh), 1446 (m), 1404 (s), 1393 (m, sh), 1366 (m), 1303 (m), 1252 (s), 1222 (m, sh), $1176(\mathrm{~s}), 1176(\mathrm{~s}), 1093(\mathrm{~m}), 1035(\mathrm{~m}), 1017$ (m, sh), $978(\mathrm{w}), 913$ $(\mathrm{vw}), 829(\mathrm{~m}), 771(\mathrm{w}), 701(\mathrm{w}) . \delta_{\mathrm{H}}\left(500.0 \mathrm{MHz}, \mathrm{CDCl}_{3}\right.$, mixture of two amidic isomers $1: 1) 1.36\left(6 \mathrm{H}, \mathrm{t}, J\left(\mathrm{CH}_{3}, \mathrm{CH}_{2}\right)=7.1, \mathrm{CH}_{3}\right), 1.46$ $\left(18 \mathrm{H}, \mathrm{s},\left(\mathrm{CH}_{3}\right)_{3} \mathrm{C}\right), 2.75\left(1 \mathrm{H}, \mathrm{dd}, J_{g e m}=11.6, J\left(2^{\prime} \mathrm{b}, 3^{\prime}\right)=7.2, \mathrm{H}-2^{\prime} \mathrm{b}\right), 2.88$ $\left(1 \mathrm{H}, \mathrm{dd}, J_{\text {gem }}=11.6, J\left(2^{\prime} \mathrm{a}, 3^{\prime}\right)=7.7, \mathrm{H}-2^{\prime} \mathrm{a}\right), 3.16\left(1 \mathrm{H}, \mathrm{dd}, J_{\text {gem }}=11.6\right.$, $\left.J\left(2^{\prime} \mathrm{b}, 3^{\prime}\right)=7.7, \mathrm{H}-2^{\prime} \mathrm{b}\right), 3.22-3.32\left(3 \mathrm{H}, \mathrm{m}, \mathrm{H}-2^{\prime} \mathrm{a}\right.$ and $\left.2 \times 5^{\prime} \mathrm{b}\right), 3.46(1 \mathrm{H}$, br m, H- $\left.4^{\prime}\right), 3.49$ and $3.54\left(2 \times 1 \mathrm{H}, 2 \times \mathrm{dd}, J_{g e m}=11.8, J\left(5^{\prime} \mathrm{a}, 4^{\prime}\right)=2.8, \mathrm{H}-\right.$ $\left.5^{\prime} \mathrm{a}\right), 3.75$ and $3.76\left(2 \times 6 \mathrm{H}, 2 \times \mathrm{s}, \mathrm{CH}_{3} \mathrm{O}-\mathrm{DMTr}\right), 3.80\left(1 \mathrm{H}, \mathrm{br} \mathrm{m}, \mathrm{H}-4^{\prime}\right)$, 3.917 and $3.923\left(2 \times 2 \mathrm{H}, 2 \times \mathrm{q}, J\left(\mathrm{CH}_{2}, \mathrm{CH}_{3}\right)=7.1, \mathrm{OCH}_{2} \mathrm{CH}_{3}\right), 4.12$ and $4.15\left(2 \times 1 \mathrm{H}, 2 \times\right.$ br m, H- $\left.3^{\prime}\right), 5.27$ and $5.31(2 \times 1 \mathrm{H}, 2 \times \mathrm{d}, J(5,6)=12.1$, $\mathrm{H}-5), 6.81$ and $6.83\left(2 \times 4 \mathrm{H}, 2 \times \mathrm{m}, \mathrm{H}-\mathrm{m}_{-} \mathrm{C}_{6} \mathrm{H}_{4}-\mathrm{DMTr}\right), 7.20(2 \mathrm{H}, \mathrm{m}$, $\mathrm{H}-\mathrm{p}-\mathrm{C}_{6} \mathrm{H}_{5}$-DMTr), 7.28 (4H, br m, H-m- $\left.\mathrm{C}_{6} \mathrm{H}_{5}-\mathrm{DMTr}\right), 7.40$ ( $8 \mathrm{H}, \mathrm{br}$ m, $\left.\mathrm{H}-\mathrm{o}-\mathrm{C}_{6} \mathrm{H}_{4}-\mathrm{DMTr}\right), 7.50$ and $7.52\left(2 \times 2 \mathrm{H}, 2 \times \mathrm{m}, \mathrm{H}-\mathrm{o}-\mathrm{C}_{6} \mathrm{H}_{5}-\mathrm{DMTr}\right)$, $7.67(2 \mathrm{H}, \mathrm{d}, J(6,5)=12.1, \mathrm{H}-6), 9.03(1 \mathrm{H}, \mathrm{br} \mathrm{s}, \mathrm{H}-3), 9.28(2 \mathrm{H}, \mathrm{br} \mathrm{d}$, $\left.J\left(1,4^{\prime}\right)=5.6, \mathrm{H}-1\right), 9.31\left(1 \mathrm{H}\right.$, br s, H-3). $\delta_{\mathrm{C}}\left(125.7 \mathrm{MHz}, \mathrm{CDCl}_{3}\right.$, mixture of two amidic isomers 1:1) $14.50\left(\mathrm{CH}_{3}\right), 28.32$ and 28.37 $\left(\left(\mathrm{CH}_{3}\right) \mathrm{C}\right), 48.52$ and $48.81\left(\mathrm{C}-2^{\prime}\right), 49.47$ and $50.34\left(\mathrm{C}-5^{\prime}\right), 51.27$ and $51.74\left(\mathrm{C}-4^{\prime}\right), 55.16\left(\mathrm{CH}_{3} \mathrm{O}-\mathrm{DMTr}\right), 67.42$ and $67.50\left(\mathrm{OCH}_{2} \mathrm{CH}_{3}\right), 71.28$

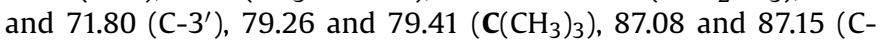
DMTr), 97.92 and 97.99 (C-5), $113.24\left(\mathrm{C}-m-\mathrm{C}_{6} \mathrm{H}_{4}\right.$-DMTr), 126.96 and 127.04 (C-p- $\left.\mathrm{C}_{6} \mathrm{H}_{5}-\mathrm{DMTr}\right), 127.99$ (C-m- $\left.\mathrm{C}_{6} \mathrm{H}_{5}-\mathrm{DMTr}\right), 128.04$ and 128.14 (C-o- $\mathrm{C}_{6} \mathrm{H}_{5}-\mathrm{DMTr}$ ), 129.97 and 130.01 (C-o- $\mathrm{C}_{6} \mathrm{H}_{4}-\mathrm{DMTr}$ ),
136.06, 136.17 and 136.22 (C-i- $\left.\mathrm{C}_{6} \mathrm{H}_{4}-\mathrm{DMTr}\right), 144.87$ and 144.95 (C- $i-\mathrm{C}_{6} \mathrm{H}_{5}$-DMTr), 154.08 and 154.23 (CO), 155.07 and 155.14 (C-2), 158.67 and 158.72 (C-p- $\mathrm{C}_{6} \mathrm{H}_{4}$-DMTr), 162.70 and 162.81 (C-6), 167.50 and $167.80(\mathrm{C}-4)$. HRMS for $\mathrm{C}_{36} \mathrm{H}_{43} \mathrm{~N}_{3} \mathrm{O}_{8} \mathrm{Na}(\mathrm{M}+\mathrm{Na})^{+}$calcd 668.2942 , found 668.2942 .

4.6.10. 1-((3S,4S)-1-N-Boc-3-Dimethoxytrityloxy-4-pyrrolidinyl)-3(3-ethoxyacryloyl)urea (9c). The title compound was prepared according to general method A from compound 8c $(0.9 \mathrm{~g}$, $1.84 \mathrm{mmol})$ and reagent $7 \mathrm{a}$ in $78 \%$ yield $(1.04 \mathrm{~g}, 1.612 \mathrm{mmol})$ of yellowish foam. $\nu_{\max }(\mathrm{KBr}) 3416$ (w, vbr), 3261 (w, br), $3089(\mathrm{w}, \mathrm{br})$, 2976 (m), 2837 (w), 1699 (vs), 1681 (vs), 1628 (m, sh), 1610 (s), 1578 (w, sh), 1549 (s, br), 1509 (s), 1492 (m, sh), 1475 (m), 1466 (m), 1446 (m), 1404 (m, br), 1394 (m, sh), 1367 (m), 1303 (m), 1251 (s), $1174(\mathrm{~s})$, 1111 (m, sh), 1089 (m), 1035 (m), 1018 (w, sh), $976(\mathrm{w}), 913$ (vw), 829 $(\mathrm{m}), 769(\mathrm{w}), 756(\mathrm{w}), 703(\mathrm{w}), \delta_{\mathrm{H}}\left(500.0 \mathrm{MHz}, \mathrm{CDCl}_{3}\right.$, mixture of two amidic isomers $1: 1) 1.33\left(6 \mathrm{H}, \mathrm{t}, J\left(\mathrm{CH}_{3}, \mathrm{CH}_{2}\right)=7.1, \mathrm{CH}_{3}\right), 1.36$ and $1.42\left(2 \times 9 \mathrm{H}, 2 \times \mathrm{s},\left(\mathrm{CH}_{3}\right)_{3} \mathrm{C}\right), 2.48\left(1 \mathrm{H}, \mathrm{dd}, J_{\text {gem }}=12.3, J\left(2^{\prime} \mathrm{b}, 3^{\prime}\right)=3.4, \mathrm{H}-\right.$ $\left.2^{\prime} \mathrm{b}\right), 2.66\left(1 \mathrm{H}, \mathrm{dd}, J_{g e m}=12.3, J\left(2^{\prime} \mathrm{a}, 3^{\prime}\right)=5.3, \mathrm{H}-2^{\prime} \mathrm{a}\right), 2.91(1 \mathrm{H}, \mathrm{dd}$, $\left.J_{\text {gem }}=12.2, \quad J\left(2^{\prime} \mathrm{b}, 3^{\prime}\right)=4.2, \quad \mathrm{H}-2^{\prime} \mathrm{b}\right), \quad 2.99 \quad\left(1 \mathrm{H}, \quad \mathrm{dd}, \quad J_{g e m}=12.2\right.$, $\left.J\left(2^{\prime} \mathrm{a}, 3^{\prime}\right)=5.9, \mathrm{H}-2^{\prime} \mathrm{a}\right), 3.19\left(1 \mathrm{H}, \mathrm{dd}, J_{g e m}=11.7, J\left(5^{\prime} \mathrm{b}, 4^{\prime}\right)=4.8, \mathrm{H}-5^{\prime} \mathrm{b}\right)$, $3.24\left(1 \mathrm{H}, \mathrm{dd}, J_{\text {gem }}=11.4, J\left(5^{\prime} \mathrm{b}, 4^{\prime}\right)=4.5, \mathrm{H}-5^{\prime} \mathrm{b}\right), 3.70\left(1 \mathrm{H}, \mathrm{dd}, J_{\text {gem }}=11.7\right.$, $\left.J\left(5^{\prime} \mathrm{a}, 4^{\prime}\right)=6.1, \mathrm{H}-5^{\prime} \mathrm{a}\right), 3.75$ and $3.76\left(2 \times 6 \mathrm{H}, 2 \times \mathrm{s}, \mathrm{CH}_{3} \mathrm{O}-\mathrm{DMTr}\right), 3.78$ $\left(1 \mathrm{H}, \mathrm{dd}, J_{\text {gem }}=11.4, J\left(5^{\prime} \mathrm{a}, 4^{\prime}\right)=6.4, \mathrm{H}-5^{\prime} \mathrm{a}\right), 3.96\left(4 \mathrm{H}, \mathrm{q}, J\left(\mathrm{CH}_{2}, \mathrm{CH}_{3}\right)=7.1\right.$, $\left.\mathrm{OCH}_{2} \mathrm{CH}_{3}\right), 4.03-4.13\left(2 \mathrm{H}, \mathrm{br} \mathrm{m}, \mathrm{H}-3^{\prime}\right), 4.20$ and $4.36(2 \times 1 \mathrm{H}, 2 \times \mathrm{br} \mathrm{m}$, $\left.\mathrm{H}-4^{\prime}\right), 5.33$ and $5.35(2 \times 1 \mathrm{H}, 2 \times \mathrm{d}, J(5,6)=12.2, \mathrm{H}-5), 6.80$ and 6.82 $\left(2 \times 4 \mathrm{H}, 2 \times\right.$ br m, H-m- $\left.\mathrm{C}_{6} \mathrm{H}_{4}-\mathrm{DMTr}\right), 7.15-7.22\left(2 \mathrm{H}, \mathrm{m}, \mathrm{H}-\mathrm{p}-\mathrm{C}_{6} \mathrm{H}_{5}-\right.$ DMTr), 7.23-7.29 (4H, m, H-m- $\left.\mathrm{C}_{6} \mathrm{H}_{5}-\mathrm{DMTr}\right), 7.31-7.38$ (8H, m, H-o$\mathrm{C}_{6} \mathrm{H}_{4}$-DMTr $), 7.44$ and $7.46\left(2 \times 2 \mathrm{H}, 2 \times \mathrm{m}, \mathrm{H}-\mathrm{o}-\mathrm{C}_{6} \mathrm{H}_{5}-\mathrm{DMTr}\right), 7.63(2 \mathrm{H}$, $\mathrm{d}, J(6,5)=12.1, \mathrm{H}-6), 8.68$ and $8.77\left(2 \times 1 \mathrm{H}, 2 \times\right.$ br d, $\left.J\left(1,4^{\prime}\right)=8.2, \mathrm{H}-1\right)$, 9.33 and $9.47(2 \times 1 \mathrm{H}, 2 \times$ br s, $\mathrm{H}-3) . \delta_{\mathrm{C}}\left(125.7 \mathrm{MHz}, \mathrm{CDCl}_{3}\right.$, mixture of two amidic isomers 1:1) $14.49\left(\mathrm{CH}_{3}\right), 28.30$ and $28.41\left(\left(\mathrm{CH}_{3}\right) \mathrm{C}\right)$, 48.86 and $49.39\left(\mathrm{C}-5^{\prime}\right), 50.13\left(\mathrm{C}-2^{\prime}\right), 54.54\left(\mathrm{C}-4^{\prime}\right), 55.13$ and 55.14 ( $\left.\mathrm{CH}_{3} \mathrm{O}-\mathrm{DMTr}\right), 67.68$ and $67.74\left(\mathrm{OCH}_{2} \mathrm{CH}_{3}\right), 75.40$ and $76.08\left(\mathrm{C}-3^{\prime}\right)$,

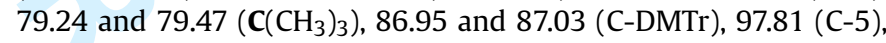
113.24 and 113.28 (C-m- $\left.\mathrm{C}_{6} \mathrm{H}_{4}-\mathrm{DMTr}\right), 126.96$ and $127.01\left(\mathrm{C}-\mathrm{p}-\mathrm{C}_{6} \mathrm{H}_{5}-\right.$ DMTr), 127.92 (C-m- $\left.\mathrm{C}_{6} \mathrm{H}_{5}-\mathrm{DMTr}\right), 128.10$ and $128.24\left(\mathrm{C}-\mathrm{o}-\mathrm{C}_{6} \mathrm{H}_{5}-\right.$ DMTr), 130.10 and 130.13 (C-o- $\left.\mathrm{C}_{6} \mathrm{H}_{4}-\mathrm{DMTr}\right), 136.00,136.17$ and

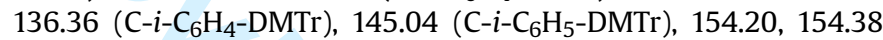
and 154.58 (C-2 and CO), 158.67 and 158.73 (C-p- $\mathrm{C}_{6} \mathrm{H}_{4}-\mathrm{DMTr}$ ), 162.97 (C-6), 167.95 and 168.11 (C-4). HRMS for $\mathrm{C}_{36} \mathrm{H}_{43} \mathrm{~N}_{3} \mathrm{O}_{8} \mathrm{Na}$ $(\mathrm{M}+\mathrm{Na})^{+}$calcd 668.2942, found 668.2941.

4.6.11. 1-Cyclopentyl-3-(3-ethoxyacryloyl)urea (9d). The title compound was prepared according to general method A from compound 8d $(0.2 \mathrm{~g}, 2.348 \mathrm{mmol})$ and reagent $7 \mathrm{a}$ in $88 \%$ yield $(0.47 \mathrm{~g}$, $2.079 \mathrm{mmol}$ ) of white solid. $\nu_{\max }(\mathrm{KBr}) 3422(\mathrm{w}, \mathrm{vbr}), 3258(\mathrm{~m}), 3235$ (m), 3133 (w, sh), 3103 (m), 2985 (m, sh), 2962 (m), 2940 (m, sh), 2869 (w), 1695 (s, sh), 1681 (vs), 1613 (s), 1550 (vs), 1501 (m), 1453 (w), $1473(\mathrm{~m}), 1396(\mathrm{w}), 1367(\mathrm{w}), 1326(\mathrm{w}), 1242(\mathrm{~m}), 1166(\mathrm{~s}), 973$ (w). $\delta_{\mathrm{H}}\left(600.1 \mathrm{MHz}, \mathrm{DMSO}-d_{6}\right) 1.24\left(3 \mathrm{H}, \mathrm{t}, J\left(\mathrm{CH}_{3}, \mathrm{CH}_{2}\right)=7.0, \mathrm{CH}_{3}\right), 1.38$ $\left(2 \mathrm{H}, \mathrm{m}, \mathrm{H}-2^{\prime} \mathrm{a}\right.$ and $\left.\mathrm{H}^{-} 5^{\prime} \mathrm{a}\right), 1.50-1.65\left(4 \mathrm{H}, \mathrm{m}, \mathrm{H}-3^{\prime}\right.$ and $\left.\mathrm{H}-4^{\prime}\right), 1.84(2 \mathrm{H}$, $\mathrm{m}, \mathrm{H}-2^{\prime} \mathrm{b}$ and $\left.\mathrm{H}-5^{\prime} \mathrm{b}\right), 3.93\left(2 \mathrm{H}, \mathrm{q}, J\left(\mathrm{CH}_{2}, \mathrm{CH}_{3}\right)=7.0, \mathrm{OCH}_{2} \mathrm{CH}_{3}\right), 3.98$ $\left(1 \mathrm{H}, \mathrm{m}, \mathrm{H}-1^{\prime}\right), 5.49(1 \mathrm{H}, \mathrm{d}, J(5,6)=12.3, \mathrm{H}-5), 7.54(1 \mathrm{H}, \mathrm{d}, J(6,5)=12.3$, H-6), $8.52\left(1 \mathrm{H}\right.$, br d, $\left.J\left(1,1^{\prime}\right)=7.2, \mathrm{H}-1\right), 10.00\left(1 \mathrm{H}\right.$, br s, H-3). $\delta_{\mathrm{C}}$ (150.9 MHz, DMSO- $\left.d_{6}\right) 14.68\left(\mathrm{CH}_{3}\right), 23.39\left(\mathrm{C}-3^{\prime}\right.$ and $\left.\mathrm{C}-4^{\prime}\right), 32.97$ (C$2^{\prime}$ and $\left.\mathrm{C}-5^{\prime}\right), 50.93\left(\mathrm{C}-1^{\prime}\right), 67.52\left(\mathrm{OCH}_{2} \mathrm{CH}_{3}\right), 98.57$ (C-5), 153.54 (C2), 162.32 (C-6), 168.15 (C-4). HRMS for $\mathrm{C}_{11} \mathrm{H}_{18} \mathrm{~N}_{2} \mathrm{O}_{3} \mathrm{Na}(\mathrm{M}+\mathrm{Na})^{+}$ calcd 249.1210, found 249.1210 .

4.6.12. 1-Cyclohexyl-3-(3-ethoxyacryloyl)urea (9e). The title compound was prepared according to general method A from compound $8 \mathbf{e}(0.233 \mathrm{~g}, 2.348 \mathrm{mmol})$ and reagent $7 \mathbf{a}$ in $88 \%$ yield $(0.5 \mathrm{~g}$, $2.08 \mathrm{mmol}$ ) of white solid. $\nu_{\max }(\mathrm{KBr}) 3382(\mathrm{w}, \mathrm{vbr}), 3256(\mathrm{~m}), 3237$ (m), 3095 (m), $3060(\mathrm{~m}), 2984(\mathrm{~m}), 2977(\mathrm{~m}), 2938(\mathrm{~s}), 2930$ (s),2853 (m), 1698 (s), 1681 (vs), 1613 (vs), 1550 (vs), 1499 (m), 1473 
(m), $1394(\mathrm{w}), 1446(\mathrm{~m}), 1367(\mathrm{w}), 1326(\mathrm{~m}), 1317(\mathrm{~m}), 1252(\mathrm{~m})$, $1242(\mathrm{~s}), 1227(\mathrm{~m}), 1168(\mathrm{vs}), 998(\mathrm{~m}), 955(\mathrm{w}) . \delta_{\mathrm{H}}(500.0 \mathrm{MHz}$, DMSO- $\left.d_{6}\right) 1.24\left(3 \mathrm{H}, \mathrm{t}, \mathrm{J}\left(\mathrm{CH}_{3}, \mathrm{CH}_{2}\right)=7.0, \mathrm{CH}_{3}\right), 1.16-1.35(5 \mathrm{H}, \mathrm{m}, \mathrm{H}-$ $2^{\prime} \mathrm{ax}, 3^{\prime} \mathrm{ax}, 4^{\prime} \mathrm{ax}, 5^{\prime} \mathrm{ax}$ and $\left.\mathrm{H}-6^{\prime} \mathrm{ax}\right), 1.51\left(1 \mathrm{H}, \mathrm{m}, \mathrm{H}-4^{\prime} \mathrm{eq}\right), 1.61(2 \mathrm{H}, \mathrm{m}$, $\mathrm{H}-3^{\prime}$ eq and $\left.\mathrm{H}-5^{\prime} \mathrm{eq}\right), 1.78\left(2 \mathrm{H}, \mathrm{m}, \mathrm{H}-2^{\prime} \mathrm{eq}\right.$ and $\left.\mathrm{H}^{-} 6^{\prime} \mathrm{eq}\right), 3.55(1 \mathrm{H}, \mathrm{m}$, $\left.\mathrm{H}-1^{\prime}\right), 3.94\left(2 \mathrm{H}, \mathrm{q}, J\left(\mathrm{CH}_{2}, \mathrm{CH}_{3}\right)=7.1, \quad \mathrm{OCH}_{2} \mathrm{CH}_{3}\right), 5.49(1 \mathrm{H}, \mathrm{d}$, $J(5,6)=12.3, \mathrm{H}-5), 7.55(1 \mathrm{H}, \mathrm{d}, J(6,5)=12.3, \mathrm{H}-6), 8.52(1 \mathrm{H}, \mathrm{br} \mathrm{d}$, $\left.J\left(1,1^{\prime}\right)=7.6, \mathrm{H}-1\right), 10.00\left(1 \mathrm{H}\right.$, br s, H-3). $\delta_{\mathrm{C}}\left(125.7 \mathrm{MHz}\right.$, DMSO- $\left.d_{6}\right)$ $14.60\left(\mathrm{CH}_{3}\right), 24.25\left(\mathrm{C}-4^{\prime}\right), 25.29\left(\mathrm{C}-3^{\prime}\right.$ and $\left.\mathrm{C}-5^{\prime}\right), 32.58\left(\mathrm{C}-2^{\prime}\right.$ and $\left.\mathrm{C}-6^{\prime}\right), 47.63\left(\mathrm{C}-1^{\prime}\right), 67.45\left(\mathrm{OCH}_{2} \mathrm{CH}_{3}\right), 98.57(\mathrm{C}-5), 153.11(\mathrm{C}-2), 162.20$ (C-6), 168.07 (C-4). HRMS for $\mathrm{C}_{12} \mathrm{H}_{20} \mathrm{~N}_{2} \mathrm{O}_{3} \mathrm{Na}(\mathrm{M}+\mathrm{Na})^{+}$calcd 263.1366, found 263.1366 .

4.6.13. 1-(Ethoxyacryloyl)-3-(trans-2-hydroxycyclohexyl)urea (9f). The title compound was prepared according to general method C from compound $8 \mathbf{8 f}(0.197 \mathrm{~g}, 1.3 \mathrm{mmol})$ and reagent 7a in $99 \%$ yield $(0.33 \mathrm{~g}, 1.287 \mathrm{mmol})$ of white solid. $\nu_{\max }(\mathrm{KBr}) 3512$ (m), 3379 (m, vbr), 3260 (s, vbr), 3135 (m), 3096 (m), 2975 (m), 2936 (s), 2859 (s), 1702 (vs), 1672 (vs, br), 1643 (s, sh), 1620 (vs), 1610 (vs), 1565 (vs, br), 1489 (m), 1474 (m), 1450 (m), 1397 (m), 1372 (w), 1327 (s, sh), 1318 (s), 1252 (s), 1235 (s), 1170 (vs), 1042 (m), $977(\mathrm{~m}) . \delta_{\mathrm{H}}\left(499.8 \mathrm{MHz}\right.$, DMSO- $\left.d_{6}\right) 1.07-1.28\left(4 \mathrm{H}, \mathrm{m}, \mathrm{H}-3^{\prime} \mathrm{ax}\right.$, $4^{\prime} \mathrm{ax}, 5^{\prime} \mathrm{ax}$ and $\left.\mathrm{H}-6^{\prime} \mathrm{ax}\right), 1.24\left(3 \mathrm{H}, \mathrm{t}, J\left(\mathrm{CH}_{3}, \mathrm{CH}_{2}\right)=7.1, \mathrm{CH}_{3}\right), 1.52(1 \mathrm{H}$, m, H-5'eq), 1.60 (1H, m, H-4'eq), 1.80 (1H, m, H- $6^{\prime}$ eq), $1.95(1 \mathrm{H}, \mathrm{m}$, $\left.\mathrm{H}-3^{\prime} \mathrm{eq}\right), 3.23\left(1 \mathrm{H}, \mathrm{m}, \mathrm{H}-1^{\prime}\right), 3.35\left(1 \mathrm{H}, \mathrm{m}, \mathrm{H}-2^{\prime}\right), 3.94(2 \mathrm{H}, \mathrm{q}$, $\left.J\left(\mathrm{CH}_{2}, \mathrm{CH}_{3}\right)=7.1, \mathrm{OCH}_{2} \mathrm{CH}_{3}\right), 4.75\left(1 \mathrm{H}, \mathrm{d}, J\left(\mathrm{OH}, 1^{\prime}\right)=5.4, \mathrm{OH}\right), 5.50$ $(1 \mathrm{H}, \mathrm{d}, J(5,6)=12.4, \mathrm{H}-5), 7.54(1 \mathrm{H}, \mathrm{d}, J(6,5)=12.4, \mathrm{H}-6), 8.56(1 \mathrm{H}$, br d, $\left.J\left(1,2^{\prime}\right)=7.4, \mathrm{H}-1\right), 9.97(1 \mathrm{H}, \mathrm{br} \mathrm{s}, \mathrm{H}-3) . \delta_{\mathrm{C}}(125.7 \mathrm{MHz}$, DMSO$\left.d_{6}\right) 14.58\left(\mathrm{CH}_{3}\right), 23.58\left(\mathrm{C}-5^{\prime}\right), 24.01\left(\mathrm{C}-4^{\prime}\right), 30.99\left(\mathrm{C}-3^{\prime}\right), 34.07(\mathrm{C}-$ $\left.6^{\prime}\right), 54.60\left(\mathrm{C}-2^{\prime}\right), 67.37\left(\mathrm{OCH}_{2} \mathrm{CH}_{3}\right), 71.25\left(\mathrm{C}-1^{\prime}\right), 98.64(\mathrm{C}-5), 153.82$ $(\mathrm{C}-2), 162.01$ (C-6), 167.87 (C-4). HRMS for $\mathrm{C}_{12} \mathrm{H}_{20} \mathrm{~N}_{2} \mathrm{O}_{4} \mathrm{Na}$ $(\mathrm{M}+\mathrm{Na})^{+}$calcd 279.1315 , found 279.1316 .

4.6.14. 1-(3-Ethoxyacryloyl)-3-(cis-2-hydroxycyclopentyl)urea (9g). The title compound was prepared according to general method $\mathrm{C}$ from compound $8 \mathbf{g}(0.179 \mathrm{~g}, 1.3 \mathrm{mmol})$ and reagent $7 \mathbf{a}$ in $95 \%$ yield $(0.30 \mathrm{~g}, 1.238 \mathrm{mmol})$ of white solid. $\nu_{\max }(\mathrm{KBr}) 3435(\mathrm{~s}, \mathrm{br}), 3293$ (m), 3237 (m, br), 3141 (m), 3096 (m), 2983 (m), 2964 (m), 2942 (m), 2872 (w), 1698 (vs), 1676 (vs), 1610 (vs), 1535 (s), 1486 (s), $1474(\mathrm{~m}), 1458(\mathrm{~m}), 1395(\mathrm{w}), 1368$ (w, sh), $1322(\mathrm{~m}), 1246(\mathrm{~m})$, $1185(\mathrm{~s}), 1163(\mathrm{~s}), 1014(\mathrm{~m}), 973(\mathrm{~m}) . \delta_{\mathrm{H}}\left(499.8 \mathrm{MHz}\right.$, DMSO- $\left.d_{6}\right) 1.24$ $\left(3 \mathrm{H}, \mathrm{t}, J\left(\mathrm{CH}_{3}, \mathrm{CH}_{2}\right)=7.0, \mathrm{CH}_{3}\right), 1.40-1.48\left(2 \mathrm{H}, \mathrm{m}, \mathrm{H}-3^{\prime} \mathrm{a}\right.$ and $\left.\mathrm{H}-4^{\prime} \mathrm{a}\right)$, $1.54\left(1 \mathrm{H}, \mathrm{m}, \mathrm{H}-5^{\prime} \mathrm{a}\right), 1.66-1.80\left(2 \mathrm{H}, \mathrm{m}, \mathrm{H}-4^{\prime} \mathrm{b}\right.$ and $\left.\mathrm{H}-5^{\prime} \mathrm{b}\right), 1.84(1 \mathrm{H}$, $\left.\mathrm{m}, \mathrm{H}-3^{\prime} \mathrm{b}\right), 3.80\left(1 \mathrm{H}, \mathrm{m}, \mathrm{H}-2^{\prime}\right), 3.92\left(1 \mathrm{H}, \mathrm{m}, \mathrm{H}-1^{\prime}\right), 3.94(2 \mathrm{H}, \mathrm{q}$, $\left.J\left(\mathrm{CH}_{2}, \mathrm{CH}_{3}\right)=7.0, \mathrm{OCH}_{2} \mathrm{CH}_{3}\right), 4.89\left(1 \mathrm{H}, \mathrm{d}, J\left(\mathrm{OH}, 1^{\prime}\right)=4.5, \mathrm{OH}\right), 5.51(1 \mathrm{H}$, $\mathrm{d}, J(5,6)=12.3, \mathrm{H}-5), 7.53(1 \mathrm{H}, \mathrm{d}, J(6,5)=12.3, \mathrm{H}-6), 8.72(1 \mathrm{H}, \mathrm{br} \mathrm{d}$ $\left.J\left(1,2^{\prime}\right)=7.9, \mathrm{H}-1\right), 9.92(1 \mathrm{H}, \mathrm{br} \mathrm{s}, \mathrm{H}-3) . \delta_{\mathrm{C}}\left(125.7 \mathrm{MHz}\right.$, DMSO- $\left.d_{6}\right)$ $14.59\left(\mathrm{CH}_{3}\right), 20.22\left(\mathrm{C}-4^{\prime}\right), 29.51\left(\mathrm{C}-3^{\prime}\right), 32.73\left(\mathrm{C}-5^{\prime}\right), 54.05\left(\mathrm{C}-2^{\prime}\right)$, $67.33\left(\mathrm{OCH}_{2} \mathrm{CH}_{3}\right), 70.85\left(\mathrm{C}-1^{\prime}\right), 98.69(\mathrm{C}-5), 153.58(\mathrm{C}-2), 161.91(\mathrm{C}-$ 6), 167.56 (C-4). HRMS for $\mathrm{C}_{11} \mathrm{H}_{18} \mathrm{~N}_{2} \mathrm{O}_{4} \mathrm{Na}(\mathrm{M}+\mathrm{Na})^{+}$calcd 265.1159, found 265.1159 .

4.6.15. (RS)-1-(3-(3-Ethoxyacryloyl)ureido)-1-hydroxymethtylcyclopentane $\mathbf{9 h}$. The title compound was prepared according to general method A from compound $\mathbf{8 h}(0.27 \mathrm{~g}, 2.348 \mathrm{mmol})$ and reagent $7 \mathbf{a}$ in $88 \%$ yield $(0.53 \mathrm{~g}, 2.069 \mathrm{mmol})$ of white solid. $\nu_{\max }(\mathrm{KBr}) 3415(\mathrm{~m}$, br), 3273 (m, br, sh), 3220 (m, br), 3127 (m), 3093 (m), $3065(\mathrm{w})$, 2980 (m, sh), 2965 (m), 2879 (w), 1701 (m, sh), 1677 (vs), 1610 (s), 1567 (s, sh), $1556(\mathrm{~s}), 1488(\mathrm{~m}), 1473(\mathrm{~m}), 1448(\mathrm{w}), 1396(\mathrm{w}), 1373$ $(\mathrm{w}), 1328(\mathrm{~m}), 1246(\mathrm{~m}), 1179(\mathrm{~s}), 1156(\mathrm{~s}), 990(\mathrm{w}, \mathrm{sh}) . \delta_{\mathrm{H}}$ $\left(600.1 \mathrm{MHz}, \mathrm{DMSO}-d_{6}\right) 1.24\left(3 \mathrm{H}, \mathrm{t}, J\left(\mathrm{CH}_{3}, \mathrm{CH}_{2}\right)=7.0, \mathrm{CH}_{3}\right), 1.52(2 \mathrm{H}$,

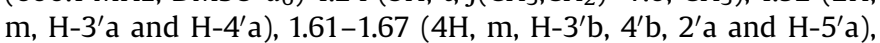
$1.77\left(2 \mathrm{H}, \mathrm{m}, \mathrm{H}-2^{\prime} \mathrm{b}\right.$ and $\left.\mathrm{H}-5^{\prime} \mathrm{b}\right), 3.43\left(2 \mathrm{H}, \mathrm{d}, J\left(\mathrm{CH}_{2}, \mathrm{OH}\right)=5.5, \mathrm{CH}_{2} \mathrm{OH}\right)$, $3.93\left(2 \mathrm{H}, \mathrm{q}, J\left(\mathrm{CH}_{2}, \mathrm{CH}_{3}\right)=7.0, \mathrm{OCH}_{2} \mathrm{CH}_{3}\right), 4.90\left(1 \mathrm{H}, \mathrm{t}, J\left(\mathrm{OH}_{1} \mathrm{CH}_{2}\right)=5.5\right.$, $\mathrm{OH}), 5.50(1 \mathrm{H}, \mathrm{d}, J(5,6)=12.3, \mathrm{H}-5), 7.53(1 \mathrm{H}, \mathrm{d}, J(6,5)=12.3, \mathrm{H}-6)$, $8.64(1 \mathrm{H}, \mathrm{br} \mathrm{s}, \mathrm{H}-1), 9.85(1 \mathrm{H}, \mathrm{br} \mathrm{s}, \mathrm{H}-3) . \delta_{\mathrm{C}}\left(150.9 \mathrm{MHz}, \mathrm{DMSO}-d_{6}\right)$
14.65 $\left(\mathrm{CH}_{3}\right), 24.21\left(\mathrm{C}-3^{\prime}\right.$ and $\left.\mathrm{C}-4^{\prime}\right), 34.39\left(\mathrm{C}-2^{\prime}\right.$ and $\left.\mathrm{C}-5^{\prime}\right), 64.63(\mathrm{C}-$ $\left.1^{\prime}\right), 64.88\left(\mathrm{CH}_{2} \mathrm{OH}\right), 67.42\left(\mathrm{OCH}_{2} \mathrm{CH}_{3}\right), 98.67(\mathrm{C}-5), 153.06(\mathrm{C}-2)$, 162.07 (C-6), 168.01 (C-4). HRMS for $\mathrm{C}_{12} \mathrm{H}_{20} \mathrm{~N}_{2} \mathrm{O}_{4} \mathrm{Na}(\mathrm{M}+\mathrm{Na})^{+}$calcd 279.1315, found 279.1316 .

4.6.16. 1-(2,3-Dihydroxypropyl)-3-(3-ethoxy-2-methylacryloyl)urea $(\mathbf{9 i})$. The title compound was prepared according to general method A from compound $\mathbf{8 i}(0.118 \mathrm{~g}, 1.3 \mathrm{mmol})$ and reagent 7a in $87 \%$ yield $(0.26 \mathrm{~g}, 1.12 \mathrm{mmol})$ of white solid. $\nu_{\max }(\mathrm{KBr}) 3304(\mathrm{~s})$, 3237 (s, br, sh), 3145 (s, br, sh), 2984 (m), 1688 (vs), 1635 (m, sh), 1609 (vs), 1545 (vs), 1500 (m), 1476 (m), 1394 (w), 1365 (m), 1332 (m), $1250(\mathrm{~s}), 1225(\mathrm{~m}), 1162$ (vs), 1095 (s), $1063(\mathrm{~s}), 972(\mathrm{~m}) . \delta_{\mathrm{H}}$ $\left(500.0 \mathrm{MHz}, \mathrm{DMSO}-d_{6}\right) 1.24\left(3 \mathrm{H}, \mathrm{t}, J\left(\mathrm{CH}_{3}, \mathrm{CH}_{2}\right)=7.0, \mathrm{CH}_{3}\right), 3.02(1 \mathrm{H}$, $\left.\mathrm{m}, \mathrm{H}-1^{\prime} \mathrm{a}\right), 3.24\left(1 \mathrm{H}, \mathrm{m}, \mathrm{H}-3^{\prime} \mathrm{a}\right), 3.31-3.38\left(2 \mathrm{H}, \mathrm{m}, \mathrm{H}-1^{\prime} \mathrm{b}\right.$ and $\mathrm{H}-$ $\left.3^{\prime} \mathrm{b}\right), 3.50\left(1 \mathrm{H}, \mathrm{m}, \mathrm{H}-2^{\prime}\right), 3.94\left(2 \mathrm{H}, \mathrm{q}, J\left(\mathrm{CH}_{2}, \mathrm{CH}_{3}\right)=7.0, \mathrm{OCH}_{2} \mathrm{CH}_{3}\right)$, $4.65\left(1 \mathrm{H}, \mathrm{t}, J\left(\mathrm{OH}, 3^{\prime}\right)=5.6,3^{\prime}-\mathrm{OH}\right), 4.90\left(1 \mathrm{H}, \mathrm{d}, J\left(\mathrm{OH}, 2^{\prime}\right)=5.0,2^{\prime}-\mathrm{OH}\right)$, $5.50(1 \mathrm{H}, \mathrm{d}, J(5,6)=12.3, \mathrm{H}-5), 7.55(1 \mathrm{H}, \mathrm{d}, J(6,5)=12.3, \mathrm{H}-6), 8.63$ $\left(1 \mathrm{H}, \mathrm{bt}, J\left(1,1^{\prime}\right)=5.3, \mathrm{H}-1\right), 10.01(1 \mathrm{H}, \mathrm{br} \mathrm{s}, \mathrm{H}-3) . \delta_{\mathrm{C}}(125.7 \mathrm{MHz}$, DMSO-d $\left.d_{6}\right) \quad 14.63\left(\mathrm{CH}_{3}\right), \quad 42.50\left(\mathrm{C}-1^{\prime}\right), \quad 63.88 \quad\left(\mathrm{C}-3^{\prime}\right), \quad 67.44$ $\left(\mathrm{OCH}_{2} \mathrm{CH}_{3}\right), 70.27\left(\mathrm{C}-2^{\prime}\right), 98.63(\mathrm{C}-5), 154.20(\mathrm{C}-2), 162.13(\mathrm{C}-6)$, $167.76(\mathrm{C}-4)$. HRMS for $\mathrm{C}_{9} \mathrm{H}_{16} \mathrm{~N}_{2} \mathrm{O}_{5} \mathrm{Na}(\mathrm{M}+\mathrm{Na})^{+}$calcd 255.0951, found 255.0952 .

4.6.17. 1-(3-Ethoxyacryloyl)-3-phenylurea (9j). The title compound was prepared according to general method A from compound $\mathbf{8 j}$ $(0.091 \mathrm{ml}, 1 \mathrm{mmol})$ and reagent $7 \mathrm{a}$ in $94 \%$ yield $(0.22 \mathrm{~g}, 0.94 \mathrm{mmol})$ of white solid. $\nu_{\max }(\mathrm{KBr}) 3404(\mathrm{w}, \mathrm{br}), 3242(\mathrm{w}), 3230$ (w, br), 3132 (m, br), $3096(\mathrm{~m}), 3063(\mathrm{~m}), 3029(\mathrm{w}), 2980(\mathrm{~m}), 2936(\mathrm{w}), 1710(\mathrm{~s})$, 1682 (vs), 1673 (s, sh), 1621 (vs), 1607 (s, sh), 1597 (vs), 1560 (vs), $1500(\mathrm{~m}), 1495(\mathrm{~m}, \mathrm{sh}), 1472(\mathrm{w}), 1448(\mathrm{~m}), 1393(\mathrm{w}), 1367(\mathrm{vw})$, 1305 (w, sh), 1262 (m), 1245 (s), 1230 (m), 1159 (vs), 1084 (vw), 1018 $(\mathrm{w}), 1002(\mathrm{w}), 987(\mathrm{w}), 847(\mathrm{w}), 758(\mathrm{~m}), 689(\mathrm{w}), 616(\mathrm{vw}), 512(\mathrm{w})$. $\delta_{\mathrm{H}}\left(500.0 \mathrm{MHz}, \mathrm{CDCl}_{3}\right) 1.38\left(3 \mathrm{H}, \mathrm{t}, J\left(\mathrm{CH}_{3}, \mathrm{CH}_{2}\right)=7.1, \mathrm{CH}_{3}\right), 3.98(2 \mathrm{H}, \mathrm{q}$, $\left.J\left(\mathrm{CH}_{2}, \mathrm{CH}_{3}\right)=7.1, \mathrm{OCH}_{2} \mathrm{CH}_{3}\right), 5.43(1 \mathrm{H}, \mathrm{d}, J(5,6)=12.3, \mathrm{H}-5), 7.11(1 \mathrm{H}$, $\mathrm{m}, \mathrm{H}-\mathrm{p}-\mathrm{Ph}), 7.31(2 \mathrm{H}, \mathrm{m}, \mathrm{H}-\mathrm{m}-\mathrm{Ph}), 7.55(2 \mathrm{H}, \mathrm{m}, \mathrm{H}-\mathrm{o}-\mathrm{Ph}), 7.73(1 \mathrm{H}, \mathrm{d}$, $J(6,5)=12.3, \mathrm{H}-6), 9.61$ and $10.85(2 \times 1 \mathrm{H}, 2 \times \mathrm{br} \mathrm{s}, \mathrm{H}-1,3) . \delta_{\mathrm{c}}$ $\left(125.7 \mathrm{MHz}, \mathrm{CDCl}_{3}\right) 14.41\left(\mathrm{CH}_{3}\right), 67.22\left(\mathrm{OCH}_{2} \mathrm{CH}_{3}\right), 97.65(\mathrm{C}-5)$, 120.31 (C-o-Ph), 124.16 (C-p-Ph), 128.87 (C-m-Ph), 137.39 (C-i-Ph), $152.86(\mathrm{C}-2), 163.18(\mathrm{C}-6), 168.39(\mathrm{C}-4)$. HRMS for $\mathrm{C}_{12} \mathrm{H}_{14} \mathrm{~N}_{2} \mathrm{O}_{3} \mathrm{Na}$ $(\mathrm{M}+\mathrm{Na})^{+}$calcd 257.0897, found 257.0894.

4.6.18. Methyl 2-(3-(3-ethoxyacryloyl)ureido)acetate (9k). The title compound was prepared according to general method $C$ from compound $8 \mathbf{k}(0.5 \mathrm{~g}, 3.98 \mathrm{mmol})$ and reagent $7 \mathbf{a}$ in $87 \%$ yield $(0.8 \mathrm{~g}$, $3.475 \mathrm{mmol}$ ) of white solid. $\nu_{\max }(\mathrm{KBr}) 3387$ (w, vbr), 3309 (m, br), $3238(\mathrm{~m}, \mathrm{br}), 3138(\mathrm{~m}, \mathrm{sh}), 3111(\mathrm{~m}), 3095(\mathrm{~m}, \mathrm{sh}), 2984(\mathrm{~m}), 1749$ (vs), 1702 (vs), 1686 (s), 1673 (vs), 1616 (vs), 1570 (vs), 1504 (m), $1476(\mathrm{w}), 1437(\mathrm{~m}), 1401(\mathrm{~m}), 1385(\mathrm{w}), 1180$ (vs), $983(\mathrm{w}) . \delta_{\mathrm{H}}$ $\left(500.0 \mathrm{MHz}, \mathrm{CDCl}_{3}\right) 1.36\left(3 \mathrm{H}, \mathrm{t}, J\left(\mathrm{CH}_{3}, \mathrm{CH}_{2}\right)=7.0, \mathrm{CH}_{3}\right), 3.76(3 \mathrm{H}, \mathrm{s}$, $\left.\mathrm{CH}_{3} \mathrm{O}\right), 3.97\left(2 \mathrm{H}, \mathrm{q}, J\left(\mathrm{CH}_{2}, \mathrm{CH}_{3}\right)=7.0, \mathrm{OCH}_{2} \mathrm{CH}_{3}\right), 4.09(2 \mathrm{H}, \mathrm{d}$, $\left.J\left(\mathrm{CH}_{2}, 1\right)=5.6, \mathrm{CH}_{2}\right), 5.34(1 \mathrm{H}, \mathrm{d}, J(5,6)=12.2, \mathrm{H}-5), 7.66(1 \mathrm{H}, \mathrm{d}$, $J(6,5)=12.2, \mathrm{H}-6), 9.10\left(1 \mathrm{H}, \mathrm{bt}, J\left(1, \mathrm{CH}_{2}\right)=5.6, \mathrm{H}-1\right), 9.66(1 \mathrm{H}, \mathrm{br} \mathrm{s}, \mathrm{H}-$ 3). $\delta_{\mathrm{C}}\left(125.7 \mathrm{MHz}, \mathrm{CDCl}_{3}\right) 14.46\left(\mathrm{CH}_{3}\right), 41.53\left(\mathrm{CH}_{2}\right), 52.28\left(\mathrm{CH}_{3} \mathrm{O}\right)$, $67.49\left(\mathrm{OCH}_{2} \mathrm{CH}_{3}\right), 97.90$ (C-5), 155.51 (C-2), 162.99 (C-6), 168.13 (C4), 169.86 (COO). HRMS for $\mathrm{C}_{9} \mathrm{H}_{14} \mathrm{~N}_{2} \mathrm{O}_{5} \mathrm{Na}(\mathrm{M}+\mathrm{Na})^{+}$calcd 253.0795, found 253.0793 .

4.6.19. Ethyl 2(R)-(3-(3-ethoxyacryloyl)ureido)propanoate (9l). The title compound was prepared according to general method $C$ from compound $81(0.5 \mathrm{~g}, 3.25 \mathrm{mmol})$ and reagent $7 \mathbf{a}$ in $87 \%$ yield $(0.73 \mathrm{~g}, 2.83 \mathrm{mmol})$ of white solid. $\nu_{\max }(\mathrm{KBr}) 3375(\mathrm{~m}, \mathrm{sh}, \mathrm{br})$, 3241 (s, br), 3132 (s), 3107 (s, sh), 2983 (s), 2940 (s), 1742 (vs), 1683 (vs, vbr), 1619 (vs, br), 1560 (vs, sh), 1543 (vs, vbr), 1497 (s), $1473(\mathrm{~s}), 1396$ (s), 1377 (s), 1367 (s), 1211 (vs), 1177 (vs), 1110 (s), $1096(\mathrm{~s}, \mathrm{sh}), 983(\mathrm{~m}), 973(\mathrm{~m}, \mathrm{sh}) . \delta_{\mathrm{H}}\left(500.0 \mathrm{MHz}, \mathrm{DMSO}^{-} d_{6}\right) 1.19$ $\left(3 \mathrm{H}, \mathrm{t}, J\left(\mathrm{CH}_{3}, \mathrm{CH}_{2}\right)=7.1, \mathrm{CH}_{3} \mathrm{CH}_{2} \mathrm{O}-\mathrm{Val}\right), 1.25\left(3 \mathrm{H}, \mathrm{t}, J\left(\mathrm{CH}_{3}, \mathrm{CH}_{2}\right)=7.0\right.$, 
$\left.\mathrm{CH}_{3} \mathrm{CH}_{2} \mathrm{O}\right), 1.33\left(3 \mathrm{H}, \mathrm{d}, J\left(\mathrm{CH}_{3}, \mathrm{CH}\right)=7.2, \mathrm{CH}_{3}-\mathrm{Val}\right), 3.96(2 \mathrm{H}, \mathrm{q}$, $\left.J\left(\mathrm{CH}_{2}, \mathrm{CH}_{3}\right)=7.0, \mathrm{OCH}_{2} \mathrm{CH}_{3}\right), 4.11\left(2 \mathrm{H}, \mathrm{m}, \mathrm{CH}_{3} \mathrm{CH}_{2} \mathrm{O}-\mathrm{Val}\right), 4.30(1 \mathrm{H}$, p, $\left.J\left(\mathrm{CH}_{3} \mathrm{CH}_{3}\right)=J(\mathrm{CH}, 1)=7.2, \mathrm{CH}-\mathrm{Val}\right), 5.51(1 \mathrm{H}, \mathrm{d}, J(5,6)=12.3, \mathrm{H}-5)$, $7.59(1 \mathrm{H}, \mathrm{d}, J(6,5)=12.3, \mathrm{H}-6), 8.87(1 \mathrm{H}, \mathrm{br} \mathrm{d}, J(1, \mathrm{CH})=7.1, \mathrm{H}-1)$, $10.17\left(1 \mathrm{H}\right.$, br s, H-3). $\delta_{\mathrm{C}}\left(125.7 \mathrm{MHz}\right.$, DMSO- $\left.d_{6}\right) 14.23\left(\mathrm{CH}_{3} \mathrm{CH}_{2} \mathrm{O}-\right.$ Val), $14.62\left(\mathrm{CH}_{3} \mathrm{CH}_{2} \mathrm{O}\right), 18.04\left(\mathrm{CH}_{3}-\mathrm{Val}\right), 48.25$ ( $\left.\mathrm{CH}-\mathrm{Val}\right), 60.99$ $\left(\mathrm{CH}_{3} \mathrm{CH}_{2} \mathrm{O}-\mathrm{Val}\right), 67.59\left(\mathrm{OCH}_{2} \mathrm{CH}_{3}\right), 98.40(\mathrm{C}-5), 153.51$ (C-2), 162.57 (C-6), 168.05 (C-4), 172.55 (COO-Val). HRMS for $\mathrm{C}_{11} \mathrm{H}_{18} \mathrm{~N}_{2} \mathrm{O}_{5} \mathrm{Na}$ $(\mathrm{M}+\mathrm{Na})^{+}$calcd 281.1108 , found 281.1108 .

4.6.20. 1-(1-N-Boc-3-pyrrolidinyl)-3-(3-ethoxy-2-methylacryloyl)urea $(9 \mathrm{~m})$. The title compound was prepared according to general method A from compound $8 \mathbf{a}(0.45 \mathrm{~g}, 2.42 \mathrm{mmol})$ and reagent $\mathbf{7 b}$ in $96 \%$ yield $(0.79 \mathrm{~g}, 2.314 \mathrm{mmol})$ of white solid. $\nu_{\max }\left(\mathrm{CHCl}_{3}\right) 3439(\mathrm{w})$, 3270 (m, br), 2983 (m), 1689 (vs, br), 1655 (s, sh), 1614 (s), 1545 (s), 1487 (m, sh), 1476 (s), 1459 (s), 1412 (s), 1392 (s, sh), 1368 (s), 1165 (s), $1137(\mathrm{~s}), 1122(\mathrm{~s}), 462(\mathrm{w}) . \delta_{\mathrm{H}}\left(500.0 \mathrm{MHz}, \mathrm{CDCl}_{3}\right.$, mixture of two amidic isomers 1:1) $1.36\left(6 \mathrm{H}, \mathrm{t}, \mathrm{J}\left(\mathrm{CH}_{3}, \mathrm{CH}_{2}\right)=7.1, \mathrm{OCH}_{2} \mathrm{CH}_{3}\right), 1.46(18 \mathrm{H}$, $\left.\mathrm{s},\left(\mathrm{CH}_{3}\right)_{3} \mathrm{C}\right), 1.78\left(6 \mathrm{H}, \mathrm{d}, \mathrm{J}\left(\mathrm{CH}_{3}, 6\right)=1.2,5-\mathrm{CH}_{3}\right), 1.89\left(2 \mathrm{H}, \mathrm{br} \mathrm{m}, \mathrm{H}-4^{\prime} \mathrm{b}\right)$, $2.16\left(2 \mathrm{H}, \mathrm{dtd}, J_{\text {gem }}=12.8, J\left(4^{\prime} \mathrm{a}, 3^{\prime}\right)=7.4, J\left(4^{\prime} \mathrm{a}, 5^{\prime}\right)=7.4,6.0, \mathrm{H}-4^{\prime} \mathrm{a}\right), 3.21$ and $3.30\left(2 \times 1 \mathrm{H}, 2 \times \mathrm{bdd}, J_{g e m}=11.0, J\left(2^{\prime} \mathrm{b}, 3^{\prime}\right)=4.9, \mathrm{H}-2^{\prime} \mathrm{b}\right), 3.39-3.53$ ( $4 \mathrm{H}$, br m, H-5'), 3.65 (2H, dd, Jgem $\left.=11.0, J\left(2^{\prime} \mathrm{a}, 3^{\prime}\right)=6.4, \mathrm{H}-2^{\prime} \mathrm{a}\right), 4.09$ $\left(4 \mathrm{H}, \mathrm{q}, J\left(\mathrm{CH}_{2}, \mathrm{CH}_{3}\right)=7.1, \mathrm{OCH}_{2} \mathrm{CH}_{3}\right), 4.39\left(2 \mathrm{H}, \mathrm{br} \mathrm{m}, \mathrm{H}-3^{\prime}\right), 7.43(2 \mathrm{H}, \mathrm{q}$, $\left.J\left(6, \mathrm{CH}_{3}\right)=1.2, \mathrm{H}-6\right), 8.17$ and $8.21(2 \times 1 \mathrm{H}, 2 \times$ br s, H-3), $8.93(2 \mathrm{H}$, br d, $\left.J\left(1,3^{\prime}\right)=6.1, \mathrm{H}-1\right) . \delta_{\mathrm{C}}\left(125.7 \mathrm{MHz}, \mathrm{CDCl}_{3}\right.$, mixture of two amidic isomers 1:1) $8.82\left(5-\mathrm{CH}_{3}\right), 15.35\left(\mathrm{OCH}_{2} \mathrm{CH}_{3}\right), 28.44\left(\left(\mathrm{CH}_{3}\right)_{3} \mathrm{C}\right), 31.07$ and $31.89\left(\mathrm{C}-4^{\prime}\right), 43.71$ and $44.08\left(\mathrm{C}-5^{\prime}\right), 48.99$ and $49.74\left(\mathrm{C}-3^{\prime}\right), 51.27$ and $51.62\left(\mathrm{C}-2^{\prime}\right), 70.31\left(\mathrm{OCH}_{2} \mathrm{CH}_{3}\right), 79.46\left(\mathbf{C}\left(\mathrm{CH}_{3}\right)_{3}\right), 106.54$ and $106.64(\mathrm{C}-$ 5), 153.96 and 154.39 (C-2 and $\mathrm{CO}$ ), 157.50 and 157.59 ( $\mathrm{CH}-6), 169.53$ (C-4). HRMS for $\mathrm{C}_{16} \mathrm{H}_{27} \mathrm{~N}_{3} \mathrm{O}_{5} \mathrm{Na}(\mathrm{M}+\mathrm{Na})^{+}$calcd 364.1843, found 364.1842 .

4.6.21. 1-((3S,4R)-1-N-Boc-3-dimethoxytrityloxy-4-pyrrolidinyl)-3(3-ethoxy-2-methylacryloyl)urea (9n). The title compound was prepared according to general method A from compound $\mathbf{8 b}(0.4 \mathrm{~g}$, $0.79 \mathrm{mmol})$ and reagent $\mathbf{7 b}$ in $99 \%$ yield $(0.52 \mathrm{~g}, 0.79 \mathrm{mmol})$ of yellowish foam. $\nu_{\max }(\mathrm{KBr}) 3360$ (w, br, sh), 3255 (m, br), 3130 (w, br), 2976 (m), 2836 (m), 1699 (vs), 1680 (vs, sh), 1663 (s), 1609 (s), 1578 (m), 1538 (s, br), 1510 (vs), 1495 (s, sh), 1476 (s), 1465 (s), 1458 (m, sh), 1446 (m), $1404(\mathrm{~s}), 1392(\mathrm{~s}, \mathrm{sh}), 1366(\mathrm{~m}), 1252(\mathrm{~s}), 1211$ (s), 1176 (s), 1130 (s), 1091 (s), 1034 (s), 1002 (m), 911 (w), 768 (m), 698 (m). $\delta_{\mathrm{H}}\left(500.0 \mathrm{MHz}, \mathrm{CDCl}_{3}\right.$, mixture of two amidic isomers $\left.1: 1\right)$ $1.35\left(6 \mathrm{H}, \mathrm{t}, J\left(\mathrm{CH}_{3}, \mathrm{CH}_{2}\right)=7.1, \mathrm{OCH}_{2} \mathrm{CH}_{3}\right), 1.36$ and $1.38(2 \times 9 \mathrm{H}, 2 \times \mathrm{s}$, $\left.\left(\mathrm{CH}_{3}\right)_{3} \mathrm{C}\right), 1.80$ and $1.82\left(2 \times 3 \mathrm{H}, 2 \times \mathrm{br} \mathrm{s}, 5-\mathrm{CH}_{3}\right), 2.76(1 \mathrm{H}$, dd, $\left.J_{\text {gem }}=11.8, \quad J\left(2^{\prime} \mathrm{b}, 3^{\prime}\right)=7.0, \quad \mathrm{H}-2^{\prime} \mathrm{b}\right), \quad 2.89 \quad\left(1 \mathrm{H}, \quad \mathrm{dd}, \quad J_{g e m}=11.8\right.$, $\left.J\left(2^{\prime} \mathrm{a}, 3^{\prime}\right)=7.7, \mathrm{H}-2^{\prime} \mathrm{a}\right), 3.18\left(1 \mathrm{H}, \mathrm{dd}, J_{g e m}=11.3, J\left(2^{\prime} \mathrm{b}, 3^{\prime}\right)=7.6, \mathrm{H}-2^{\prime} \mathrm{b}\right)$, 3.25 and $3.28\left(2 \times 1 \mathrm{H}, 2 \times \mathrm{dd}, J_{\text {gem }}=11.8, J\left(5^{\prime} \mathrm{b}, 4^{\prime}\right)=5.2, \mathrm{H}-5^{\prime} \mathrm{b}\right), 3.31$ $\left(1 \mathrm{H}, \mathrm{dd}, J_{g e m}=11.3, J\left(2^{\prime} \mathrm{a}, 3^{\prime}\right)=7.2, \mathrm{H}-2^{\prime} \mathrm{a}\right), 3.40\left(1 \mathrm{H}, \mathrm{m}, \mathrm{H}-4^{\prime}\right), 3.49$ and $3.52\left(2 \times 1 \mathrm{H}, 2 \times \mathrm{dd}, J_{\text {gem }}=11.8, J\left(5^{\prime} \mathrm{a}, 4^{\prime}\right)=2.8, \mathrm{H}-5^{\prime} \mathrm{a}\right), 3.777$ and 3.780 $\left(2 \times 6 \mathrm{H}, 2 \times \mathrm{s}, \mathrm{CH}_{3} \mathrm{O}-\mathrm{DMTr}\right), 3.81\left(1 \mathrm{H}, \mathrm{br} \mathrm{m}, \mathrm{H}-4^{\prime}\right), 4.09(4 \mathrm{H}, \mathrm{q}$, $\left.J\left(\mathrm{CH}_{2}, \mathrm{CH}_{3}\right)=7.1, \mathrm{OCH}_{2} \mathrm{CH}_{3}\right), 4.13$ and $4.16\left(2 \times 2 \mathrm{H}, 2 \times \mathrm{br} \mathrm{m}, \mathrm{H}-3^{\prime}\right)$, $6.82\left(8 \mathrm{H}, \mathrm{m}, \mathrm{H}-m-\mathrm{C}_{6} \mathrm{H}_{4}\right.$-DMTr), 7.21 (2H, m, H-p- $\mathrm{C}_{6} \mathrm{H}_{5}$-DMTr), 7.28 ( $4 \mathrm{H}, \mathrm{br} \mathrm{m}, \mathrm{H}-\mathrm{m}-\mathrm{C}_{6} \mathrm{H}_{5}$-DMTr), 7.40 (8H, br m, H-o- $\left.\mathrm{C}_{6} \mathrm{H}_{4}-\mathrm{DMTr}\right), 7.44$ $\left(2 \mathrm{H}, \mathrm{q}, J\left(6, \mathrm{CH}_{3}\right)=1.2, \mathrm{H}-6\right), 7.50$ and $7.51\left(2 \times 2 \mathrm{H}, 2 \times \mathrm{m}, \mathrm{H}-\mathrm{o}-\mathrm{C}_{6} \mathrm{H}_{5}-\right.$ DMTr $), 7.53$ and $7.63(2 \times 1 \mathrm{H}, 2 \times \mathrm{br}$ s, H-3), $9.28(1 \mathrm{H}$, br d, $\left.J\left(1,4^{\prime}\right)=6.4, \mathrm{H}-1\right), 9.30\left(1 \mathrm{H}\right.$, br d, $\left.J\left(1,4^{\prime}\right)=5.6, \mathrm{H}-1\right) . \delta_{\mathrm{C}}(125.7 \mathrm{MHz}$, $\mathrm{CDCl}_{3}$, mixture of two amidic isomers $\left.1: 1\right) 8.94\left(5-\mathrm{CH}_{3}\right), 15.39$ $\left(\mathrm{OCH}_{2} \mathrm{CH}_{3}\right), 28.33$ and $28.39\left(\left(\mathrm{CH}_{3}\right)_{3} \mathrm{C}\right), 48.47$ and $48.88\left(\mathrm{C}-2^{\prime}\right)$, 49.58 and $50.43\left(\mathrm{C}^{-5^{\prime}}\right), 51.29$ and $51.78\left(\mathrm{C}-4^{\prime}\right), 55.19\left(\mathrm{CH}_{3} \mathrm{O}-\mathrm{DMTr}\right)$, $70.33\left(\mathrm{OCH}_{2} \mathrm{CH}_{3}\right), 71.28$ and $71.79\left(\mathrm{C}-3^{\prime}\right), 79.26$ and 79.38 $\left(\mathrm{C}\left(\mathrm{CH}_{3}\right)_{3}\right), 87.05$ and 87.18 (C-DMTr), 106.20 and 106.38 (C-5), 113.25 (C-m- $\mathrm{C}_{6} \mathrm{H}_{4}$-DMTr), 126.96 and 127.04 (C-p- $\mathrm{C}_{6} \mathrm{H}_{5}-\mathrm{DMTr}$ ), 127.92 (C-m- $\mathrm{C}_{6} \mathrm{H}_{5}-\mathrm{DMTr}$ ), 128.06 and 128.13 (C-o- $\mathrm{C}_{6} \mathrm{H}_{5}-\mathrm{DMTr}$ ), 130.00 and 130.05 (C-o- $\mathrm{C}_{6} \mathrm{H}_{4}$-DMTr), 136.02, 136.10 and 136.20 (C$i-\mathrm{C}_{6} \mathrm{H}_{4}$-DMTr), 144.91 and 145.00 (C-i-C $\left.\mathrm{C}_{6} \mathrm{H}_{5}-\mathrm{DMTr}\right), 153.83$ and 153.94 (C-2), 154.12 and 154.27 (CO), 157.44 and 157.53 (C-6),
158.73 (C-p- $\mathrm{C}_{6} \mathrm{H}_{4}-\mathrm{DMTr}$ ), 168.59 and 168.87 (C-4). HRMS for $\mathrm{C}_{37} \mathrm{H}_{45} \mathrm{~N}_{3} \mathrm{O}_{8} \mathrm{Na}(\mathrm{M}+\mathrm{Na})^{+}$calcd 682.3099 , found 682.3096 .

4.6.22. 1-Cyclohexyl-3-(3-Ethoxy-2-methylacryloyl)urea (9o). The title compound was prepared according to general method A from $\mathbf{8 e}$ $(0.128 \mathrm{~g}, 1.3 \mathrm{mmol})$ and reagent $\mathbf{7 b}$ in $82 \%$ yield $(0.27 \mathrm{~g}, 1.062 \mathrm{mmol})$ of white solid. $\nu_{\max }(\mathrm{KBr}) 3352(\mathrm{~m}), 3261(\mathrm{~s}), 3141(\mathrm{~m}), 3089(\mathrm{w}), 2986$ (m), 2934 (s), 2903 (m), 2854 (m), 1678 (vs), 1657 (vs), 1628 (m, sh), 1612 (m, sh), 1547 (vs), $1490(\mathrm{~m}), 1476(\mathrm{~s}), 1466(\mathrm{~s}), 1454(\mathrm{~s}), 1388(\mathrm{~m})$, $1368(\mathrm{~m}), 1295$ (s), 1218 (vs), 1040 (m), $764(\mathrm{~m}) . \delta_{\mathrm{H}}(499.8 \mathrm{MHz}$, $\left.\mathrm{CDCl}_{3}\right) 1.34\left(3 \mathrm{H}, \mathrm{t}, J\left(\mathrm{CH}_{3}, \mathrm{CH}_{2}\right)=7.1, \mathrm{OCH}_{2} \mathrm{CH}_{3}\right), 1.20-1.40(5 \mathrm{H}, \mathrm{m}, \mathrm{H}-$ $\left.2^{\prime}, 3^{\prime}, 4^{\prime}, 5^{\prime}, 6^{\prime} \mathrm{ax}\right), 1.58$ (1H, m, H-4'eq), 1.71 ( $2 \mathrm{H}, \mathrm{m}, \mathrm{H}-3^{\prime}$ eq and $\mathrm{H}_{-} 5^{\prime}$ eq $)$, $1.78\left(3 \mathrm{H}, \mathrm{d}, J\left(\mathrm{CH}_{3}, 6\right)=1.2,5-\mathrm{CH}_{3}\right), 1.92\left(2 \mathrm{H}, \mathrm{m}, \mathrm{H}-2^{\prime}\right.$ eq and $\left.\mathrm{H}-6^{\prime} \mathrm{eq}\right)$, $3.72\left(1 \mathrm{H}, \mathrm{m}, \mathrm{H}-1^{\prime}\right), 4.08\left(2 \mathrm{H}, \mathrm{q}, J\left(\mathrm{CH}_{2}, \mathrm{CH}_{3}\right)=7.1, \mathrm{OCH}_{2} \mathrm{CH}_{3}\right), 7.45(1 \mathrm{H}, \mathrm{q}$, $\left.J\left(6, \mathrm{CH}_{3}\right)=1.2, \mathrm{H}-6\right), 8.33\left(1 \mathrm{H}\right.$, br s, H-3), $8.73\left(1 \mathrm{H}\right.$, br d, $J\left(1,1^{\prime}\right)=7.8, \mathrm{H}-$ 1). $\delta_{\mathrm{C}}\left(125.7 \mathrm{MHz}, \mathrm{CDCl}_{3}\right) 8.85\left(5-\mathrm{CH}_{3}\right), 15.38\left(\mathrm{OCH}_{2} \mathrm{CH}_{3}\right), 24.54\left(\mathrm{C}-3^{\prime}\right.$ and $\left.\mathrm{C}-5^{\prime}\right), 25.51\left(\mathrm{C}-4^{\prime}\right), 32.88\left(\mathrm{C}-2^{\prime}\right.$ and $\left.\mathrm{C}-6^{\prime}\right), 48.40\left(\mathrm{C}-1^{\prime}\right), 70.09$ $\left(\mathrm{OCH}_{2} \mathrm{CH}_{3}\right), 107.04$ (C-5), 153.52 (C-2), 157.05 (C-6), 169.64 (C-4). HRMS $\mathrm{C}_{13} \mathrm{H}_{23} \mathrm{~N}_{2} \mathrm{O}_{3}(\mathrm{M}+\mathrm{H})^{+}$calcd 255.1703, found 255.1704.

4.6.23. 1-(3-Ethoxy-2-methylacryloyl)-3-(trans-2-hydroxycyclohexyl)urea $(\mathbf{9 p})$. The title compound was prepared according to general method D from compound $\mathbf{8 f}(0.128 \mathrm{~g}, 1.3 \mathrm{mmol})$ and reagent $\mathbf{7 b}$ in $96 \%$ yield $(0.26 \mathrm{~g}, 0.962 \mathrm{mmol})$ of white solid. $\nu_{\max }(\mathrm{KBr}) 3432(\mathrm{~s})$, 3264 (s), 3161 (m), 2983 (w), 2940 (m), 2929 (m), 2904 (m), 2861 (m), 1695 (vs), 1687 (vs, sh), 1666 (s), 1607 (m), 1590 (m), 1543 (s), 1532 (s, sh), 1477 (s), 1467 (m, sh), 1451 (m), 1396 (w), 1388 (w, sh), 1370 (w), 1302 (w, sh), $1292(\mathrm{w}), 1215$ (s), $1039(\mathrm{~m}), 766(\mathrm{~m}) . \delta_{\mathrm{H}}$ (499.8 MHz, DMSO-d $\left.d_{6}\right) 1.08-1.26\left(4 \mathrm{H}, \mathrm{m}, \mathrm{H}-3^{\prime} \mathrm{ax}, 4^{\prime} \mathrm{ax}, 5^{\prime} \mathrm{ax}\right.$ and $\mathrm{H}-$ $\left.6^{\prime} \mathrm{ax}\right), 1.25\left(3 \mathrm{H}, \mathrm{t}, \mathrm{J}\left(\mathrm{CH}_{3}, \mathrm{CH}_{2}\right)=7.1, \mathrm{CH}_{2} \mathrm{CH}_{3}\right), 1.53\left(1 \mathrm{H}, \mathrm{m}, \mathrm{H}-5^{\prime} \mathrm{eq}\right), 1.61$ $\left(1 \mathrm{H}, \mathrm{m}, \mathrm{H}-4^{\prime} \mathrm{eq}\right), 1.62\left(3 \mathrm{H}, \mathrm{d}, J\left(\mathrm{CH}_{3}, 6\right)=1.2,5-\mathrm{CH}_{3}\right), 1.80(1 \mathrm{H}, \mathrm{m}, \mathrm{H}-$ $6^{\prime}$ eq), $1.96\left(1 \mathrm{H}, \mathrm{m}, \mathrm{H}-3^{\prime}\right.$ eq $), 3.24\left(1 \mathrm{H}, \mathrm{m}, \mathrm{H}-1^{\prime}\right), 3.38\left(1 \mathrm{H}, \mathrm{m}, \mathrm{H}-2^{\prime}\right)$, $4.05\left(2 \mathrm{H}, \mathrm{q}, J\left(\mathrm{CH}_{2}, \mathrm{CH}_{3}\right)=7.1, \mathrm{OCH}_{2} \mathrm{CH}_{3}\right), 4.75\left(1 \mathrm{H}, \mathrm{d}, J\left(\mathrm{OH}, 1^{\prime}\right)=5.4\right.$, $\mathrm{OH}), 7.53\left(1 \mathrm{H}, \mathrm{q}, J\left(6, \mathrm{CH}_{3}\right)=1.2, \mathrm{H}-6\right), 8.69\left(1 \mathrm{H}, \mathrm{br} \mathrm{d}, J\left(1,2^{\prime}\right)=7.3, \mathrm{H}-1\right)$, $9.64\left(1 \mathrm{H}\right.$, br s, H-3). $\delta_{\mathrm{C}}\left(125.7 \mathrm{MHz}\right.$, DMSO- $\left.d_{6}\right) 9.12\left(5-\mathrm{CH}_{3}\right), 15.46$

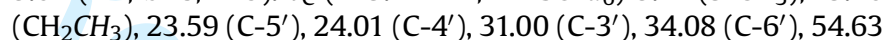
(C-2'), $69.49\left(\mathrm{OCH}_{2} \mathrm{CH}_{3}\right), 71.24\left(\mathrm{C}-1^{\prime}\right), 107.10$ (C-5), 153.88 (C-2), 156.71 (C-6), $169.83(\mathrm{C}-4)$. HRMS $\mathrm{C}_{13} \mathrm{H}_{22} \mathrm{~N}_{2} \mathrm{O}_{4} \mathrm{Na}(\mathrm{M}+\mathrm{Na})^{+}$calcd 293.1472, found 293.1473.

4.6.24. 1-(3-Ethoxy-2-methylacryloyl)-3-(1-hydroxymethylcyclopentyl)urea $(\mathbf{9 q})$. The title compound was prepared according to general method B from compound $\mathbf{8 h}(0.115 \mathrm{~g}, 1.0 \mathrm{mmol})$ and reagent $\mathbf{7 b}$ in $89 \%$ yield $(0.24 \mathrm{~g}, 0.888 \mathrm{mmol})$ of white solid. $\nu_{\max }(\mathrm{KBr}) 3282(\mathrm{~s}, \mathrm{sh}), 3255(\mathrm{~s}), 3210(\mathrm{~s}, \mathrm{br}, \mathrm{sh}), 3112(\mathrm{~m}), 3085(\mathrm{~m}$, sh), 2959 (s), 2930 (m), 2870 (m), 1685 (vs), 1655 (vs), 1612 (m, sh), 1556 (vs), 1492 (m), 1477 (m), 1461 (m), 1452 (m), 1389 (m), 1367 (m), $1312(\mathrm{~m}, \mathrm{sh}), 1300(\mathrm{~s}), 1226(\mathrm{~s}), 1208(\mathrm{~s}), 1051$ (m), $1034(\mathrm{~m}), 767$ (m). $\delta_{\mathrm{H}}\left(500.0 \mathrm{MHz}, \mathrm{CDCl}_{3}\right) 1.33\left(3 \mathrm{H}, \mathrm{t}, \mathrm{J}\left(\mathrm{CH}_{3}, \mathrm{CH}_{2}\right)=7.1, \mathrm{CH}_{2} \mathrm{CH}_{3}\right), 1.68$ ( $2 \mathrm{H}, \mathrm{m}, \mathrm{H}-3^{\prime} \mathrm{a}$ and $\left.\mathrm{H}-4^{\prime} \mathrm{a}\right), 1.77\left(2 \mathrm{H}, \mathrm{m}, \mathrm{H}-3^{\prime} \mathrm{b}\right.$ and $\left.\mathrm{H}-4^{\prime} \mathrm{b}\right) 1.79(3 \mathrm{H}, \mathrm{d}$, $\left.J\left(\mathrm{CH}_{3}, 6\right)=1.2,5-\mathrm{CH}_{3}\right), 1.83-1.87\left(4 \mathrm{H}, \mathrm{m}, \mathrm{H}-2^{\prime}\right.$ and $\left.\mathrm{H}^{-} 5^{\prime}\right), 3.71(2 \mathrm{H}$, br s, $\left.\mathrm{CH}_{2} \mathrm{OH}\right), 4.09\left(2 \mathrm{H}, \mathrm{q}, \mathrm{J}\left(\mathrm{CH}_{2}, \mathrm{CH}_{3}\right)=7.1, \mathrm{OCH}_{2} \mathrm{CH}_{3}\right), 4.28(1 \mathrm{H}$, br s, $\mathrm{OH})$, $7.40\left(1 \mathrm{H}, \mathrm{q}, J\left(6, \mathrm{CH}_{3}\right)=1.2, \mathrm{H}-6\right), 8.10(1 \mathrm{H}, \mathrm{br} \mathrm{s}, \mathrm{H}-1), 9.11(1 \mathrm{H}, \mathrm{br} \mathrm{s}, \mathrm{H}-$ 3). $\delta_{\mathrm{C}}\left(125.7 \mathrm{MHz}, \mathrm{CDCl}_{3}\right) 8.80\left(5-\mathrm{CH}_{3}\right), 15.31\left(\mathrm{CH}_{2} \mathrm{CH}_{3}\right), 23.74\left(\mathrm{C}-3^{\prime}\right.$ and $\left.\mathrm{C}-4^{\prime}\right), 35.69\left(\mathrm{C}-2^{\prime}\right.$ and $\left.\mathrm{C}-5^{\prime}\right), 66.27\left(\mathrm{C}-1^{\prime}\right), 69.13\left(\mathrm{CH}_{2} \mathrm{OH}\right), 70.34$ $\left(\mathrm{OCH}_{2} \mathrm{CH}_{3}\right.$ ), 106.57 (C-5), 154.80 (C-2), 157.58 (C-6), 169.70 (C-4). HRMS $\mathrm{C}_{13} \mathrm{H}_{22} \mathrm{~N}_{2} \mathrm{O}_{4} \mathrm{Na}(\mathrm{M}+\mathrm{Na})^{+}$calcd 293.1472, found 293.1472 .

4.6.25. 1-(2,3-Dihydroxypropyl)-3-(3-ethoxy-2-methylacryloyl)urea (9r). A) The title compound was prepared according to general method B from compound $\mathbf{8 i}(0.118 \mathrm{~g}, 1.3 \mathrm{mmol})$ and reagent $\mathbf{7 b}$ in $63 \%$ yield $(0.20 \mathrm{~g}, 0.813 \mathrm{mmol})$ of white solid. B) The title compound was prepared according to general method A from compound $8 \mathbf{i}(0.177 \mathrm{~g}, 1.95 \mathrm{mmol})$ and reagent $7 \mathbf{d}$ in $76 \%$ yield $(0.38 \mathrm{~g}$, $1.54 \mathrm{mmol}) . \nu_{\max }(\mathrm{KBr}) 3405$ (m, vbr), 3259 (m, br), 3160 (m, br, sh), 2986 (w), 2937 (w), 2893 (w), 1700 (s, sh), 1687 (vs), 1663 (s), 1638 $2986(\mathrm{w}), 2937(\mathrm{w}), 289$ 
(m), 1623 (m, sh), 1591 (m), 1559 (s), 1542 (m, sh), 1506 (w), 1477 (m), 1388 (w, sh), 1368 (w), 1300 (m), 1235 (m, sh), 1212 (s), 1102 (m), $1066(\mathrm{~m}), 1035(\mathrm{~m}), 761(\mathrm{~m}) . \delta_{\mathrm{H}}\left(499.8 \mathrm{MHz}\right.$, DMSO- $\left.d_{6}\right) 1.25$ (3H, t, $\left.J\left(\mathrm{CH}_{3}, \mathrm{CH}_{2}\right)=7.1, \mathrm{CH}_{2} \mathrm{CH}_{3}\right), 1.63\left(3 \mathrm{H}, \mathrm{d}, \mathrm{J}\left(\mathrm{CH}_{3}, 6\right)=1.2,5-\mathrm{CH}_{3}\right)$, $3.04\left(1 \mathrm{H}\right.$, ddd, $\left.J_{\text {gem }}=13.3, J\left(1^{\prime} \mathrm{a}, 2^{\prime}\right)=7.1, J\left(1^{\prime} \mathrm{a}, 1\right)=4.9, \mathrm{H}-1^{\prime} \mathrm{a}\right), 3.25(1 \mathrm{H}$, $\left.\mathrm{dt}, J_{\text {gem }}=10.9, J\left(3^{\prime} \mathrm{a}, \mathrm{OH}\right)=J\left(3^{\prime} \mathrm{a}, 2^{\prime}\right)=6.2, \mathrm{H}-3^{\prime} \mathrm{a}\right), 3.31-3.40(2 \mathrm{H}, \mathrm{m}, \mathrm{H}-$ $1^{\prime} \mathrm{b}$ and $\left.\mathrm{H}^{\prime} 3^{\prime} \mathrm{b}\right), 3.51\left(1 \mathrm{H}, \mathrm{m}, \mathrm{H}-2^{\prime}\right), 4.05\left(2 \mathrm{H}, \mathrm{q}, J\left(\mathrm{CH}_{2}, \mathrm{CH}_{3}\right)=7.1\right.$, $\left.\mathrm{OCH}_{2} \mathrm{CH}_{3}\right), 4.61\left(1 \mathrm{H}, \quad \mathrm{t}, \quad J\left(\mathrm{OH}, 3^{\prime}\right)=5.7, \quad 3^{\prime}-\mathrm{OH}\right), 4.87(1 \mathrm{H}, \mathrm{d}$, $\left.J\left(\mathrm{OH}, 2^{\prime}\right)=5.1,2^{\prime}-\mathrm{OH}\right), 7.53\left(1 \mathrm{H}, \mathrm{q}, J\left(6, \mathrm{CH}_{3}\right)=1.2, \mathrm{H}-6\right), 8.75(1 \mathrm{H}, \mathrm{bdd}$, $\left.J\left(1,1^{\prime} \mathrm{b}\right)=6.0, J\left(1,1^{\prime} \mathrm{a}\right)=5.2, \mathrm{H}-1\right), 9.67(1 \mathrm{H}, \mathrm{br} \mathrm{s}, \mathrm{H}-3) . \delta_{\mathrm{C}}(125.7 \mathrm{MHz}$, DMSO-d $\left.d_{6}\right) 9.16\left(5-\mathrm{CH}_{3}\right), 15.49\left(\mathrm{CH}_{2} \mathrm{CH}_{3}\right), 42.56\left(\mathrm{C}-1^{\prime}\right), 63.88\left(\mathrm{C}-3^{\prime}\right)$, $69.53\left(\mathrm{OCH}_{2} \mathrm{CH}_{3}\right), 70.27\left(\mathrm{C}-2^{\prime}\right), 107.11(\mathrm{C}-5), 154.29(\mathrm{C}-2), 156.77(\mathrm{C}-$ 6), $169.69(\mathrm{C}-4)$. HRMS for $\mathrm{C}_{10} \mathrm{H}_{18} \mathrm{~N}_{2} \mathrm{O}_{5} \mathrm{Na}(\mathrm{M}+\mathrm{Na})^{+}$calcd 269.1108, found 269.1107 .

4.6.26. 1-(3-Ethoxy-2-methylacryloyl)-3-phenylurea (9s). The title compound was prepared according to general method A from compound $8 \mathbf{j}(0.118 \mathrm{~g}, 1.3 \mathrm{mmol})$ and reagent $7 \mathbf{b}$ in $87 \%$ yield $(0.28 \mathrm{~g}, 1.129 \mathrm{mmol})$ of white solid. $\nu_{\max }(\mathrm{KBr}) 3284(\mathrm{~m}), 3197(\mathrm{w})$, 3142 (w), 3088 (w), 3059 (w), 2986 (w), 2937 (w), 2887 (w), 1702 (vs), 1676 (s), 1617 (m), 1605 (s, sh), 1592 (vs), 1557 (s), 1541 (s, sh), 1501 (m), 1488 (m, sh), 1475 (m), 1455 (s), 1396 (m), 1388 (m, sh), 1312 (w), 1226 (s, sh), 1210 (vs), 1179 (w), 1155 (w, sh), 1037 (m), 1002 (w, sh), $916(\mathrm{~m}), 848$ (vw), 757 (s), 690 (m), 618 (vw), $513(\mathrm{w})$. $\delta_{\mathrm{H}}\left(499.8 \mathrm{MHz}, \mathrm{CDCl}_{3}\right) 1.29\left(3 \mathrm{H}, \mathrm{t}, \mathrm{J}\left(\mathrm{CH}_{3}, \mathrm{CH}_{2}\right)=7.1, \mathrm{CH}_{2} \mathrm{CH}_{3}\right), 1.84$ $\left(3 \mathrm{H}, \mathrm{d}, J\left(\mathrm{CH}_{3}, 6\right)=1.2,5-\mathrm{CH}_{3}\right), 4.02\left(2 \mathrm{H}, \mathrm{q}, J\left(\mathrm{CH}_{2}, \mathrm{CH}_{3}\right)=7.1, \mathrm{OCH}_{2} \mathrm{CH}_{3}\right)$, $7.10\left(1 \mathrm{H}, \mathrm{m}, \mathrm{H}-4^{\prime}\right), 7.32\left(2 \mathrm{H}, \mathrm{m}, \mathrm{H}-3^{\prime}\right), 7.50\left(1 \mathrm{H}, \mathrm{q}, J\left(6, \mathrm{CH}_{3}\right)=1.2, \mathrm{H}-6\right)$, $7.52\left(2 \mathrm{H}, \mathrm{m}, \mathrm{H}-2^{\prime}\right), 8.53\left(1 \mathrm{H}\right.$, br s, H-3), $10.97\left(1 \mathrm{H}\right.$, br s, H-1). $\delta_{\mathrm{C}}$ (125.7 MHz, $\left.\mathrm{CDCl}_{3}\right) 8.87\left(5-\mathrm{CH}_{3}\right), 15.33\left(\mathrm{CH}_{2} \mathrm{CH}_{3}\right), 70.37\left(\mathrm{OCH}_{2} \mathrm{CH}_{3}\right)$, $106.78(\mathrm{C}-5), 120.55\left(\mathrm{C}-2^{\prime}\right), 124.13\left(\mathrm{C}-4^{\prime}\right), 128.91\left(\mathrm{C}-3^{\prime}\right), 137.40\left(\mathrm{C}-1^{\prime}\right)$, 152.00 (C-2), 157.83 (C-6), 169.87 (C-4). HRMS for $\mathrm{C}_{13} \mathrm{H}_{16} \mathrm{~N}_{2} \mathrm{O}_{3} \mathrm{Na}$ $(\mathrm{M}+\mathrm{Na})^{+}$calcd 271.1053, found 271.1055.

4.6.27. 1-(3-Ethoxy-2-methylacryloyl)-3-naphthalen-2-ylurea (9t). The title compound was prepared according to general method A from compound $\mathbf{8 m}(0.186 \mathrm{~g}, 1.3 \mathrm{mmol})$ and reagent $\mathbf{7 b}$ in $90 \%$ yield $(0.35 \mathrm{~g}, 1.174 \mathrm{mmol})$ of white solid. $\nu_{\max }(\mathrm{KBr}) 3268(\mathrm{~m})$, 3171 (w), 3150 (w), 3054 (w), 2986 (w), 2939 (w), 2898 (w), 1702 (vs), 1668 (s), 1655 (m, sh), 1617 (m), 1605 (m), 1588 (s), 1572 (s), 1512 (w), 1493 (w, sh), 1471 (m), 1396 (w), 1388 (w, sh), 1361 (w), 1286 (w), 1260 (w), 1216 (vs), 1180 (m), 1123 (s), 1042 (w), 1013 (w), $882(\mathrm{w}), 848(\mathrm{w}), 806(\mathrm{w}), 753(\mathrm{w}), 739(\mathrm{w}), 616(\mathrm{vw}), 469(\mathrm{w}) . \delta_{\mathrm{H}}$ $\left(499.8 \mathrm{MHz}, \mathrm{CDCl}_{3}\right) 1.26\left(3 \mathrm{H}, \mathrm{t}, J\left(\mathrm{CH}_{3}, \mathrm{CH}_{2}\right)=7.1, \mathrm{CH}_{2} \mathrm{CH}_{3}\right), 1.87$ (3H, d, $\left.J\left(\mathrm{CH}_{3}, 6\right)=1.2,5-\mathrm{CH}_{3}\right), 4.02\left(2 \mathrm{H}, \mathrm{q}, J\left(\mathrm{CH}_{2}, \mathrm{CH}_{3}\right)=7.1, \mathrm{OCH}_{2} \mathrm{CH}_{3}\right), 7.40$ $\left(1 \mathrm{H}, \mathrm{m}, \mathrm{H}-6^{\prime}\right), 7.46\left(1 \mathrm{H}, \mathrm{m}, \mathrm{H}-7^{\prime}\right), 7.52\left(1 \mathrm{H}, \mathrm{dd}, J\left(3^{\prime}, 4^{\prime}\right)=8.7\right.$, $\left.J\left(3^{\prime}, 1^{\prime}\right)=2.2, \mathrm{H}-3^{\prime}\right), 7.52\left(1 \mathrm{H}, \mathrm{q}, J\left(6, \mathrm{CH}_{3}\right)=1.2, \mathrm{H}-6\right), 7.77-7.81(3 \mathrm{H}, \mathrm{m}$, $\mathrm{H}-4^{\prime}, \mathrm{H}-5^{\prime}$ and $\left.\mathrm{H}-8^{\prime}\right), 8.15\left(1 \mathrm{H}, \mathrm{d}, J\left(1^{\prime}, 3^{\prime}\right)=2.1, \mathrm{H}-1^{\prime}\right), 8.37(1 \mathrm{H}, \mathrm{br} \mathrm{s}, \mathrm{H}-$ 3), $11.14\left(1 \mathrm{H}\right.$, br s, H-1). $\delta_{\mathrm{C}}\left(125.7 \mathrm{MHz}, \mathrm{CDCl}_{3}\right) 8.90\left(5-\mathrm{CH}_{3}\right), 15.28$ $\left(\mathrm{CH}_{2} \mathrm{CH}_{3}\right), 70.45\left(\mathrm{OCH}_{2} \mathrm{CH}_{3}\right), 106.57(\mathrm{C}-5), 117.08\left(\mathrm{C}-1^{\prime}\right), 120.58(\mathrm{C}-$ $\left.3^{\prime}\right), 124.92\left(\mathrm{C}-6^{\prime}\right), 126.43\left(\mathrm{C}-7^{\prime}\right), 127.54$ and $127.58\left(\mathrm{C}-5^{\prime}\right.$ and $\left.\mathrm{C}-8^{\prime}\right)$, 128.69 (C-4'), 130.60 (C-4'a), 133.86 (C-8'a), 134.89 (C-2'), 151.92 (C2), 158.03 (C-6), 169.80 (C-4). HRMS for $\mathrm{C}_{17} \mathrm{H}_{18} \mathrm{~N}_{2} \mathrm{O}_{3} \mathrm{Na}(\mathrm{M}+\mathrm{Na})^{+}$ calcd 321.1210, found 321.1209.

4.6.28. (R)-1-(Pyrrolidin-3-yl)uracil (10a). The title compound was prepared from 9a $(1.72 \mathrm{~g}, 5.254 \mathrm{mmol})$ according to general method E in $97 \%$ yield $(0.92 \mathrm{~g}, 5.08 \mathrm{mmol})$ of white solid. NMR and HRMS spectra were identical to those in Ref. 3.

4.6.29. 1-((3R,4S)-4-Hydroxypyrrolidin-3-yl)uracil (10b). The title compound was prepared from $\mathbf{9 b}(1.13 \mathrm{~g}, 1.75 \mathrm{mmol})$ according to general method $\mathrm{E}$ and was re-purified using preparative reversedphase HPLC in $90 \%$ yield $(0.31 \mathrm{~g}, 1.575 \mathrm{mmol})$ of white solid. $\nu_{\max }(\mathrm{KBr}) 3397$ (m), 3312 (m), 2599 (w, vbr), 1698 (vs), 1677 (s), 1620 (w, sh), 1467 (w), $1388(\mathrm{~m}), 1270(\mathrm{~m}), 1084(\mathrm{w}), 1053(\mathrm{w}), 802$ $(\mathrm{m}), 768(\mathrm{w}) . \delta_{\mathrm{H}}\left(600.1 \mathrm{MHz}, \mathrm{D}_{2} \mathrm{O}\right) 3.00\left(1 \mathrm{H}, \mathrm{dd}, J_{\text {gem }}=12.9\right.$,
$\left.J\left(5^{\prime} \mathrm{b}, 4^{\prime}\right)=2.8, \mathrm{H}-5^{\prime} \mathrm{b}\right), 3.24\left(1 \mathrm{H}, \mathrm{dd}, J_{g e m}=12.2, J\left(2^{\prime} \mathrm{b}, 3^{\prime}\right)=8.6, \mathrm{H}-2^{\prime} \mathrm{b}\right)$, $3.35\left(1 \mathrm{H}, \mathrm{dd}, J_{\text {gem }}=12.9, J\left(5^{\prime} \mathrm{a}, 4^{\prime}\right)=5.4, \mathrm{H}-5^{\prime} \mathrm{a}\right), 3.36(1 \mathrm{H}, \mathrm{dd}$, $\left.J_{g e m}=12.2, \quad J\left(2^{\prime} \mathrm{a}, 3^{\prime}\right)=8.4, \quad \mathrm{H}-2^{\prime} \mathrm{a}\right), 4.48 \quad\left(1 \mathrm{H}, \quad\right.$ ddd, $J\left(4^{\prime}, 3^{\prime}\right)=5.7$, $\left.J\left(4^{\prime}, 5^{\prime}\right)=5.4,2.8, \mathrm{H}-4^{\prime}\right), 4.90\left(1 \mathrm{H}, \mathrm{ddd}, J\left(3^{\prime}, 2^{\prime}\right)=8.6,8.4, J\left(3^{\prime}, 4^{\prime}\right)=5.7\right.$, $\left.\mathrm{H}-3^{\prime}\right), 5.82(1 \mathrm{H}, \mathrm{d}, J(5,6)=8.0, \mathrm{H}-5), 7.68(1 \mathrm{H}, \mathrm{d}, J(6,5)=8.0, \mathrm{H}-6) . \delta_{\mathrm{C}}$ (150.9 MHz, $\left.\mathrm{D}_{2} \mathrm{O}\right) 48.60\left(\mathrm{C}-2^{\prime}\right), 55.19\left(\mathrm{C}-5^{\prime}\right), 61.16\left(\mathrm{C}-3^{\prime}\right), 72.05\left(\mathrm{C}-4^{\prime}\right)$, 103.60 (C-5), 147.94 (C-6), 156.38 (C-2), 170.46 (C-4). HRMS for $\mathrm{C}_{8} \mathrm{H}_{12} \mathrm{~N}_{3} \mathrm{O}_{3}(\mathrm{M}+\mathrm{H})^{+}$calcd 198.0873, found 198.0873.

4.6.30. 1-((3S,4S)-4-Hydroxypyrrolidin-3-yl)uracil (10c). The title compound was prepared from $9 \mathrm{c}(0.4 \mathrm{~g}, 0.635 \mathrm{mmol})$ according to general method $\mathrm{E}$ in $80 \%$ yield $(0.1 \mathrm{~g}, 0.51 \mathrm{mmol})$ of white solid. $\nu_{\max }(\mathrm{KBr}) 3406$ (m, br), 3185 (m, vbr), 1687 (vs, br), 1628 (m, sh), $1459(\mathrm{~m}), 1420(\mathrm{~m}), 1382(\mathrm{~m}), 1270(\mathrm{~m}), 1078$ (w, br), $810(\mathrm{w}), 764$ (w). $\delta_{\mathrm{H}}\left(500.0 \mathrm{MHz}, \mathrm{D}_{2} \mathrm{O}\right) 2.94\left(1 \mathrm{H}, \mathrm{dd}, J_{\text {gem }}=12.3, J\left(5^{\prime} \mathrm{b}, 4^{\prime}\right)=5.2, \mathrm{H}-\right.$ $\left.5^{\prime} \mathrm{b}\right), 3.16\left(1 \mathrm{H}, \mathrm{dd}, J_{g e m}=12.7, J\left(2^{\prime} \mathrm{b}, 3^{\prime}\right)=5.9, \mathrm{H}-2^{\prime} \mathrm{b}\right), 3.38(1 \mathrm{H}, \mathrm{dd}$, $\left.J_{g e m}=12.3, J\left(5^{\prime} \mathrm{a}, 4^{\prime}\right)=5.9, \mathrm{H}-5^{\prime} \mathrm{a}\right), 3.51\left(1 \mathrm{H}, \mathrm{dd}, J_{g e m}=12.7, J\left(2^{\prime} \mathrm{a}, 3^{\prime}\right)=8.1\right.$, $\left.\mathrm{H}-2^{\prime} \mathrm{a}\right), 4.59\left(1 \mathrm{H}\right.$, ddd, $\left.J\left(4^{\prime}, 5^{\prime}\right)=5.9,5.2, J\left(4^{\prime}, 3^{\prime}\right)=2.7, \mathrm{H}-4^{\prime}\right), 4.61(1 \mathrm{H}$, ddd, $\left.J\left(3^{\prime}, 2^{\prime}\right)=8.1,5.9, J\left(3^{\prime}, 4^{\prime}\right)=2.7, \mathrm{H}-3^{\prime}\right), 5.83(1 \mathrm{H}, \mathrm{d}, J(5,6)=7.9, \mathrm{H}-5)$, $7.59(1 \mathrm{H}, \mathrm{d}, J(6,5)=7.9, \mathrm{H}-6) . \delta_{\mathrm{C}}\left(125.7 \mathrm{MHz}, \mathrm{D}_{2} \mathrm{O}\right) 50.55\left(\mathrm{C}-2^{\prime}\right), 54.68$ $\left(\mathrm{C}-5^{\prime}\right), 69.47\left(\mathrm{C}-3^{\prime}\right), 77.63\left(\mathrm{C}-4^{\prime}\right), 104.74(\mathrm{C}-5), 147.76(\mathrm{C}-6), 156.16(\mathrm{C}-$ 2), 170.98 (C-4). HRMS for $\mathrm{C}_{8} \mathrm{H}_{12} \mathrm{~N}_{3} \mathrm{O}_{3}(\mathrm{M}+\mathrm{H})^{+}$calcd 198.0873, found 198.0868 .

4.6.31. 1-Cyclopropyluracil (10d). The title compound was prepared from $9 d(0.45 \mathrm{~g}, 1.988 \mathrm{mmol})$ according to general method $\mathrm{E}$ in $97 \%$ yield $(0.35 \mathrm{~g}, 1.944 \mathrm{mmol})$ of white solid. $\nu_{\max }(\mathrm{KBr}) 3145(\mathrm{w}, \mathrm{br})$, 2998 (m, br), 2954 (m), 2876 (m), 1714 (s, sh), 1700 (vs), 1678 (vs), 1615 (m), 1472 (m), 1453 (w, sh), 1430 (w), 1421 (m), $1384(\mathrm{~m}), 1268$ (s), $765(\mathrm{w}) . \delta_{\mathrm{H}}\left(500.0 \mathrm{MHz}, \mathrm{DMSO}-d_{6}\right) 1.52-1.65$ (4H, m, H-2'a, 3'a, $4^{\prime} \mathrm{a}$ and $\left.\mathrm{H}-5^{\prime} \mathrm{a}\right), 1.75$ (2H, m, H-3'b and H-4'b), $1.91\left(2 \mathrm{H}, \mathrm{m}, \mathrm{H}-2^{\prime} \mathrm{b}\right.$ and $\left.\mathrm{H}-5^{\prime} \mathrm{b}\right), 4.70\left(1 \mathrm{H}, \mathrm{m}, \mathrm{H}-1^{\prime}\right), 5.56(1 \mathrm{H}, \mathrm{dd}, J(5,6)=8.0, J(5,3)=2.3, \mathrm{H}-5)$, $7.65(1 \mathrm{H}, \mathrm{d}, J(6,5)=8.0, \mathrm{H}-6), 11.22\left(1 \mathrm{H}\right.$, br s, H-3). $\delta_{\mathrm{C}}(125.7 \mathrm{MHz}$, DMSO $\left.-d_{6}\right) 23.84\left(\mathrm{C}-3^{\prime}\right.$ and $\left.\mathrm{C}-4^{\prime}\right), 30.72\left(\mathrm{C}-2^{\prime}\right.$ and $\left.\mathrm{C}-5^{\prime}\right), 56.50\left(\mathrm{C}-1^{\prime}\right)$, 101.59 (C-5), 142.74 (C-6), 151.32 (C-2), 163.51 (C-4). HRMS $\mathrm{C}_{9} \mathrm{H}_{13} \mathrm{~N}_{2} \mathrm{O}_{2}(\mathrm{M}+\mathrm{H})^{+}$calcd 181.0972, found 181.0972 .

4.6.32. 1-Cyclohexyluracil (10e). The title compound was prepared from $9 e(0.50 \mathrm{~g}, 2.08 \mathrm{mmol})$ according to general method $\mathrm{E}$ in $99 \%$ yield $(0.40 \mathrm{~g}, 2.061 \mathrm{mmol})$ of white solid. $\nu_{\max }(\mathrm{KBr}) 2930(\mathrm{~s}), 2865$ (m), 1714 (vs, sh), 1690 (vs, br), 1676 (vs, br), 1616 (s), 1470 (s), 1452 (m), $1428(\mathrm{~m}), 1419(\mathrm{~s}), 1379(\mathrm{~s}), 1268(\mathrm{~s}), 763(\mathrm{~m}) . \delta_{\mathrm{H}}(500.0 \mathrm{MHz}$, DMSO- $\left.d_{6}\right) 1.13\left(1 \mathrm{H}\right.$, qt, $J_{g e m}=J\left(4^{\prime} \mathrm{ax}, 3^{\prime} \mathrm{ax}\right)=J\left(4^{\prime} \mathrm{ax}, 5^{\prime} \mathrm{ax}\right)=13.0$, $\left.J\left(4^{\prime} \mathrm{ax}, 3^{\prime} \mathrm{eq}\right)=J\left(4^{\prime} \mathrm{ax}, 5^{\prime} \mathrm{eq}\right)=3.6, \quad \mathrm{H}^{\prime} 4^{\prime} \mathrm{ax}\right), \quad 1.32 \quad\left(2 \mathrm{H}, \quad \mathrm{qt}, \quad J_{g e m}=\right.$ $J\left(3^{\prime} \mathrm{ax}, 2^{\prime} \mathrm{ax}\right)=J\left(3^{\prime} \mathrm{ax}, 4^{\prime} \mathrm{ax}\right)=13.2, J\left(3^{\prime} \mathrm{ax}, 2^{\prime} \mathrm{eq}\right)=J\left(3^{\prime} \mathrm{ax}, 4^{\prime} \mathrm{eq}\right)=3.4, \mathrm{H}-3^{\prime} \mathrm{ax}$ and $\left.\mathrm{H}-5^{\prime} \mathrm{ax}\right), 1.54\left(2 \mathrm{H}, \mathrm{qd}, J_{g e m}=J\left(2^{\prime} \mathrm{ax}, 1^{\prime}\right)=J\left(2^{\prime} \mathrm{ax}, 3^{\prime} \mathrm{ax}\right)=12.4\right.$, $J\left(2^{\prime} \mathrm{ax}, 3^{\prime} \mathrm{eq}\right)=3.7, \mathrm{H}-2^{\prime} \mathrm{ax}$ and $\left.\mathrm{H}-6^{\prime} \mathrm{ax}\right), 1.61\left(1 \mathrm{H}, \mathrm{dm}, J_{\text {gem }}=12.9, \mathrm{H}-\right.$ $4^{\prime}$ eq), 1.69 (2H, m, H-2'eq and H-6'eq), $1.78\left(2 \mathrm{H}, \mathrm{dm}, J_{g e m}=13.5, \mathrm{H}-\right.$ $3^{\prime}$ eq and $\left.\mathrm{H}^{\prime} 5^{\prime} \mathrm{eq}\right), 4.23\left(1 \mathrm{H}, \quad \mathrm{tt}, \quad J\left(1^{\prime}, 2^{\prime} \mathrm{ax}\right)=J\left(1^{\prime}, 6^{\prime} \mathrm{ax}\right)=12.2\right.$, $J\left(1^{\prime}, 2^{\prime}\right.$ eq $)=J\left(1^{\prime}, 6^{\prime}\right.$ eq $\left.)=3.8, \mathrm{H}-1^{\prime}\right), 5.55(1 \mathrm{H}, \mathrm{dd}, J(5,6)=8.0, J(5,3)=2.3$, $\mathrm{H}-5), 7.70(1 \mathrm{H}, \mathrm{d}, J(6,5)=8.0, \mathrm{H}-6), 11.22(1 \mathrm{H}, \mathrm{br} \mathrm{s}, \mathrm{H}-3) . \delta_{\mathrm{C}}(125.7 \mathrm{MHz}$, DMSO- $\left.d_{6}\right) 24.85\left(C-4^{\prime}\right), 25.56\left(C-3^{\prime}\right.$ and $\left.C-5^{\prime}\right), 31.06\left(C-2^{\prime}\right.$ and $\left.C-6^{\prime}\right)$, 54.25 (C-1'), 101.33 (C-5), 142.40 (C-6), 151.10 (C-2), 163.43 (C-4). HRMS $\mathrm{C}_{10} \mathrm{H}_{14} \mathrm{~N}_{2} \mathrm{O}_{2} \mathrm{Na}(\mathrm{M}+\mathrm{Na})^{+}$calcd 217.0947, found 217.0948.

4.6.33. 1-(trans-2-Hydroxycyclohexyl)uracil (10f). The title compound was prepared from $9 f(0.33 \mathrm{~g}, 1.28 \mathrm{mmol})$ according to general method $\mathrm{E}$ in $78 \%$ yield $(0.210 \mathrm{~g}, 0.998 \mathrm{mmol})$ of white solid. $\nu_{\max }(\mathrm{KBr}) 3348(\mathrm{~s}), 3287$ (m, br, sh), 3162 (m, br), 3021 (m, br), 2937 (s), 2862 (m), 1728 (s), 1694 (vs, br), 1662 (s, sh), 1625 (s), 1474 (s), $1427(\mathrm{~m}), 1457(\mathrm{~m}), 1388(\mathrm{~s}), 1271(\mathrm{~s}), 1074(\mathrm{~m}), 764(\mathrm{w}) . \delta_{\mathrm{H}}$ (499.8 MHz, DMSO-d $d_{6}$ ) 1.21-1.29 (3H, m, H-3'a, 4'a and H-5'a), 1.56 ( $1 \mathrm{H}$, br s, H-6'a), 1.62-1.71 (3H, m, H-4'b, 5'b and $\left.\mathrm{H}^{\prime}-6^{\prime} \mathrm{b}\right), 1.94(1 \mathrm{H}$, $\left.\mathrm{m}, \mathrm{H}-3^{\prime} \mathrm{b}\right), 3.64\left(1 \mathrm{H}, \mathrm{br} \mathrm{s}, \mathrm{H}-2^{\prime}\right), 4.06\left(1 \mathrm{H}, \mathrm{br} \mathrm{s}, \mathrm{H}-1^{\prime}\right), 4.89(1 \mathrm{H}, \mathrm{br} \mathrm{d}$, $\left.J\left(\mathrm{OH}, 2^{\prime}\right)=5.0, \mathrm{OH}\right), 5.54(1 \mathrm{H}, \mathrm{d}, J(5,6)=7.9, \mathrm{H}-5), 7.67(1 \mathrm{H}, \mathrm{d}$, $J(6,5)=7.9, \mathrm{H}-6), 11.12\left(1 \mathrm{H}\right.$, br s, H-3). $\delta_{\mathrm{C}}\left(125.7 \mathrm{MHz}, \mathrm{DMSO}-d_{6}\right)$ 
$24.02\left(\mathrm{C}-4^{\prime}\right), 25.02\left(\mathrm{C}-5^{\prime}\right), 30.08\left(\mathrm{C}-6^{\prime}\right), 35.14\left(\mathrm{C}-3^{\prime}\right), 60.36\left(\mathrm{C}-1^{\prime}\right)$, 69.00 (C-2'), 100.98 (C-5), 142.86 (C-6), 151.61 (C-2), 163.46 (C-4). HRMS for $\mathrm{C}_{10} \mathrm{H}_{15} \mathrm{~N}_{2} \mathrm{O}_{3}(\mathrm{M}+\mathrm{H})^{+}$calcd 211.1077, found 211.1078.

4.6.34. 1-(cis-2-Hydroxycyclopentyl)uracil (10g). The title compound was prepared from $9 \mathrm{~g}(0.28 \mathrm{~g}, 1.155 \mathrm{mmol})$ according to general method $\mathrm{E}$ in $95 \%$ yield $(0.215 \mathrm{~g}, 0.153 \mathrm{mmol})$ of white solid. $\nu_{\max }(\mathrm{KBr})$ 3470 (m), 3418 (m, br), 3288 (w, br, sh), 3165 (m), 3024 (m, br), 2964 (m), 2873 (w), 1701 (vs), 1691 (vs), 1665 (s, sh), 1619 (m), 1473 (m), 1453 (w, sh), 1425 (w), 1393 (w), 1277 (m), 1015 (w), $762(\mathrm{w}) . \delta_{\mathrm{H}}$ (499.8 MHz, DMSO- $\left.d_{6}\right)$ 1.48-1.61 (2H, m, H-3'a and H-4'a), 1.75-1.83 ( $2 \mathrm{H}, \mathrm{m}, \mathrm{H}-4^{\prime} \mathrm{b}$ and $\left.\mathrm{H}-5^{\prime} \mathrm{a}\right), 1.85-1.95$ (2H, m, H-3'b and $\left.\mathrm{H}-5^{\prime} \mathrm{b}\right), 4.03$ $\left(1 \mathrm{H}, \mathrm{m}, \mathrm{H}-2^{\prime}\right), 4.50\left(1 \mathrm{H}, \mathrm{m}, \mathrm{H}-1^{\prime}\right), 4.93\left(1 \mathrm{H}, \mathrm{br} \mathrm{d}, J\left(\mathrm{OH}, 2^{\prime}\right)=4.2, \mathrm{OH}\right)$, $5.48(1 \mathrm{H}, \mathrm{dd}, J(5,6)=8.0, J(5,3)=2.3, \mathrm{H}-5), 7.60(1 \mathrm{H}, \mathrm{d}, J(6,5)=8.1, \mathrm{H}-6)$, $11.16\left(1 \mathrm{H}\right.$, br s, H-3). $\delta_{\mathrm{C}}\left(125.7 \mathrm{MHz}, \mathrm{DMSO}-d_{6}\right) 20.05\left(\mathrm{C}-4^{\prime}\right), 26.05$ (C5'), 32.79 (C-3'), 58.56 (C-1'), 69.62 (C-2'), 99.63 (C-5), 144.22 (C-6), $151.71(\mathrm{C}-2), 163.56(\mathrm{C}-4)$. HRMS for $\mathrm{C}_{9} \mathrm{H}_{12} \mathrm{~N}_{2} \mathrm{O}_{3} \mathrm{Na}(\mathrm{M}+\mathrm{Na})^{+}$calcd 219.0740, found 219.0741.

4.6.35. 1-(1-(Hydroxymethyl)cyclopentyl)uracil (10h). The title compound was prepared from $9 \mathrm{~h}(0.53 \mathrm{~g}, 2.067 \mathrm{mmol})$ according to general method E in $92 \%$ yield $(0.402 \mathrm{~g}, 1.914 \mathrm{mmol})$ of white solid. $\nu_{\max }(\mathrm{KBr}) 3404(\mathrm{~s}), 3176(\mathrm{~m}), 3054(\mathrm{~m}, \mathrm{br}), 2956(\mathrm{~m}), 2879(\mathrm{~m}), 1705$ (vs), 1689 (vs), 1662 (vs), 1617 (w), 1465 (m), 1415 (m), 1387 (m), 1265 (w), $1062(\mathrm{~m}), 764(\mathrm{~m}) . \delta_{\mathrm{H}}\left(500.0 \mathrm{MHz}, \mathrm{DMSO}-d_{6}\right) 1.55\left(2 \mathrm{H}, \mathrm{m}, \mathrm{H}-3^{\prime} \mathrm{a}\right.$ and $\left.\mathrm{H}-4^{\prime} \mathrm{a}\right), 1.65\left(2 \mathrm{H}, \mathrm{m}, \mathrm{H}-3^{\prime} \mathrm{b}\right.$ and $\left.\mathrm{H}-4^{\prime} \mathrm{b}\right), 1.78\left(2 \mathrm{H}, \mathrm{m}, \mathrm{H}-2^{\prime} \mathrm{a}\right.$ and H-5'a ), 2.15 (2H, m, H-2'b and $\mathrm{H}^{\prime} 5^{\prime} \mathrm{b}$ ), 3.49 ( $2 \mathrm{H}, \mathrm{s}, \mathrm{CH}_{2} \mathrm{O}$ ), 5.06 (1H, br s, $\mathrm{OH}), 5.41(1 \mathrm{H}, \mathrm{dd}, J(5,6)=8.1, J(5,3)=2.5, \mathrm{H}-5), 7.47(1 \mathrm{H}, \mathrm{d}, J(6,5)=8.2$, $\mathrm{H}-6), 11.01(1 \mathrm{H}$, br d, $J(3,5)=2.2, \mathrm{H}-3) . \delta_{\mathrm{C}}\left(125.7 \mathrm{MHz}\right.$, DMSO-d $\left.\mathrm{d}_{6}\right) 22.35$ $\left(\mathrm{C}-3^{\prime}\right.$ and $\left.\mathrm{C}-4^{\prime}\right), 33.78\left(\mathrm{C}-2^{\prime}\right.$ and $\left.\mathrm{C}-5^{\prime}\right), 61.59\left(\mathrm{CH}_{2} \mathrm{OH}\right), 73.42\left(\mathrm{C}-1^{\prime}\right)$, 99.49 (C-5), 145.75 (C-6), 151.37 (C-2), 164.00 (C-4). HRMS $\mathrm{C}_{10} \mathrm{H}_{14} \mathrm{~N}_{2} \mathrm{O}_{3} \mathrm{Na}(\mathrm{M}+\mathrm{Na})^{+}$calcd 233.0897, found 233.0896.

4.6.36. 1-(2,3-Dihydroxypropyl)uracil (10i). The title compound was prepared from $9 \mathbf{i}(0.37 \mathrm{~g}, 1.59 \mathrm{mmol})$ according to general method $\mathrm{E}$ in $97 \%$ yield $(0.29 \mathrm{~g}, 1.559 \mathrm{mmol})$ of white solid. $\nu_{\max }(\mathrm{KBr}) 3424$ (br), 3375 (s, br), 3150 (m, br, sh), 3101 (m), 1689 (vs, sh), 1663 (vs, br), 1620 (s), $1469(\mathrm{~m}), 1429(\mathrm{~s}), 1391(\mathrm{~m}), 1250(\mathrm{~m}), 1085$ or $1068(\mathrm{~m}), 1047(\mathrm{~m})$, $764(\mathrm{~m}) . \delta_{\mathrm{H}}\left(500.0 \mathrm{MHz}\right.$, DMSO-d $\left.d_{6}\right) 3.29\left(1 \mathrm{H}, \mathrm{dd}, J_{\text {gem }}=11.1\right.$, $\left.J\left(3^{\prime} \mathrm{a}, 2^{\prime}\right)=5.9, \mathrm{H}-3^{\prime} \mathrm{a}\right), 3.36\left(1 \mathrm{H}, \mathrm{dd}, J_{g e m}=11.1, J\left(3^{\prime} \mathrm{b}, 2^{\prime}\right)=5.2, \mathrm{H}-3^{\prime} \mathrm{b}\right), 3.36$ $\left(1 \mathrm{H}, \mathrm{dd}, J_{\text {gem }}=13.7, J\left(1^{\prime} \mathrm{a}, 2^{\prime}\right)=8.8, \mathrm{H}-1^{\prime} \mathrm{a}\right), 3.67\left(1 \mathrm{H}, \mathrm{m}, \mathrm{H}-2^{\prime}\right), 3.92(1 \mathrm{H}$, dd, $\left.J_{\text {gem }}=13.7, J\left(1^{\prime} \mathrm{b}, 2^{\prime}\right)=3.5, \mathrm{H}-1^{\prime} \mathrm{b}\right), 5.50(1 \mathrm{H}, \mathrm{dd}, J(5,6)=7.8$, $J(5,3)=2.3, \mathrm{H}-5), 7.49(1 \mathrm{H}, \mathrm{d}, J(6,5)=7.9, \mathrm{H}-6), 11.22(1 \mathrm{H}, \mathrm{br} \mathrm{d}$, $J(3,5)=2.1, \mathrm{H}-3) . \delta_{\mathrm{C}}\left(125.7 \mathrm{MHz}, \mathrm{DMSO}-d_{6}\right) 51.38\left(\mathrm{C}-1^{\prime}\right), 63.92\left(\mathrm{C}-3^{\prime}\right)$, 69.30 (C-2'), 100.28 (C-5), 147.36 (C-6), 151.40 (C-2), 164.25 (C-4). HRMS $\mathrm{C}_{7} \mathrm{H}_{10} \mathrm{~N}_{2} \mathrm{O}_{4} \mathrm{Na}(\mathrm{M}+\mathrm{Na})^{+}$calcd 209.0533, found 209.0534.

4.6.37. 1-Phenyluracil (10j). The title compound was prepared from $\mathbf{9 j}(0.4 \mathrm{~g}, 0.635 \mathrm{mmol})$ according to general method $\mathrm{E}$ in $96 \%$ yield (0.17 g, $0.903 \mathrm{mmol}$ ) of white solid. $\nu_{\max }(\mathrm{KBr}) 3159$ (w, br), $1779(\mathrm{~m})$, $1745(\mathrm{~s}), 1716(\mathrm{~m}), 1692$ (vs), $1663(\mathrm{~m}, \mathrm{sh}), 1628(\mathrm{~m}), 1601(\mathrm{~m}), 1579$ (w, sh), $1494(\mathrm{w}), 1460(\mathrm{w}), 1440(\mathrm{~m}), 1423(\mathrm{~m}), 1384$ (s), 1321 (vw), 1258 (m), 1182 (vw), 1075 (w), 1034 (w), 999 (w), 909 (w), $824(\mathrm{~m}), 757$ $(\mathrm{m}), 688(\mathrm{~m}), 507(\mathrm{w}) . \delta_{\mathrm{H}}\left(500.0 \mathrm{MHz}, \mathrm{CDCl}_{3}\right) 5.83(1 \mathrm{H}, \mathrm{dd}, J(5,6)=8.0$, $J(5,3)=2.2, \mathrm{H}-5), 7.34(1 \mathrm{H}, \mathrm{d}, J(6,5)=8.0, \mathrm{H}-6), 7.35(2 \mathrm{H}, \mathrm{m}, \mathrm{H}-o-\mathrm{Ph})$, $7.45(1 \mathrm{H}, \mathrm{m}, \mathrm{H}-\mathrm{p}-\mathrm{Ph}), 7.50(2 \mathrm{H}, \mathrm{m}, \mathrm{H}-m-\mathrm{Ph}), 8.39(1 \mathrm{H}, \mathrm{br} \mathrm{s}, \mathrm{H}-3) . \delta_{\mathrm{C}}$ (125.7 MHz, CDCl $) 102.65$ (C-5), 126.26 (C-o-Ph), 129.05 (C-p-Ph), 129.70 (C-m-Ph), 138.32 (C-i-Ph), 144.64 (C-6), 149.96 (C-2), 162.95 (C-4). HRMS for $\mathrm{C}_{10} \mathrm{H}_{9} \mathrm{~N}_{2} \mathrm{O}_{2}(\mathrm{M}+\mathrm{H})^{+}$calcd 189.0659 , found 189.0658 .

4.6.38. Methyl 2-(1-uracilyl)acetate (10k). The title compound was prepared from $9 \mathbf{k}(0.74 \mathrm{~g}, 3.21 \mathrm{mmol})$ according to general method $\mathrm{E}$ in $78 \%$ yield $(0.46 \mathrm{~g}, 2.5 \mathrm{mmol})$ of white solid. $\nu_{\max }(\mathrm{KBr}) 3183(\mathrm{~m}$, br), 1739 (vs), 1700 (vs, br), 1634 (s), 1454 (s), 1440 (m, sh), 1419 (m), $1381(\mathrm{~m}), 1250(\mathrm{~s}, \mathrm{sh}), 1235(\mathrm{~s}), 1223(\mathrm{~s}), 822(\mathrm{~m}), 768(\mathrm{~m}), 760(\mathrm{~m})$. $\delta_{\mathrm{H}}\left(500.0 \mathrm{MHz}, \mathrm{DMSO}-d_{6}\right) 3.68\left(3 \mathrm{H}, \mathrm{s}, \mathrm{CH}_{3} \mathrm{O}\right), 4.52\left(2 \mathrm{H}, \mathrm{s}, \mathrm{CH}_{2} \mathrm{~N}\right)$,
$5.62(1 \mathrm{H}, \mathrm{dd}, J(5,6)=7.9, J(5,3)=2.2, \mathrm{H}-5), 7.61(1 \mathrm{H}, \mathrm{d}, J(6,5)=7.9, \mathrm{H}-$ $6), 11.42(1 \mathrm{H}$, br d, $J(3,5)=2.2, \mathrm{H}-3) . \delta_{\mathrm{C}}\left(125.7 \mathrm{MHz}, \mathrm{DMSO}-d_{6}\right) 48.33$ $\left(\mathrm{CH}_{2} \mathrm{~N}\right), 52.62\left(\mathrm{CH}_{3} \mathrm{O}\right), 101.42(\mathrm{C}-5), 146.18(\mathrm{C}-6), 151.25(\mathrm{C}-2), 164.09$ (C-4), 168.97 (CO). HRMS for $\mathrm{C}_{7} \mathrm{H}_{8} \mathrm{~N}_{2} \mathrm{O}_{4} \mathrm{Na}(\mathrm{M}+\mathrm{H}+\mathrm{Na})^{+}$calcd 207.0376, found 207.0377.

4.6.39. (R)-Ethyl 2-(1-uracilyl)propanoate (10l). The title compound was prepared from $91(0.73 \mathrm{~g}, 2.83 \mathrm{mmol})$ according to general method $\mathrm{E}$ in $96 \%$ yield $(0.58 \mathrm{~g}, 2.73 \mathrm{mmol})$ of white solid. $\nu_{\max }(\mathrm{KBr})$ 3150 (m, br), 1754 (s), 1746 (vs), 1713 (vs), 1672 (vs), 1617 (s, sh), $1480(\mathrm{~m}), 1471(\mathrm{~m}), 1459(\mathrm{~m}), 1415(\mathrm{~m}), 1399(\mathrm{~m}), 1380(\mathrm{~s}), 1272(\mathrm{~s})$, 1203 (s, sh), 1191 (s), 1115 (w), 1097 (w, sh), 830 (m), 822 (m), 679 $(\mathrm{w}), 760(\mathrm{w}) . \delta_{\mathrm{H}}\left(500.0 \mathrm{MHz}, \mathrm{DMSO}-d_{6}\right) 1.17\left(3 \mathrm{H}, \mathrm{t}, J\left(\mathrm{CH}_{3}, \mathrm{CH}_{2}\right)=7.1\right.$, $\left.\mathrm{CH}_{3} \mathrm{CH}_{2} \mathrm{O}\right), 1.52\left(3 \mathrm{H}, \mathrm{d}, \mathrm{J}\left(\mathrm{CH}_{3}, \mathrm{CH}\right)=7.3, \mathrm{CH}_{3}\right), 4.13\left(2 \mathrm{H}, \mathrm{m}, \mathrm{CH}_{3} \mathrm{CH}_{2} \mathrm{O}\right)$, $4.98\left(1 \mathrm{H}, \mathrm{q}, J\left(\mathrm{CH}_{1} \mathrm{CH}_{3}\right)=7.3, \mathrm{CH}\right), 5.60(1 \mathrm{H}, \mathrm{dd}, J(5,6)=8.0$, $J(5,3)=2.2, \mathrm{H}-5), 7.66(1 \mathrm{H}, \mathrm{d}, J(6,5)=8.0, \mathrm{H}-6), 11.32(1 \mathrm{H}, \mathrm{br} \mathrm{s}, \mathrm{H}-3)$. $\delta_{\mathrm{C}}\left(125.7 \mathrm{MHz}\right.$, DMSO- $\left.d_{6}\right) 14.16\left(\mathrm{CH}_{3} \mathrm{CH}_{2} \mathrm{O}\right), 15.46\left(\mathrm{CH}_{3}\right), 54.94$ (CH), $61.46\left(\mathrm{CH}_{3} \mathrm{CH}_{2} \mathrm{O}\right), 101.38$ (C-5), 144.15 (C-6), $150.92(\mathrm{C}-2)$, 163.58 (C-4), 170.19 (CO). HRMS for $\mathrm{C}_{9} \mathrm{H}_{12} \mathrm{~N}_{2} \mathrm{O}_{4} \mathrm{Na}(\mathrm{M}+\mathrm{H}+\mathrm{Na})^{+}$ calcd 235.0689, found 235.0689.

4.6.40. (R)-1-(Pyrrolidin-3-yl)thymine (10m). The title compound was prepared from $9 \mathrm{~m}(0.79 \mathrm{~g}, 2.314 \mathrm{mmol})$ according to general method E in $93 \%$ yield $(0.42 \mathrm{~g}, 2.15 \mathrm{mmol})$ of white solid. NMR and HRMS spectra were identical to those in Ref. 3.

4.6.41. 1-((3R,4S)-4-Hydroxypyrrolidin-3-yl)thymine (10n). The title compound was prepared from 9 n $(0.52 \mathrm{~g}, 0.79 \mathrm{mmol})$ according to general method $\mathrm{E}$ and was re-purified using preparative reverse phase HPLC in $90 \%$ yield $(0.15 \mathrm{~g}, 0.71 \mathrm{mmol})$ of white solid. NMR and HRMS spectra were identical to those in Ref. 4.

4.6.42. 1-Cyclohexylthymine (10o). The title compound was prepared from $90(0.27 \mathrm{~g}, 1.06 \mathrm{mmol})$ according to general method $\mathrm{E}$ in $95 \%$ yield $(0.21 \mathrm{~g}, 1.008 \mathrm{mmol})$ of white solid. $\nu_{\max }(\mathrm{KBr}) 3395(\mathrm{w})$, 3167 (s), 3100 (s, br), 3042 (s, br), 2931 (s), 2858 (s), 1692 (vs, br), 1661 (vs, br), 1517 (w), 1473 (s), 1451 (s), 1419 (m), 1392 (s), 1368 (m), 1271 (vs), $1046(\mathrm{w}), 759(\mathrm{~m}) . \delta_{\mathrm{H}}\left(500.0 \mathrm{MHz}, \mathrm{CDCl}_{3}\right) 1.18(1 \mathrm{H}, \mathrm{m}$, H-4'ax), 1.39-1.50 (4H, m, H-2'ax, 3'ax, 5'ax and H-6'ax), $1.74(1 \mathrm{H}$, $\mathrm{dm}, J_{\text {gem }}=13.2, \mathrm{H}-4^{\prime}$ eq $), 1.86-1.92\left(4 \mathrm{H}, \mathrm{m}, \mathrm{H}-2^{\prime}\right.$ eq, $3^{\prime}$ eq, $4^{\prime}$ eq and $\mathrm{H}-$ $\left.6^{\prime} \mathrm{eq}\right), 1.93\left(3 \mathrm{H}, \mathrm{d}, J\left(\mathrm{CH}_{3}, 6\right)=1.2, \mathrm{CH}_{3}\right), 4.46\left(1 \mathrm{H}, \mathrm{m}, \mathrm{H}-1^{\prime}\right), 7.06(1 \mathrm{H}, \mathrm{q}$, $\left.J\left(6, \mathrm{CH}_{3}\right)=1.2, \mathrm{H}-6\right), 9.34(1 \mathrm{H}, \mathrm{br} \mathrm{s}, \mathrm{H}-3) . \delta_{\mathrm{C}}\left(125.7 \mathrm{MHz}, \mathrm{CDCl}_{3}\right) 12.54$ $\left(\mathrm{CH}_{3}\right), 25.12\left(\mathrm{C}-4^{\prime}\right), 25.53\left(\mathrm{C}-3^{\prime}\right.$ and $\left.\mathrm{C}-5^{\prime}\right), 31.87\left(\mathrm{C}-2^{\prime}\right.$ and $\left.\mathrm{C}-6^{\prime}\right), 54.33$ (C-1'), 110.44 (C-5), 136.51 (C-6), 151.03 (C-2), 163.79 (C-4). HRMS $\mathrm{C}_{11} \mathrm{H}_{17} \mathrm{~N}_{2} \mathrm{O}_{2}(\mathrm{M}+\mathrm{H})^{+}$calcd 209.1285, found 209.1284.

4.6.43. 1-(trans-2-Hydroxycyclohexyl)thymine (10p). The title compound was prepared from 9p $(0.25 \mathrm{~g}, 0.924 \mathrm{mmol})$ according to general method $\mathrm{E}$ in $96 \%$ yield $(0.20 \mathrm{~g}, 0.892 \mathrm{mmol})$ of white solid. $\nu_{\max }(\mathrm{KBr}) 3459$ (s), 3335 (w, br, sh), 3150 (m, br), 3021 (m, br), 2939 (m), 2858 (m), 1696 (vs, sh), 1685 (vs), 1663 (vs), 1514 (w), 1477 (m), $1454(\mathrm{~m}), 1428(\mathrm{~m}), 1390(\mathrm{~m}), 1374(\mathrm{~m}), 1271(\mathrm{~s}), 1068(\mathrm{~m}), 1046(\mathrm{w}$, $\mathrm{sh}), 764(\mathrm{w}), 758(\mathrm{w}) . \delta_{\mathrm{H}}\left(499.8 \mathrm{MHz}, \mathrm{DMSO}-d_{6}\right) 1.20-1.31(3 \mathrm{H}, \mathrm{m}$, H-3'a, 4'a and H-5'a), 1.58 (1H, br s, H-6'a), 1.62-1.71 (3H, m, H-4'b, $5^{\prime} \mathrm{b}$ and $\left.\mathrm{H}-6^{\prime} \mathrm{b}\right), 1.77\left(3 \mathrm{H}, \mathrm{br}, \mathrm{CH}_{3}\right), 1.94\left(1 \mathrm{H}, \mathrm{m}, \mathrm{H}-3^{\prime} \mathrm{b}\right), 3.65(1 \mathrm{H}, \mathrm{br}$, $\left.\mathrm{H}-2^{\prime}\right), 4.06(1 \mathrm{H}$, br s, H-1' $), 4.73(1 \mathrm{H}, \mathrm{br} \mathrm{s}, \mathrm{OH}), 7.56(1 \mathrm{H}$, br s, H-6), $11.07\left(1 \mathrm{H}\right.$, br s, H-3). $\delta_{\mathrm{C}}\left(125.7 \mathrm{MHz}\right.$, DMSO- $\left.d_{6}\right) 12.29\left(\mathrm{CH}_{3}\right), 24.07$ $\left(\mathrm{C}-4^{\prime}\right), 25.05\left(\mathrm{C}-5^{\prime}\right), 30.09\left(\mathrm{C}-6^{\prime}\right), 35.14\left(\mathrm{C}-3^{\prime}\right), 59.45\left(\mathrm{C}-1^{\prime}\right), 68.97$ (C-2'), 108.53 (C-5), 138.47 (C-6), 151.58 (C-2), 164.03 (C-4). HRMS $\mathrm{C}_{11} \mathrm{H}_{17} \mathrm{~N}_{2} \mathrm{O}_{3}(\mathrm{M}+\mathrm{H})^{+}$calcd 225.1234, found 225.1234.

4.6.44. 1-(1-(Hydroxymethyl)cyclopentyl)thymine (10q). The title compound was prepared from $\mathbf{9 q}(0.30 \mathrm{~g}, 0.90 \mathrm{mmol})$ according to general method $\mathrm{E}$ in $97 \%$ yield $(0.195 \mathrm{~g}, 0.870 \mathrm{mmol})$ of white solid. $\nu_{\max }(\mathrm{KBr}) 3428(\mathrm{~m}, \mathrm{sh}), 3355(\mathrm{~s}), 3150(\mathrm{~m}), 3090(\mathrm{~m}), 3027(\mathrm{~s}), 2954$ (s), 2880 (m), 1716 (vs), 1664 (vs), 1478 (m), 1465 (m), 1451 (m), 
$1424(\mathrm{~m}), 1389$ (m), 1365 (m), 1268 (m), 1054 (s), 1037 (m), 763 (m), $758(\mathrm{~m}) . \delta_{\mathrm{H}}\left(499.8 \mathrm{MHz}, \mathrm{DMSO}-d_{6}\right) 1.55\left(2 \mathrm{H}, \mathrm{m}, \mathrm{H}-3^{\prime} \mathrm{a}\right.$ and H-4'a), $1.65\left(2 \mathrm{H}, \mathrm{m}, \mathrm{H}-3^{\prime} \mathrm{b}\right.$ and $\left.\mathrm{H}-4^{\prime} \mathrm{b}\right), 1.75\left(3 \mathrm{H}, \mathrm{d}, J\left(\mathrm{CH}_{3}, 6\right)=1.1, \mathrm{CH}_{3}\right), 1.80$ ( $2 \mathrm{H}, \mathrm{m}, \mathrm{H}-2^{\prime} \mathrm{a}$ and $\left.\mathrm{H}-5^{\prime} \mathrm{a}\right), 2.17$ (2H, m, H-2'b and $\left.\mathrm{H}^{\prime} 5^{\prime} \mathrm{b}\right), 3.50(2 \mathrm{H}, \mathrm{d}$, $\left.J\left(\mathrm{CH}_{2}, \mathrm{OH}\right)=5.9, \mathrm{CH}_{2} \mathrm{O}\right), 4.97\left(1 \mathrm{H}, \mathrm{t}, J\left(\mathrm{OH}, \mathrm{CH}_{2}\right)=5.9, \mathrm{OH}\right), 7.33(1 \mathrm{H}, \mathrm{q}$, $\left.J\left(6, \mathrm{CH}_{3}\right)=1.2, \mathrm{H}-6\right), 10.96\left(1 \mathrm{H}\right.$, br s, H-3). $\delta_{\mathrm{C}}\left(125.7 \mathrm{MHz}, \mathrm{DMSO}-d_{6}\right)$ $12.37\left(\mathrm{CH}_{3}\right), 22.34\left(\mathrm{C}-3^{\prime}\right.$ and $\left.\mathrm{C}-4^{\prime}\right), 33.83\left(\mathrm{C}-2^{\prime}\right.$ and $\left.\mathrm{C}-5^{\prime}\right), 61.60$ $\left(\mathrm{CH}_{2} \mathrm{OH}\right), 73.07\left(\mathrm{C}-1^{\prime}\right), 106.71$ (C-5), 141.34 (C-6), $151.25(\mathrm{C}-2)$, 164.42 (C-4). HRMS $\mathrm{C}_{11} \mathrm{H}_{17} \mathrm{~N}_{2} \mathrm{O}_{3}(\mathrm{M}+\mathrm{H})^{+}$calcd 225.1234, found 225.1234 .

4.6.45. 1-(2,3-Dihydroxypropyl)thymine (10r). The title compound was prepared from 9r $(0.19 \mathrm{~g}, 0.90 \mathrm{mmol})$ according to general method $\mathrm{E}$ in $97 \%$ yield $(0.195 \mathrm{~g}, 0.870 \mathrm{mmol})$ of white solid. $\nu_{\max }(\mathrm{KBr}) 3494$ (s), 3392 (s, br), 3280 (m, br, sh), 3145 (m, br), 1700 (s, sh), 1678 (vs), 1642 (s, sh), 1520 (vw), 1482 (m), 1429 (m), 1389 $(\mathrm{m}), 1096(\mathrm{~s}), 1044(\mathrm{~m}), 763(\mathrm{~m}) . \delta_{\mathrm{H}}\left(499.8 \mathrm{MHz}, \mathrm{DMSO}-d_{6}\right) 1.74(3 \mathrm{H}$, $\left.\mathrm{d}, J\left(\mathrm{CH}_{3}, 6\right)=1.2, \mathrm{CH}_{3}\right), 3.30\left(1 \mathrm{H}, \mathrm{dd}, J_{g e m}=11.1, J\left(3^{\prime} \mathrm{a}, 2^{\prime}\right)=5.8, \mathrm{H}-3^{\prime} \mathrm{a}\right)$, $3.36\left(1 \mathrm{H}, \mathrm{dd}, J_{g e m}=11.1, J\left(3^{\prime} \mathrm{b}, 2^{\prime}\right)=5.2, \mathrm{H}-3^{\prime} \mathrm{b}\right), 3.36\left(1 \mathrm{H}, \mathrm{dd}, J_{g e m}=13.7\right.$, $\left.J\left(1^{\prime} \mathrm{a}, 2^{\prime}\right)=8.6, \mathrm{H}^{\prime} 1^{\prime} \mathrm{a}\right), 3.68\left(1 \mathrm{H}, \mathrm{m}, \mathrm{H}-2^{\prime}\right), 3.87\left(1 \mathrm{H}, \mathrm{dd}, J_{g e m}=13.7\right.$, $\left.J\left(1^{\prime} \mathrm{b}, 2^{\prime}\right)=3.6, \mathrm{H}-1^{\prime} \mathrm{b}\right), 7.38\left(1 \mathrm{H}, \mathrm{q}, J\left(6, \mathrm{CH}_{3}\right)=1.2, \mathrm{H}-6\right), 11.17(1 \mathrm{H}$, br s, $\mathrm{H}-3) . \delta_{\mathrm{C}}\left(125.7 \mathrm{MHz}\right.$, DMSO-d $\left.d_{6}\right) 12.18\left(\mathrm{CH}_{3}\right), 51.11\left(\mathrm{C}-1^{\prime}\right), 63.88(\mathrm{C}-$ $\left.3^{\prime}\right), 69.34\left(\mathrm{C}-2^{\prime}\right), 107.66$ (C-5), 143.11 (C-6), 151.31 (C-2), 164.65 (C-4). HRMS $\mathrm{C}_{8} \mathrm{H}_{11} \mathrm{~N}_{2} \mathrm{O}_{4}(\mathrm{M}+\mathrm{H})^{+}$calcd 199.0713, found 199.0722.

4.6.46. 1-Phenylthymine (10s). The title compound was prepared from 9s $(0.26 \mathrm{~g}, 1.04 \mathrm{mmol})$ according to general method $\mathrm{E}$ in $97 \%$ yield $(0.205 \mathrm{~g}, 1.014 \mathrm{mmol})$ of white solid. $\nu_{\max }(\mathrm{KBr}) 3166(\mathrm{~s}, \mathrm{br})$, 3101 (m), 3038 (s, br), 1700 (vs, br), 1664 (vs, br), 1650 (vs, br), 1595 (vs), 1508 (m), 1491 (vs), 1478 (s), 1469 (s), 1446 (s), 1432 (s), 1419 (s), 1385 (m), 1371 (s), 1318 (s), 1279 (vs), 1172 (m), 1156 (w), 1080 (w), $1036(\mathrm{~s}), 1006(\mathrm{w}), 965(\mathrm{w}), 916(\mathrm{~s}), 841(\mathrm{~m}), 761(\mathrm{~s}), 692(\mathrm{~s}), 615$ $(\mathrm{w}), 515(\mathrm{~m}) . \delta_{\mathrm{H}}\left(499.8 \mathrm{MHz}, \mathrm{CDCl}_{3}\right) 1.97\left(3 \mathrm{H}, \mathrm{d}, J\left(\mathrm{CH}_{3}, 6\right)=1.3, \mathrm{CH}_{3}\right)$, $7.18\left(1 \mathrm{H}, \mathrm{q}, J\left(6, \mathrm{CH}_{3}\right)=1.3, \mathrm{H}-6\right), 7.34\left(2 \mathrm{H}, \mathrm{m}, \mathrm{H}-2^{\prime}\right), 7.41\left(1 \mathrm{H}, \mathrm{m}, \mathrm{H}-4^{\prime}\right)$, $7.48\left(2 \mathrm{H}, \mathrm{m}, \mathrm{H}-3^{\prime}\right), 9.09(1 \mathrm{H}, \mathrm{br} \mathrm{s}, \mathrm{H}-3) . \delta_{\mathrm{C}}\left(125.7 \mathrm{MHz}, \mathrm{CDCl}_{3}\right) 12.23$ $\left(\mathrm{CH}_{3}\right), 111.08(\mathrm{C}-5), 126.28\left(\mathrm{C}-2^{\prime}\right), 128.66\left(\mathrm{C}-4^{\prime}\right), 129.51\left({\left.\mathrm{C}-3^{\prime}\right)}^{\prime}, 138.57\right.$ $\left(\mathrm{C}-1^{\prime}\right), 140.66$ (C-6), 150.26 (C-2), 164.15 (C-4). HRMS for $\mathrm{C}_{11} \mathrm{H}_{11} \mathrm{~N}_{2} \mathrm{O}_{2}$ $(\mathrm{M}+\mathrm{H})^{+}$calcd 203.0815, found 203.0815 .

4.6.47. 1-(2-Naphthyl)thymine (10t). The title compound was prepared from $9 t(0.33 \mathrm{~g}, 1.106 \mathrm{mmol})$ according to general method $\mathrm{E}$ in $97 \%$ yield $(0.27 \mathrm{~g}, 1.071 \mathrm{mmol})$ of white solid. $\nu_{\max }(\mathrm{KBr}) 3170(\mathrm{~s}$, br), 3049 (s, br), 1704 (vs, br), 1684 (vs, br), 1655 (vs, sh), 1631 (s), 1598 (s), 1508 (s), 1468 (s), 1455 (s), 1437 (s), 1423 (s), 1373 (s), 1377 (s), 1359 (m), 1293 (vs), $1272(\mathrm{~s}), 1142(\mathrm{w}), 1127$ (m), 1043 (m), 1019 (w), $955(\mathrm{w}), 946(\mathrm{w}), 887(\mathrm{~m}, \mathrm{sh}), 866(\mathrm{~s}), 810(\mathrm{~s}), 772(\mathrm{~m}), 765(\mathrm{~s})$, $753(\mathrm{~s}), 649(\mathrm{~m}), 629(\mathrm{w}), 527(\mathrm{w}), 477(\mathrm{~s}) . \delta_{\mathrm{H}}\left(499.8 \mathrm{MHz}, \mathrm{CDCl}_{3}\right)$ $2.04\left(3 \mathrm{H}, \mathrm{d}, J\left(\mathrm{CH}_{3}, 6\right)=1.3, \mathrm{CH}_{3}\right), 7.29\left(1 \mathrm{H}, \mathrm{q}, J\left(6, \mathrm{CH}_{3}\right)=1.3, \mathrm{H}-6\right), 7.46$ $\left(1 \mathrm{H}, \mathrm{dd}, J\left(3^{\prime}, 4^{\prime}\right)=8.7, J\left(3^{\prime}, 1^{\prime}\right)=2.2, \mathrm{H}-3^{\prime}\right), 7.54-7.58\left(2 \mathrm{H}, \mathrm{m}, \mathrm{H}-6^{\prime}\right.$ and $\left.\mathrm{H}-7^{\prime}\right), 7.80\left(1 \mathrm{H}, \mathrm{d}, J\left(1^{\prime}, 3^{\prime}\right)=2.3, \mathrm{H}-1^{\prime}\right), 7.85-7.91\left(2 \mathrm{H}, \mathrm{m}, \mathrm{H}-5^{\prime}\right.$ and $\mathrm{H}-$ $\left.8^{\prime}\right), 7.95\left(1 \mathrm{H}, \mathrm{d}, J\left(4^{\prime}, 3^{\prime}\right)=8.7, \mathrm{H}-4^{\prime}\right), 8.60\left(1 \mathrm{H}\right.$, br s, H-3). $\delta_{\mathrm{C}}$ $\left(125.7 \mathrm{MHz}, \mathrm{CDCl}_{3}\right) 12.30\left(\mathrm{CH}_{3}\right), 111.20(\mathrm{C}-5), 124.05\left(\mathrm{C}-3^{\prime}\right), 124.87$ $\left(\mathrm{C}-1^{\prime}\right), 127.11$ and $127.19\left(\mathrm{C}-6^{\prime}\right.$ and $\left.\mathrm{C}-7^{\prime}\right), 127.83$ and $128.01\left(\mathrm{C}-5^{\prime}\right.$ and C-8'), 129.59 (C-4'), 132.78 (C-4'a), 133.26 (C-8'a), 136.06 (C-2'), 140.88 (C-6), 150.29 (C-2), 163.92 (C-4). HRMS for $\mathrm{C}_{15} \mathrm{H}_{13} \mathrm{~N}_{2} \mathrm{O}_{2}$ $(\mathrm{M}+\mathrm{H})^{+}$calcd 253.0972, found 253.0972.

4.6.48. Methyl 3-ethoxy-2-methylacryloylcarbamate (11). A sample of $\mathbf{7 b}$ was dissolved in $5 \mathrm{ml}$ of anhydrous methanol. Few drops of triethylamine was added. The mixture was evaporated and dissolved in $\mathrm{CDCl}_{3} . \quad \delta_{\mathrm{H}}\left(500.0 \mathrm{MHz}, \mathrm{CDCl}_{3}\right) 1.33(3 \mathrm{H}, \mathrm{t}$, $\left.J\left(\mathrm{CH}_{3}, \mathrm{CH}_{2}\right)=7.1, \mathrm{CH}_{3}\right), 1.83\left(3 \mathrm{H}, \mathrm{d}, J\left(\mathrm{CH}_{3}, 6\right)=1.2,5-\mathrm{CH}_{3}\right), 3.79(3 \mathrm{H}, \mathrm{s}$, $\left.\mathrm{CH}_{3} \mathrm{O}\right), \quad 4.08\left(2 \mathrm{H}, \mathrm{q}, J\left(\mathrm{CH}_{2}, \mathrm{CH}_{3}\right)=7.1, \mathrm{OCH}_{2} \mathrm{CH}_{3}\right), 7.42(1 \mathrm{H}, \mathrm{q}$, $\left.J\left(6, \mathrm{CH}_{3}\right)=1.2, \mathrm{H}-6\right), 7.79\left(1 \mathrm{H}\right.$, br s, H-3). $\delta_{\mathrm{C}}\left(125.7 \mathrm{MHz}, \mathrm{CDCl}_{3}\right) 9.07$ (5- $\left.\mathrm{CH}_{3}\right), 15.26\left(\mathrm{CH}_{3}\right), 52.72\left(\mathrm{CH}_{3} \mathrm{O}\right), 70.12\left(\mathrm{OCH}_{2} \mathrm{CH}_{3}\right), 107.09(\mathrm{C}-5)$, 151.93 (C-2), 157.59 (C-6), 165.97 (C-4). HRMS for $\mathrm{C}_{8} \mathrm{H}_{13} \mathrm{NO}_{4} \mathrm{Na}$ $(\mathrm{M}+\mathrm{H}+\mathrm{Na})^{+}$calcd 210.0742, found 210.0741 .

\section{Acknowledgements}

Support by grants No. NR/9138 - 3 (Ministry of Health, CR), No. 2B06065, and research centra LC06077 and LC06061 (Ministry of Education, CR), KAN200520801 (Acad. Sci. CR), and 203/09/0820 (Czech Science Foundation) under the Institute research project Z40550506 is gratefully acknowledged. Authors are indebted to the staff of the Department of Mass Spectroscopy for measurements of HRMS and MALDI-TOF.

\section{References and notes}

1. Rejman, D.; Masojídková, M.; De Clercq, E.; Rosenberg, I. Nucleosides Nucleotides 2001, 20, 1497-1522.

2. Kočalka, P.; Pohl, R.; Rejman, D.; Rosenberg, I. Nucleosides Nucleotides Nucleic Acids 2005, 24, 805-808.

3. Kočalka, P.; Pohl, R.; Rejman, D.; Rosenberg, I. Tetrahedron 2006, 62, 5763-5774.

4. Rejman, D.; Kočalka, P.; Buděšínský, M.; Pohl, R.; Rosenberg, I. Tetrahedron 2007, 63, 1243-1253.

5. Shaw, G.; Warrener, R. N. J. Chem. Soc. 1958, 153-156; Shaw, G.; Warrener, R. N. J. Chem. Soc. 1958, 157-161.

6. Shealy, Y. F.; O'Dell, C. A. J. Heterocycl. Chem. 1976, 13, 1015-1020.

7. Wyatt, P. G.; Anslow, A. S.; Coomber, B. A.; Cousins, R. P. C.; Evans, D. N.; Gilbert, V. S.; Humber, D. C.; Paternoster, I. L.; Sollis, S. L.; Tapolczay, D. J.; Weingarten, G. G. Nucleosides Nucleotides 1995, 14, 2039-2049.

8. Hřebabecký, H.; Masojídková, M.; Holý, A. Collect. Czech. Chem. Commun. 2005, 70, 519-538.

9. Csuk, R.; von Scholz, Y. Tetrahedron 1995, 51, 7193-7206.

10. Shealy, Y. F.; O'Dell, C. A. J. Heterocycl. Chem. 1976, 13, 1041-1047.

11. Borthwick, A. D.; Evans, D. N.; Kirk, B. E.; Biggadike, K.; Exal, A. M.; Youds, P.; Roberts, S. M.; Knight, D. J.; Coates, A. V. J. Med. Chem. 1990, 33, 179-186.

12. Bodenteich, M.; Marquez, V. E.; Barchi, J. J.; Hallows, W. H.; Goldstein, B. M.; Driscoll, J. S. J. Org. Chem. 1993, 58, 6009-6015.

13. Hosono, F.; Nishiyama, S.; Yamamura, S. Tetrahedron 1994, 50, 13335-13346.

14. Raganathan, S.; George, K. S. Tetrahedron 1997, 53, 3347-3362.

15. Miyabe, H.; Kanehira, S.; Kume, K.; Kandori, H.; Naito, T. Tetrahedron 1998, 54, 5883-5892.

16. Moon, H. R.; Kim, H. O.; Chun, M. W.; Jeong, L. S. J. Org. Chem. 1999, 64, 4733-4741.

17. Wang, P.; Gullen, B.; Newton, M. G.; Cheng, Y. C.; Shinazi, R. F.; Chu, C. K. J. Med. Chem. 1999, 42, 3390-3399.

18. Bera, S.; Mickle, T.; Nair, V. Nucleosides Nucleotides 1999, 18, 2379-2395.

19. Kim, H. S.; Ravi, R. G.; Marquez, V. E.; Maddileti, S.; Wihlborg, A. K.; Erlinge, D.; Malmsjö, M.; Boyer, J. L.; Harden, T. K.; Jacobson, K. A. J. Med. Chem. 2002, 45, 208-218.

20. Nieto, M. I.; Caamaño, O.; Fernández, F.; Gómez, M.; Balzarini, J.; DeClercq, E. Nucleosides Nucleotides 2002, 21, 243-255.

21. Kim, S. A.; Lee, H. M.; Ryu, J. S.; Kim, H. S. Synlett 2007, 1055-1058.

22. Migliore, M. D.; Zonta, N.; McGuigan, C.; Henson, G.; Andrei, G.; Snoeck, R.; Balzarini, J. J. Med. Chem. 2007, 50, 6485-6492.

23. Terán, C.; Teijera, M.; Santana, L.; Uriarte, E.; Castiñeiras, A. J. Mol. Struct. 1998, 448, 69-75.

24. Liboska, R.; Masojídková, M.; Rosenberg, I. Collect. Czech. Chem. Commun. 1996, 61, 313-332.

25. Liboska, R.; Masojídková, M.; Rosenberg, I. Collect. Czech. Chem. Commun. 1996, 61, 778-790.

26. Hakala, H.; Ollikka, P.; Degerholm, J.; Hovinen, J. Tetrahedron 2002, 58, 8771-8777.

27. Horhota, A. T.; Szostak, J. W.; McLaughlin, L. W. Org. Lett. 2006, 23, 5345-5347.

28. Tao, L.; Yue, Y.; Zhang, J.; Chen, S.; Yu, X. Helv. Chim. Acta 2008, 91, 1008-1014.

29. Qu, G.; Zhang, Z.; Guo, H.; Geng, M.; Xia, R. Molecules 2007, 12, 543-551. 


\title{
The Stability and Reactivity of Activated Acryloylcarbamates as Reagents \\ for the Synthesis of $N-1$ Substituted Thymine and Uracil - An NMR and DFT Study
}

\author{
Radek Pohl, ${ }^{*[a]}$ Lubomír Rulíšek, ${ }^{*[a]}$ and Dominik Rejman*a] \\ [a] Institute of Organic Chemistry and Biochemistry AS CR, v.v.i., Flemingovo nám. 2, 16610 Prague 6, Czech Republic \\ Fax: +420 220183123 \\ E-mail: pohl@uochb.cas.cz; rulisek@uochb.cas.cz; rejman@uochb.cas.cz
}

Keywords: uracil / thymine / nucleosidation / mechanism / NMR / DFT

\begin{abstract}
:
The mechanism of the decomposition of acryloylcarbamates $\mathbf{7 a - b}$ yielding highly reactive isocyanates 3a-b was proposed based on NMR measurements and quantum chemical calculations. A good agreement between the experimental kinetic data and DFT calculations allowed us to demonstrate that the stability of 7a-d depends on the presence of methyl in the acryloyl moiety and the position of the nitro group in the nitrophenolic part of the molecule. Furthermore, the reactivity of 7a-d with weakly nucleophilic and sterically hindered 2,4,6-tritert-butylaniline was explored by ${ }^{1} \mathrm{H}$ NMR demonstrating the usefulness of reagents $7 \mathbf{a}-\mathbf{d}$ offering access to a variety of $1-N$-substituted uracils and thymines with potentially interesting biological properties.
\end{abstract}

\section{Introduction}

Many biologically active compounds belong to the class of nucleosides and nucleotides analogues, which play an important role in anticancer therapy ${ }^{1-2}$ or act as antiviral compounds. ${ }^{3}$ Specific examples also involve carbocyclic nucleosides ${ }^{4-5}$ (Carbovir®) and acyclic phosphonate nucleotides ${ }^{6}$ (Viread, Cidofovir, Tenofovir, Hepsera), which represent biologically important compounds in which the nucleobase is not connected via a glycosidic bond.

From a synthetic point of view, a crucial step in their preparation is the attachment of the nucleobase - the nucleosidation reaction. The most common synthetic routes to purine 
nucleoside analogues are represented by direct alkylation of the appropriate nucleobases (adenine, 6-chloropurine or 2-amino-6-chloropurine) with the halo, tosyloxy or mesyloxy derivatives in dipolar aprotic solvents (DMF, DMSO) using cesium carbonate as a base. ${ }^{7-9}$ Alternatively, Mitsunobu reaction of 6-chloropurine or 2-amino-6-chloropurine with appropriate hydroxyderivative can be used. ${ }^{8,10-11}$ Recently, $N^{6}$-bis-Boc-protected adenine was successfully used as a substrate for the Mitsunobu reaction during the synthesis of neplanocin A. ${ }^{12}$ However, all of these methods for the introduction of pyrimidine nucleobases (uracil, thymine, and cytosine) suffer from both low regioselectivity and low yield of the 1-Nsubstituted product. The direct alkylation of nucleobases is usually accompanied by a competing elimination reaction, which further decreases the yield of the desired product. ${ }^{8-9}$ Some improvement (both in regioselectivity and overall yield) can be obtained through the use of 2,4-dimethoxypyrimidine or 2,4-dimethoxy-6-methylpyrimidine for the alkylation instead of uracil or thymine, respectively. ${ }^{13-15}$ As an alternative approach to the pyrimidine nucleoside analogues (e.g. carbocyclic nucleosides), a synthesis of 1-N-substituted nucleobases starting from a primary amine and isocyanates $\mathbf{3 a - b}$ or carbamates $\mathbf{6 a - b}$ were described fifty years ago by Shaw and Warrener. ${ }^{16}$ The key isocyanates 3a-b, accessible through two independent routes ${ }^{17-18}$ (Scheme 1), were used as intermediates for the synthesis of carbamates 6a-b described by Hřebabecký et al. ${ }^{19}$

Scheme 1. The reagents for the preparation of $1-N$-substituted pyrimidine nucleobases.

Isocyanates 3a-b are an unusually moisture-sensitive species and also very reactive compounds, capable of forming carbamates with free hydroxy groups. ${ }^{20}$ Therefore, the reaction is usually carried out either with the appropriately protected substrate or without protection at low temperature $\left(<-20^{\circ} \mathrm{C}\right)$. This property seems to be the main factor responsible for the varying yield of the reported uracil and thymine derivatives (usually $\sim 20$ $80 \%$, in most cases $<60 \%$ ) found in the literature. ${ }^{21-32}$ The formation of uracil and thymine rings is a two-stage reaction consisting of the aminolysis of carbamates $\mathbf{6 a - b}$ or the addition of amine to isocyanates $\mathbf{3 a - b}$ followed by a cyclization of the acryloylureas $\mathbf{9}$ formed (Scheme 2). ${ }^{33}$

Scheme 2. The reaction of reagents $\mathbf{3 , 6}$ and $\mathbf{7}$ with amines and subsequent cyclization providing $1-N$-substituted pyrimidines. 
To date, there has not been any general, high-yield method available for the attachment of uracil and thymine moieties to sterically hindered secondary, tertiary, or even aromatic carbon atoms.

It was the extreme reactivity and moisture sensitivity of isocyanates $\mathbf{3}$ on the one hand and the low yields of $\mathbf{9}$ obtained using carbamates $\mathbf{6}$ on the other that prompted us to develop more advantageous reagents 7 , which proved to be reactive enough with a variety of amines. Moreover, they can be stored for longer time (several months in refrigerator), which enabled us to use them in modular syntheses of uracils and thymines $10^{34}$ The synthesis of the reagents $7 \mathbf{a}-\mathbf{d}$ followed the procedure described for $\mathbf{6},{ }^{19}$ in whose last step of the 'one-pot' synthesis, a 2-nitrophenol solution in dioxane was added instead of ethanol.

The reagents $\mathbf{7 a}-\mathbf{d}$ were tested in reactions with a wide variety of amines (including those bonded to secondary, tertiary, and aromatic systems), giving consistently high yields of corresponding 1,3-disubstituted urea derivatives. The desired uracil and thymine derivatives were obtained by the cyclization of the urea intermediates using Dowex 50 in $\mathrm{H}^{+}$form, providing again very high yields. ${ }^{34}$

In this work, we wish to address an unprecedented decomposition of the reagent $\mathbf{7 b}$ in $\mathrm{CDCl}_{3}$ solution observed in the ${ }^{1} \mathrm{H}$ NMR measurements, to analyze and quantitatively characterize the reaction mechanism of the decomposition reaction. To this end, we conducted a series of NMR experiments to yield reliable kinetic data and a series of DFT calculations to provide a plausible mechanistic view of the studied reaction. In order to understand the reaction mechanism in detail, we have compared the experimental data with other reagents $7 \mathbf{a}, 7 \mathbf{c}$ and 7d. We have also investigated the reaction of 7a-d with low nucleophilic and sterically hindered 2,4,6-tri-tert-butylaniline to ensure that decomposition of 7a-b does not limit their synthetic usage in preparation of $N-1$ substituted thymines and uracils. These findings represent a useful and successful conjunction of theoretical and experimental efforts extending our understanding of the organic reactions pertinent to this class of compounds.

\section{Results and Discussion}

\section{Kinetics of decomposition studied by NMR}

Reagents 7a-d were prepared according Scheme 1 and fully characterized by ${ }^{1} \mathrm{H}$ and ${ }^{13} \mathrm{C}$ NMR, elemental analysis, IR and MS. In the course of the NMR characterization of $\mathbf{7 b}$, we found that the reagent decomposes, yielding a complex mixture. The ${ }^{1} \mathrm{H}$ NMR spectrum of $\mathbf{7 b}$ 
Scheme 3. The decomposition of $\mathbf{7 b}$ via hydrolysis and decarboxylation.

To rule out the effect of water, which might be present in regular $\mathrm{CDCl}_{3}$, the ${ }^{1} \mathrm{H} \mathrm{NMR}$ spectra were recorded in dried $\mathrm{CDCl}_{3}$ and a sealed NMR tube (see Experimental). The experiment has again shown the decomposition of compound $\mathbf{7 b}$, and the final products of decomposition were identified as acryloylisocyanate $\mathbf{3 b}$ and 2-nitrophenol. Reagent $\mathbf{7 b}$ was characterised by NMR only when a high concentration of sample and a short measurement time were applied.

To ascertain that acryloylisocyanate $\mathbf{3 b}$ is the decomposition product, we allowed the decomposed mixture to react with dry methanol, yielding methyl ester 12, i.e. the same product as that formed through the reaction of $\mathbf{7 b}$ with methanol in the presence of $\mathrm{Et}_{3} \mathrm{~N}$ (Scheme 4). In addition, isocyanate $\mathbf{3 b}$ was identified by IR measured in dry $\mathrm{CHCl}_{3}$ (the characteristic band being at $2244 \mathrm{~cm}^{-1}$ for asymmetric stretching vibrations of the -NCO group) and by ${ }^{13} \mathrm{C}$ NMR (with the characteristic chemical shift of -NCO carbon at 130.47 ppm).

Scheme 4. The chemical proof of $\mathbf{3 b}$ structure.

The rate of decomposition was estimated based on the ${ }^{1} \mathrm{H}$ NMR kinetic measurement in dry $\mathrm{CDCl}_{3}$ and sealed NMR tube at $25{ }^{\circ} \mathrm{C}$. The concentrations of starting compounds and decomposition product(s) were obtained by integration of several corresponding signals with similar relaxation times. The rate constant for the irreversible first order kinetic model was obtained from plots of the logarithm of the starting compound concentrations versus time, while rate constant for the reversible first order kinetic model was obtained from plots of the logarithm of the starting compound concentrations minus equilibrium concentration versus time and from equilibrium constant. Experimental data for decomposition of $\mathbf{7 b}$ fitted irreversible reaction with first order kinetics (Table 1 and Supporting Information) with half life $\left(t_{1 / 2}\right)$ of about 18 minutes. The kinetics measurements were also performed for the other 
reagents, 7a, 7c and 7d, and the results have been summarized in Table 1. The decomposition of 7a was reversible reaction with first order kinetics (Table 1 and Supporting Information), while compounds $\mathbf{7 c}$ and $\mathbf{7 d}$ were stable and did not decompose at all.

As arises from Table 1, the kinetic measurements demonstrate a surprisingly strong dependence on the reagents and their structure. Reagents $\mathbf{7 c}$ and $\mathbf{7 d}$ (possessing a nitro group in the para position) do not undergo any degradation. On the other hand, the derivatives with the ortho-nitro group in nitrophenol moiety decompose readily, with the decomposition of the 7b-bearing methyl group in the acryloyl part of the molecule being approximately fifty times faster than the decomposition of the 7a-lacking methyl substituent. The different stability of the reaction products reflected in the molecular structure was further explored by DFT calculations.

\section{Reaction mechanism of decomposition. Theoretical calculations.}

We first optimised all sixteen planar geometries for each of $\mathbf{7 a - d}$, viz. all the possible arrangements of four double or partial double bonds in the system (64 molecular geometries in total) at the DFT(PBE)/def2-SVP level with the single-point energy calculated at the DFT(B3LYP)/def2-TZVP level. The solvation effects and corrections to the ZPE energy, enthalpy and entropy (using the ideal gas approximation) were included as well (for details, see Experimental). The results of this extensive conformational search are summarised in Tables S1-S5 (see Supporting Information). As can be seen in Tables S1-S4, the calculations predict (in all cases) the most stable isomers to possess trans-configuration on the $-\mathrm{C}=\mathrm{C}$ double bond. This fact has been corroborated experimentally by a missing NOE interaction between the Me group and the proton on the double bond for 7a--b or by a missing NOE between the protons of the double bond for $\mathbf{7} \mathbf{c}-\mathbf{d}$. At the same time we observe NOE between protons of $\mathrm{CH}_{2}$ group from $\mathrm{EtO}$ and $\mathrm{H}$ or $\mathrm{CH}_{3}$ on double bond in alpha position to $\mathrm{CO}$.

With regard to the proposed reaction mechanism, we have also investigated the possible isomers with the proton on either of the oxygens adjacent to the central - $\mathrm{NH}$ - group (these calculations were carried out only for the most stable isomers). The results are also listed in Tables S1-S4 (including various terms in overall Gibbs energies), and all twenty-four structures of $\mathbf{7 b}$ (assuming an $E$ configuration on the $-\mathrm{C}=\mathrm{C}$ - double bond) have been schematically depicted in Fig. 1. 
Figure 1. The planar geometries of $E-\mathbf{7 b}$ investigated by density functional theory calculations.

The calculations have shown that the $2 \mathrm{NH}-\mathrm{ZZZ}$ conformer is the most stable structure for the keto-tautomer of $\mathbf{7 b}$ whereas $3 \mathrm{OH}-\mathrm{ZZE}$ is the lowest energy enol-form of the same compound. It can be noted that $3 \mathrm{OH}-\mathrm{ZZE}$ geometry is nicely preorganised to form a six-membered transition state with bifurcated hydrogen bonding.

However, a rather surprising finding is the relatively small difference in energies between all the studied isomers. Considering the planar models depicted in Fig. 1, some of the structures would have been expected to have substantial intramolecular crowding. Even for such structures (e.g. 2NH-ZEE, 2NH-EEE) the energy or free energy differences amount to only $\sim 15-25 \mathrm{~kJ} \mathrm{~mol}^{-1}$. This demonstrates a relatively high level of flexibility in the studied molecules (the systems avoid crowding by adopting a helical form). Thus, we had to consider several reaction pathways for the elimination reaction. The lowest saddle points and their energies are summarised in Table 2 for all studied compounds (7a-7d) and for the proposed reaction mechanism of degradation shown in Scheme 5. It can be mentioned that all saddle points are characterized by a single imaginary frequency corresponding to the reactive coordinate and are therefore true transition states for the studied reactions. By small changes of the geometry in the direction of reactant and product we ascertained that it connects the desired reactant and products structures (IRC-like calculation). A more complete listing of the calculated values of activation barriers can be found in Tables S1-S4.

Scheme 5 . The degradation of $\mathbf{7 b}-\mathbf{b}$ proposed mechanism.

Furthermore, the reaction coordinate with the structures of all the key points on the potential energy surface is depicted in Figure 2.

Figure 2. The reaction coordinate for the degradation of $E-\mathbf{7 b}$. 
As mentioned above, the transition state is characterised by the six-membered ring arrangement and is structurally close to the enol-form with activation barriers of 72-88 $\mathrm{kJ}^{\mathrm{mol}}{ }^{-1}$ (Table 2). We have also investigated an alternative pathway starting from the more stable keto-form and involving the direct hydrogen transfer from the nitrogen atom to the oxygen atom of the nitrophenol ring. The activation barrier involving a four-membered transition state was considerably higher ( 160-180 kJ.mol ${ }^{-1}$, see Tables S1, S3, S4) for the 2NH-EEZ isomer (which has the most favourable arrangement of the atoms and highest thermodynamic stability of the reactants). This clearly favours the pathways involving the sixmembered transition states described above.

As arises from Table 2, the presence of the ortho-nitro group plays an important role in the bifurcated hydrogen bond and lowers the free enthalpy of degradation $\Delta G_{\text {calc. On the other }}$ hand, the methyl group on the $-\mathrm{C}=\mathrm{C}$ - double bond lowers the free energy of activation, $\Delta G_{\text {calc }}^{\ddagger}$, likely due to the effect of hyperconjugation. The computational results are in good agreement with the experimentally calculated $\Delta G_{\text {exp }}^{\ddagger}$ computed from the rate constants using Eyring equation. Since the accurate ab initio determination of the absolute rate constant is beyond the scope of this work (for a thorough discussion on the subject, we refer the reader to Ref. 35), we mostly want to highlight the excellent agreement in the relative difference between the two reactions $(\mathbf{7 a}, \mathbf{7 b})$ for which the comparison is available. $\Delta \Delta G_{\exp }^{\ddagger}=9.7$ $\mathrm{kJ} . \mathrm{mol}^{-1}$, whereas $\Delta \Delta G_{\text {calc }}^{\ddagger}=11.9 \mathrm{~kJ} \cdot \mathrm{mol}^{-1}$.

\section{NMR characterisation of the reactivity of $7 \mathrm{a}-\mathrm{d}$ towards amines}

In our previous work, ${ }^{34}$ we showed that reagents $7 \mathbf{a}, \mathbf{b}$ react with a variety of amines, including acyclic, cyclic, and aromatic, and also amino acids. All of the reactions were completed within one hour at room temperature. Nevertheless, the reaction kinetics were not addressed in detail. Herein, the reactivity of $\mathbf{7 a - d}$ with low nucleophilic and sterically hindered 2,4,6-tri-tert-butylaniline is presented (Scheme 6).

Scheme 6. The reaction of 7a-d with 2,4,6-tri-tert-butylaniline.

The kinetic measurements were performed in dry $\mathrm{CDCl}_{3}$ at $25{ }^{\circ} \mathrm{C}$ and monitored by ${ }^{1} \mathrm{H} \mathrm{NMR}$. The concentrations of starting compounds and products were obtained by integration of 


\section{Conclusions}

It has been shown that the studied reagents $7 \mathbf{a}-\mathbf{d}$ are highly reactive even with lownucleophilic and sterically hindered amines and can be successfully applied for a modular synthesis of various $1-N$-substituted uracils and thymines, which is difficult to achieve with other synthetic methods. The stability of reagents $\mathbf{7 a - d}$ in dry $\mathrm{CDCl}_{3}$ was investigated by experimental and theoretical methods. It has been proved that the decomposition of the studied molecules is strongly affected by the presence of the Me-group on the $-\mathrm{C}=\mathrm{C}$ - double bond and the position of the nitro group on the phenol ring. While the former is assumed to lower the activation barrier of the reaction probably due to hyperconjugation, the latter plays a role in hydrogen bonding and influences the thermodynamics of the reaction. The experimental NMR observations are in good agreement with the results predicted by the DFT calculations, which allowed us to postulate a plausible reaction mechanism, presumably extending our understanding of the reactivity and stability of this class of compounds. It should be noted here that all the reagents are stable enough in the solid state to be stored for a long time. Moreover, the decomposition of the 2-nitrophenyl derivatives $\mathbf{7 a}$ and $\mathbf{7 b}$ yields an 
isocyanate product that could react in the same manner as the parent reagents. These compounds could be seen as an 'instant form' of the previously described isocyanates 3a-b.

\section{Experimental Section}

NMR Experiments: NMR spectra were acquired on a Bruker Avance 500 spectrometer (500.0 MHz for ${ }^{1} \mathrm{H}$ and $125.7 \mathrm{MHz}$ for ${ }^{13} \mathrm{C}$ ) in dry $\mathrm{CDCl}_{3} . \mathrm{CDCl}_{3}$ was dried prior to use by anhydrous $\mathrm{K}_{2} \mathrm{CO}_{3}$ and distilled. The measurements were performed in an oven-dried NMR tube, which was sealed after sample preparation. The concentration of the samples was 38 $\mathrm{mM}$ and $40 \mathrm{mM}$ for the degradation kinetics and kinetics of reaction with amines, respectively. All of the kinetic measurements were performed at $25{ }^{\circ} \mathrm{C}$. Temperature calibration was done using the standard method with an $\mathrm{MeOH}$ sample. ${ }^{1} \mathrm{H}$ and ${ }^{13} \mathrm{C}$ resonances were assigned based on PFG H,C-HSQC and H,C-HMBC experiments (for numbering see Supportin Info). ${ }^{1} \mathrm{H}$ and ${ }^{13} \mathrm{C}$ NMR of compounds 7a-d have been already reported. ${ }^{34}$

Computational Details: All the density functional theory (DFT) calculations reported in the study were carried out using the Turbomole 6.0 program. ${ }^{36}$ The Perdew-Burke-Ernzerhof $(\mathrm{PBE})^{37}$ and hybrid three-parameter Becke's ${ }^{38}$ (B3LYP) functionals were used throughout. The calculations were expedited by expanding the Coulomb integrals in an auxiliary basis set, the resolution-of-identity (RI-J) approximation. ${ }^{39-40}$ All the geometry optimisations were conducted using the def2-SVP basis set, ${ }^{41}$ whereas the single-point energies were recomputed in the def2-TZVP (triple-zeta valence with two polarisation functions on each atom). ${ }^{42}$

To account for the solvation effects, the conductor-like screening model (COSMO) method ${ }^{41-}$ ${ }^{42}$ was used with the dielectric constant corresponding to chloroform $\left(\varepsilon_{\mathrm{r}}=4.9\right)$. In order to account for dispersion, we used the DFT+D method (i.e. the DFT method with the empirical dispersion terms) available in Turbomole 6.0. ${ }^{43}$ The Gibbs free energy was then calculated as the sum of these contributions:

$G=E_{\mathrm{el}}+G_{\mathrm{solv}}+E_{\mathrm{ZPE}}-R T \ln \left(q_{\mathrm{trans}} q_{\mathrm{rot}} q_{\mathrm{vib}}\right)$

where $E_{\mathrm{el}}$ is the in vacuo energy of the system (at the B3LYP/def2-TZVP level and the geometry optimised at the RI-PBE/def2-SVP level), $G_{\text {solv }}$ is the solvation free energy (at the RI-PBE/def2-SVP level), $E_{\mathrm{ZPE}}$ is the zero-point energy, and $-R T \ln \left(q_{\text {trans }} q_{\mathrm{rot}} q_{\mathrm{vib}}\right)$ accounts for the entropic terms and the thermal correction to the enthalpy obtained from a frequency 
calculation using the same method and software as for the geometry optimisation at the RIPBE/def2-SV(P) level, $298 \mathrm{~K}$, and 1 atm using the ideal-gas approximation. ${ }^{44}$

\section{(E)-3-Ethoxy-2-methylacryloyl isocyanate $(3 b)$}

${ }^{1} \mathrm{H}$ NMR $\left(\mathrm{CDCl}_{3}, 500.0 \mathrm{MHz}\right): 1.36 \mathrm{t}, 3 \mathrm{H}, J\left(\mathrm{CH}_{3}, \mathrm{CH}_{2}\right)=7.1\left(\mathrm{CH}_{3} \mathrm{CH}_{2} \mathrm{O}\right) ; 1.82 \mathrm{~d}, 3 \mathrm{H}$, $J\left(\mathrm{CH}_{3}, \mathrm{CH}\right)=1.2\left(\mathrm{CH}_{3}\right) ; 4.13 \mathrm{q}, 2 \mathrm{H}, J\left(\mathrm{CH}_{2}, \mathrm{CH}_{3}\right)=7.1\left(\mathrm{OCH}_{2} \mathrm{CH}_{3}\right) ; 7.53 \mathrm{q}, 1 \mathrm{H}, J\left(\mathrm{CH}, \mathrm{CH}_{3}\right)$ $=1.2(\mathrm{CH}=) \cdot{ }^{13} \mathrm{C}$ NMR $\left(\mathrm{CDCl}_{3}, 125.7 \mathrm{MHz}\right): 8.79\left(\mathrm{CH}_{3}\right) ; 15.34\left(\mathrm{CH}_{3} \mathrm{CH}_{2} \mathrm{O}\right) ; 70.98$ $\left(\mathrm{OCH}_{2} \mathrm{CH}_{3}\right) ; 110.19(\mathrm{C}=) ; 130.47(\mathrm{NCO}) ; 162.82(\mathrm{CH}=) ; 165.52(\mathrm{CO})$.

\section{1-(2,4,6-Tri-tert-butylphenyl)-3-((E)-3-ethoxyacryloyl)urea (11a)}

${ }^{1} \mathrm{H}$ NMR $\left(\mathrm{CDCl}_{3}, 500.0 \mathrm{MHz}\right): 1.30 \mathrm{~s}, 9 \mathrm{H}\left(\left(\mathrm{CH}_{3}\right)_{3} \mathrm{C}^{-} 4^{\prime}\right) ; 1.32 \mathrm{t}, 3 \mathrm{H}, J\left(\mathrm{CH}_{3}, \mathrm{CH}_{2}\right)=7.1$ $\left(\mathrm{CH}_{3} \mathrm{CH}_{2} \mathrm{O}\right) ; 1.40 \mathrm{~s}, 18 \mathrm{H}\left(\left(\mathrm{CH}_{3}\right)_{3} \mathrm{C}^{-2} 2^{\prime}, 6^{\prime}\right) ; 3.90 \mathrm{q}, 2 \mathrm{H}, J\left(\mathrm{CH}_{2}, \mathrm{CH}_{3}\right)=7.1\left(\mathrm{OCH}_{2} \mathrm{CH}_{3}\right) ; 5.26$ $\mathrm{d}, 1 \mathrm{H}, J(5,6)=12.2(\mathrm{H}-5) ; 7.40 \mathrm{~s}, 2 \mathrm{H},\left(\mathrm{H}^{\prime} 3^{\prime}, 5^{\prime}\right) ; 7.72 \mathrm{~d}, 1 \mathrm{H}, J(6,5)=12.2(\mathrm{H}-6) ; 8.54 \mathrm{bs}, 1$ $\mathrm{H}(\mathrm{H}-3) ; 10.29 \mathrm{bs}, 1 \mathrm{H}(\mathrm{H}-1) .{ }^{13} \mathrm{C}$ NMR $\left(\mathrm{CDCl}_{3}, 125.7 \mathrm{MHz}\right): 14.48\left(\mathrm{CH}_{3} \mathrm{CH}_{2} \mathrm{O}\right) ; 31.38$ $\left(\left(\mathbf{C H}_{3}\right)_{3} \mathrm{C}^{-} 4^{\prime}\right) ; 31.86\left(\left(\mathrm{CH}_{3}\right)_{3} \mathrm{C}-2^{\prime}, 6^{\prime}\right) ; 34.97 \quad\left(\left(\mathrm{CH}_{3}\right)_{3} \mathbf{C}^{\prime}-4^{\prime}\right) ; 36.19 \quad\left(\left(\mathrm{CH}_{3}\right)_{3} \mathbf{C}^{\prime} 2^{\prime}, 6^{\prime}\right) ; 67.87$ $\left(\mathrm{OCH}_{2} \mathrm{CH}_{3}\right) ; 97.48$ (C-5); $122.93\left(\mathrm{C}-3^{\prime}, 5^{\prime}\right) ; 129.26$ (C-1'); 148.01 (C-2’,6’); 149.55 (C-4'); 154.77 (C-2); 163.84 (C-6); 168.26 (C-4).

HR-MS for $\mathrm{C}_{24} \mathrm{H}_{39} \mathrm{~N}_{2} \mathrm{O}_{3}(\mathrm{M}+\mathrm{H})^{+}$calcd 403.2955, found 403.2955.

\section{1-(2,4,6-Tri-tert-butylphenyl)-3-((E)-3-ethoxy-2-methylacryloyl)urea (11b)}

${ }^{1} \mathrm{H}$ NMR $\left(\mathrm{CDCl}_{3}, 500.0 \mathrm{MHz}\right): 1.31 \mathrm{~s}, 9 \mathrm{H}\left(\left(\mathrm{CH}_{3}\right)_{3} \mathrm{C}_{-4} 4^{\prime}\right) ; 1.33 \mathrm{t}, 3 \mathrm{H}, J\left(\mathrm{CH}_{3}, \mathrm{CH}_{2}\right)=7.1$ $\left(\mathrm{CH}_{3} \mathrm{CH}_{2} \mathrm{O}\right) ; 1.42 \mathrm{~s}, 18 \mathrm{H}\left(\left(\mathrm{CH}_{3}\right)_{3} \mathrm{C}-2^{\prime}, 6^{\prime}\right) ; 1.84 \mathrm{~d}, 3 \mathrm{H}, J\left(\mathrm{CH}_{3}, 6\right)=1.2\left(5-\mathrm{CH}_{3}\right) ; 4.04 \mathrm{q}, 2 \mathrm{H}$, $J\left(\mathrm{CH}_{2}, \mathrm{CH}_{3}\right)=7.1\left(\mathrm{OCH}_{2} \mathrm{CH}_{3}\right) ; 7.43 \mathrm{~s}, 2 \mathrm{H},\left(\mathrm{H}^{\prime} 3^{\prime}, 5^{\prime}\right) ; 7.58 \mathrm{q}, 1 \mathrm{H}, J\left(6, \mathrm{CH}_{3}\right)=1.2(\mathrm{H}-6) ; 7.95$ bs, $1 \mathrm{H}(\mathrm{H}-3) ; 10.45$ bs, $1 \mathrm{H}(\mathrm{H}-1) .{ }^{13} \mathrm{C} \mathrm{NMR}\left(\mathrm{CDCl}_{3}, 125.7 \mathrm{MHz}\right): 8.83\left(5-\mathrm{CH}_{3}\right) ; 15.44$ $\left.\left(\mathrm{CH}_{3} \mathrm{CH}_{2} \mathrm{O}\right) ; 31.37\left(\left(\mathrm{CH}_{3}\right)_{3} \mathrm{C}^{\prime} 4^{\prime}\right) ; 31.87\left(\left(\mathrm{CH}_{3}\right)_{3} \mathrm{C}-2^{\prime}\right)^{\prime}\right) ; 34.98\left(\left(\mathrm{CH}_{3}\right)_{3} \mathrm{C}^{\prime}-4^{\prime}\right) ; 36.20\left(\left(\mathrm{CH}_{3}\right)_{3} \mathrm{C}-\right.$ 2',6'); $70.54\left(\mathrm{OCH}_{2} \mathrm{CH}_{3}\right) ; 105.68$ (C-5); $123.04\left(\mathrm{C}-3^{\prime}, 5^{\prime}\right) ; 129.30$ (C-1'); 147.97 (C-2',6' $)$; 149.53 (C-4'); 154.33 (C-2); 158.71 (C-6); 169.64 (C-4).

HR-MS for $\mathrm{C}_{25} \mathrm{H}_{41} \mathrm{~N}_{2} \mathrm{O}_{3}(\mathrm{M}+\mathrm{H})^{+}$calcd 417.3112, found 417.3111.

\section{Methyl (E)-3-ethoxy-2-methylacryloylcarbamate (12)}

${ }^{1} \mathrm{H}$ NMR $\left(\mathrm{CDCl}_{3}, 500.0 \mathrm{MHz}\right): 1.33 \mathrm{t}, 3 \mathrm{H}, J\left(\mathrm{CH}_{3}, \mathrm{CH}_{2}\right)=7.1\left(\mathrm{CH}_{3} \mathrm{CH}_{2} \mathrm{O}\right) ; 1.83 \mathrm{~d}, 3 \mathrm{H}$, $J\left(\mathrm{CH}_{3}, 6\right)=1.2\left(5-\mathrm{CH}_{3}\right) ; 3.79 \mathrm{~s}, 3 \mathrm{H}\left(\mathrm{CH}_{3} \mathrm{O}\right) ; 4.08 \mathrm{q}, 2 \mathrm{H}, J\left(\mathrm{CH}_{2}, \mathrm{CH}_{3}\right)=7.1\left(\mathrm{OCH}_{2} \mathrm{CH}_{3}\right) ; 7.42$

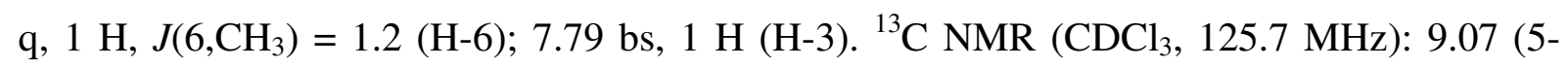


$\left.\mathrm{CH}_{3}\right) ; 15.26\left(\mathrm{CH}_{3} \mathrm{CH}_{2} \mathrm{O}\right) ; 52.72\left(\mathrm{CH}_{3} \mathrm{O}\right) ; 70.12\left(\mathrm{OCH}_{2} \mathrm{CH}_{3}\right) ; 107.09$ (C-5); 151.93 (C-2); 157.59 (C-6); 165.97 (C-4).

HR-MS for $\mathrm{C}_{8} \mathrm{H}_{13} \mathrm{NO}_{4} \mathrm{Na}(\mathrm{M}+\mathrm{H}+\mathrm{Na})^{+}$calcd 210.0742, found 210.0741 .

Supporting Information (see footnote on the first page of this article): Kinetics measurements and computational data including .

\section{Acknowledgments}

We gratefully acknowledge the financial support by the Ministry of Education, Youth, and Sports of the Czech Republic (Research projects Z40550506, 2B06065, and LC512) and the Ministry of Health (Grant NR/9138 - 3).

[1] Galmarini C. M., Mackey J. R.,Dumontet C. The Lancet Oncology 2002, 3( 7), 415 424.

[2] Miura S, Izuta S. Curr. Drug. Targets. 2004, 5(2), 191-195.

[3] Simons C., Wu Q., Htar T.T. Curr. Top. Med. Chem. 2005, (13), 1191-1203.

[4] Schneller, S.W. Curr. Top. Med. Chem. 2002, 2(10), 1087-1092.

[5] Jeong L.S., Lee J.A. Antivir. Chem. Chemother. 2004, 15(5), 235-250.

[6] Holý A. Antiviral Res. 2006, 71(2-3), 248-253.

[7] Rejman D., Masojídková M., De Clercq E., Rosenberg I.: Nucleosides \& Nucleotides 2001, 20 (8) 1497-1522.

[8] Kočalka, P.; Pohl, R.; Rejman, D.; Rosenberg, I. Tetrahedron 2006, 62, 5763-5774

[9] Rejman D., Kočalka P., Buděšínský M., Pohl R., Rosenberg I.: Tetrahedron 2007, 63, $5,1243-1253$.

[10] Yamada K., Sakata S., Yoshimura Y. J. Org. Chem. 1998, 63, 6891-6899.

[11] Lu W., Sengupta S., Petersen J. L., Akhmedov N. G., Shi X. J. Org. Chem. 2007, 72, 5012-5015.

[12] Michel B. Y., Strazewski P. Tetrahedron 2007, 63, 9836-9841.

[13] Abrams, H. M.; Ho, L.; Chu, S. H. Journal of Heterocyclic Chemistry 1981, 18, 947 951.

[14] Maruyama, Tokumi; Kozai, Shigetada; Uchida, Mayuko Nucleosides \& Nucleotides 1999, 18(4-5), $661-672$.

[15] Takenaka, Keiko; Muraoka, Masako; Tsuji, Tadakazu Journal of Heterocyclic Chemistry 1997, 34(2), 669 - 673. 
[16] Shaw G., Warrener R. N. J. Chem. Soc.1958, 153-156. Shaw G., Warrener R. N. J. Chem. Soc. 1958, 157-161.

[17] Shealy Y. F., O’Dell C. A.: J. Heterocyclic Chem. 1976, 13, 1015-1020.

[18] Wyatt P. G., Anslow A. S., Coomber B. A., Cousins R. P. C., Evans D. N., Gilbert V. S., Humber D. C., Paternoster I. L., Sollis S. L., Tapolczay D. J., Weingarten G. G.: Nucleosides Nucleotides 1995, 14, 2039-2049.

[19] Hřebabecký H., Masojídková M., Holý A.: Collect. Czech. Chem. Commun. 2005, 70, 519-538.

[20] Csuk R., von Scholz Y.: Tetrahedron 1995, 51, 7193-7206.

[21] Borthwick A. D., Evans D. N., Kirk B. E., Biggadike K., Exal A. M., Youds P., Roberts S. M., Knight D. J., Coates A. V.: J. Med. Chem. 1990, 33, 179-186.

[22] Bodenteich M., Marquez V. E., Barchi J. J., Hallows W. H., Goldstein B. M., Driscoll J. S.: J. Org. Chem. 1993, 58, 6009-6015.

[23] Hosono F., Nishiyama S., Yamamura S.: Tetrahedron, 1994, 50, 47, 13335-13346.

[24] Raganathan S., George K. S.: Tetrahedron 1997, 53, 9, 3347-3362.

[25] Miyabe H., Kanehira S., Kume K., Kandori H., Naito T.: Tetrahedron, 1998, 54, 5883-5892.

[26] Moon H. R., Kim H. O., Chun M. W., Jeong L. S.: J. Org. Chem.. 1999, 64, 47334741.

[27] Wang P., Gullen B., Newton M. G., Cheng Y. C., Shinazi R. F., Chu C.K.: J. Med. Chem. 1999, 42, 3390-3399.

[28] Bera S., Mickle T., Nair V.: Nucleosides Nucleotides 1999, 18 (11\&12), 2379-2395.

[29] Kim H. S., Ravi R. G., Marquez V. E., Maddileti S., Wihlborg A. K., Erlinge D., Malmsjö M., Boyer J. L., Harden T. K., Jacobson K. A.: J. Med. Chem. 2002, 45, 208-218.

[30] Nieto M. I., Caamaño O., Fernández F., Gómez M., Balzarini J., DeClercq E.: Nucleosides Nucleotides 2002, 21 (3), 243-255.

[31] Kim S. A., Lee H. M., Ryu J. S., Kim H. S.: Synlett 2007, 7, 1055-1058.

[32] Migliore M. D., Zonta N., McGuigan C., Henson G., Andrei G., Snoeck R., Balzarini J.: J. Med. Chem. 2007, 50, 6485-6492.

[33] Shealy Y. F., O’Dell C. A.: J. Heterocyclic Chem. 1976, 13, 1041-1047.

[34] Rejman D., Kovačková S., Pohl R., Dračínský M., Fiedler P., Rosenberg I. Tetrahedron, 2009, 65, 41, 8513-8523.

[35] Harvey, J. N. Faraday Discuss. 2009, 145, 487-505. 
[36] R. Ahlrichs, M. Bär, M. Häser, H. Horn, C. Kölmel, Chem. Phys. Lett. 1989 162, 165169.

[37] J. P. Perdew, K. Burke, M. Ernzerhof, Phys. Rev. Lett., 1996, 77, 3865-3868.

[38] (a) A. D. Becke, Phys. Rev. A 1988, 38, 3098-3100. (b) C. T. Lee, W. T. Yang, R. G. Parr, Phys. Rev. B 1988, 37, 785-789. (c) A. D. Becke, J. Chem. Phys. 1993, 98, 5648-5652. (d) P. J. Stephens, F. J. Devlin, C. F. Chabalowski, M. J. Frisch, J. Phys. Chem. 1994, 98, 11623-11627.

[39] K. Eichkorn, O. Treutler, H. Öhm, M. Häser, R. Ahlrichs, Chem. Phys. Lett. 1995, 240, 283-290.

[40] K. Eichkorn, F. Weigen, O. Treutler, R. Ahlrichs, Theor. Chim. Acta 1997, 97, 119124.

[41] (a) A. Schäfer, H. Horn, R. Ahlrichs, J. Chem. Phys. 1992, 97, 2571-2577. (b) F. Weigend, R. Ahlrichs, Phys. Chem. Chem. Phys. 2006, 7, 3297-3305.

[42] A. Klamt, G. Schuurmann, J. Chem. Soc.-Perkin Trans. 2 1993, 799-805.

[42] A. Schäfer, A. Klamt, D. Sattel, J. C. W. Lohrenz, F. Eckert, Phys. Chem. Chem. Phys. 2000, 2, 2187-2193.

[43] Grimme, S. J Comput Chem 2004, 25, 1463-1473.

[44] Jensen, F. Introduction to Computational Chemistry; John Wiley \& Sons: New York, 1999. 
Table 1 . The kinetics of $\mathbf{7 a - d}$ degradation monitored by ${ }^{1} \mathrm{H}$ NMR.

[a] $k_{+}=1.83 k_{-}$

\begin{tabular}{lllll}
\hline Degradation & Reaction & Kinetics & $k\left(\mathrm{hr}^{-1}\right)$ & $t_{1 / 2}(\mathrm{hr})$ \\
\hline $\mathbf{7 a}$ & reversible & first-order & $3.00 \times 10^{-2[a]}$ & 23.1 \\
$\mathbf{7 b}$ & irreversible & first-order & 2.32 & 0.30 \\
$\mathbf{7 c}$ & no degradation observed & & \\
$\mathbf{7 d}$ & no degradation observed & & \\
\hline
\end{tabular}


Table 2. The theoretical calculations of reaction free energies and activation barriers carried out using the B3LYP/def2-TZVP//RI-PBE/def2-SVP method. The solvation effects were included through COSMO calculations and the energies corrected for zero-point energies, thermal corrections to enthalpy and entropic terms (using ideal gas approximation) to obtain free energy estimates. The experimental data are shown in the right part of the table $\left(\Delta G_{\text {exp }}^{\ddagger}\right.$ were calculated using Eyring equation: $\left.\Delta G^{\ddagger}=R T[23.76-\ln (k / T)]\right)$.

\begin{tabular}{lllll}
\hline Degradation & $\begin{array}{l}\Delta G_{\mathrm{calc}} \\
\left(\mathrm{kJ} \cdot \mathrm{mol}^{-1}\right)\end{array}$ & $\begin{array}{l}\Delta G_{\mathrm{calc}}^{\ddagger} \\
\left(\mathrm{kJ}_{\mathrm{mol}}{ }^{-1}\right)\end{array}$ & $\begin{array}{l}k_{\exp } \\
\left(\mathrm{s}^{-1}\right)\end{array}$ & $\begin{array}{l}\Delta G_{\text {exp }}^{\ddagger} \\
\left(\mathrm{kJ} \cdot \mathrm{mol}^{-1}\right)\end{array}$ \\
\hline $\mathbf{7 a}$ & -24.6 & 84.1 & $8.33 \times 10^{-6}$ & 102.0 \\
$\mathbf{7 b}$ & -30.2 & 72.0 & $6.44 \times 10^{-4}$ & 91.2 \\
$\mathbf{7 c}$ & -3.9 & 88.4 & - & - \\
$\mathbf{7 d}$ & -15.1 & 79.1 & - & - \\
\hline
\end{tabular}


Table 3. The kinetics of the reaction of the reagents 7a-d with 2,4,6-tri-tert-butylaniline in dry $\mathrm{CDCl}_{3}$ at $25^{\circ} \mathrm{C}$.

\begin{tabular}{lll}
\hline Reagent & $k\left(\mathrm{hr}^{-1}\right)$ & $t_{1 / 2}(\mathrm{hr})$ \\
\hline $\mathbf{7 a}$ & $2.7 \times 10^{-2}$ & 25.7 \\
$7 \mathbf{b}$ & 1.2 & 0.6 \\
$\mathbf{7 c}$ & $7.4 \times 10^{-2}$ & 9.4 \\
$\mathbf{7 d}$ & $2.7 \times 10^{-1}$ & 2.6 \\
\hline
\end{tabular}

